\title{
Design of Novel Thermal Barrier Coatings with Reduced Thermal Conduction and Thermal Radiation
}

\author{
By \\ Dongmei Wang
}

\begin{abstract}
A thesis submitted to the
Faculty of Graduate Studies and Research

in partial fulfillment of the requirements for

The Degree of Doctor of Philosophy
\end{abstract}
Ottawa-Carleton Institute for Mechanical and Aerospace Engineering
Department of Mechanical and Aerospace Engineering
Carleton University
Ottawa, Ontario, Canada

Nov. 2006

(C) Dongmei Wang, 2006 


$\begin{array}{ll}\begin{array}{l}\text { Library and } \\ \text { Archives Canada }\end{array} & \begin{array}{l}\text { Bibliothèque et } \\ \text { Archives Canada }\end{array} \\ \begin{array}{l}\text { Published Heritage } \\ \text { Branch }\end{array} & \begin{array}{l}\text { Direction du } \\ \text { Patrimoine de l'édition }\end{array} \\ \begin{array}{l}\text { 395 Wellington Street } \\ \text { Ottawa ON K1A ON4 }\end{array} & \begin{array}{l}\text { 395, rue Wellington } \\ \text { Ottawa ON K1A ON4 } \\ \text { Canada }\end{array}\end{array}$

Your file Votre référence ISBN: 978-0-494-27116-2 Our file Notre référence ISBN: 978-0-494-27116-2

NOTICE:

The author has granted a nonexclusive license allowing Library and Archives Canada to reproduce, publish, archive, preserve, conserve, communicate to the public by telecommunication or on the Internet, loan, distribute and sell theses worldwide, for commercial or noncommercial purposes, in microform, paper, electronic and/or any other formats.

The author retains copyright ownership and moral rights in this thesis. Neither the thesis nor substantial extracts from it may be printed or otherwise reproduced without the author's permission.
AVIS:

L'auteur a accordé une licence non exclusive permettant à la Bibliothèque et Archives Canada de reproduire, publier, archiver, sauvegarder, conserver, transmettre au public par télécommunication ou par l'Internet, prêter, distribuer et vendre des thèses partout dans le monde, à des fins commerciales ou autres, sur support microforme, papier, électronique et/ou autres formats.

L'auteur conserve la propriété du droit d'auteur et des droits moraux qui protège cette thèse. $\mathrm{Ni}$ la thèse ni des extraits substantiels de celle-ci ne doivent être imprimés ou autrement reproduits sans son autorisation.
In compliance with the Canadian

Privacy Act some supporting forms may have been removed from this thesis.

While these forms may be included in the document page count, their removal does not represent any loss of content from the thesis.
Conformément à la loi canadienne sur la protection de la vie privée, quelques formulaires secondaires ont été enlevés de cette thèse.

Bien que ces formulaires aient inclus dans la pagination, il n'y aura aucun contenu manquant.

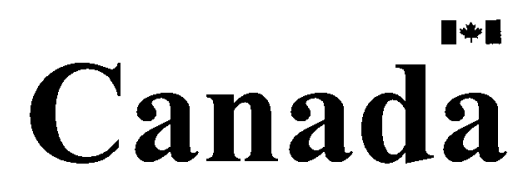




\section{Abstract}

The continual increase in gas turbine temperatures necessitates an advanced TBC system with the ability to reduce both thermal conduction and thermal radiation through the coatings. New TBC materials and structures are required in order to simultaneously lower the thermal conduction and thermal radiation to the metal substrate. To achieve these objectives, thermal conductivities of various metal oxides doped yttria stabilized zirconia bulk materials were studied by systematically doping metal oxides to 7YSZ material in order to find the optimal doping material. Additionally, based on the experimental results a model to predict thermal conductivity of the doped zirconia based ceramics was established, incorporating integrated defect scattering cluster consisted of substitutional atoms and oxygen vacancies. The calculated thermal conductivity of doped material obtained using this model was found to be very consistent with the experimental results. Furthermore, a novel multiple layered coating structure was designed with more than $80 \%$ hemispherical reflectance to radiation within a wavelength range of $0.3 \sim 5 \mu \mathrm{m}$. The structure consists of sets of high reflectance multiple layered stacks and a single layer ceramic material with low thermal conductivity. The simulated temperature distribution results showed that the this new structure, containing high reflectance multiple layered stacks, can achieve as much as $46^{\circ} \mathrm{C}$ temperature reduction on the metal surface when compared to a monolayered coating structure of the same thickness. The outcome of this study provides directions to future coating composition and microstructure design in combining the doped zirconia possessing the lowest thermal conductivity with the multiple layered coating structures. 


\section{Acknowledgements}

I would first like to express my sincere gratitude to my thesis supervisor, Dr. Xiao Huang, for her continued guidance and inspiration through the course of my studies. She has constantly supported me and imparted her wisdom to me not only in my research endeavors, but also towards all aspects of my life. She has been a mentor to me in the true sense of the word.

I would also like to express my gratitude to my co-supervisor, Dr. Prakash Patnaik, for providing me financial support and all the convenience of using NRC's facilities such that my research could be finished smoothly.

I would also like to thank Dr. Weijie Chen and Dr. Qi Yang for helping me on the measurement of the physical and mechanical properties; and also many thanks to Dr. Christian.Moreau and Mario.Lamontagne in IMI, NRC for providing the facility to measure thermal diffusivities of the samples. Without your help, I could not have written my thesis. And I sincerely appreciate your help. I would like to extend my appreciation to Dave Morphy, Ryan MacNeil, Robert Mckellar, David Chow and Olga Lupandina for your warmly technical support. Many thanks to the Department of Mechanical and Aerospace Engineering for the financial assistance awarded to me, which ensured me dedicate to my project. I would also like to express my gratitude to Fred Barrett for introducing me into the material world and starting to love the job as a materials lab TA.

Finally, I want to thank my family. Thank you for your patient and understanding. 


\section{Table of Contents}

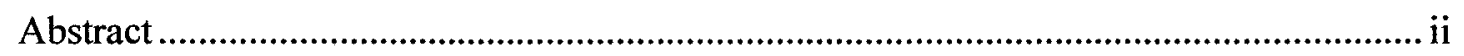

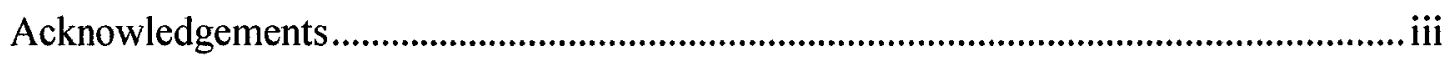

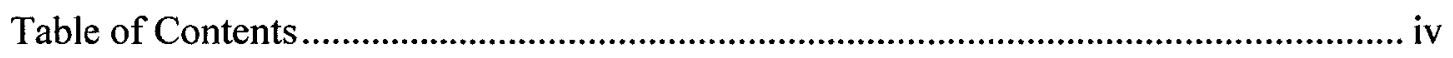

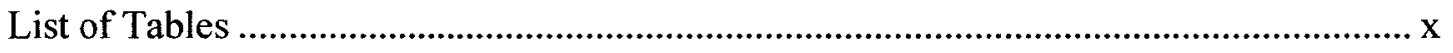

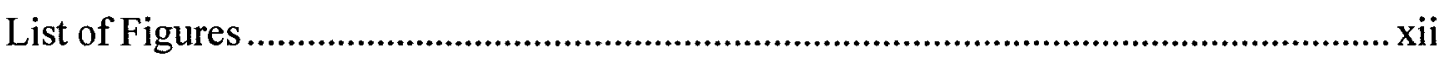

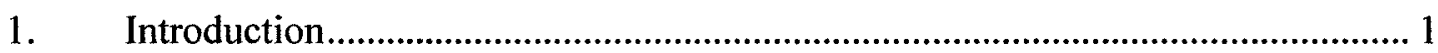

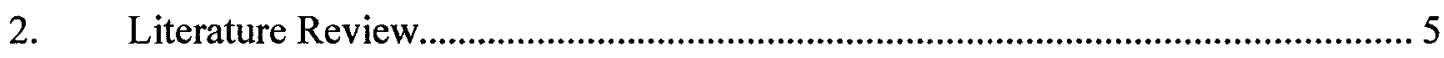

2.1. Thermal Barrier Coating Systems............................................................ 5

2.2. The Evolution of Thermal Barrier Coatings Systems..................................... 9

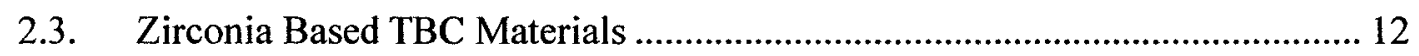

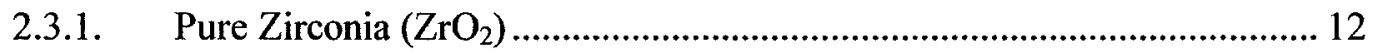

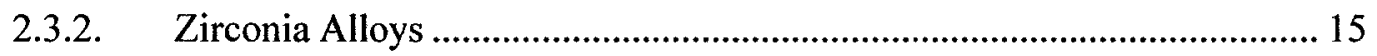

2.3.2.1. Mechanisms of phase stabilization ............................................... 15

2.3.2.2. Phase diagram of the yttria - zirconia system ................................ 17

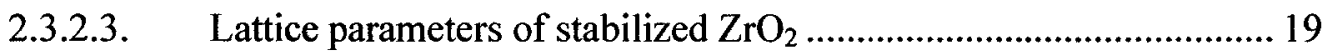

2.3.3. Microstructures and Properties of Tetragonal $\mathrm{ZrO}_{2}\left(t^{\prime}\right.$ phase $)$................. 21

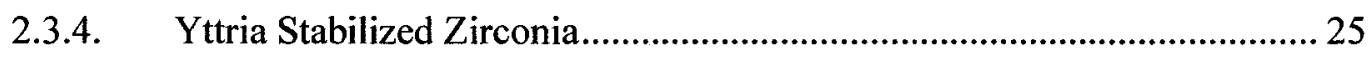

2.4. Thermal Barrier Coating Deposition Technologies ......................................... 27

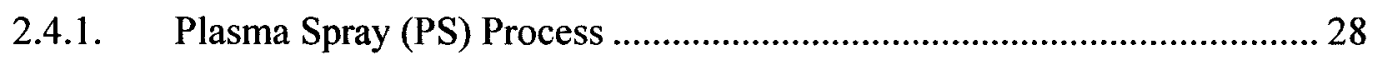

2.4.2. Coating Microstructures Produced by Plasma Spraying Deposition........ 29

2.4.3. Electron Beam Physical Vapor Deposition (EB-PVD) Process ................ 31 iv 
2.4.4. Coating Microstructure Produced by EB-PVD Process ........................... 33

2.4.5. Comparison of Coatings Produced by Plasma Spraying and EB-PVD .... 34

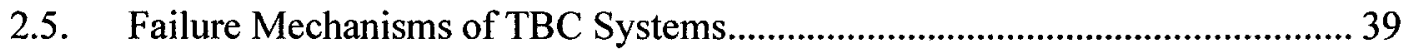

2.5.1. Failure Mechanisms of Thermal Barrier Coatings................................. 40

2.5.1.1. Failure mode and mechanisms of plasma sprayed TBCs ................ 41

2.5.1.2. Failure mode of EB-PVD coatings ............................................... 43

2.5.2. Hot Corrosion Failure ..................................................................... 45

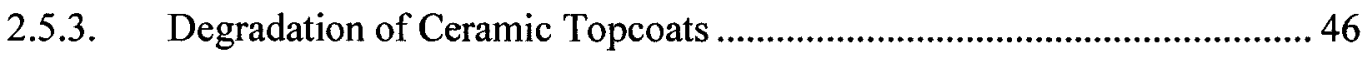

2.5.3.1. Sintering and densification of ceramic coatings ......................... 47

2.5.3.2. Aging and phase decomposition ................................................ 49

2.6. Heat Transfer through Ceramic Materials ............................................... 50

2.6.1. Thermal Conduction in Ceramic Materials........................................... 51

2.6.1.1. Intrinsic thermal conductivity at high temperatures ...................... 55

2.6.1.2. Phonon scattering by static imperfections ......................................5 59

2.6.1.3. Klemens-Callaway model of thermal conductivity .......................... 62

2.6.2. Radiation Transport through Zirconia Based Ceramic Coatings..............6 65

2.6.2.1. Radiation properties of zirconia based ceramics ............................6 65

2.6.2.2. Radiation transfer within ceramic coating materials ......................69 69

2.7. Methods to Reduce Thermal Conduction through TBCS.............................. 73

2.7.1. Introduction of Crystal Defects........................................................ 73

2.7.2. Modification of Thermal Barrier Coating Microstructures ..................... 78

2.7.3. Development of New Generation of TBC Materials ...........................82

2.8. Methods to Reduce Thermal Radiation through TBCS .................................. 87 


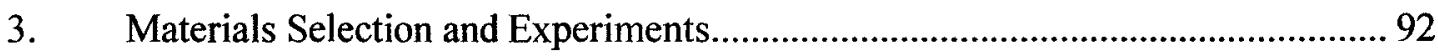

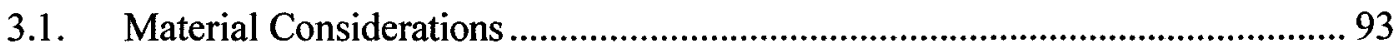

3.2. Fabrication of Co-doped Zirconia-based Bulk Materials ................................ 95

3.2.1. Experimental Considerations ........................................................... 95

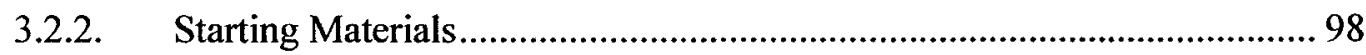

3.2.3. Powder Blending and Comminuting............................................... 100

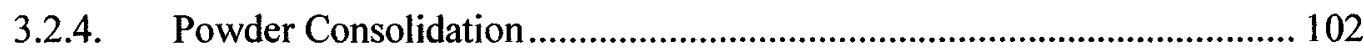

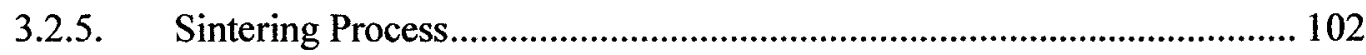

4. Characterization of the Alloyed Powders and the Sintered Materials ................ 105

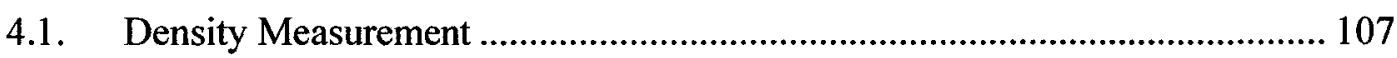

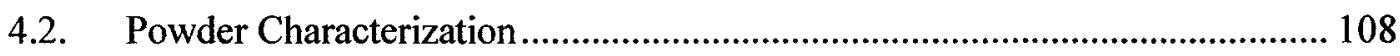

4.3. Microstructural Examination of the Sintered Samples ............................... 110

4.4. Volume Fraction of Porosity Inside the Sintered Samples ............................ 111

4.5. Elemental Composition Analysis.......................................................... 112

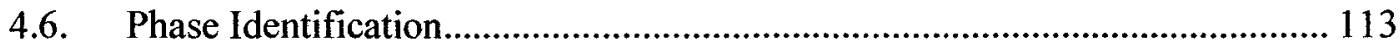

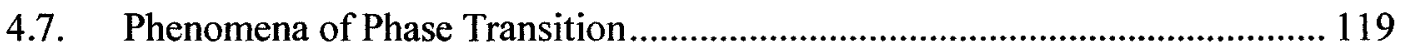

4.7.1. Basic Principle and Method of DSC ..................................................... 119

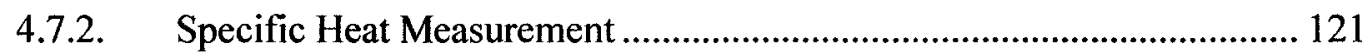

4.8. Mechanical Properties of the Sintered Samples.......................................... 123

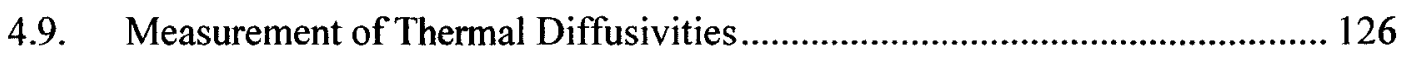

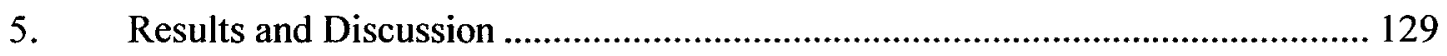

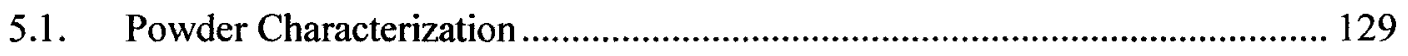

5.1.1. SEM Images of All As-received Powders .......................................... 129 
5.1.2. SEM Images of Selected Powders after Mechanical Alloying ............... 132

5.2. Microstructures of the Sintered Samples .................................................. 135

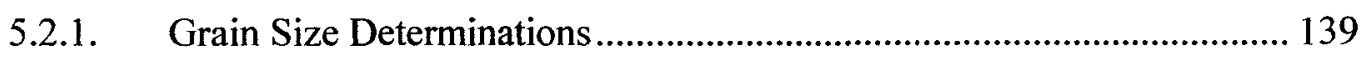

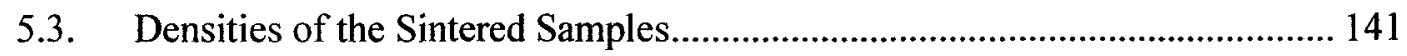

5.4. Porosities Within the Sintered Samples.................................................... 143

5.5. Elemental Composition Analysis........................................................... 145

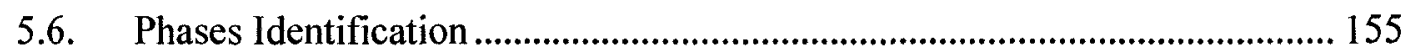

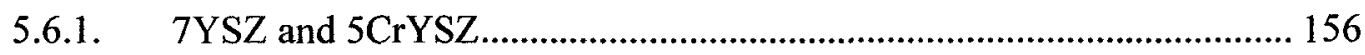

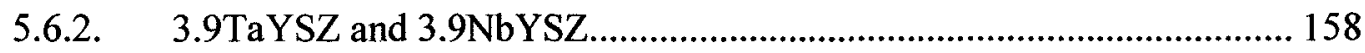

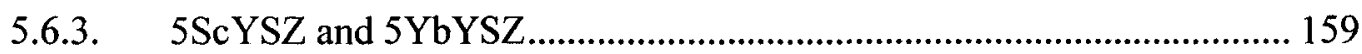

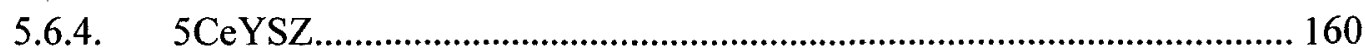

5.7. Prediction of Phases from Reference Phase Diagrams ................................ 162

5.8. Estimation of Compositions of Dopants and Oxygen Vacancies .................. 167

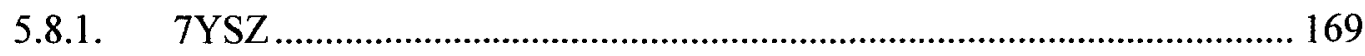

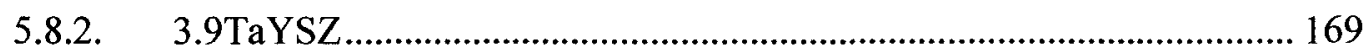

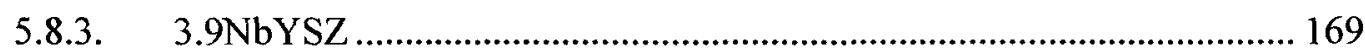

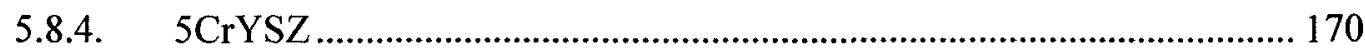

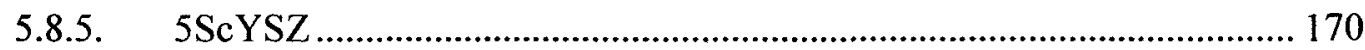

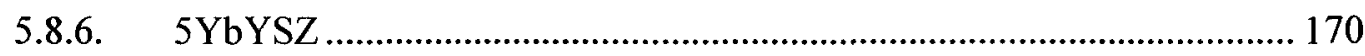

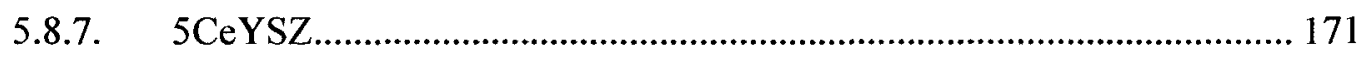

5.9. Effects of Dopants on Phase Transformations and Specific Heats................. 173

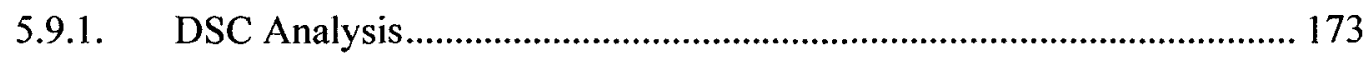

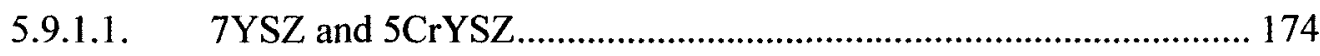

vii 
5.9.1.2. 3.9MYSZ $(\mathrm{M}=\mathrm{Ta}$ and $\mathrm{Nb})$

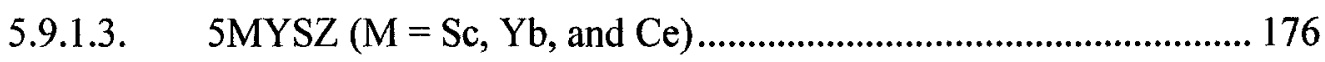

5.9.2. Specific Heat of Each Doped Material ..................................................... 178

5.10. Effects of Dopants on Materials Elastic Modulus ............................................ 181

5.11. Effects of Dopants on Thermal Diffusivity ………………………………........ 183

5.12. Calculation of Thermal Conductivity ……………………………………....... 185

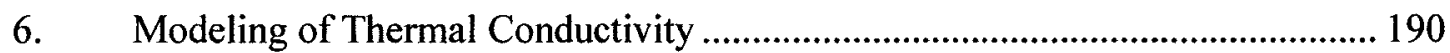

6.1. Thermal Resistance of Zirconia Based Materials ............................................... 191

6.2. Calculation of Thermal Conductivities of the Doped Zirconia Based Materials

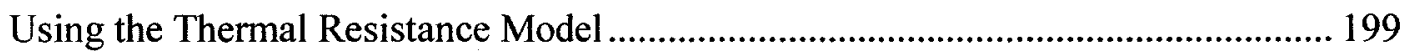

7. Multiple Layered Thermal Barrier Coatings Design ............................................ 202

7.1. Design of Multiple Layered Stacks with High Reflectance.............................. 202

7.1.1. Fundamental Concept of Multiple Beam Interference............................... 203

7.1.2. Design of Multiple Layered Stacks........................................................... 207

7.2. Multiple Layered Coating Structures Incorporating Multiple Layered Stacks 210

7.3. Radiation Transport through the Multiple Layered Coating Structures ..........213

8. Governing Equations of the Designed TBC Structures ........................................ 215

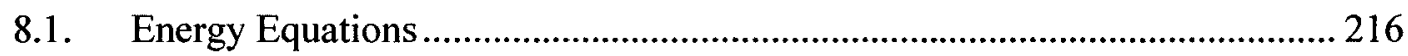

8.2. Evaluation of Radiation Flux for Structures A, B, C, and D .......................... 219

8.2.1. Radiation Flux within the Wavelength Range of $0.3 \sim 5 \mu \mathrm{m} \ldots \ldots \ldots \ldots \ldots \ldots \ldots . . .220$

8.2.2. Radiation Flux within the Wavelength Range of $5 \sim 10 \mu \mathrm{m} \ldots \ldots \ldots \ldots \ldots \ldots \ldots \ldots . . . . . . . . . .223$

8.3. Temperature Distributions through the Multi- Layered Coating Systems...... 227

8.3.1. Metal Surface Temperatures ................................................................... 229 viii 


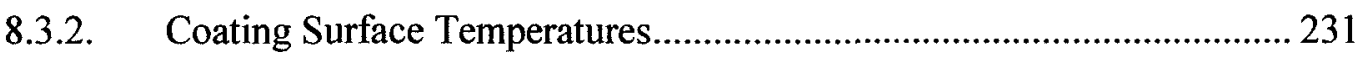

8.3.3. Optimum Multiple Layered Structures .............................................. 232

8.3.4. Novelty of the Current Coating Structures .......................................... 234

8.3.5. Feasibility of the Deposition of the Multiple Layered Coatings............. 235

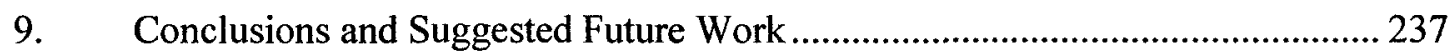

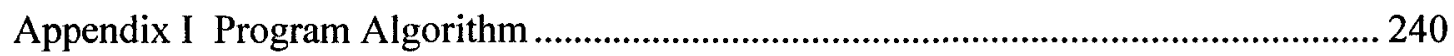

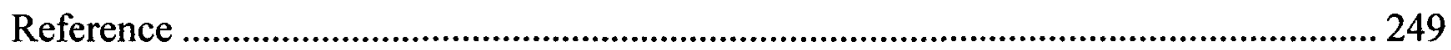




\section{List of Tables}

Table 1 Lattice parameters of pure zirconia for three phases [20] ............................... 15

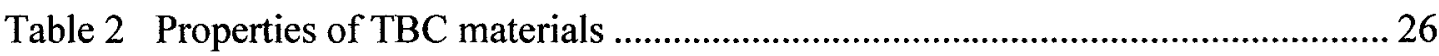

Table 3 Thermal expansion coefficients and Young's moduli of various zirconates..... 86

Table 4 Parameters of selected metal oxides used as additions to 7YSZ .......................93

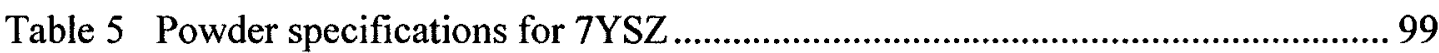

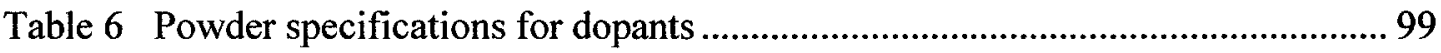

Table 7 Compositions of the dopant cations in doped 7YSZ systems........................ 100

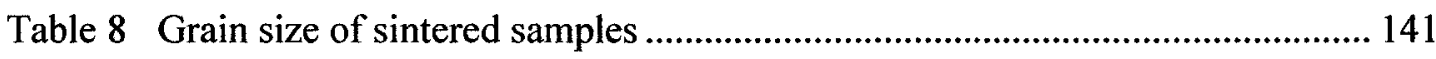

Table 9 Measured density, theoretical density and relative density .......................... 143

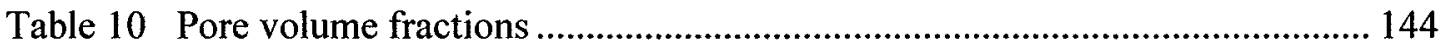

Table 11 Phases present and phase concentrations obtained from XRD patterns ........ 162

Table 12 The calculated oxygen percentages for each sample .................................... 164

Table 13 The relative content of each phase calculated for all the samples ................. 166

Table 14 The contents of dopants and oxygen vacancies within each phase and phase

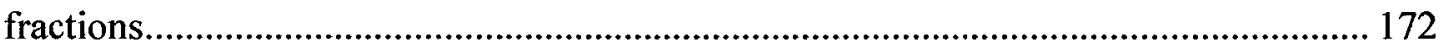

Table 15 Summary of phase transformation temperatures upon DSC heating............. 173

Table 16 The values of measured specific heat of doped materials using DSC .......... 180

Table 17 The values of specific heat of doped materials from publications ............... 180

Table 18 Values of elastic modulus and hardness of each doped material at $50 \mathrm{mN}$ loads 
Table 19 Thermal diffusivity $(\alpha)$ and its standard deviation of each doped material at

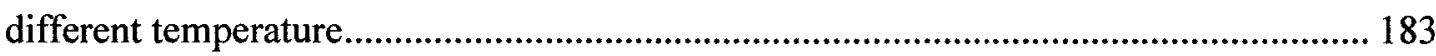

Table 20 The data for determining parameters $\varepsilon_{11}, \varepsilon_{12}, \varepsilon_{21}, \varepsilon_{22}$ and $W_{P c} \ldots \ldots \ldots \ldots \ldots . . . . . . .198$

Table 21 Data for calculation of thermal resistance using the established model ........ 200

Table 22 Comparison of thermal conductivities between the calculated and measured results 201

Table 23 Physical thickness of each layer for the first and last stacks in designing a multiple layered TBC coating system. 209

Table 24 Numerical Values and References

Table 25 Temperatures on coating and metal surfaces, total heat and radiation fluxes through structures A, B, C and D 


\section{List of Figures}

Figure 1 Increase in operating temperature of turbine components as a result of new superalloys, advanced manufacturing technology and thermal barrier coating development [1]

Figure 2 Typical Thermal Barrier Coating System......................................................... 5

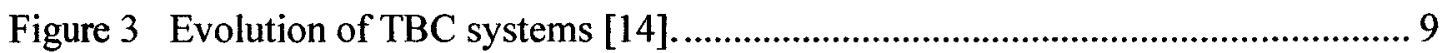

Figure 4 Fluorite structure showing the eightfold coordination of the cubic $\mathrm{ZrO}_{2}$ [16]. 13

Figure 5 Crystal structure of two face-centered tetragonal zirconia [16] ..................... 13

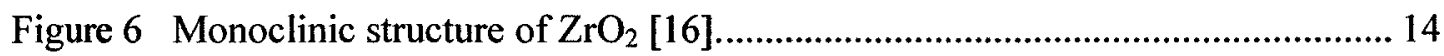

Figure 7 The pseudo-binary $\mathrm{YO}_{1.5}-\mathrm{ZrO}_{2}$ phase diagram. The labeling across the bottom of the diagram shows metastable phase fields upon rapid cooling [25]

Figure 8 Lattice parameters for the cubic, tetragonal and monoclinic phases as a

function of $\mathrm{mol} \% \mathrm{YO}_{1.5}[20]$

Figure 9 Microstructures of $\mathrm{ZrO}_{2}-4 \mathrm{~mol}_{2} \mathrm{Y}_{2} \mathrm{O}_{3}$ prepared by arc-melting; (a) bright field image and (b) dark field image taken with a (112) reflection [17].

Figure 10 The microstructure in $\mathrm{ZrO} 2-4 \mathrm{~mol} \%$ (a) $\mathrm{Nd}_{2} \mathrm{O}_{3}$, (b) $\mathrm{Sm}_{2} \mathrm{O}_{3}$, (c) $\mathrm{Gd}_{2} \mathrm{O}_{3}$, (d)

$\mathrm{Y}_{2} \mathrm{O}_{3}$, (e) $\mathrm{Er}_{2} \mathrm{O}_{3}$ and (f) $\mathrm{Yb}_{2} \mathrm{O}_{3}$ prepared by arc-melting [17]

Figure 11 Phase stability of doped binary $\mathrm{ZrO}_{2}-\mathrm{MO}_{1.5}$ compositions (circles) and doped ternary $\mathrm{ZrO}_{2}-\left(\mathrm{Y}_{0.5} \mathrm{M}_{0.5}\right) \mathrm{O}_{1.5}$ compositions (diamonds) [30].

Figure 12 Effects of the yttria content on the lifetime of a $\mathrm{ZrO}_{2}-\mathrm{Y}_{2} \mathrm{O}_{3} / \mathrm{Ni}-16 \mathrm{Cr}-6 \mathrm{Al}-$ $0.2 \mathrm{Y}$ thermal barrier coatings withstood 15001 hour cycles between $990^{\circ} \mathrm{C}-280^{\circ} \mathrm{C}$ [34]. 
Figure 13 Schematic of the plasma spray process.

Figure 14 Photomicrograph of an APS TBC showing a laminar structure [37]............ 30

Figure 15 Schematic diagram of the principle of EB-PVD process. ............................ 31

Figure 16 Schematic diagram of EB-PVD unit with 6 EB guns and 3 continuous ingots feeding systems $[38,39]$. 32

Figure 17 Photomicrograph of a thermal barrier coating (TBC) obtained by electron beam physical vapor deposition (EB-PVD) [38].

Figure 18 The comparison of thermal cyclic lives of air plasma sprayed TBCs and EBPVD TBCs [41] 36

Figure 19 Young's moduli of solid sintered 8wt\% PYSZ, plasma sprayed and EB-PVD

$8 \mathrm{wt} \%$ PYSZ TBC systems measured by dynamic techniques [42]. 36

Figure 20 SEM image of a cross section through an as-deposited material revealing the $\mathrm{TBC}$, the TGO and the bond coat. The arrows highlight imperfections around the TBC/TGO interface [57]. 41

Figure 21 SEM images of delaminations introduced by an as -deposited plasma sprayed coating [56]. 42

Figure 22 Schematic illustrations of the failure mechanisms for Plasma Sprayed TBCs $[57]$

Figure 23 Schematic indicating the sequence of events when a compressed film buckles and spalls away from a substrate $[53,54]$. 44

Figure 24 Interface separations for the EB-PVD TBCs after 180 thermal cycles [57]. . 45 xiii 
Figure 25 Cross-sectional images showing the microstructural evolution of EB-PVD coating with different temperatures and times (a) as-deposited and (b) after heat treatment at $1200^{\circ} \mathrm{C}$ for 120 hours [63]. 47

Figure 26 Young' modulus of EB-PVD coating increases with time at $1200^{\circ} \mathrm{C}[63] \ldots 48$ Figure 27 Micro-thermal stress and density as a function of distance from the interface to top surface of free-standing EB-PVD coating with different times [63]. 49

Figure 28 (a). Normal process with $\bar{k}_{1}+\bar{k}_{2}$ within the first Brillouin zone. (b). Umklapp process with $\bar{k}_{1}+\bar{k}_{2}$ lying outside the first Brillouin zone and being brought back by a reciprocal lattice vector.

Figure 29 Room-temperature (a) hemispherical transmittance and (c) emittance / absorption along (100) direction of single crystal 13.5 YSZ specimens with various thicknesses [80]

Figure 30 Emission energy with a black body spectrum at different temperatures 68

Figure 31 Temperature distributions calculated in a zirconia thermal barrier coating on the wall of a combustor compared with an opaque thermal barrier coating [83].

Figure 32 Schematic diagram of radiation intensity variation transporting through a ceramic coating.

Figure 33 Thermal conductivities of zirconia ceramic and zirconia thermal barrier coatings as a function of the yttria content [5]

Figure 34 Thermal conductivity of plasma-sprayed oxide cluster 9YSZ-Nd-Yb coatings and a baseline $\mathrm{ZrO}_{2}-4.55 \mathrm{~mol} \% \mathrm{Y}_{2} \mathrm{O}_{3}$ (4.55YSZ) coating as a function of test time

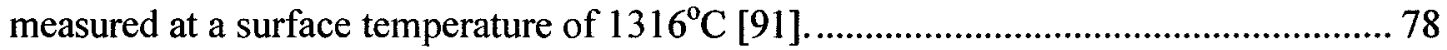

Figure 35 Zig-zag pores at different scale levels [92]. 79 xiv 
Figure 36 Layered structured EB-PVD TBC introduced by Plasma Assisted Physical Vapor Deposited (PAPVD) processing of the ceramic [5].

Figure 37 Thermal conductivities of layered EB-PVD TBC systems compared to the EB-PVD and thermal sprayed TBCs [5] 80

Figure 38 (a) Typical standard vapor phase columnar structure; and (b) modified columnar microstructure with multiple interfaces [46].

Figure 39 Thermal conductivity of EB-PVD coatings as a function of total number of layers produced by the "shutter" method, measured at various stages of testing, where $\mathrm{k}_{0}$ $=$ as deposited, $\mathrm{k}_{2}=$ after $2 \mathrm{hrs}$, and $\mathrm{k}_{5}=$ after $5 \mathrm{hrs}$ of testing [46].

Figure 40 Schematics of (a) the partial unit cell of the pyrochlore structure [95], (b) unit cell of fluorite structure. 83

Figure $41 \mathrm{ZrO}_{2}-\mathrm{LaO}_{2}$ phase diagram [98] exhibits stable pyrochlore structure at high temperatures. 83

Figure 42 Thermal conductivities of some pyrochlore compositions (compared to bulk YSZ) [99] 85

Figure 43 (a) Sectional view of a ceramic coating having metallic reflective layers; and (b) Temperature vs. distance from an exposed surface and illustrating the benefits attained by forming a protective coating [107]. 88

Figure 44 Hemispherical reflectance of $8 Y S Z / P t A 1 / M A R-M 247$ and effect of layering after 20 hours at $950^{\circ} \mathrm{C}[46]$. 88

Figure 45 Multiple layered $8 \mathrm{YSZ} / \mathrm{Al}_{2} \mathrm{O}_{3}$ structure with increased hemispherical reflectance [46]. 89 
Figure 46 Multilayered TBC increases IR reflectance with fixed and variable spacing $[46]$. 90

Figure 47 Flow chart for the fabrication of bulk materials with doped metal oxides..... 97

Figure 48 SEM image of powder size after ball milling (a) $20 \mathrm{hrs}$ and (b) $50 \mathrm{hrs}$ 101

Figure 49 Furnace used for sintering metal oxide co-doped zirconia based ceramics. 103

Figure 50 Temperature cycle for the sintering process. The holding time at $1500^{\circ} \mathrm{C}$ is

120 hours.

Figure 51 Different types of electrons originating from the interaction volume of the samples [118]. 108

Figure 52 Schematic diagram of x-rays diffracted by two adjacent rows of atoms in a crystal. 114

Figure 53 Schematic illustration of the heat flux DSC cell. .................................. 120

Figure 54 DSC temperature program with two heating cycles................................ 121

Figure 55 DSC equipment: Furnace /Specimen holder part [121] .......................... 121

Figure 56 (a) Schematic of the indentation testing for an ideal conical indenter and (b)

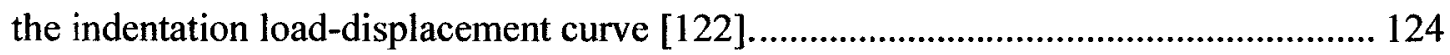

Figure 57 Schematic of laser flash diffusivity measurement.................................... 127

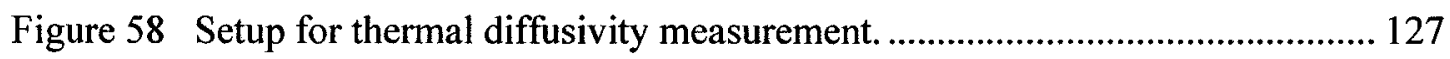

Figure 59 Equipment for measuring thermal diffusivity .......................................... 128

Figure 60 Morphologies of the as-received 7YSZ powders..................................... 130

Figure 61 Morphologies of the as-received (a) $\mathrm{Ta}_{2} \mathrm{O}_{5}$ powders; (b) $\mathrm{Nb}_{2} \mathrm{O}_{5}$ powders; (c) $\mathrm{Cr}_{2} \mathrm{O}_{3}$ powders; (d) $\mathrm{Sc}_{2} \mathrm{O}_{3}$ powders; (e) $\mathrm{Yb}_{2} \mathrm{O}_{3}$ powders; (f) $\mathrm{CeO}_{2}$ powders.................. 131

Figure 62 Particle morphologies of 7YSZ powders after 50hrs grinding.................... 132 xvi 
Figure 63 Morphologies of the alloyed powders after 50hrs grinding. (a) $3.9 \mathrm{TaYSZ}$ powder; (b) 3.9NbYSZ powder; (c) 5CrYSZ powder; (d) 5ScYSZ powder; (e) $5 \mathrm{YbYSZ}$ powder; and (f) $5 \mathrm{CeYSZ}$ powder. 134

Figure 64 SEM micrographs of sintered 7YSZ. Left image: fracture section; right image: sintered surface.

Figure 65 SEM micrographs of the sintered materials. Left image: fracture section; right image: sintered surface. 138

Figure 66 Estimation of grain size from the backscattered electron images of (a)

3.9TaYSZ and (b) 3.9NbYSZ. 140

Figure 67 EDX mapping of sintered 5CeYSZ. (a) SE image of the area being mapped;

(b) $\mathrm{Zr}$ mapping; and (c) Ce mapping. 145

Figure 68 EDX mapping of sintered 5ScYSZ. (a) SE image of the area being mapped;

(b) zirconium mapping; and (c) scandium mapping. 146

Figure 69 BEI images and element compositional analysis of doped materials: (a)

3.9TaYSZ; (b) 3.9NbYSZ; (c) 5CrYSZ; (d) 5ScYSZ; (e) 5YbYSZ; and (f) 5CeYSZ. . 153

Figure 70 XRD patterns of the sintered 7YSZ sample. 156

Figure 71 XRD patterns of the sintered 5CrYSZ sample.

Figure 72 XRD patterns of the sintered samples (a) 3.9TaYSZ; (b) 3.9NbYSZ......... 159

Figure 73 XRD patterns of the sintered samples (a) 5ScYSZ; (b) 5YbYSZ............... 160

Figure 74 XRD patterns of the sintered 5CeYSZ sample 161

Figure 75 Phase diagram for the $\mathrm{Y}_{2} \mathrm{O}_{3}-\mathrm{ZrO}_{2}$ binary system. The positions of eight doped samples studied are labeled in the phase diagram [25]. 165

xvii 
Figure 76 DSC curves for doped 7YSZ sintered materials upon heating and cooling at a

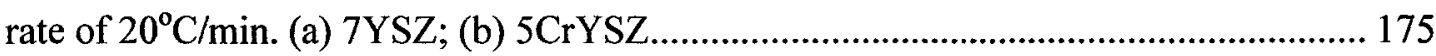
Figure 77 DSC curves for doped 7YSZ sintered materials upon heating and cooling at a rate of $20^{\circ} \mathrm{C} / \mathrm{min}$. (a) $3.9 \mathrm{TaYSZ}$; (b) $3.9 \mathrm{NbYSZ}$. 176 Figure 78 DSC curves for doped 7YSZ sintered materials upon heating and cooling at a rate of $20^{\circ} \mathrm{C} / \mathrm{min}$. (a) $5 \mathrm{ScYSZ}$; (b) $5 \mathrm{YbNbYSZ}$; and (c) $5 \mathrm{CeYSZ}$. 177 Figure 79 Specific heat of the doped samples (a) 7YSZ and 5CrYSZ; (b) 3.9TaYSZ and 3.9NbYSZ; and (c) 5ScYSZ, 5YbYSZ and 5CeYSZ. 179

Figure 80 The load-displacement curves of $5 \mathrm{YbYSZ}$ and 5CeYSZ samples. 181

Figure 81 Thermal diffusivities of $\mathrm{MO}_{1.5}(\mathrm{M}=\mathrm{Cr}$, Sc, and $\mathrm{Yb})$ and $\mathrm{CeO}_{2}$ doped $7 \mathrm{YSZ}$ as a function of temperature 184

Figure 82 Thermal diffusivity of $\mathrm{MO}_{2.5}(\mathrm{M}=\mathrm{Ta}, \mathrm{Nb})$ doped $7 \mathrm{YSZ}$ as a function of temperature.

Figure 83 Variations of thermal conductivity as a function of temperatures for 7YSZ and $5 \mathrm{MYSZ}(\mathrm{M}=\mathrm{Cr}, \mathrm{Sc}, \mathrm{Yb}$, and $\mathrm{Ce})$ samples. 186

Figure 84 Variations of thermal conductivity as a function of temperatures for 7YSZ and 3.9MYSZ $(\mathrm{M}=\mathrm{Ta}$ and $\mathrm{Nb})$ samples.

Figure 85 Thermal conductivities of pure $m-\mathrm{ZrO}_{2}[131]$ as a function of temperature. 197 Figure 86 Comparison of thermal conductivities between the calculated results and the measured results 201

Figure 87 Schematic of multiple layered coating structure containing two stacks $(T=$ transmitted, $\mathrm{R}=$ reflected and $\mathrm{I}=$ incoming radiations) 203

Figure 88 Transmission spectrum of 2 mm-thick sapphire $\left(\mathrm{Al}_{2} \mathrm{O}_{3}\right)$ window [138]..... 207 xviii 
Figure 89 Computed hemispherical reflectance of multiple layered coatings with 12 stacks.

Figure 90 Multiple layered coating structures. Structure A: monolayer; structure B: M (top) $+S_{1}+S_{2} ;$ structure $C: S_{1}\left(\right.$ top) $+M+S_{2} ;$ structure $D: M($ top $)+S_{1}+M+S_{2} \ldots . .211$ Figure 91 Schematic diagrams of radiation through the high reflectance multiple layered structures: (a) structure B; (b) structure C.

Figure 92 Computed temperature distributions for $250 \mu \mathrm{m}$ thick multiple layered coating structures with (a) lower scattering coefficient $\sigma_{\mathrm{s} \lambda}=1000 / \mathrm{m}$; (b) higher scattering coefficient $\sigma_{\mathrm{s} \lambda}=10000 / \mathrm{m}$ .228 Figure 93 Temperatures on the metal surface for structures A, B, C, and D under two scattering conditions.

Figure 94 Temperatures on the coating surface for structures A, B, C, and D under two scattering conditions.

Figure 95 Schematic diagrams of (a) deposition arrangement for obtaining the selected coating thickness of each layer and (b) vapor shield setup for the deposition of the $\mathrm{Al}_{2} \mathrm{O}_{3}$ / 7YSZ nanolayer coatings [38] 236

xix 


\section{Introduction}

The continual demand for increased gas turbine efficiency has pushed the superalloys to their upper limits of temperature capability and thermal stability. In today's engines, the hot gas temperatures in combustors have exceeded the incipient melting point of the Nibased superalloys by more than $250^{\circ} \mathrm{C}$ [1]. To reduce the temperatures of the superalloys and to prevent the structural failure of components due to melting, creep, oxidation, thermal fatigue and other modes of degradation, the continual development of air-cooling methods and the application of thermal barrier coatings (TBCs) to the hottest parts of the combustor and high-pressure turbine blades and vanes are two important approaches being continuously explored.

Turbine component air-cooling systems were developed in 1970s; in which the compressor discharge air is pumped through the cooling passageways in the blades and vane airfoils. With the improvement of casting processes and the use of well-developed air-cooling methods, high performance engines were able to operate at hot gas temperatures of 90 to $150^{\circ} \mathrm{C}$ higher than the melting points of the superalloys for thousands of hours [2]. However, cooling the airfoil with compressor air consumes energy to compress and pump the air through the cooling system, and results in loss of losing thrust and increased fuel consumption. In addition, the passageways inside the airfoils also increase the complexity of engines which makes them more difficult and expensive to manufacture. Thermal barrier coatings, on the other hand, with exceedingly low thermal conductivities, can provide thermal insulation to metal surfaces, reducing 
both metal surface temperatures and total heat flux into the solid metal. With the use of TBCs, the operating temperatures can potentially be increased by $70-150^{\circ} \mathrm{C}$ without any increase in metal temperatures, as shown in Figure 1[1]. Additional benefits are: extended lifetime of turbine blades can be achieved when the operating temperature remains unchanged; the design of turbine blades can be simplified by reducing the cooling flow requirements and further increasing the fuel efficiency $[3,4]$; and providing protection against thermal shock.

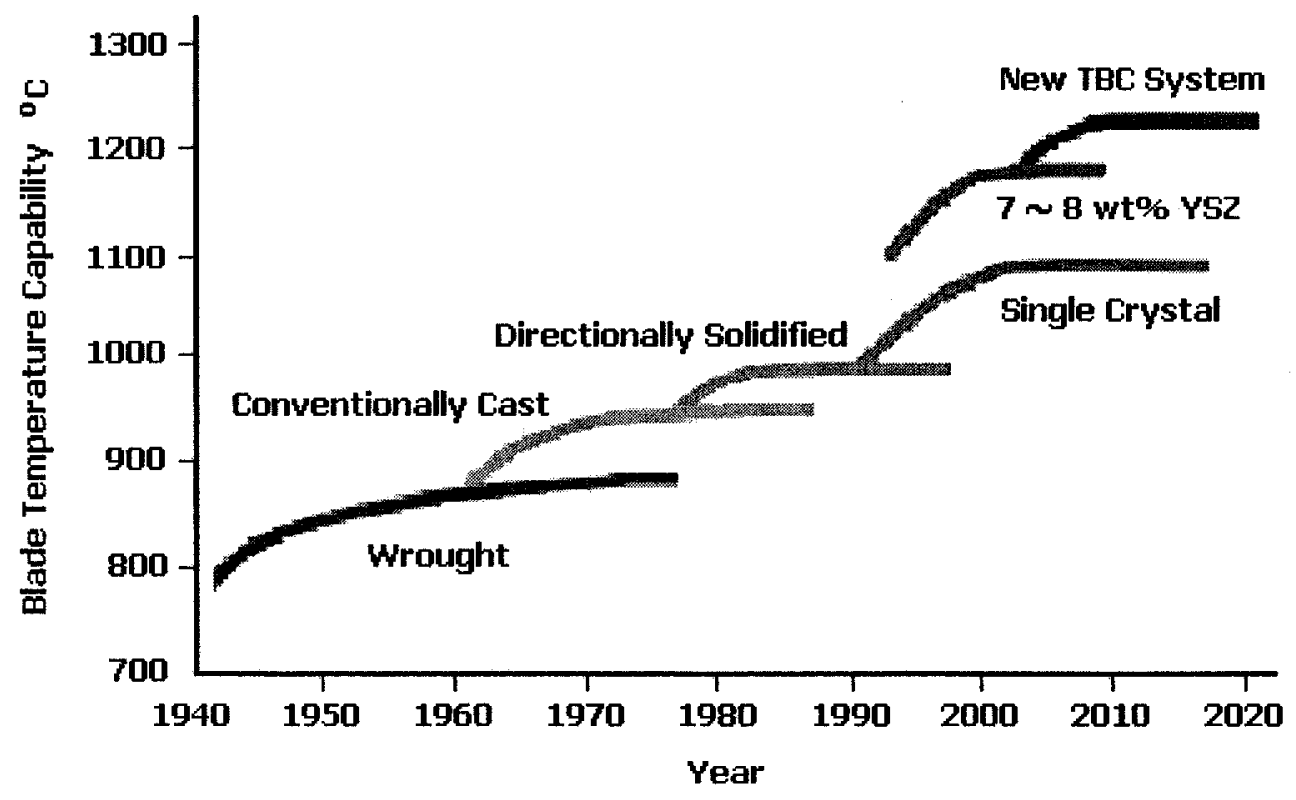

Figure 1 Increase in operating temperature of turbine components as a result of new superalloys, advanced manufacturing technology and thermal barrier coating development [1].

Further increases in engine operating temperatures necessitate the development and advancement of thermal barrier coatings. When ceramic TBC is applied, the temperature 
of superalloy components is realized via two mechanisms: reduced thermal conduction (phonon transport) and thermal radiation (photon transport). Thermal radiation is strongly dependent on temperature, being proportional to the fourth power. At the early stage of gas turbine development, thermal conduction is the dominant mechanism of heat transfer due to the relatively low gas temperatures. With the increase of gas temperatures, thermal radiation becomes increasingly important. For instance, at typical operating temperatures in today's engines, more than $90 \%$ radiation falls within the transparent region of yttria partially stabilized zirconia (YPSZ) coatings [5] and causes at least $50^{\circ} \mathrm{C}$ of additional temperature increase on metal substrates [6]. In recent years, tremendous efforts have been devoted to reduce thermal conductivities of the TBC materials by modifying their compositions, improving the deposition processes or microstructures of the coatings or developing new TBC materials. Research into microstructure and process modifications for the purpose of reducing photon transport through $\mathrm{TBC}$ system has recently received more attention. In modern engines, to increase the capability of thermal insulation of the coatings, both thermal conduction and thermal radiation should be considered. Hence, there is a need to design new thermal barrier coating systems where thermal conduction is reduced by selecting the coating materials with intrinsic low thermal conductivities, and thermal radiation is decreased by either increased reflectivity or increased scattering effects.

To reduce both thermal conduction and thermal radiation, this research was initiated with the two primary objectives: design a multiple layered ceramic structure with high reflectance to thermal radiation within the broadband wavelength range of $0.45 \sim 5 \mu \mathrm{m}$ so 
that the temperature increases caused by thermal radiation on both metal substrate and coating surface are minimized; and to optimize the TBC material of each layer to achieve the lowest thermal conductivity and appropriate optical parameters (refractive indices, scattering coefficients and absorption of the coatings) by doping yttria stabilized zirconia with metal oxides.

This thesis will be presented in the following order. A comprehensive literature review of the development of thermal barrier coating systems including deposition processes, materials, failure mechanisms, as well as heat transport mechanisms will be carried out. It will be followed by the experiments and studies of the properties of a series of metal oxide doped $7 \mathrm{wt} \%$ yttria stabilized zirconia (7YSZ) materials. A semi-empirical thermal conductivity model based on thermal conductivity theory and experimental results will be established to provide guidance on selection of TBC materials with low thermal conductivities. Finally, multiple layered coatings with high reflectance to thermal radiation will be proposed; and a novel TBC structure combining multiple layered stacks with high reflectance to thermal radiation and a single layered coating with low thermal conductivity will be presented. Furthermore, the temperature distributions within such structures will be calculated using energy equations and radiation transfer equations. 


\section{Literature Review}

\subsection{Thermal Barrier Coating Systems}

Typically TBCs are multiple layered systems, consisting of a top ceramic layer, a metallic bond coat layer situated between the ceramic layer and the superalloy substrate, superalloy substrate, and a very thin thermally grown oxide (TGO) layer formed at the ceramic/bond coat interface, as shown in Figure 2.

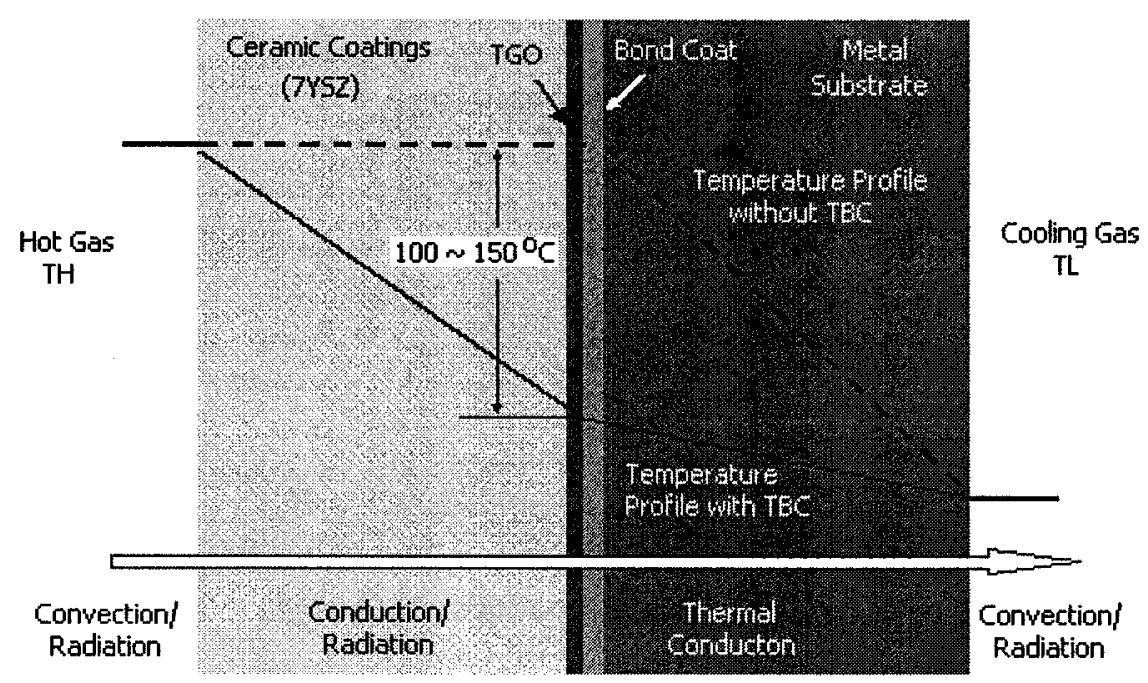

Figure 2 Typical Thermal Barrier Coating System.

The substrate is typically a nickel- or cobalt-based structural superalloy, which contains as many as 5 to 12 additional elements that are added to enhance specific properties, such as high temperature strength, ductility, oxidation resistance as well as phase stability [2]. As a highly integrated system, the properties of the superalloy have to be considered when selecting both ceramic topcoat and bond coat materials; for example, the thermal expansion coefficients of the ceramic topcoat and bond coat should match that of the 
superalloy, and there should be no or limited interdiffusion between the bond coat and the superalloy when operating at high temperatures.

The top ceramic layer, when directly exposed to hot gases, functions as thermal barrier and retards the flow of heat from the hot gas to the metal substrate so that the temperature of the superalloys can be kept low enough. Thus, the basic requirements for the top ceramic coatings are: 1) good thermal insulation, that is, low thermal conductivity and opaque to thermal radiation; 2) high melting point; 3) stable phases at operating temperatures; 4) appropriate thermal expansion coefficient which matches that of the superalloy substrate to reduce thermal stress; 5) erosion resistant to prevent impact damage caused by ingested particles such as sand and silicates in the environment of gas turbine engines; 6) oxidation and hot corrosion resistant; and 7) thermodynamically compatible with bond coat and TGO [7]. Considering all of these requirements, alloyed zirconium oxide has been found to be most suitable in its stabilized or partially stabilized form. The properties and failure mechanisms of topcoat ceramics are also affected by deposition processes, which result in different microstructures. Currently there are two techniques used for the deposition of ceramic top layers: plasma spraying and electron beam physical vapor deposition (EB-PVD), which will be discussed in detail in the following sections.

The metallic bond coat has two functions: they are to provide adhesion of the ceramic topcoat to the metal substrate and to protect the substrate from oxidation and high temperature corrosion since ziconia-based ceramics are oxygen transparent at high temperatures. These two functions can be realized by the formation of an $\alpha-\mathrm{Al}_{2} \mathrm{O}_{3}$ at the 
ceramic/bond coat interface. The bond coat materials should be selected to have the ability to provide a relatively large local $\mathrm{Al}$ reservoir such that alumina forms in preference to other oxides such as $\mathrm{NiO}$. In order to prevent premature failure of the TBC system by coating spalling, the bond coat must not form brittle phases, and have good resistance to interdiffusion with the superalloy substrate [7]. For these reasons, two types of bond coat alloys have been developed: one is platinum-modified nickel aluminide (PtNiAl) deposited by surface enrichment of $\mathrm{Al}$ via diffusion, which strongly interacts with the substrates and must therefore be tailored for each different superalloy substrate; and another is MCrAlY ( $\mathrm{M}=\mathrm{Ni}$, Co or NiCo, depending on the type of superalloy), which can be deposited by plasma spraying or EB-PVD. In a conventional MCrAIY bond coat, $\mathrm{Cr}$ provides hot-corrosion resistance, $\mathrm{Al}$ forms $\alpha-\mathrm{Al}_{2} \mathrm{O}_{3}$, and the role of $\mathrm{Y}$ is to improve the bond adhesion to the substrate. Based on one theory, the $\mathrm{Y}$ was found to combine with sulfur and prevent its segregation to the oxide layer, which is detrimental to its adhesion $[8,9]$.

The formation of TGO, a thin layer between the ceramic topcoat and the bond coat, is the result of thermal oxidation of the $\mathrm{Al}$ in the bond coat due to the oxygen transparency of the zirconia based TBC material. The growth rate of the oxide is controlled by inward diffusion of $O$. Generally, the inward diffusion of oxygen through the TGO controls further growth of TGO into the bond coat, but in some instances, TGO growth is controlled by outward diffusion of $\mathrm{Al}$, leading to the formation of a new TGO at the TGO/topcoat interface or at the $\alpha-\mathrm{Al}_{2} \mathrm{O}_{3}$ grain boundaries within the TGO. An ideal bond coat should ensure the formation of a thin, uniform and defect free $\alpha-\mathrm{Al}_{2} \mathrm{O}_{3}$ layer 
(TGO) at the ceramic/bond coat interface. Of all possible oxides, the $\alpha-\mathrm{Al}_{2} \mathrm{O}_{3}$ is preferred due to its very low oxygen ionic diffusivity, which provides an excellent diffusion barrier to oxygen transport at high temperatures and therefore improves oxidation resistance [10]. In addition, $\alpha-\mathrm{Al}_{2} \mathrm{O}_{3}$ phase is compatible with yttria stabilized zirconia topcoat, which ensures long-term thermodynamic stability of the coating systems [7]. It has been found that the growth rate and thickness of TGO are very critical for the performance of thermal barrier coating systems [7], and these properties of TGO are strongly affected by the specific composition of the bond coat. For instance, the optimization of yttium content has been found to be very important in providing adhesion of the ceramic and avoiding rapid spalling of TBCs, and further extending the service life of TBCs $[11,12]$.

For application in aircraft engines, especially rotating turbine blade, the thickness of the coating is a very important parameter for engine design. Although the thicker the coatings, the better the thermal insulation, it also increases the component weight and may accelerate the spallation of coatings due to the thermal stresses. A burner rig test to determine the effects of ceramic thickness on cyclic durability was conducted by Pratt \& Whiteney and found that with increased thickness of ceramic, the temperature of the top surface increases, introducing a sintering effect. "Sintering and associated shrinkage introduce tensile stresses which result in transverse cracking of the ceramic perpendicular to the interface" [14]. To balance the good thermal insulation and the weight of the coatings, the thickness of the ceramic top layer on turbine blades was limited to $250 \mu \mathrm{m}$, and that of the bond coat was $125 \mu \mathrm{m}$. 


\subsection{The Evolution of Thermal Barrier Coatings Systems}

Since the idea of applying a thin thermally insulating ceramic layer on the metal surface to reduce the temperature of superalloys was first developed by NASA and US Air Force research in the late $1950 \mathrm{~s}$ [2], thermal barrier coatings have been used and further developed for almost five decades. As shown in Figure 3, the evolution of TBC systems has been associated with the development of top ceramic materials, bond coat materials as well as the deposition processes for the top layer and bond coat.

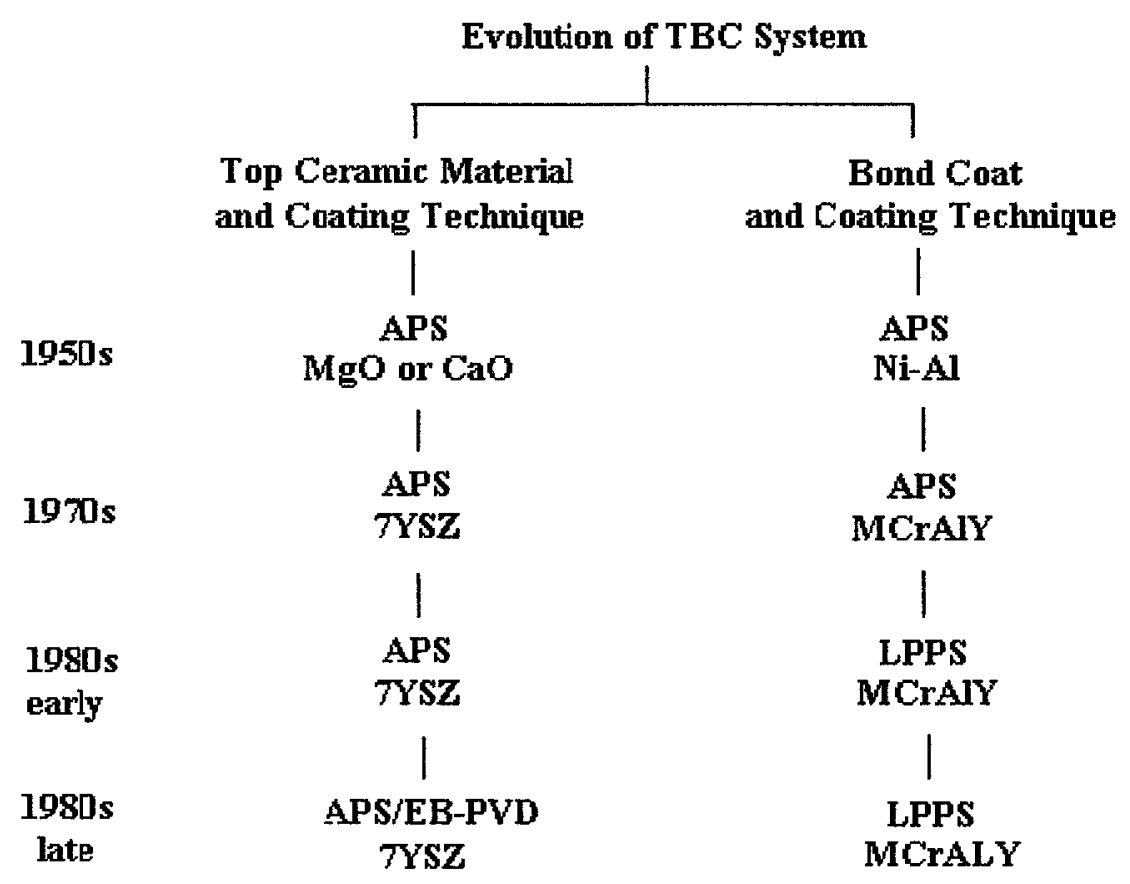

Figure 3 Evolution of TBC systems [14].

The early thermal barrier coating systems consist of an air plasma-sprayed $22 \mathrm{wt} \% \mathrm{MgO}$ or $\mathrm{CaO}$ fully stabilized $\mathrm{ZrO}_{2}$ ceramic $(22 \mathrm{MgSZ}$ or $\mathrm{CaSZ})$ top layer and an air plasmasprayed $\mathrm{Ni}-\mathrm{Cr}$ or $\mathrm{Ni}-\mathrm{Al}$ inner metallic bond coat, which was applied on combustor chamber walls and burner cans for the protection of combustors and augmentors from 9 
oxidation damage and to increase the temperature capability of components [13]. However, with the increase in operating temperature, $\mathrm{MgO}$ stabilized $\mathrm{ZrO}_{2}$ became inadequate due to its crystallographic destabilization at temperatures above $954^{\circ} \mathrm{C}$. The maximum service temperature of the plasma-sprayed $\mathrm{MgO}$ stabilized $\mathrm{ZrO}_{2}$ coating is in the order of $982^{\circ} \mathrm{C}$. Therefore, a $7 \mathrm{wt} \% \mathrm{Y}_{2} \mathrm{O}_{3}$ partially stabilized $\mathrm{ZrO}_{2}$ (7YSZ) was introduced to replace $22 \mathrm{MgSZ}$. In fact, in the mid $70 \mathrm{~s}, 7 \mathrm{YSZ}$ had been developed for applications on turbine airfoils; and as a result, the overall engine performance had been improved significantly. When 7YSZ TBCs were applied to combustors, four times improvement in durability was achieved.

During this period, the bond coat material also experienced an evolution and air plasma sprayed Ni-Al was replaced by air plasma sprayed NiCoCrAlY. At Pratt \& Whitney, this advancement in TBC materials was classified as the first generation of such materials. However, at such high temperatures, the bond coat oxidation became serious due to the deposition process of air plasma spraying. The failure for generation I materials usually occurred within the bond coat with the appearance of so-called "black failure". This was due to the pre-existence of oxide nuclei formed during air plasma spraying of the NiCoCrAlY bond coat, which favors the growth of NiO. This oxide is voluminous and relatively weak in comparison with the thin oxide that forms on alumina formers. Therefore, upon thermocyclic exposure, biaxial in-plane compressive stresses developed within the oxide. When these stresses exceeded the compressive strength of the oxidized bond coat, failure occurred within the bond coat giving the black appearance of the oxide [14]. 
To overcome the problem of failure associated with the bond coat for the generation I, low-pressure chamber plasma sprayed (LPPS) NiCoCrAIY bond coat was developed in 1982 to strengthen the bond coat, where the absence of oxygen during deposition of the bond layer eliminated the pre-existing oxide nuclei to a great extent. This process development marked the inception of the second generation of TBC systems. This development has made it possible for TBCs to be applied on vane airfoil surfaces and platforms, with operating environment structurally and thermally more severe than that experienced by the combustor. Thus a 2.5 times improvement of durability over generation I was achieved [13]. Since the second generation focused on strengthening the bond coat, the weak link became the ceramic layer itself. The failure mechanism was the spallation of the ceramic topcoat which gave rise to the "white failure" appearance. Cracks developed and propagated parallel to and near to the bond coat/ceramic topcoat interface, however, it always remained within the ceramic [14].

In 1987, the exploitation of strain-tolerant TBCs gave rise to a renaissance of industrial EB-PVD technology, which had been previously utilized for depositing metallic MCrAIY coatings for more than 20 years. Thus, the third generation TBC systems emerged with 7YSZ ceramic topcoat deposited by EB-PVD and NiCoCrAlY metallic bond coat deposited by LPPS. A roughly three fold improvement in turbine blade life, or a surface temperature increase on the hot section of foils of approximately $150^{\circ} \mathrm{C}$ was achieved $[14,15]$. The failure mode for the third generation TBC is the spallation of ceramic due to the propagation of cracks either within the TGO or at the TGO-bond coat interface [14]. More details will be given in section 2.5. 
Due to the economics and ease for manufacturing, plasma-sprayed YSZ TBC is currently the material of choice on many commercial jet engine parts; a more durable EB-PVD deposited ceramic coating is the bill-of-material on turbine blades and vanes in current high thrust engine models [13].

\subsection{Zirconia Based TBC Materials}

The properties of high melting temperature, higher thermal expansion coefficient, low thermal conductivity and good erosion resistance have made zirconia $\left(\mathrm{ZrO}_{2}\right)$ the favorite TBC material. However, pure zirconia is rarely used due to the need to manufacture and to store coated parts at room temperature. Upon cooling from the deposition temperature, the phase transformation of $\mathrm{ZrO}_{2}$ from tetragonal to monoclinic results in spontaneous cracking. To prevent this failure as well as to improve specific properties, metal oxides are added to the $\mathrm{ZrO}_{2}$ matrix to delay the transformation from tetragonal to monoclinic phase. In this section, the crystal structures, phase transformations as well as the properties of zirconia based ceramic materials will be thoroughly described.

\subsubsection{Pure Zirconia $\left(\mathrm{ZrO}_{2}\right)$}

At ambient pressure, pure zirconia can assume three phases, depending on the temperatrue. Cubic zirconia $\left(c-\mathrm{ZrO}_{2}\right)$, a high temperature phase existing at temperatures higher than $2300^{\circ} \mathrm{C}$, has a simple fluorite structure with a simple cubic anion $\left(\mathrm{O}^{2-}\right)$ sublattice and a face-centered cubic cation $\left(\mathrm{Zr}^{4+}\right)$ sublattice. The combination of these two sublattices shows an eight fold coordination for the $\mathrm{Zr}^{4+}$ cations, as shown in Figure 4. 


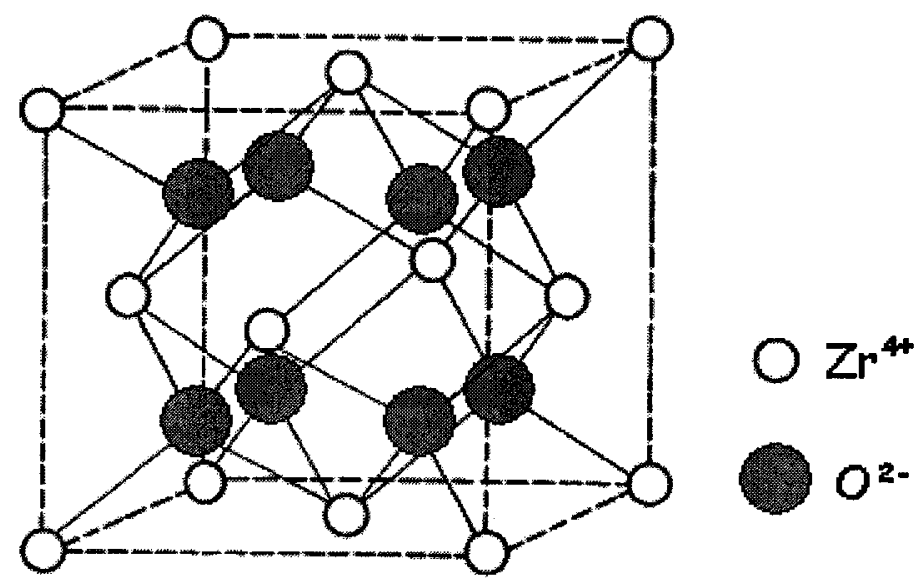

Figure 4 Fluorite structure showing the eightfold coordination of the cubic $\mathrm{ZrO}_{2}$ [16].

When temperature is decreased, $\mathrm{ZrO}_{2}$ undergoes a cubic to tetragonal $(c \rightarrow t)$ phase transition at around $2300^{\circ} \mathrm{C}$, where the displacement of oxygen ions from the fluorite site results in the occurrence of cell doubling and the fluorite cubic structure distorts to the tetragonal structure with the tetragonal $c$-axis parallel to the cubic $<001>$ axes [17]. Figure 5 shows the crystal structures of two face centered tetragonal unit cells with a body centered tetragonal cell.

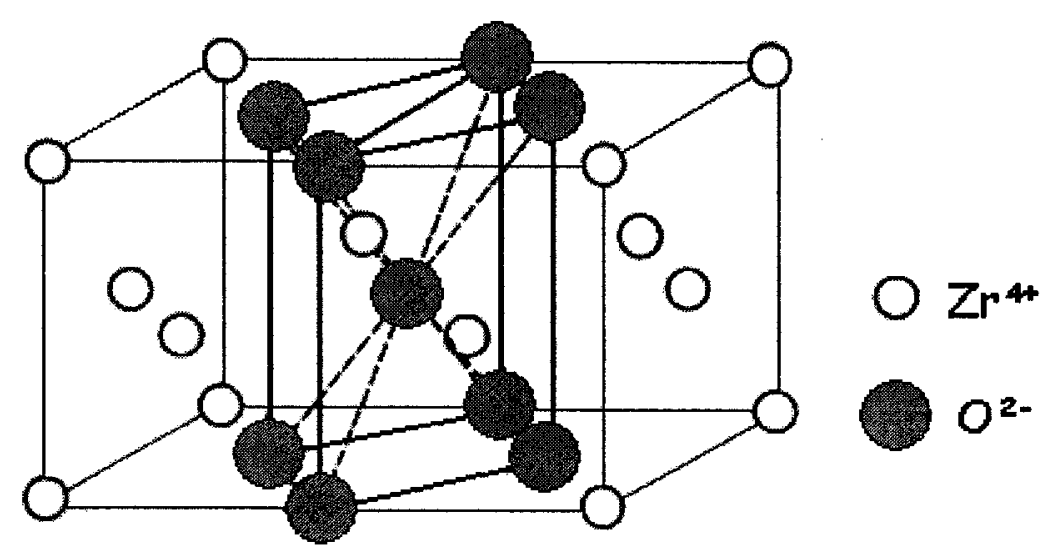

Figure 5 Crystal structure of two face-centered tetragonal zirconia [16].

When temperature is further decreased to $1205^{\circ} \mathrm{C}$, the tetragonal phase becomes unstable 13 
and transforms to monoclinic phase $\left(m-\mathrm{ZrO}_{2}\right)$. The crystal structure of the monoclinic $\mathrm{ZrO}_{2}$ has been observed to be a distortion of the cubic fluorite type, and the $\mathrm{Zr}$ atom is in sevenfold coordination [18], as shown in Figure 6. During the phase transformations, it was observed in TEM work that the monoclinic $c$ axis $\left(c_{m}\right)$ is parallel to the tetragonal $c$ axis $\left(c_{t}\right)$ below $1000^{\circ} \mathrm{C}$ and the monoclinic $b$ axis $\left(b_{m}\right)$ is parallel to the tetragonal $c$ axis $\left(c_{t}\right)$ when above $1000^{\circ} \mathrm{C}[19]$. In the monoclinic phase the translational periodicities along the $a_{t}$ and $b_{t}$ directions become twice those in the tetragonal phase as a result of the tetragonal to monoclinic phase transition, i.e., a cell doubling occurs. Therefore, this $t-$ $m$ transformation involves a discontinuous volume increase, which results in destructive cracking of the bulk crystal. The lattice parameters of various phases of pure zirconia are summarized in Table 1.

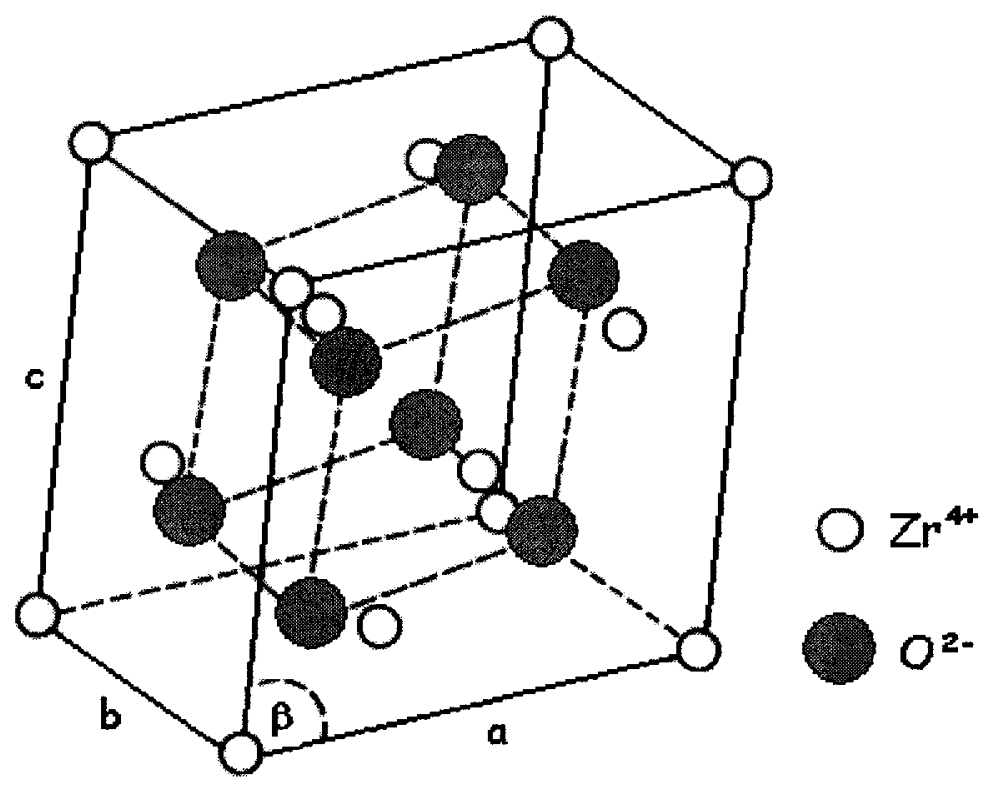

Figure 6 Monoclinic structure of $\mathrm{ZrO}_{2}$ [16]. 
Table 1 Lattice parameters of pure zirconia for three phases [20]

\begin{tabular}{|l|l|l|l|l|l|}
\hline \multirow{2}{*}{ Phase } & \multirow{2}{*}{ Stability } & \multicolumn{4}{|l|}{ Cell parameters $(\AA)$ (Extrapolated to room temperature) } \\
\cline { 3 - 6 } & range $\left({ }^{\circ} \mathrm{C}\right)$ & $\boldsymbol{a}$ & $b$ & $c$ & $\beta$ \\
\hline Cubic & $2377-2710$ & 5.117 & 5.117 & 5.117 & $90^{\circ}$ \\
\hline Tetragonal & $1205-2377$ & 5.074 & 5.074 & 5.188 & $90^{\circ}$ \\
\hline Monoclinic & $0-1205$ & 5.1507 & 5.2028 & 5.3156 & $99.194^{\circ}$ \\
\hline
\end{tabular}

\subsubsection{Zirconia Alloys}

\subsubsection{Mechanisms of phase stabilization}

In order to utilize $\mathrm{ZrO}_{2}$ at room temperature without cracking, it is desirable to retain the high temperature phase to room temperature by lowering the phase transformation temperature. The thermodynamic analysis by Bocanegra-Bernal et al. [19] indicated that the tetragonal to monoclinic phase transformation is governed by the free-energy change of the entire system, $\Delta G_{t \rightarrow m}$, which depends on the chemical free-energy change, $\Delta G_{c}$, the strain energy change, $\Delta U_{s e}$, and the energy change associated with the surface of the inclusion $\Delta U_{s}$, i.e.:

$\Delta G_{t \rightarrow m}=-\left|\Delta G_{c}\right|+\Delta U_{s e}+\Delta U_{s}$

(Eq. 1)

From (Eq. 1), it can be seen that a decrease in $\Delta \mathrm{G}_{c}$ will result in the reduction of the freeenergy change $\Delta G_{t \rightarrow m}$, and therefore the transformation temperature can be reduced so 
that the cubic or tetragonal phase can be stabilized to room temperature.

It was suggested that the instability of $\mathrm{t}-$ or $\mathrm{c}-\mathrm{ZrO}_{2}$ at room temperature could be attributed to the interaction between the neighboring oxygen ions, which could be altered by either increasing the lattice parameters or introducing oxygen vacancies to the crystal [21]. Therefore, the existence of oxygen vacancies or larger substituting cations could play a role in the phase stability and phase transformations of zirconia polycrystals [22]. Especially the anion vacancies are thought to be largely responsible for the stabilization of the high temperature tetragonal and cubic $\mathrm{ZrO}_{2}$. The mechanism whereby oxygen vacancies stabilize the high temperature phase can be explained by the coordination number of zirconium. The coordination number of a zirconium atom in tetragonal and cubic $\mathrm{ZrO}_{2}$ is eight, while in the monoclinic phase it is seven. That is, the zirconium atoms have a tendency for a co-ordination number lower than eight at room temperature, which makes the tetragonal or cubic phase unstable [21]. The introduction of oxygen vacancies enables the zirconium cation to be surrounded by seven oxygen anions instead of eight in the fluorite structures. Therefore, the lower effective coordination number of the zirconium atom stabilizes the fluorite structure to room temperature. On the other hand, the substitution of zirconium with larger cations, such as $\mathrm{Ca}, \mathrm{Ce}$ and $\mathrm{Y}$ for $\mathrm{Zr}$ will increase the lattice parameters, which enables the eight coordination of oxygen anion to be stable at room temperature.

When a metal oxide or dopant with a cation valence less than that of $\mathrm{Zr}$ atom is added to zirconia, either cation interstitials or anion vacancies must form in order to maintain charge balance. For doped zirconia, the preferred structure is that the cation $\mathrm{Zr}^{4+}$ is 
replaced by the cation of the dopant instead of forming cation interstitials since the reaction energy to form an oxygen vacancy is much lower than that for a cation interstitial [34]. For example, in the $\mathrm{CaO}-\mathrm{ZrO}_{2}$ system, the reaction energy for the formation of an oxygen vacancy is $0.88 \mathrm{eV}$ compared with $10.05 \mathrm{eV}$ for a cation interstitial. Substantial experiments have verified that the oxide cations are incorporated by substituting for zirconium on the fluorite cation sites, and the charge balance is achieved through the formation of oxygen vacancies. For dopants with valence less than +4 , oxygen vacancies are generated within the ionic lattice to maintain electrical neutrality. The X-ray absorption studies of polycrystalline zirconia showed that every single charge-compensating oxygen vacancy is generated by two substitutional yttrium atoms and the oxygen vacancy is preferentially located as nearest neighbors of $\mathrm{Zr}^{4+}$, leaving eightfold coordination to $\mathrm{Y}^{3+}$ ion [24].

In summary, to stabilize cubic or tetragonal $\mathrm{ZrO}_{2}$ to room temperature, either the number of oxygen ions around the zirconium cations must be reduced by creating oxygen vacancies, or the lattice parameters must be increased by doping cations with ionic radii larger than that of zirconium cation so that a structure with coordination number eight is produced. Based on these rules, metal oxides with larger ionic size and lower valence, such as $\mathrm{MgO}, \mathrm{CaO}, \mathrm{CeO}_{2}, \mathrm{Y}_{2} \mathrm{O}_{3}, \mathrm{Yb}_{2} \mathrm{O}_{3}$, and $\mathrm{Sc}_{2} \mathrm{O}_{3}$, have been proven to be effective stabilizers [23].

\subsubsection{Phase diagram of the yttria - zirconia system}

Figure 7 is a portion of the binary equilibrium phase diagram for zirconium oxide $\left(\mathrm{ZrO}_{2}\right)$ 
and yttrium oxide $\left(\mathrm{Y}_{2} \mathrm{O}_{3}\right)$. It is to be noted that the $\mathrm{x}$-axis represents $\mathrm{YO}_{1.5}$ which is one half of $\mathrm{Y}_{2} \mathrm{O}_{3}$. The horizontal axis extends to about $10 \mathrm{~mol} \% \mathrm{Y}_{2} \mathrm{O}_{3}$. From Figure 7, it can be found that the pure $\mathrm{ZrO}_{2}$ has three crystal forms, a cubic structure stable at the highest temperature range, between the melting point $\left(2680^{\circ} \mathrm{C}\right)$ and $2370^{\circ} \mathrm{C}$, a tetragonal phase stable at intermediate temperature range of 2370 to $1170^{\circ} \mathrm{C}$, and a monoclinic form stable at temperatures below $1170^{\circ} \mathrm{C}$. Upon cooling, pure $\mathrm{ZrO}_{2}$ experiences cubic to tetragonal and tetragonal to monoclinic phase transformations.

The phase diagram shows that the addition of yttrium oxide to zirconium oxide extends the existence of cubic and tetragonal phases to lower temperatures. With increasing $\mathrm{Y}_{2} \mathrm{O}_{3}$ content, the phase transformation temperature from tetragonal to monoclinic decreases. Over the range of $2.5-7.5 \mathrm{~mol} \% \mathrm{Y}_{2} \mathrm{O}_{3}$ and above $600^{\circ} \mathrm{C}$ there is a two-phase region: both cubic and tetragonal phases are present. At room temperature, the equilibrium phases consist of monoclinic and cubic phase. In this situation, the zirconia material is termed a partially stabilized zirconia. When the $\mathrm{Y}_{2} \mathrm{O}_{3}$ content exceeds $7.5 \mathrm{~mol} \%$, the cubic phase will remain to room temperature. Thus, this material is called fully stabilized zirconia.

For the mixture of 3 5 mol\% $\mathrm{Y}_{2} \mathrm{O}_{3}$ with $\mathrm{ZrO}_{2}$, the structures at room temperature depend on the cooling rate and may assume non-equilibrium forms. For example, $3 \mathrm{~mol} \% \mathrm{Y}_{2} \mathrm{O}_{3}$ stabilized $\mathrm{ZrO}_{2}$ rapidly cooled from $2200^{\circ} \mathrm{C}$ experiences a diffusionless phase transformation and forms a metastable phase, tetragonal prime $\left(t^{\prime}\right)$ phase, to room temperature. The zirconia alloys with metastable phases are thermodynamically unstable, but kinetically, the transformation to the monoclinic phase does not occur at room temperature. At the bottom of the diagram, the dashed lines indicate fields in which 
metastable tetragonal and cubic phase may exist. Both the martensitic or diffusionless transformations may occur upon rapid cooling. This metastable $\mathrm{ZrO}_{2}$ is of great interest in high temperature applications as TBC material. Thus knowledge about the properties of metastable $\mathrm{ZrO}_{2}$ is very desirable.

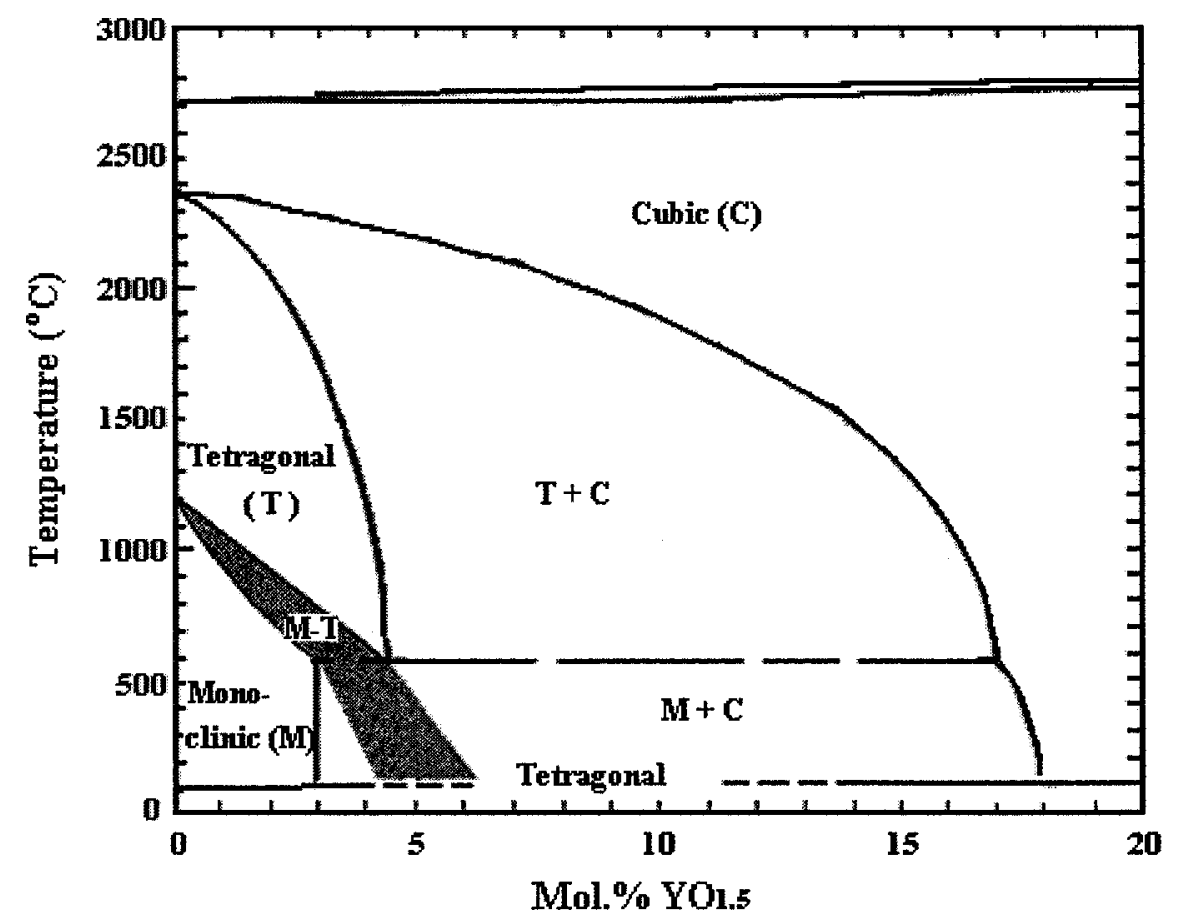

Figure 7 The pseudo-binary $\mathrm{YO}_{1.5}-\mathrm{ZrO}_{2}$ phase diagram. The labeling across the bottom of the diagram shows metastable phase fields upon rapid cooling [25].

\subsubsection{Lattice parameters of stabilized $\mathrm{ZrO}_{2}$}

The lattice parameters of stabilized $\mathrm{ZrO}_{2}$ have been thoroughly studied $[23,25,26,27]$. It has been found that the lattice parameters vary with the composition, ionic radius and valence of dopant cations, as well as the crystal structure. Using the whole-powderpattern decomposition (WPPD) technique, the unit cell parameters of doped $\mathrm{ZrO}_{2}$ at low 
content of $\mathrm{YO}_{1.5}$ was measured and the relationship between the lattice parameters of monoclinic $\mathrm{ZrO}_{2}$ and $\mathrm{YO}_{1.5}$ content for the monoclinic structure was found to be [26]:

$a=0.51471+0.000376 x$
$b=0.52111+0.000037 x$
$c=0.53144+0.000208 x$
$\beta=99.217-0.0432 x$

(Eq. 2)

where $x$ is the mole percentage of $\mathrm{YO}_{1.5}$ content within the range of $x \leq 0.04$. The lattice parameter variations of cubic and tetragonal $\mathrm{ZrO}_{2}$ with the composition of dopant oxide in the $\mathrm{Y}_{2} \mathrm{O}_{3}-\mathrm{ZrO}_{2}$ system were [25]:

$a=5.1159+0.1547 x \quad$ Cubic $\mathrm{ZrO}_{2}$

(Eq. 3)

where $x$ is the mole percentage of $\mathrm{YO}_{1.5}$ content in the alloy within the limits $0.12<x<0.25$. And:

$a=5.0801+0.3582 x$

$c=5.1944-0.3045 x$

Tetragonal $\mathrm{ZrO}_{2}$

(Eq. 4)

where $x$ is the mole percentage of $\mathrm{YO}_{1.5}$ content in the alloy within the limits $0.04<x<0.13$

The temperature response of $\mathrm{ZrO}_{2}$ has also been studied and has shown that the thermal expansion coefficient $\alpha$ curves at different compositions could be expressed by the relationship [27]:

$\alpha=5.1169+0.1526 x+4.6468 \cdot 10^{-5}(T-273)+7.6613 \cdot 10^{-5}(T-273)$

(Eq. 5) 
where $x$ is the mole percentage of $\mathrm{YO}_{1.5}$ content and $T$ is the temperature in $\mathrm{K}$.

In summary, the lattice parameters for the cubic, tetragonal and monoclinic phases as a function of mole percentage of $\mathrm{YO}_{1.5}$ are shown in Figure 8 [20], where the $b$-axis appears not to vary greatly with the mole percentage of $\mathrm{YO}_{1.5}$. It can be seen that the lattice parameters of monoclinic $\mathrm{ZrO}_{2}$ exhibit greater expansion than those of tetragonal $\mathrm{ZrO}_{2}$, which results in a destructive stress within the crystal when the phase is transformed from tetragonal to monoclinic.

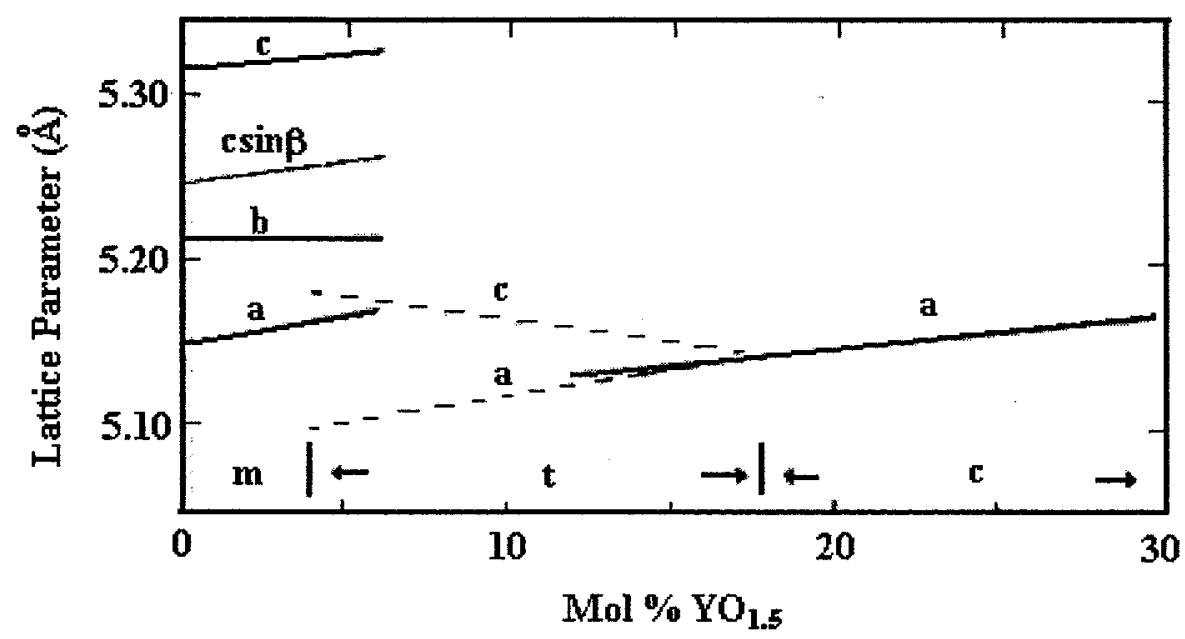

Figure 8 Lattice parameters for the cubic, tetragonal and monoclinic phases as a function of $\mathrm{mol}_{\mathrm{O}} \mathrm{YO}_{1.5}[20]$.

\subsubsection{Microstructures and Properties of Tetragonal $\mathrm{ZrO}_{2}\left(t^{\prime}\right.$ phase)}

When partially stabilized zirconia with metal oxide dopants is rapidly cooled from high temperature to room temperature, diffusion does not have time to occur. The supercooled alloys may either retain their cubic structure or undergo diffusionless transformations to a twinned tetragonal phase $\left(t^{\prime}\right)$ [17]. This quenched tetragonal phase $t^{\prime}$ is 21 
crystallographically the same as $t$ but with a higher yttria content and a lower c/a ratio, and is normally considered metastable [20]. This metastable $t$ ' phase is also called nontransformable phase; it cannot transform into monoclinic phase by the martensitic transformation process.

The microstructure of of $\mathrm{ZrO}_{2}-4 \mathrm{~mol}_{2} \mathrm{Y}_{2} \mathrm{O}_{3}$ formed by rapid solidificaiton after arcmelting is shown in Figure 9 [17]. The bright field image exhibits a plate-like microstructure, which is one of the forms of $t^{\prime}-\mathrm{ZrO}_{2}$. In the dark field image, curved boundaries with antiphase domain boundaries, termed as domain structure, are some of the characteristics of $t^{\prime}-\mathrm{ZrO}_{2}$ formed by the diffusionless c-t transformation. T. Sakuma [17] examined the microstructures of arc-melted $\mathrm{ZrO}_{2}-4 m o l \% \mathrm{R}_{2} \mathrm{O}_{3}$ and $\mathrm{ZrO}_{2}-$ $8 \mathrm{~mol} \% \mathrm{R}_{2} \mathrm{O}_{3}$ (where $\mathrm{R}$ represents rare earth elements: $\mathrm{Gd}, \mathrm{Y}, \mathrm{Er}, \mathrm{Yb}, \mathrm{Nd}$ and $\mathrm{Sm}$ ). It was found that cations of $\mathrm{Gd}, \mathrm{Y}, \mathrm{Er}, \mathrm{Yb}$ induced formation of domain structures with platelike twins; whereas cations of $\mathrm{Nd}$ and $\mathrm{Sm}$ induced formation of thin banded structures, with curved interfaces between the bands. Each band includes fine granular structures. The differences in microstructures were explained as follows: The initial structure of $\mathrm{ZrO}_{2}$ consists of three $t^{\prime}-\mathrm{ZrO}_{2}$ variants and is unstable due to the extremely large boundary area. Therefore some $t^{\prime}-\mathrm{ZrO}_{2}$ grows rapidly at the expense of others - domain structure is developed - twins are induced to accommodate the strain in zirocnia with cations of Gd, $\mathrm{Y}, \mathrm{Er}$, and $\mathrm{Yb}$. On the other hand, when cations of $\mathrm{Nd}$ and $\mathrm{Sm}$ are added to zirconia, cooling rates may not be high enough to suppress diffusion of ions, and localized diffusion may originate the stable fine structure, as illustrated in Figure 10.

It has been reported that the $t^{\prime}-\mathrm{ZrO}_{2}$ has excellent strength and cracking resistance at high 
temperatures. This high temperature stability and mechanical properties of $t^{\prime}-\mathrm{ZrO}_{2}$ are due to its ferroelastic domain-switching mechanism $[2,28]$. However, when the $t^{\prime}-$ $\mathrm{ZrO}_{2}$ alloy is reheated above $1200^{\circ} \mathrm{C}$, into the $c+t$ two-phase region, to reach the equilibrium state, the $t^{\prime}$ phase will decompose into a cubic phase with higher content of doping ions and a tetragonal phase with lower content of doping ions. On quenching the two-phase alloy to room temperature, the tetragonal phase may experience a martensitic phase transition and transform into monoclinic phase while the cubic phase retains fluorite structure to room temperature due to its higher percentage of doping ions.
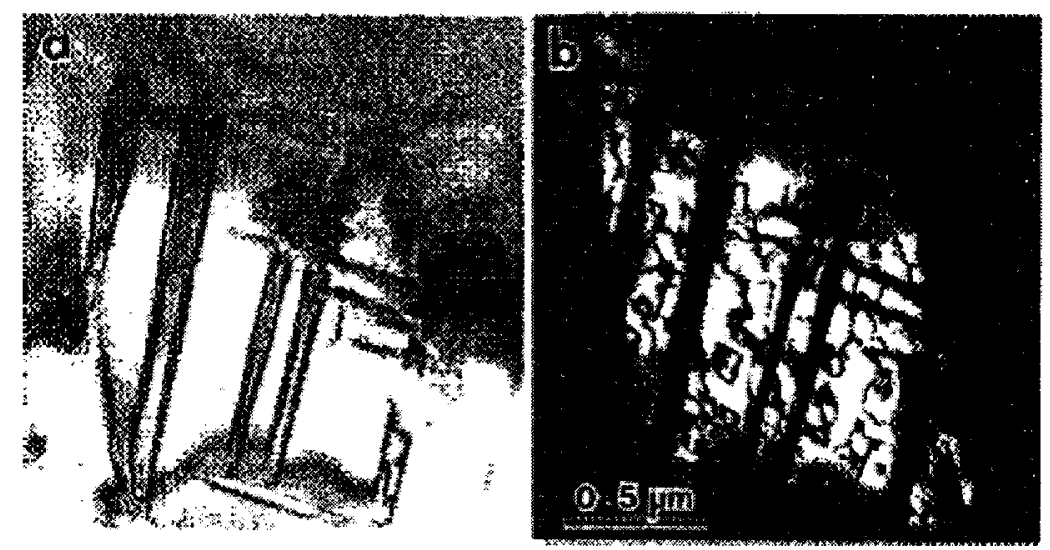

Figure 9 Microstructures of $\mathrm{ZrO}_{2}-4 \mathrm{~mol}_{2} \mathrm{Y}_{2} \mathrm{O}_{3}$ prepared by arc-melting; (a) bright field image and (b) dark field image taken with a (112) reflection [17].

It was proposed [29] that there is a minimum dopant content which renders the microstructure "non-transformable". The minimum percentage of dopant for both small (Sc) and large (La) cations is higher than that of Y. The phase stability of doped zirconia with constant dopant concentration $(7.6 \mathrm{~mol} \%)$ shows that $\mathrm{Yb}, \mathrm{Y}$ are optimal. This is because the cation sizes of $\mathrm{Yb}$ and $\mathrm{Y}$ interestingly match the size of the coordinating oxygen cube, as shown in Figure 11. 

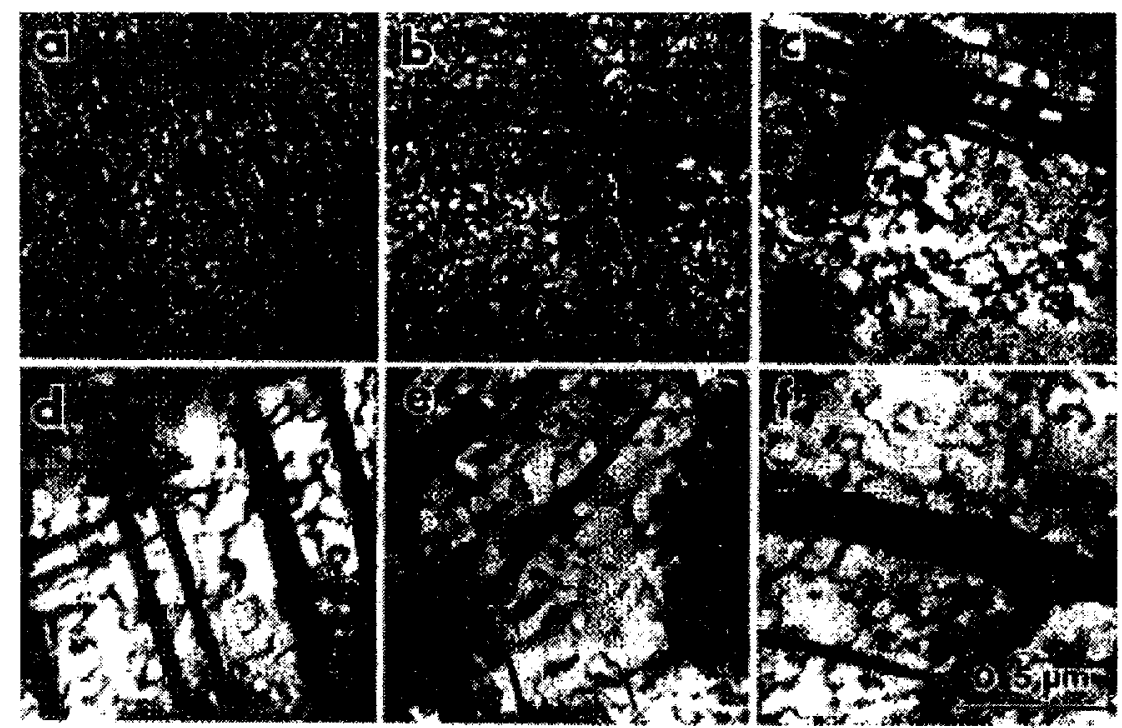

Figure 10 The microstructure in $\mathrm{ZrO} 2-4$ mol\% (a) $\mathrm{Nd}_{2} \mathrm{O}_{3}$, (b) $\mathrm{Sm}_{2} \mathrm{O}_{3}$, (c) $\mathrm{Gd}_{2} \mathrm{O}_{3}$, (d) $\mathrm{Y}_{2} \mathrm{O}_{3}$, (e) $\mathrm{Er}_{2} \mathrm{O}_{3}$ and (f) $\mathrm{Yb}_{2} \mathrm{O}_{3}$ prepared by arc-melting [17].

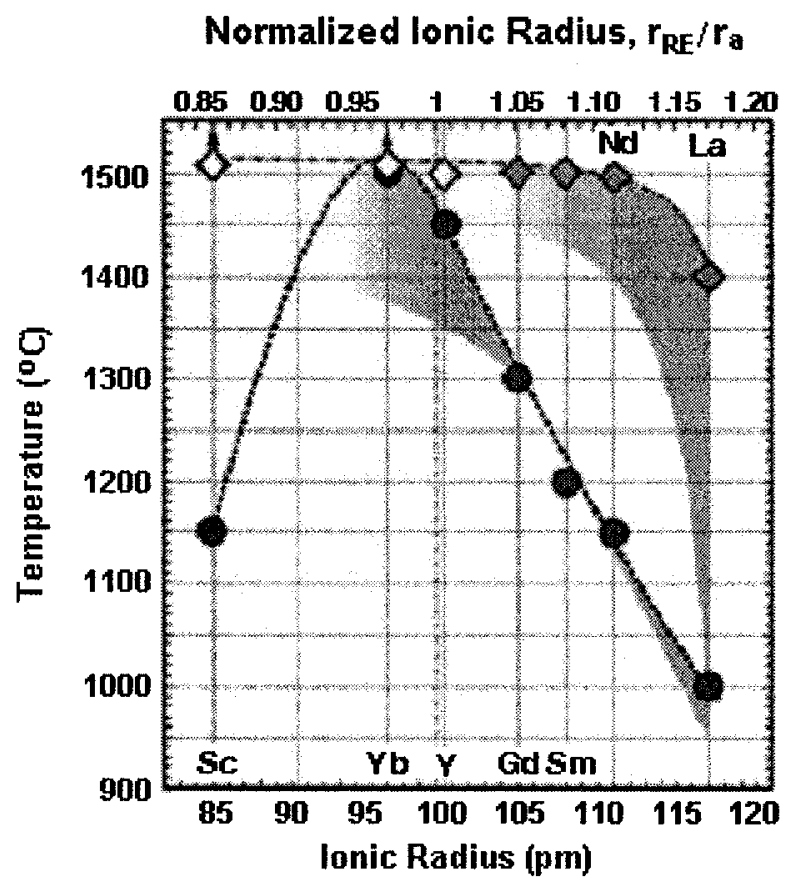

Figure 11 Phase stability of doped binary $\mathrm{ZrO}_{2}-\mathrm{MO}_{1.5}$ compositions (circles) and doped ternary $\mathrm{ZrO}_{2}-\left(\mathrm{Y}_{0.5} \mathrm{M}_{0.5}\right) \mathrm{O}_{1.5}$ compositions (diamonds) [29]. 


\subsubsection{Yttria Stabilized Zirconia}

The addition of metal oxides to zirconia stabilizes the tetragonal $t^{\prime}$ - phase, it also reduces the material's thermal conductivity due to the increase in phonon scattering centers, especially at the vacancy sites. According to thermal conductivity theory, these vacancies strongly scatter phonons by virtue of both missing mass and missing interatomic linkage and therefore result in a decrease in thermal conductivity. While various dopants have been found to reduce the thermal conductivities of zirconia $[1,30$, 31], $\mathrm{Y}_{2} \mathrm{O}_{3}$ stabilized $\mathrm{ZrO}_{2}$ (YSZ) in general is found to be the most suitable one for the TBC applications [29]. The amount of yttria added to zirconia was optimized and it was found that the 7 8 $\mathrm{wt} \%(4 \sim 4.5 \mathrm{~mol} \%)$ yttria partially stabilized zirconia (YPSZ) exhibits the highest degree of resistance to spallation and excellent thermal stability $[32,33]$. The doped zirconia with $4 \sim 5 \mathrm{~mol} \% \mathrm{Y}_{2} \mathrm{O}_{3}$ is able to produce a metastable tetragonal $t$ '- phase when rapidly cooled from high temperature cubic phase region $\left(2200 \sim 2400^{\circ} \mathrm{C}\right.$ or above) to room temperature; this $t^{\prime}$ - phase exhibits high toughness and is non-transformable at temperatures up to $1400^{\circ} \mathrm{C}$. Figure 12 illustrates how the yttria content affects the test lifetime of $\mathrm{Y}_{2} \mathrm{O}_{3}-\mathrm{ZrO}_{2}$ TBC system with differently doped yttria content during thermal cycling tests, where the TBC systems with different yttria content withstood 15001 hour cycles between $990^{\circ} \mathrm{C}-280^{\circ} \mathrm{C}$ [33]. It can be seen that a TBC system with $7 \mathrm{wt} \%$ yttria stabilized zirconia exhibits the maximum test lifetime.

As a comparison, properties of zirconia based ceramics as thermal barrier coating materials and relative properties of TGO, bond coat as well as metal substrate are summarized in Table 2 [35]. 


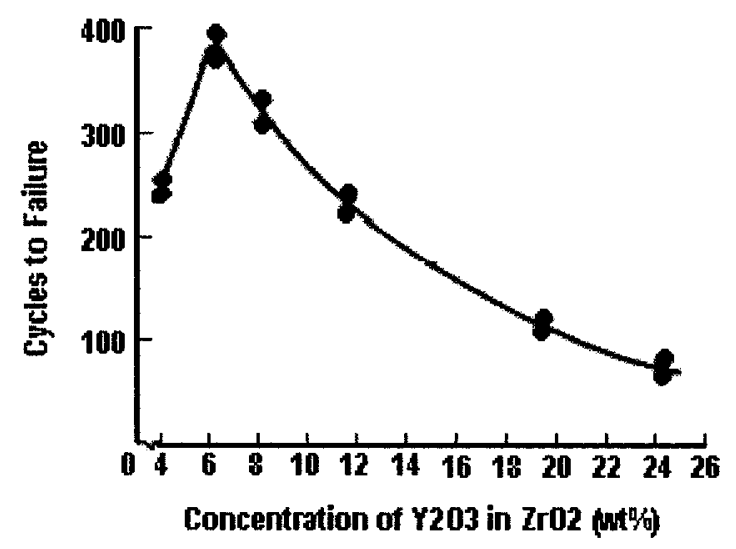

Figure 12 Effects of the yttria content on the lifetime of a $\mathrm{ZrO}_{2}-\mathrm{Y}_{2} \mathrm{O}_{3} / \mathrm{Ni}-16 \mathrm{Cr}-6 \mathrm{Al}-$ $0.2 \mathrm{Y}$ thermal barrier coatings withstood 15001 hour cycles between $990^{\circ} \mathrm{C}-280^{\circ} \mathrm{C} \mathrm{[33]}$.

Table 2 Properties of TBC materials

\begin{tabular}{|c|c|c|c|c|c|}
\hline \multirow[b]{2}{*}{ Materials } & \multicolumn{5}{|c|}{ Properties } \\
\hline & $\begin{array}{l}\text { Melting } \\
\text { point } T m \\
\text { (K) }\end{array}$ & $\begin{array}{l}\text { Thermal } \\
\text { diffusivity } \\
D t h\left(\mathrm{~m}^{2} \mathrm{~s}^{-1}\right)\end{array}$ & $\begin{array}{c}\text { Thermal } \\
\text { conductivity } k \\
\left(\mathrm{~W} \mathrm{~m}^{-1} \mathrm{~K}^{-1}\right)\end{array}$ & $\begin{array}{c}\text { Young's } \\
\text { modulus } E \\
(\mathrm{GPa})\end{array}$ & $\begin{array}{c}\text { Thermal } \\
\text { expansion } \\
\text { coefficient } \alpha\left(\mathrm{K}^{-1}\right)\end{array}$ \\
\hline $\mathrm{ZrO}_{2}$ & 2973 & $0.43 \times 10^{-6}$ & 2.17 & 21 & $15.3 \times 10^{-6}$ \\
\hline $\begin{array}{c}\text { 8YSZ (plasma } \\
\text { sprayed) }\end{array}$ & $\cdots$ & $\ldots$ & 0.8 & 40 & $10.7 \times 10^{-6}$ \\
\hline $\mathrm{Al}_{2} \mathrm{O}_{3}(\mathrm{TGO})$ & 2323 & $0.47 \times 10^{-6}$ & 5.8 & 360 & $8 \times 10^{-6}$ \\
\hline $\begin{array}{l}\text { NiCoCrAIY } \\
\text { (bond coat) }\end{array}$ & --- & --- & -- & 86 & $17.5 \times 10^{-6}$ \\
\hline IN737 & --- & $\cdots$ & --- & 197 & $16 \times 10^{-6}$ \\
\hline
\end{tabular}




\subsection{Thermal Barrier Coating Deposition Technologies}

The performance of TBCs depends not only on the intrinsic properties of TBC materials, but also on the coating's microstructures, which are determined by deposition processes. Since the early application of the calcia- and magnesia-stabilized zirconia TBCs on combustion chamber walls and on burner cans, the air plasma spray (APS) coating technology has been used to deposit both ceramic topcoats and metallic bond coats. Yttria partially stabilized zirconia (YPSZ) was introduced to replace calcia- or magnesiastabilized zirconia in the early eighties to enable further increase in turbine inlet temperature (TIT). In the meantime, the potential application of TBCs extended to highly thermally loaded components like vane platforms and airfoils. Since then, three generations of TBCs have been developed based primarily on different depositing technologies and applications. The first generation was APS MCrAIY bond coat plus APS YPSZ topcoat. However, serious oxidation of bond coat occurred and caused TBC's to fail at elevated temperatures. The low pressure plasma spraying (LPPS) process was therefore adopted for the deposition of MCrAlY bond coat, and the combination of LPPS MCrAIY and APS YPSZ was designated as the second generation of TBC systems. The third generation was the EB-PVD YPSZ ceramic layers with LPPS MCrAlY bond coat. The third generation of TBCs found applications on high-pressure turbine blades and vanes since the first generation TBCs involved plasma spray process which was inadequate due to the poor coating surface finish and cooling hole obstruction problems.

From the historic development of TBCs, it can be seen that the most widely used 
deposition processes for the application of ceramic topcoat are plasma-spraying (PS) and electron beam physical-vapor-deposition (EB-PVD). In this section, the two deposition processes and the associated microstructural characteristics as well as their advantages/disadvantages will be discussed in detail.

\subsubsection{Plasma Spray (PS) Process}

In plasma spraying processes, the powder is injected into a high temperature, high velocity arc plasma so that the powder particles melt. The molten material is accelerated towards the substrate by the carrying gas(es) and impacts on the substrate at a high velocity. The molten droplets striking the substrate freeze while being flattened on the

substrate to form splats. The desired coating is deposited by successive impingement of millions of accelerated heated particles. Since the deposited coatings are mechanically bonded to the substrate, the bond coat should be rough enough so that the top ceramic layer will adhere to it.

Figure 13 shows a schematic of the plasma spray process, where argon or other inert gas is directed into the gun chamber and is ionized by a direct current (DC) electric arc to form plasma gas. This electric arc current, typically 800 to 1000 amps, is created between the anode nozzle and the cathode and can be adjusted to increase the power so that the temperature of the plasma flame can reach the melting temperature of ceramic powder. The powder is injected into the plasma stream at the nozzle and melted in the hottest part of the flame. The powder is accelerated to a high speed of $100-300 \mathrm{~m} / \mathrm{sec}$, striking the substrate where a rapid solidification process occurs. The cooling rate is in 
the order of $10^{6} \mathrm{~K} / \mathrm{sec}$.

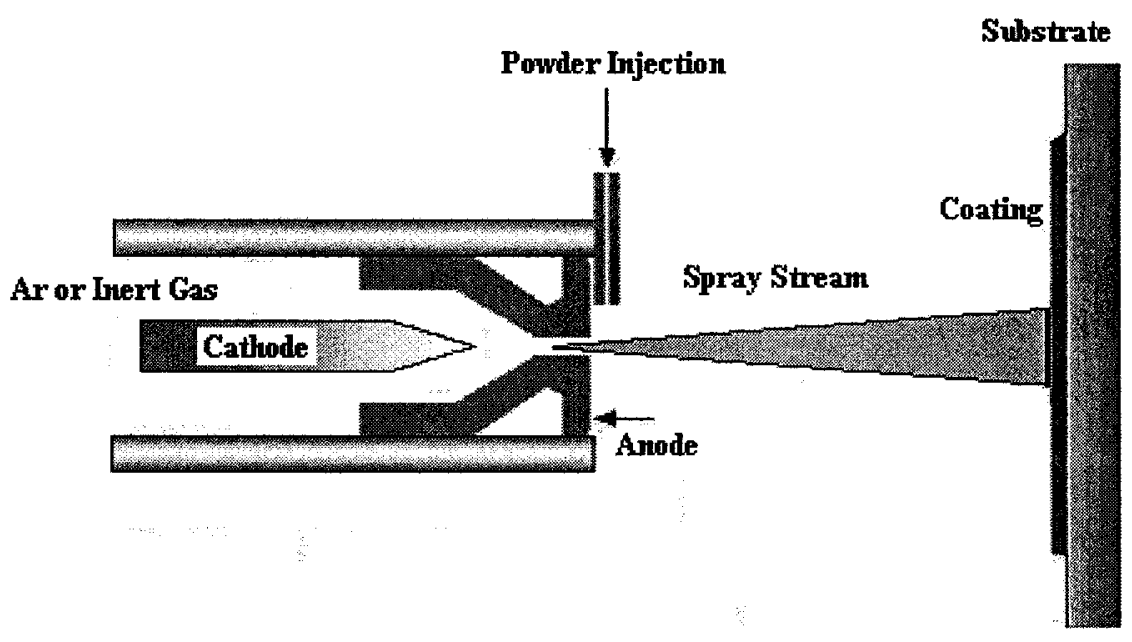

Figure 13 Schematic of the plasma spray process.

For some protective coatings, such as MCrAlY oxidation resistant coatings, a lowpressure plasma chamber is required. Thus the low pressure plasma spraying (LPPS) process was developed, which is a derivative of the air plasma spray (APS) process. APS is much cheaper than LPPS. Both of these processes have been applied to the deposition of ceramic topcoats and metallic bond coats.

\subsubsection{Coating Microstructures Produced by Plasma Spraying Deposition}

The microstructure produced by plasma spraying shows lamellar or flattened splats with microcracks through the splats or inter-splats. These splats lie parallel to the surface of the coating due to the impact of the high speed molten particles on the substrate [5]. The formation of microcracks is caused by the thermal stress arising from the mismatch of thermal expansion coefficients between the coating and substrate, and the rapid cooling rate. Porosities are observed between the splats. The occurrence of porosities can be 
influenced by velocity, powder size and distribution, as well as other plasma spray parameters. For example, the low pressure chamber will result in an increase in the particle velocity, which leads to a stronger impact on the substrate and therefore to a denser, less porous deposit. The rapid solidification of the molten droplets on the substrate could result in the formation of metastable phases, such as tetragonal prime $\left(t^{\prime}\right)$ phase of partially stabilized zirconia, which is especially beneficial for thermal barrier coatings [2].

Figure 14 shows the cross-section of a microstructure of a typical TBC deposited by air plasma spraying. From this figure we can see the network of microcracks (platelet boundaries) and inter-splat pores parallel to the coating surface. The individual platelets formed from the droplets impinging on the surface during the spraying process [36].

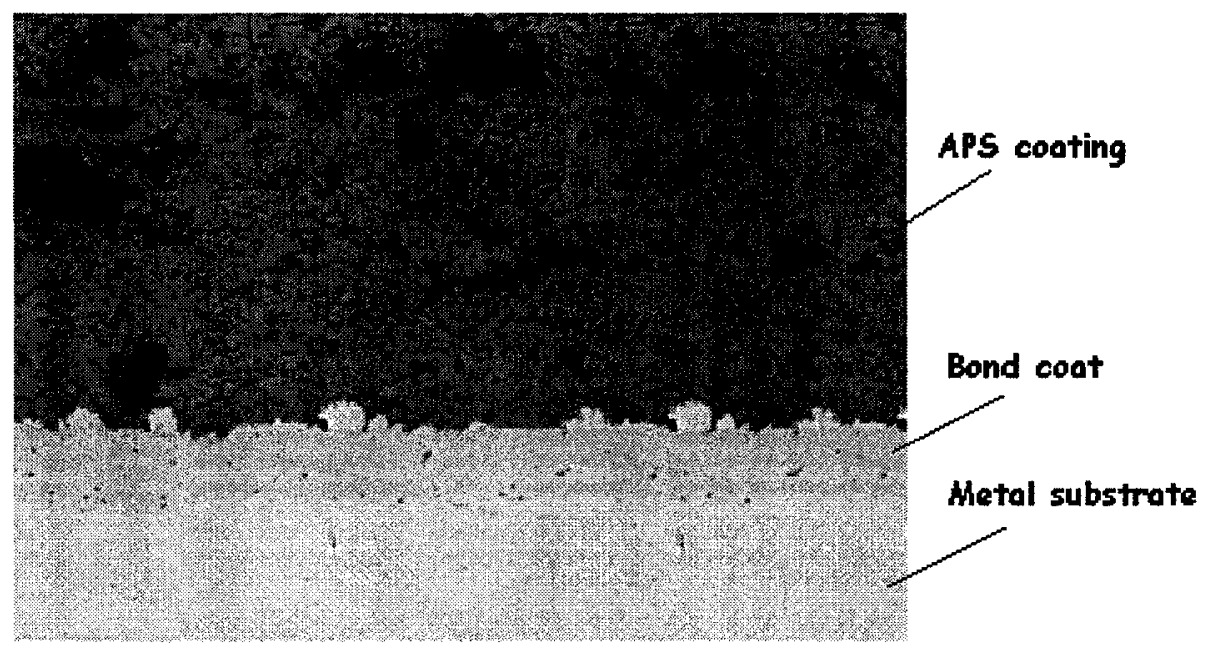

Figure 14 Photomicrograph of an APS TBC showing a laminar structure [36].

The plasma sprayed TBC's, with laminar microstructure, have poor strain compliance and poor erosion resistance, thus limiting their application on high pressure turbine blade 
or nozzle guide vane airfoils where EB-PVD process is preferred.

\subsubsection{Electron Beam Physical Vapor Deposition (EB-PVD) Process}

Physical vapor deposition (PVD) is fundamentally a vaporization coating technique. The basic mechanism is to generate vapor phase from solid stock material. The evaporated material transfers from the source to the substrate, forming a coating. There are various means to generate vapor, such as thermal evaporation, or sputtering process. In EB-PVD, the evaporation is obtained with a focused electron beam. Figure 15 shows a schematic diagram of an electron beam physical vapor deposition (EB-PVD) TBC process, where a focused high-energy electron beam is used to heat and vaporize the ceramic ingots in a vacuum chamber.

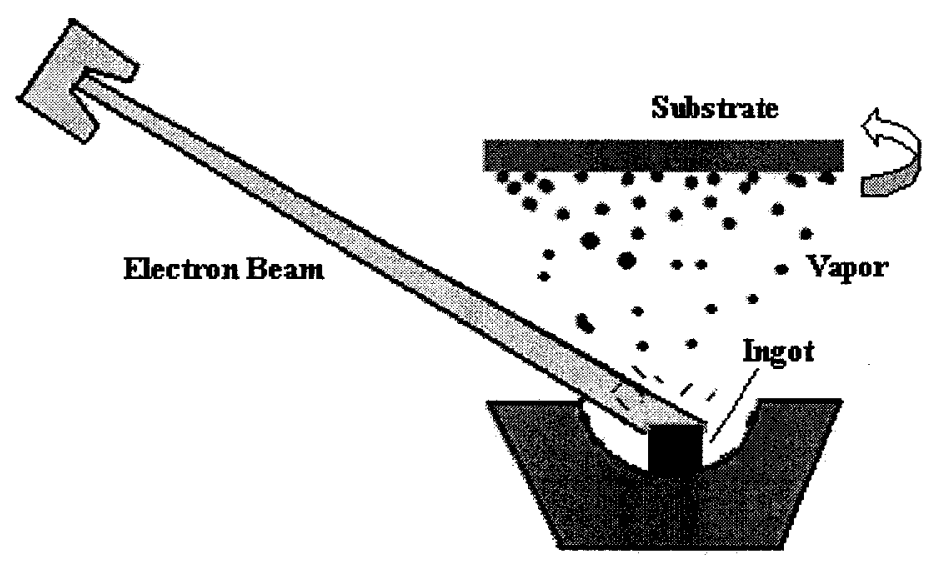

Figure 15 Schematic diagram of the principle of EB-PVD process.

The ingots are bottom fed into the water-cooled crucibles during evaporation to ensure controlled TBC growth. The vapor travels along the line of sight and condenses on the 
rotating substrate surface. The substrate is preheated by an external heating source with the purpose of enhancing the metallurgical bond between the coating and the substrate. When the ceramic is yttria-stabilized zirconia material, a pressure of oxygen is required in order to maintain the stoichiometry of zirconia [37].

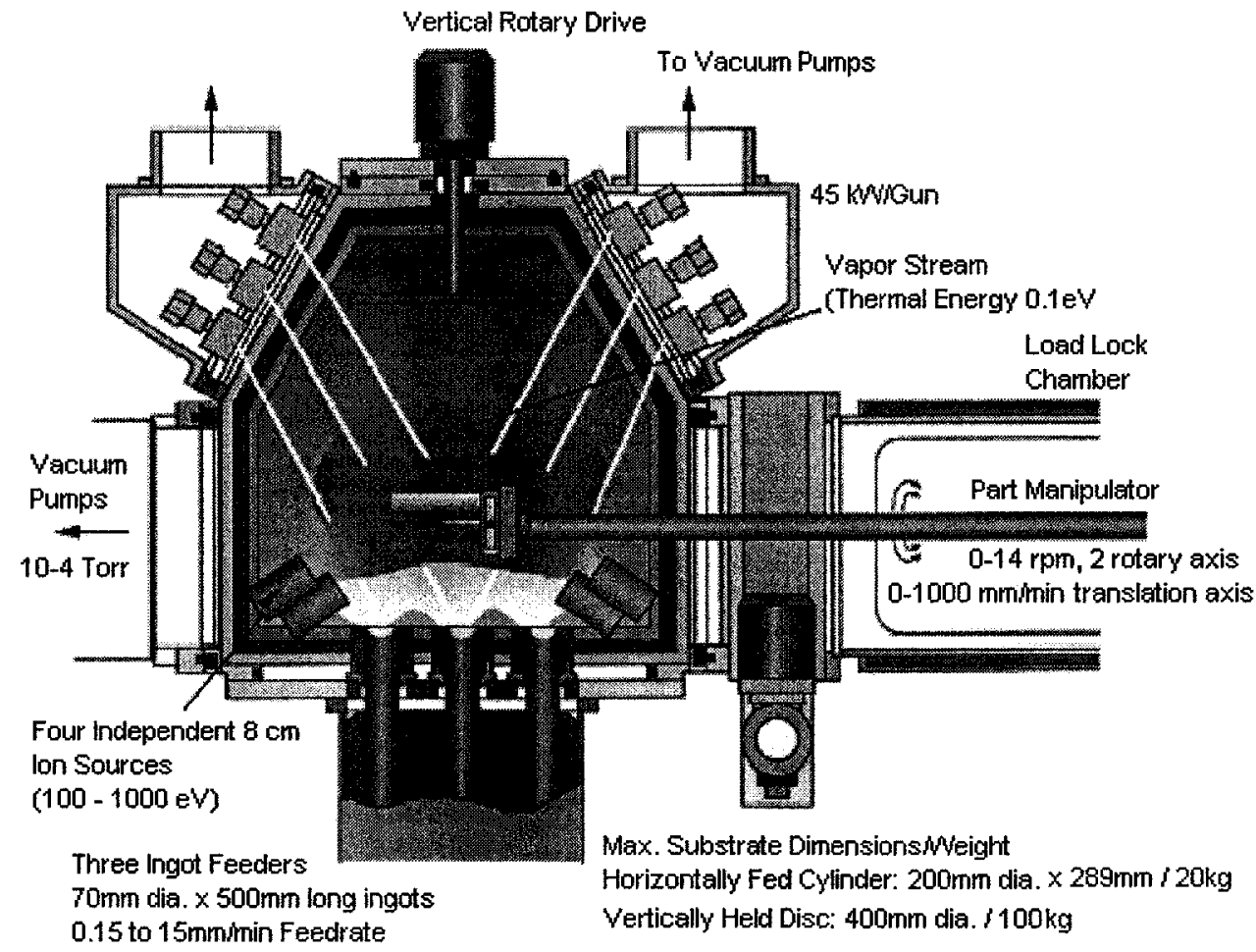

Figure 16 Schematic diagram of EB-PVD unit with 6 EB guns and 3 continuous ingots feeding systems $[37,38]$.

Figure 16 shows a schematic diagram of an EB-PVD unit (ARL Penn State's industrial pilot Sciaky EB-PVD unit) with 6 electron beam (EB) guns at the top of the chamber with the power of $45 \mathrm{~kW} / g u n$, and three continuous ingot-feeding systems at the bottom of the chamber, and a vacuum chamber [38]. Four of the six guns are used to evaporate the coating materials and the other two are used to pre-heat the substrate to enhance the 
adhesion of the coatings. The equipment can offer high deposition rates up to $150 \mu \mathrm{m} /$ minute with an evaporation rate of $10 \sim 15 \mathrm{~kg} /$ hour, compared with other coating deposition techniques, produces dense coatings, and results in low contamination and high thermal efficiency. In addition, coatings produced by EB-PVD have a good surface finish and a uniform microstructure. Using this equipment, the coating compositions can be varied via co-evaporation and coatings comprised of alternating layers of different compositions can be made at relatively low temperatures [38].

\subsubsection{Coating Microstructure Produced by EB-PVD Process}

The dominant characteristics of the TBCs produced by EB-PVD are the columnar microstructure with elongated grains and pores aligned perpendicular to the coating surface, as shown in Figure 17.

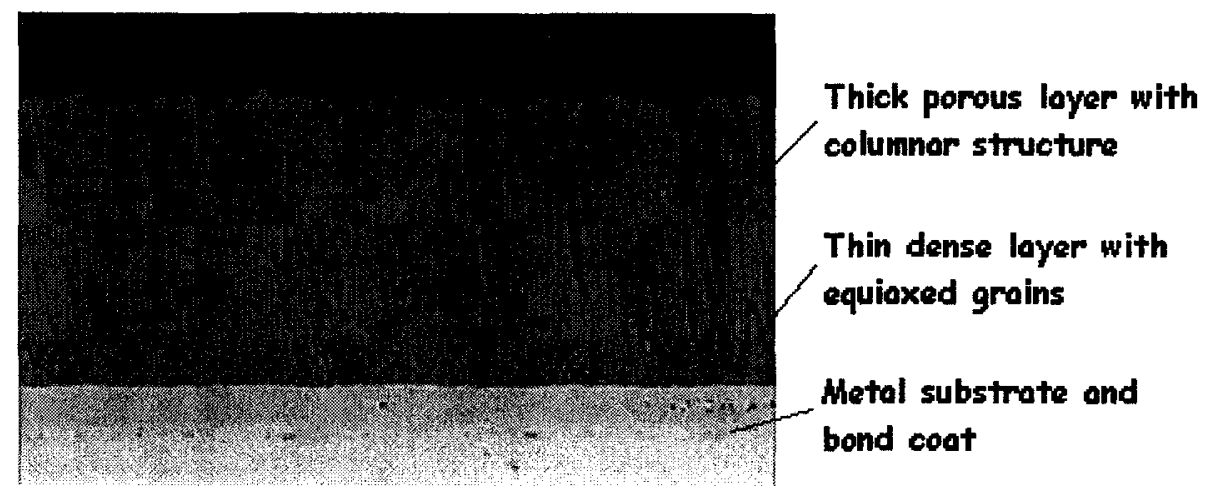

Figure 17 Photomicrograph of a thermal barrier coating (TBC) obtained by electron beam physical vapor deposition (EB-PVD) [37].

The coating shown in Figure 17 can be divided into two parts: a thin dense layer with equiaxed grains and a thick porous layer with columnar structure. Near the interface between the ceramic top layer and the bond coat, a thin dense region of randomly aligned 33 
grains nucleates from the substrate. Further growth of these crystals leads to a columnar texture with pyramidal tips and elongated pores between the columnar grains. This structure results from the evolution of the grain texture, which is controlled by the vapor incidence pattern (VIP), or shadowing mechanism. That is, the growth of grains favors the direction of the incoming vapor. Thus, the grains grow fastest in the direction of vapor incidence, which results in the columnar structure parallel to the vapor beam.

It has been found that the microstructure of the coatings can be affected by variations in deposition conditions, such as substrate temperature, rotational speed, deposition angle or deposition kinetic energy of the vapor [39]. For example, high substrate temperature and high rotational speed can provide parallel columns from the root to the top, which may result in coatings with high density and high hardness. The increase in kinetic energy of the vapor may result in coatings with fewer and smaller voids, smoother surfaces, and higher film density.

\subsubsection{Comparison of Coatings Produced by Plasma Spraying and EB-PVD}

Compared to plasma sprayed coatings, the EB-PVD TBCs have the following advantages:

1. Longer thermal cycling life is attainable due to the better strain tolerance and thus these coatings are suitable for application on parts that are subject to high thermal and mechanical loads. The build-up of any tensile stresses caused by the mismatch of thermal expansion coefficients between the coatings and substrate can be released by the columnar structures with elongated pores between 
columns, and therefore these coatings exhibit very high levels of stress compliance and extended cycling life. A comparison of thermal cyclic lives of air plasma sprayed TBCs and EB-PVD TBCs is shown in Figure 18 [40].

2. The EB-PVD coating generally has lower modulus of elasticity than the counterpart of plasma sprayed coating. For example, the Young's moduli of 8YSZ obtained by sintering, EB-PVD and plasma spraying were measured by laser ultrasonic and resonant techniques. As shown in Figure 19, the modulus of plasma sprayed $8 \mathrm{YSZ}$ is almost the same as that of bulk material at low temperatures, whereas the young's modulus of EB-PVD coatings is less than half that of plasma sprayed coatings [41].

3. The coating surface is smoother, which improves the cooling coefficient of the cooling holes. In an aero engine, the heat transfer coefficient is very sensitive to the friction coefficient of the surface for the high-pressure turbine blade and nozzle guide vane airfoils, thus smoother surface is favorable in terms of improved heat transfer efficiency. The plasma sprayed TBC has a typical surface roughness of $10 \mu \mathrm{m} \mathrm{Ra}$ and a peak to valley height of $80-100 \mu \mathrm{m}$. Whereas EBPVD TBC has a surface finish close to that of the metal surface, typically $1.0 \mu \mathrm{m}$ Ra with a peak to valley height of $10 \mu \mathrm{m}$. The influence of the surface roughness on the primary loss coefficient of the turbine for a high pressure turbine aerofoil was studied [41]. It was found that an increase of $2 \%$ in the primary loss coefficient of the turbine was attributed to the plasma sprayed coating, whereas $1.5 \%$ was attributed to the EB-PVD coating, which was similar to that observed for the uncoated metal surface. 


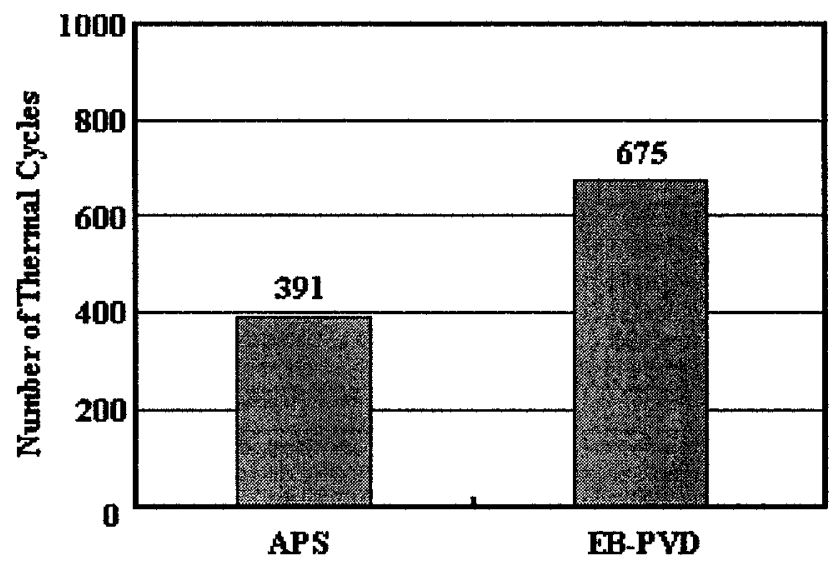

Figure 18 The comparison of thermal cyclic lives of air plasma sprayed TBCs and EB-PVD TBCs [40].

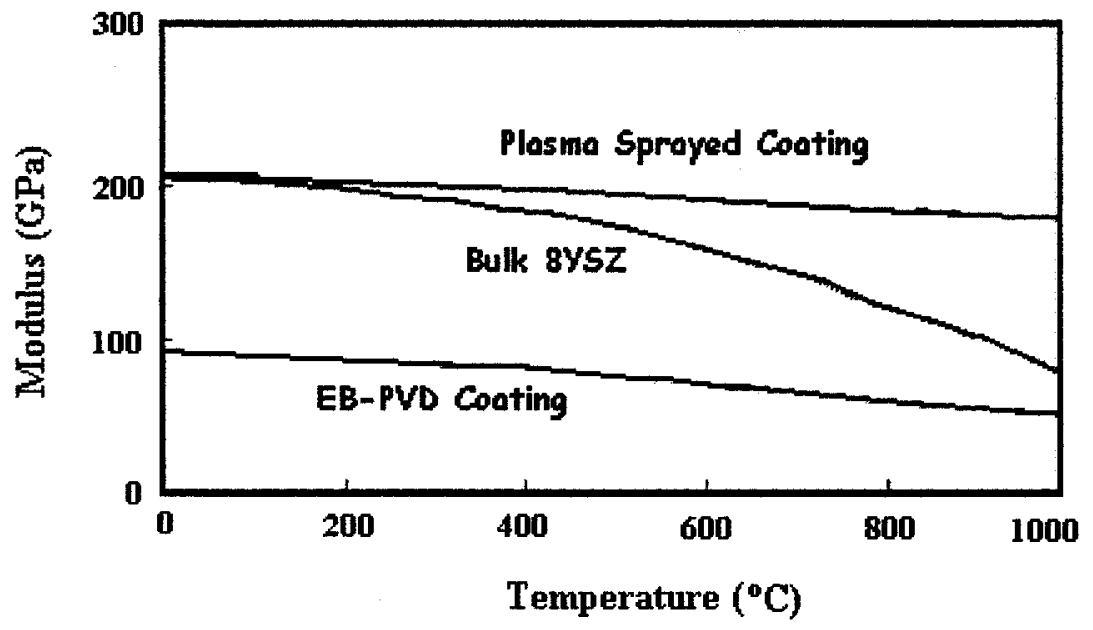

Figure 19 Young's moduli of solid sintered 8wt\% PYSZ, plasma sprayed and EB-PVD 8wt\% PYSZ TBC systems measured by dynamic techniques [41].

4. The columnar structure of the EB-PVD also offers better erosion resistance. Studies showed that the erosion resistance of $\mathrm{TBC}$ is associated with the morphology of the coating microstructures $[42,43]$. Plasma sprayed TBC has a high erosion rate because the near surface material with microcracks parallel to 36 
the surface can spall when the coating is impacted, whereas the columnar structures of EB-PVD coatings do not have cracks parallel to the surface. An erosion test at a temperature of $540^{\circ} \mathrm{C}$ indicated that erosion resistance of $\mathrm{EB}$ PVD TBC was two times that of air plasma sprayed TBC at low angles of attack and ten times more than that of air plasma sprayed TBC at an angle of attack of $90^{\circ}[44]$

5. The EB-PVD technique reduces the likelihood of obstructing the cooling holes. This is due to the deposition mechanism that takes place through the condensation of coating vapor in the EB-PVD process rather than through the impingement of large semi-molten particles in the plasma process [2].

In light of the above comparisons, the EB-PVD process is apparently more advantageous than the traditional plasma spraying process. However, before selecting the EB-PVD process for particular application, the following disadvantages need to be considered.

1. EB-PVD applied TBC has higher thermal conductivity than plasma sprayed TBC. The TBC produced by plasma spraying typically has a thermal conductivity of $0.9 \sim 1.1 \mathrm{~W} / \mathrm{mK}$ at room temperature. This lower thermal conductivity is due to micro-cracks and a high volume fraction of inter-splat pores that are predominantly aligned parallel to the plasma sprayed coating surface, while the EB-PVD TBCs have a thermal conductivity in the range of $1.3 \sim 2.0 \mathrm{~W} / \mathrm{mK}$ [38]. As described earlier, the microstructure of a TBC produced by EB-PVD has two zones: the inner nucleation zone with a large number of interfaces, grain boundaries, microporosity and randomly oriented grains; and the outer zone with 
crystallographically perfect columnar structure and fewer grain boundaries. The inner zone ranges from 1 to $10 \mu \mathrm{m}$ in thickness and exhibits lower thermal conductivity $(\sim 1.0 \mathrm{~W} / \mathrm{m} . \mathrm{K})[45]$. The outer zone has a thermal conductivity value approaching that of bulk zirconia $(2.2 \mathrm{~W} / \mathrm{m} . \mathrm{K})$. Therefore, the apparent thermal conductivity of the EB-PVD coating will be higher than that of the inner part of the coating. The thicker the outer part, the higher the thermal conductivity, which approaches that of bulk zirconia.

2. Less flexible coating composition. The coating composition for PS coatings is determined by the powder composition and can be tailored to any mixture of powder alloys. The EB-PVD process, however, can not always achieve the same coating composition as the ingot due to the differences in vapor pressure of the constituents. When the constituent vapor pressures are close to each other, steady-state vaporization with the proper vapor composition can be achieved. Whereas when the vapor pressures of the alloy constituents differ by significantly larger factors, the required vapor composition cannot be the same as that of the ingot. In addition, the differences in vapor pressure can also result in formation of bubbles and result in inhomogeniety in the coating microstructure. To overcome these limitations, an ion assisted process is required [46]; and in the mean time, the deposition conditions for each new composition have to be optimized. This also results in more complexity and increased cost of the EB-PVD process.

3. The EB-PVD technique is more expensive in both equipment and operation than the plasma spraying technique. As shown in Figure 16, the maximum substrate dimension is limited to $200 \mathrm{~mm}$ in diameter and $289 \mathrm{~mm}$ in length, or the weight is 
limited to $20 \mathrm{~kg}$, the process for EB-PVD is therefore preferred for processing small parts.

To take advantage of EB-PVD coatings and overcome their limitations, particularly the higher thermal conductivity, further research on new TBC materials, and a more cost effective EB-PVD process is required.

\subsection{Failure Mechanisms of TBC Systems}

Thermal cycling tests have shown that TBCs fail in the form of delamination and eventual spallation $[47,48,49,50,51]$. The primary cause of failure is attributed to the thermal expansion mismatch between bond coat and ceramic topcoat. This results in residual stresses developed within the coating system during thermal cycling. Typical thermal expansion coefficients for zirconia based ceramics are about $11 \times 10^{-6} /{ }^{\circ} \mathrm{C}$, the thermally grown oxides (TGO, $\alpha-\mathrm{Al}_{2} \mathrm{O}_{3}$ ) is $8 \sim 9 \times 10^{-6} /{ }^{\circ} \mathrm{C}$, and metallic bond coat is about $13-16 \times 10^{-6} /^{\circ} \mathrm{C}[35]$. When a TBC system is cooled from high operating temperature to room temperature, the thermal expansion misfit between these layers results in very high compression stress in the range of $3 \sim 5 \mathrm{GPa}$ [47]. If this stress exceeds the fracture toughness of any part of the coating, cracking within the coatings occurs. It has been known that this stress is predominately associated with the increased thickness of TGO due to bond coat oxidation. The thermal gradient within the coatings with low thermal conductivities also contributes to the increased stress. In addition, the degradation of the ceramic coating itself due to sintering and aging effects results in changes in thermal and mechanical properties of the coatings and exacerbates the thermal expansion mismatch 
and increases the stress. Failure by hot corrosion is another serious problem when TBCs are applied in diesel engines or gas turbines burning low quality fuel. In this section, the failure modes for different coating structures, the failure mechanisms which govern the TBCs performance as well as the influence of the sintering and aging effects of the coatings at high temperatures on their performance are summarized.

\subsubsection{Failure Mechanisms of Thermal Barrier Coatings}

As mentioned above, the failure of thermal barrier coatings is often associated with bond coat oxidation. Generally, bond coat oxidation has two consequences: the growth of the TGO and aluminum depletion in the bond coat. The growth of the TGO will result in an additional stress, which is the leading cause of the failure of the TBCs. Thus there exists a critical thickness of the TGO, which determines the failure of TBC systems [56]. The aluminum depletion in the bond coat changes the composition of the bond coat, which leads to wrinkles at the TGO/bond coat interface and results in roughening of the bond coat $[52,53]$.

The typical TBC failure mode is buckling and spalling. The buckling can be caused by a small separation at the interface between TGO and bond coat or by cracks between topcoat and TGO. However, these two kinds of failure modes all experience a sequence of crack nucleation, propagation and coalescence events. The coatings produced by either EB-PVD or plasma spraying will have different microstructures, morphologies as well as bonding mechanisms between ceramic topcoat and bond coat, the failure mechanisms are therefore different. 


\subsubsection{Failure mode and mechanisms of plasma sprayed TBCs}

For plasma sprayed TBCs, the surface of the bond coat is roughened to increase the adhesion between ceramics and bond coat. The interfaces between TBC/TGO and TGO/bond coat therefore comprise a large number of undulations with the TGO developed inside the TBC region, as shown in Figure 20.

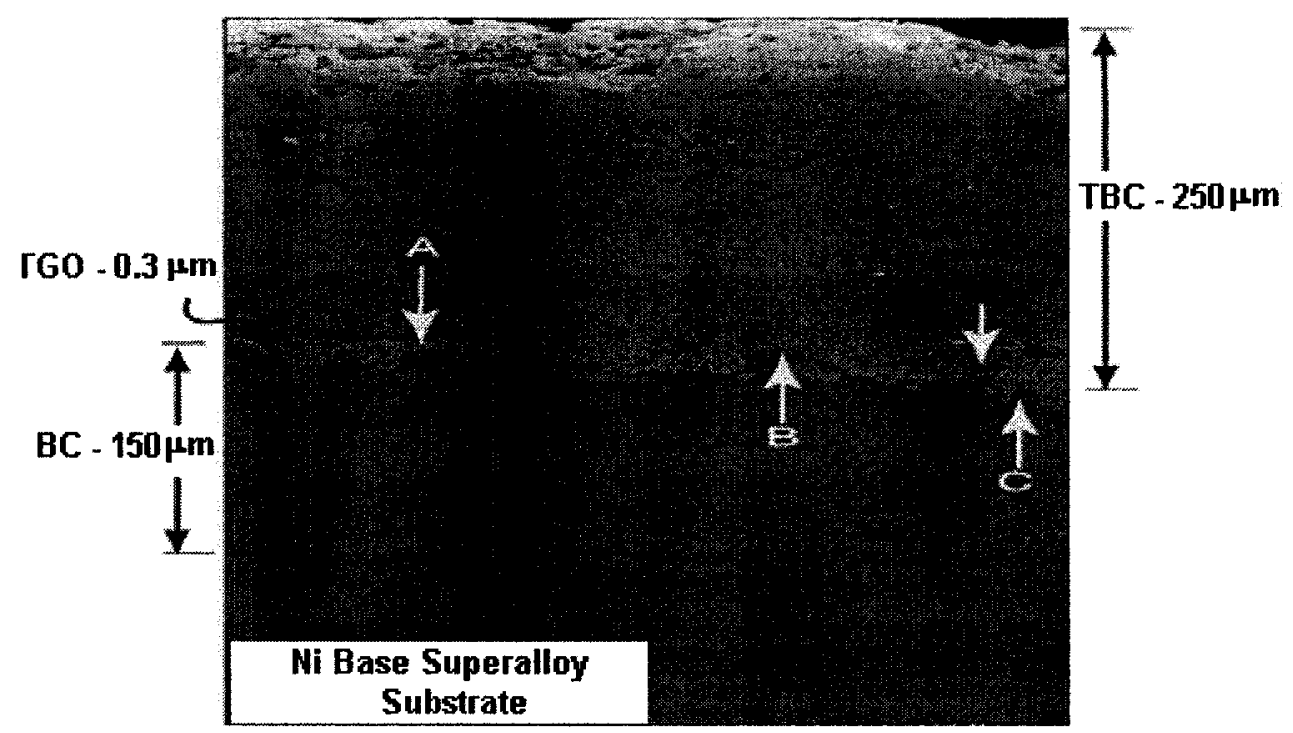

Figure 20 SEM image of a cross section through an as-deposited material revealing the $\mathrm{TBC}$, the TGO and the bond coat. The arrows highlight imperfections around the TBC/TGO interface [56].

Burner rig tests and laser thermal fatigue tests $[48,49]$ showed that when the TBCs are exposed to high temperatures, crack-like separations nucleate around the defects at the interfaces between both the TGO/bond coat and TGO/TBC. These cracks reside within the TBC or TGO, parallel to the interface. In the meantime, the thickness of the TGO increases with exposure time. Upon cooling, the small cracks extend laterally from the 41 
imperfections. The coalescence of the cracks in the TBC or through TGO causes either a large-scale buckle or an edge delamination that results in the spalls of the coatings, as shown in Figure 21 [55].

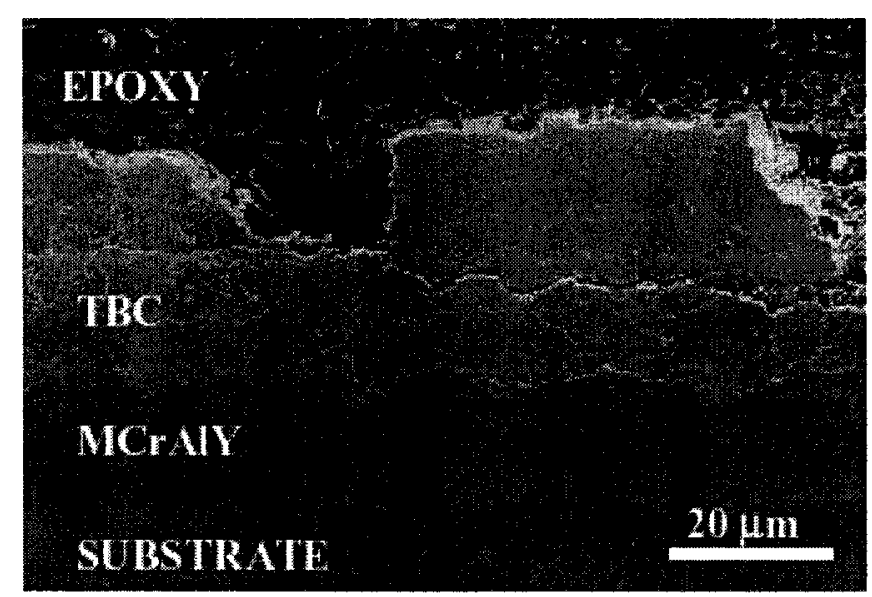

Figure 21 SEM images of delaminations introduced by an as -deposited plasma sprayed coating [55].

The failure mechanism due to the bond coat oxidation for the PS coatings has been studied and well explained using scaling laws [56]. When the PS TBCs are exposed to high temperatures, the thickness of TGO increases due to the bond coat oxidation. Therefore the tensile stresses form as a result of the thermal expansion misfit. Adjacent to the imperfections at the interfaces between $\mathrm{TBC} / \mathrm{TGO}$ or $\mathrm{TGO} /$ bond coat, these stresses are normal to the interface and therefore result in radial cracks in the TBC, as shown in Figure 22 (a). During thermal cycling, additional radial cracks will be developed and eventually penetrate TGO until the cracks coalesce at the imperfection interface, as shown in Figure 22 (b). 


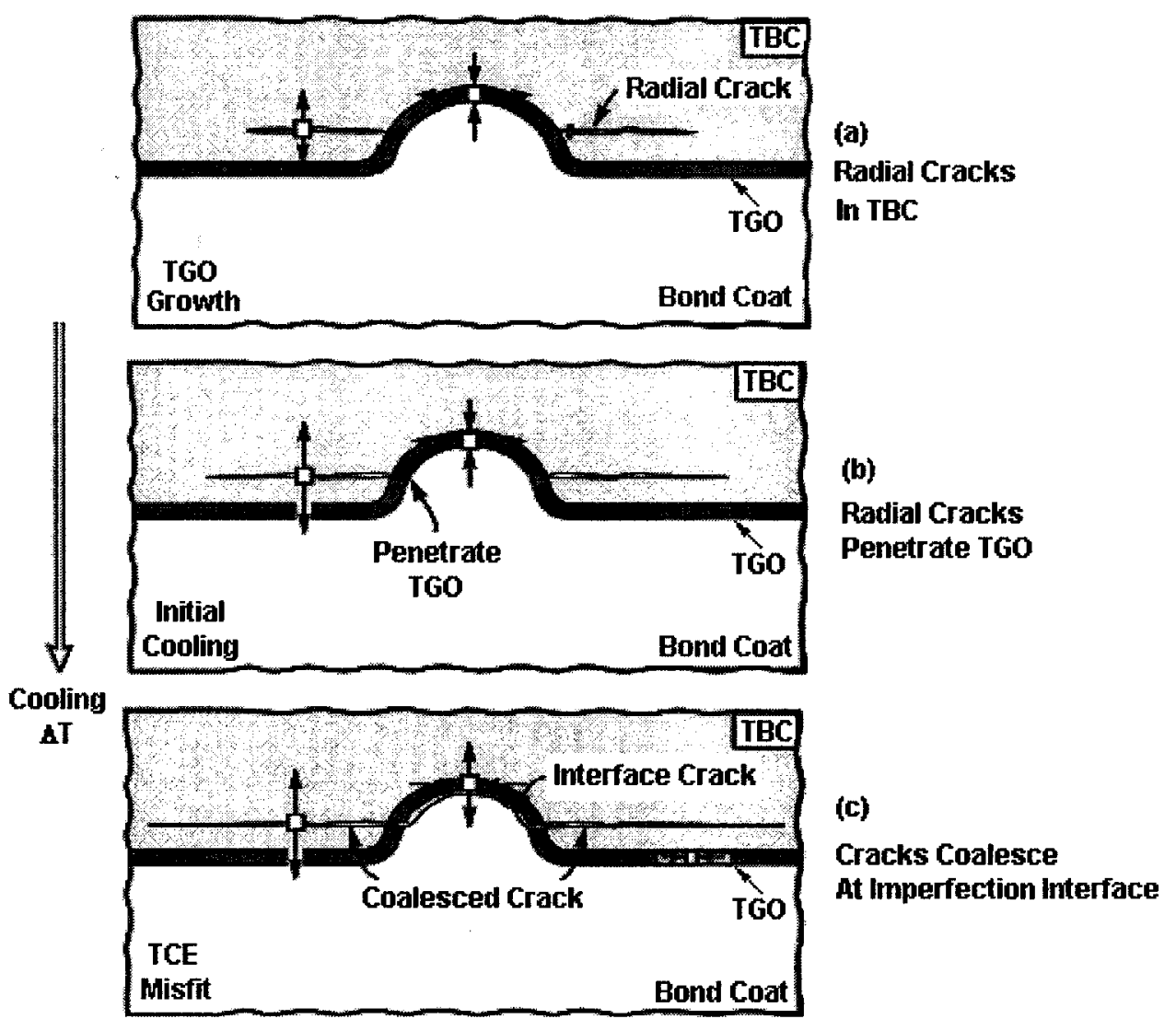

Figure 22 Schematic illustrations of the failure mechanisms for Plasma Sprayed TBCs $[56]$.

\subsubsection{Failure mode of EB-PVD coatings}

Unlike the failure of PS TBCs, where the cracking propagation and spalls occur in the TBC or TGO near the bond coat interface due to the roughened bond coat surface; the EB-PVD TBCs failed by cracking at the interface between TGO and bond coat due to the separation at $\mathrm{TGO} /$ bond coat interface.

Figure 23 shows the sequence of failure of an EB-PVD TBC; the steps are the interface separation, buckling, buckling propagation and spalling of TGO away from the substrate. 
At high temperatures, interface separation happens due to the tensile stress developed within the TGO layer. When the EB-PVD TBCs are exposed to high temperatures for a long time, the thickness of the TGO will be increased, accompanied by compressive stresses developed on cooling. With more thermal cycling, the continued buckling results in the final spalling of the coatings and failure occurs.

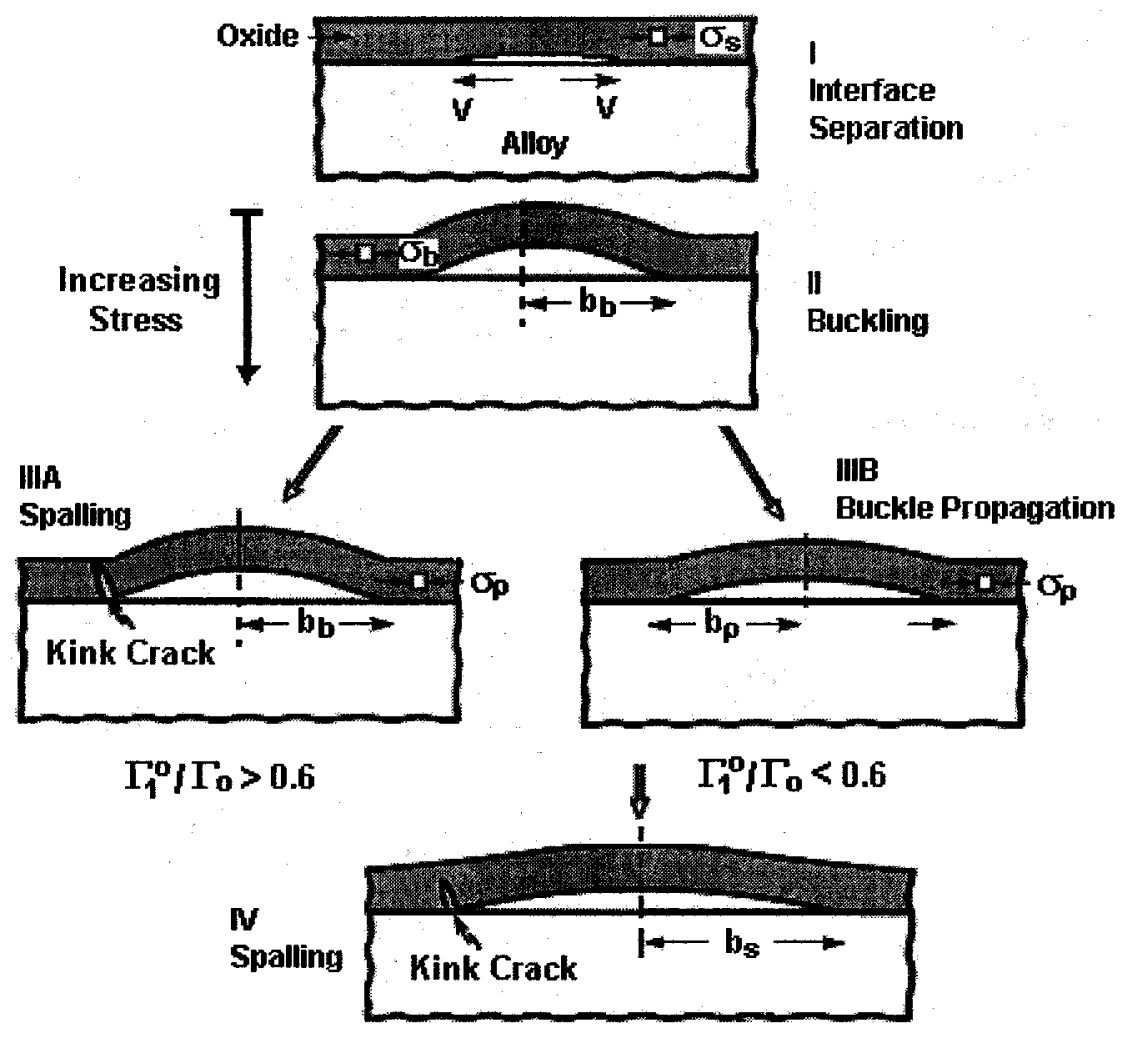

Figure 23 Schematic indicating the sequence of events when a compressed film buckles and spalls away from a substrate $[52,53]$.

Figure 24 shows the interface separation after 180 thermal cycles [56]. The initial interface separations for EB-PVD TBCs have been explained by Clarke $[7,52,53]$ using two mechanisms: one is the "ratcheting" mechanism, which is motivated by the lateral compressive stress; another is the surface displacement associated with volumetric 
changes in the bond coat as aluminum depletion occurs.

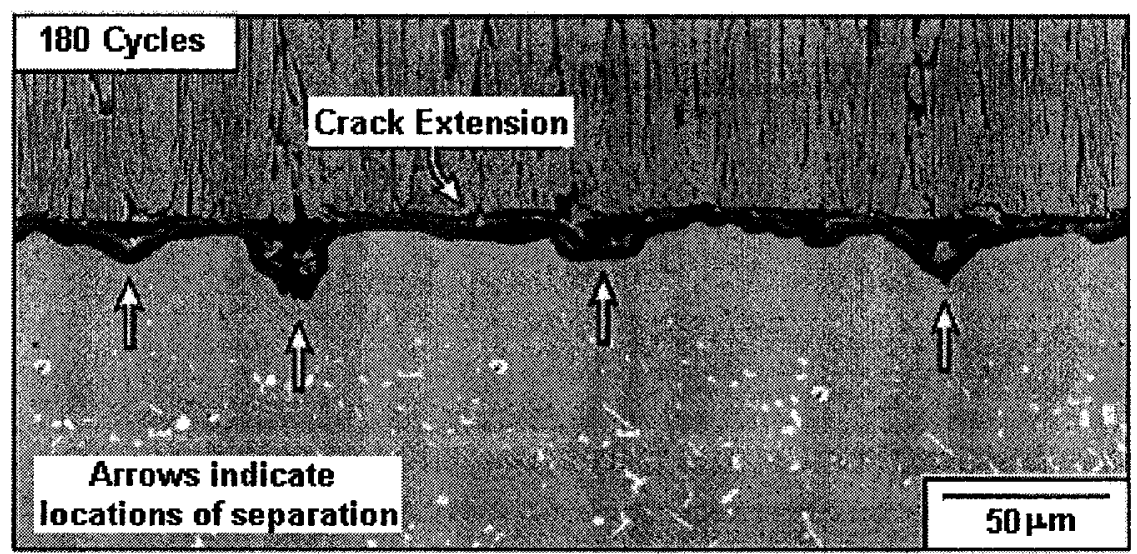

Figure 24 Interface separations for the EB-PVD TBCs after 180 thermal cycles [56].

\subsubsection{Hot Corrosion Failure}

When TBCs are used in diesel engines, another major degradation mechanism is the corrosive attack due to the low quality fuels with high amounts of impurities including V, $\mathrm{Na}$ and $\mathrm{S}$.

During combustion, these fuel impurities are oxidized to form oxides with strong acid and base properties forming salts such as $\mathrm{Na}_{3} \mathrm{VO}_{4}\left(3 \mathrm{Na}_{2} \mathrm{O} . \mathrm{V}_{2} \mathrm{O}_{5}\right), \mathrm{NaVO}_{3}\left(3 \mathrm{Na}_{2} \mathrm{O} . \mathrm{V}_{2} \mathrm{O}_{5}\right)$ and $\mathrm{V}_{2} \mathrm{O}_{5}$. At high operating temperatures, the reaction between oxide and $\mathrm{YSZ}$ will extract $\mathrm{Y}_{2} \mathrm{O}_{3}$ stabilizer from inside the coatings and result in a destabilization of the tetragonal phase. This leads to a detrimental phase transformation of $t$ ' phase discomposed into $t$-phase and $m$-phase. In addition, the porous structure of TBC allows the impurities to penetrate easily into the coating and exacerbate the corrosion failure. Thus, to protect TBCs from failure due to hot corrosion, materials with corrosion resistance are required. 
The hot corrosion behavior of TBCs has been studied by Mifune et al [57]. Two types of oxide ceramics $\mathrm{C}_{2} \mathrm{~S}-15 \mathrm{CZ}\left(2 \mathrm{CaO} . \mathrm{SiO}_{2}-15 \mathrm{wt} \% \mathrm{CaO} . \mathrm{ZrO}_{2}\right)$ and $8 \mathrm{YSZ}$ were investigated in hot corrosion testing environments with $\mathrm{V}_{2} \mathrm{O}_{5}-\mathrm{Na}_{2} \mathrm{SO}_{5}$ corrosive ash for 3 hours at $1000^{\circ} \mathrm{C}$. It was found that the $8 \mathrm{YSZ}$ coating partially spalls due to the reaction of $\mathrm{Y}_{2} \mathrm{O}_{3}$ with $\mathrm{V}_{2} \mathrm{O}_{5}$, which resulted in the loss of its function. On the other hand, the $\mathrm{C}_{2} \mathrm{~S}-15 \mathrm{CZ}$ coating showed adequate resistance to hot corrosion. It has been found that the $\mathrm{Sc}_{2} \mathrm{O}_{3}$ and $\mathrm{Y}_{2} \mathrm{O}_{3}$ co-doped $\mathrm{ZrO}_{2}$ (SYSZ) had resistance to destabilization by molten $\mathrm{NaVO}_{3}$ at $700^{\circ} \mathrm{C}$ and $900^{\circ} \mathrm{C}[58]$; where the $\mathrm{SYSZ}$ powders stabilized by $\mathrm{Sc}_{2} \mathrm{O}_{3}$ plus $10 \mathrm{~mol} \%$ $20 \mathrm{~mol} \% \mathrm{Y}_{2} \mathrm{O}_{3}$, after $1400^{\circ} \mathrm{C}$, showed 5-10 times better resistance to vanadate-induced destabilization than the only $\mathrm{Y}_{2} \mathrm{O}_{3}$ stabilized $\mathrm{YSZ}$ powder. In the selection of alterntive doping oxides for zirconia, these materials should be kept in mind.

\subsubsection{Degradation of Ceramic Topcoats}

The failure mechanisms for TBCs produced by either PS or EB-PVD all suggest that coating delamination is caused by the increased stresses within the coatings. This stress is primarily induced by bond coat oxidation. However, it has been found that the degradation of the TBC itself also contributes to the increases in coating stresses and therefore limits the durability of the TBC system. The degradation of the TBC may result from the sintering and densification of the ceramic coatings, or phase decomposition for zirconia based ceramics. During high temperature cyclic exposure, any of these forms of degradation will produce additional stresses within the coatings, as described below. 


\subsubsection{Sintering and densification of ceramic coatings}

A high porosity level within ceramic topcoat of TBCs, typically $\sim 12-15 \%$ for PS coatings [59] and 20\% for EB-PVD coatings [60], can reduce density, Young's modulus and thermal conductivity. In particular, the existence of porosity can offer better strain tolerance, and the ability to accommodate thermal expansion mismatch between the ceramics and the metallic bond coat and substrate. However, when the coatings are exposed to high temperatures for extended times, porosity will decrease and the coatings will be densified to reduce the surface energy. This phenomenon is called sintering and has been observed for both PS coatings and EB-PVD coatings. The sintering and densification of the coatings will lead to an increase in Young's modulus and thermal conductivity, and strain energy in the TBCs $[59,60]$.
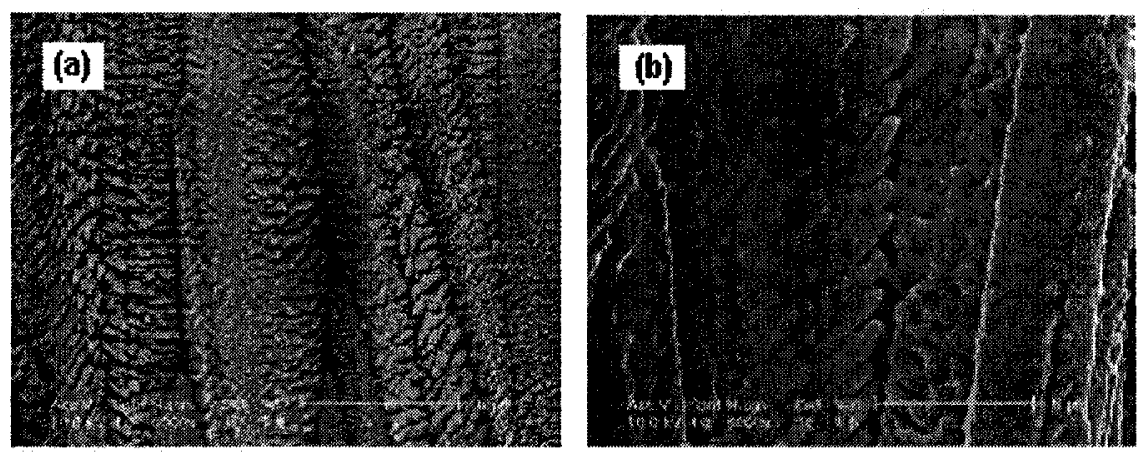

Figure 25 Cross-sectional images showing the microstructural evolution of EB-PVD coating with different temperatures and times (a) as-deposited and (b) after heat treatment at $1200^{\circ} \mathrm{C}$ for 120 hours [62].

The sintering was observed in the EB-PVD deposited TBCs on annealing, where the columnar structures became concave shaped due to stress relief in the root area or/and 
enhanced sintering at the upper side $[61,62,62]$. The microstructure evolution of EBPVD coatings after isothermal treatment is shown in Figure 25. In the as-deposited state, the microstructure exhibits columns with a feathery shape. After heat treatment, the feathery morphology of the individual columns gradually disappears and changes into a smooth fluctuating surface with necks adjacent to columns. The sintering of TBC was found to be controlled by the driving force [61], which depends on the coating microstructure features and substrate constraint. Thus the difference in microstructure of TBC across its thickness leads to different degree of sintering. It has been found that the smaller columns near the interface are easier to sinter compared with the larger columns near the top surface, and also the sintering is constrained by metal substrate while the coating surface is less constrained [62].

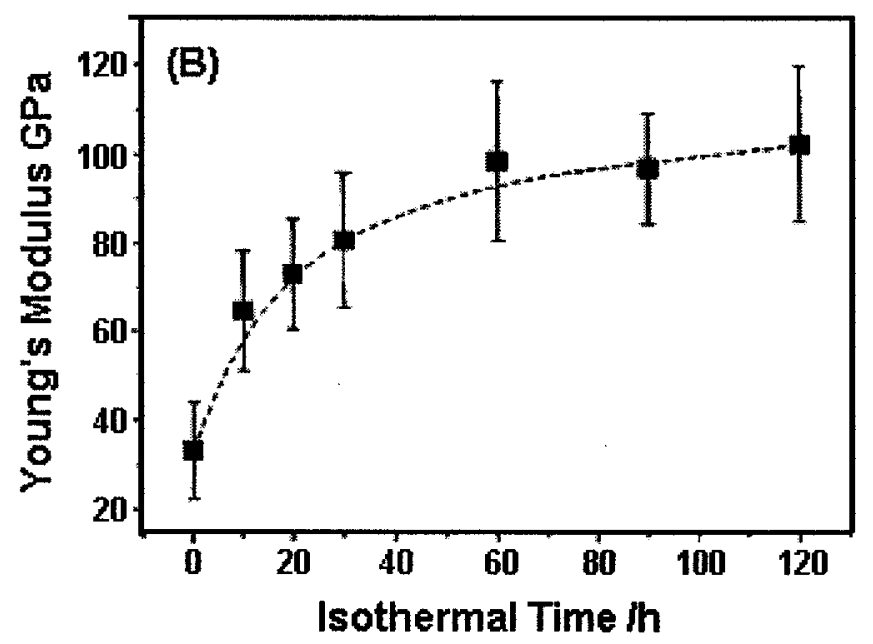

Figure 26 Young' modulus of EB-PVD coating increases with time at $1200^{\circ} \mathrm{C}$ [62].

The effects of densification of the coatings on Young's modulus and residual stresses were examined and the results are given in Figure 26 and Figure 27 [62]. These figures 
indicate that with increased degree of sintering, the strain energy density inside the coatings increases. Thus the sintering and densification effects may exacerbate the delamination of the coatings and further limit the life of TBCs.

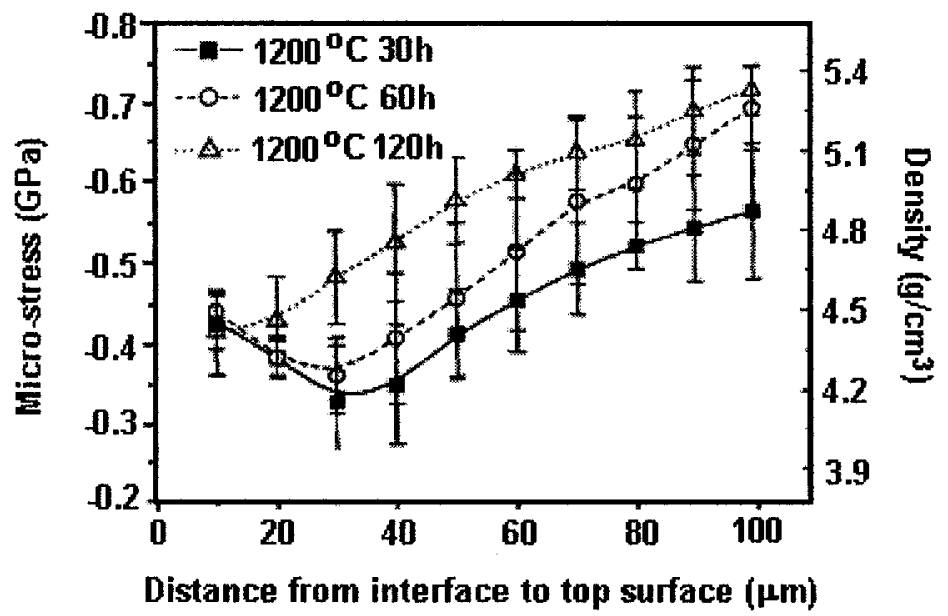

Figure 27 Micro-thermal stress and density as a function of distance from the interface to top surface of free-standing EB-PVD coating with different times [62].

\subsubsection{Aging and phase decomposition}

When zirconia alloyed with $4 \sim 5 \mathrm{~mol} \% \mathrm{Y}_{2} \mathrm{O}_{3}$ is rapidly cooled from high temperature cubic phase region $\left(2200 \sim 2400^{\circ} \mathrm{C}\right.$ or above) to room temperature, a metastable tetragonal phase termed the $t^{\prime}$ phase is produced by a diffusionless process. This quenched $t^{\prime}$ phase is crystallographically the same as $t$ phase, i.e., the same unit cell arrangement and a lower c/a ratio; but morphologically different. However, the microstructure features such as antiphase boundaries, twins and domains are different [17]. Coating deposition processes, such as plasma spraying or EB-PVD, are very similar to the rapid quenching process. Thus the tetragonal $t$ ' phase will form and will 
not transform to other phases at normal operating temperatures.

At temperatures above $1200^{\circ} \mathrm{C}$, the yttria partially stabilized zirconia based coatings are unable to provide the intended thermal protection to metallic substrates. The tetragonal $t^{\prime}$ phase decomposes to yttria-poor tetragonal and yttria-rich cubic phases on extended exposure at temperatures above $1200^{\circ} \mathrm{C}$. On cooling, the tetragonal phase will transform into monoclinic phase, which results in the coating cracks $[63,64,65,66,67]$. The experiment conducted by $\mathrm{Li}$ et al. [65] indicated that the lattice parameter ratio c/a of $t$, phase increases with thermal cycling at $1050^{\circ} \mathrm{C}$, corresponding to a decrease in the content of $\mathrm{Y}_{2} \mathrm{O}_{3}$ of the $t^{\prime}$ phase. That means that the nonequilibrium $t^{\prime}$ phase slowly decomposed into tetragonal phase with low $\mathrm{Y}_{2} \mathrm{O}_{3}$ content and cubic phase with high $\mathrm{Y}_{2} \mathrm{O}_{3}$ content. After 300 cycles, small amounts of monoclinic phase were observed, which resulted from the tetragonal to monoclinic phase transformation during cooling.

\subsection{Heat Transfer through Ceramic Materials}

In gas turbine hot sections, the surface of the components is heated by the hot gases by means of thermal convection and thermal radiation and therefore results in an increased surface temperature. Inside the solid components, the heat can be transferred by thermal conduction or thermal radiation. When zirconia based ceramics, as thermal barrier coatings, are applied to the combustor liners, turbine vanes and turbine blades, the heat flow is retarded due to their insulation to thermal conduction and the temperatures on the superalloy substrate are thus reduced. However, zirconia based ceramics is transparent or partially transparent to a certain wavelength range of thermal radiation. Thus, some 
wavelength range of the radiation may transmit into the coatings and through the coatings to the metal substrate. Generally speaking, heat transfer in ceramic coatings is governed by two mechanisms. The first is thermal conduction, which transfers heat from hot coating surface to the metal substrate, depending on the thermal conductivities of the coating materials. The coating surface is heated by the convection of hot gases and the absorbed radiation when the radiation wavelength is beyond the transparent range of the coating. The second type is thermal radiation falling within the transparent and partially transparent range of ceramics. It includes internal radiation emitted by hot coatings and external radiation emitted by hot gases and transmitted into the coatings. Radiation within transparent wavelength range of ceramic coatings will directly transport through the coatings to the metal substrate without any absorption, and the rest of the radiation will experience absorption and re-emission by hot coatings when it passes through the TBCs. In the following sections, both heat transfer mechanisms will be thoroughly discussed.

\subsubsection{Thermal Conduction in Ceramic Materials}

In a ceramic material, thermal energy is conducted by means of lattice vibration. In a regular lattice with harmonic forces between atoms, if all the atoms vibrate with the same frequency $\omega_{k}$, it is called the normal vibration of the lattice, where the subscript index $k$ represents the wave vector. On the scale of the lattice spacing, the wave vector $k$ is discrete. For example, in a one-dimensional monatomic chain with $G$ identical ions of mass $M, k$ can only have values which meet the following condition: 


$$
k=\frac{l}{G} \frac{2 \pi}{a}
$$

where $l$ is an arbitrary integer, and $l=1,2, \cdots, G$; and $a$ is the distance between adjacent atoms. The vibration frequency $\omega_{k}$ is therefore determined by:

$\omega_{k}=\omega_{\max }\left|\sin \frac{k a}{2}\right|$

where $\omega_{\max }$ is the maximum frequency, depending on the bonding force. (Eq. 7) shows that each atom may oscillate in $N$ different normal modes, with normal frequencies $\omega_{k}$ corresponding to the allowed $k$ values, which are provided by (Eq. 6).

These quantized vibration modes are called phonons, an analog of photon in an electromagnetic wave. In a mode of frequency $\omega_{k}$, each phonon carries the energy of $\hbar \omega_{k}$ and propagates with the phonon group velocity $v_{G k}=d \omega_{k} / d k$, where $\hbar$ is Planck's constant. When phonons travel in one direction, the heat current carried by them can be expressed by the product of their thermal energy and the group velocity of propagation $[68,69]:$

$h=\sum_{k} N_{k} \hbar \omega_{k} v_{G k}$

where $N_{k}$ is the phonon distribution number at the mode $k$ departing from the thermal equilibrium distribution. 
If the potential energy of an atom in a perfect crystal were exactly quadratic without the anharmonic terms in the displacement from equilibrium, the lattice waves would propagate without interacting with one another. The heat would be conducted without any thermal resistance. That means that the phonon thermal conductivity would be infinite, even in the absence of temperature gradient. However, in a real crystal, the phonons will be scattered due to the existence of anharmonicities in the atomic interactions and the crystal imperfections. And the phonon transport is therefore retarded, resulting in a finite thermal conductivity. The phonon transport involving various scattering processes can be described using the Boltzmann transport equation [70]:

$-v_{G k} \cdot \nabla T \cdot \frac{\partial N_{k}}{\partial T}+\left(\frac{\partial N_{k}}{\partial t}\right)_{\text {scattering }}=0$

To solve (Eq. 9), a relaxation time $\tau$ was assigned to each phonon mode. It is assumed that scattering processes tend to restore a phonon distribution to the thermal equilibrium distribution at a rate proportional to the departure of the distribution from equilibrium, so that:

$\left(\frac{\partial N_{k}}{\partial t}\right)_{s c a t t .}=\frac{N^{0}-N_{k}}{\tau}$

where $N^{0}$ is the phonon distribution number with energy of $\hbar \omega_{k}$ at temperature $T$ in thermal equilibrium, given by the Bose-Einstein Distribution: 


$$
N^{0}=\frac{3}{\exp \left(\hbar \omega_{k} / k_{B} T\right)-1}
$$

where $k_{B}$ is the Boltzmann constant. The number 3 in (Eq. 11) means that a phonon has three possible polarization states (one longitudinal and two transverse) which have the same energy.

(Eq. 9) and (Eq. 10) can be used to solve (Eq. 8) for $N_{k}$. By integrating over all phonon modes, assuming an isotropic phonon group velocity, and combining the Debye model, the thermal conductivity $\lambda$ can be expressed as:

$\lambda=\frac{1}{3} v_{G}^{2} \int^{\theta / T} \tau(x) c(x) d x$

where $x=\hbar \omega / k_{B} T ; \theta$ is Debye temperature and has the form of $\theta=\hbar \omega_{D} / k_{B}$; $\omega_{D}=\left(6 \pi^{2} G / V\right)^{1 / 3} v_{G}$, where $V$ is the volume of the crystal; and $c(x)$ is the specific heat capacity, having the form:

$$
c(x)=\frac{3 k_{B}}{2 \pi^{2} v_{G}^{3}}\left(\frac{k_{B}}{\hbar}\right)^{3} T^{3} \frac{x^{4} e^{x}}{\left(e^{x}-1\right)^{2}}
$$

The relaxation time can be expressed as the ratio of a mean free path to a velocity, so that the conductivity may also be written as:

$$
\lambda=\frac{1}{3} v_{G} \int l(x) c(x) d x
$$


where $l(x)=v_{\mathrm{G}} \tau(x)$ is defined as the phonon mean free path.

The above equation indicates that the evaluation of thermal conductivity needs knowledge of the phonon group velocity, the specific heat and the phonon mean free path or relaxation time. Generally, phonon group velocity is the speed of sound. At temperatures higher than the Debye temperature $(T>\theta)$, the specific heat approaches the classical value of $3 k_{B}$. Thus, at high temperatures the thermal conductivity of a crystal is determined by only the value of phonon mean free path or relaxation time. The phonon mean free path is limited by various scattering processes and interactions of phonons, having the form $[71,72]$ :

$\frac{1}{l(\omega, T)}=\sum_{i} \frac{1}{l_{i}(\omega, T)}$

(Eq. 15)

where the suffix $i$ denotes various processes, including the interactions of phonons with each other due to the anharmonic forces between the atoms, and static imperfections of crystals such as point defects or scattering by boundaries. The phonon mean free path caused by these processes may be estimated by perturbation theory which is further described in the next section.

\subsubsection{Intrinsic thermal conductivity at high temperatures}

In a real crystal, the vibrations of individual atoms are not independent but correlated since the atom is not bound to a fixed point in space but bound to its neighbor atoms such that the equilibrium site depends on the instantaneous positions of the neighboring atoms 
[72]. Thus, the forces between atoms are no longer harmonic, and a small anharmonic component is added to the lattice forces, which results in the interaction of phonons with each other and further limits the values of the phonon mean free path.

The phonon interactions can be described by the anharmonic term $H^{\prime}$ in the potential energy $[71,72]$ as a perturbation Hamiltonian:

$$
H^{\prime}=\sum_{k_{1}, k_{2}, k_{3}} c_{3}\left(\bar{k}_{1}, \bar{k}_{2}, \bar{k}_{3}\right) \xi^{*}\left(\bar{k}_{3}\right) \xi\left(\bar{k}_{1}\right) \xi\left(\bar{k}_{2}\right)
$$

where $\xi^{*}\left(\bar{k}_{3}\right) \xi\left(\bar{k}_{1}\right) \xi\left(\bar{k}_{2}\right)$ means that a phonon $\bar{k}_{3}$ is created at the same time phonons $\bar{k}_{1}$ and $\bar{k}_{2}$ are destroyed due to the interaction. The energies from phonons $\bar{k}_{1}$ and $\bar{k}_{2}$ are transferred into the phonon $\bar{k}_{3}$.

The coefficient $c_{3}\left(\bar{k}_{1}, \bar{k}_{2}, \bar{k}_{3}\right)$ depends on the nature of the anharmonic forces and is very important for the interactions of different modes. This coefficient $c_{3}\left(\vec{k}_{1}, \vec{k}_{2}, \vec{k}_{3}\right)$ is given by Klemens [71] using the Grüneisen model developed for thermal expansion:

$c_{3}\left(\bar{k}_{1}, \bar{k}_{2}, \bar{k}_{3}\right)=-i\left(\frac{1}{3 G^{3}}\right)^{2} \cdot \frac{2 M \gamma}{v_{G}} \cdot \omega_{1} \omega_{2} \omega_{3}\left[\sum_{q} e^{i\left(k_{1}+k_{2}-k_{3}\right) \cdot a_{q}}\right]$

(Eq. 17)

where $\gamma$ is the Grüneisen constant; $G$ is the number of lattice sites in the crystal; $M$ is the atomic mass; and $\bar{a}_{q}$ is the lattice constant and $q$ is the lattice site.

When summed over all the lattice sites $\bar{a}_{j}$, the factor in square bracket of (Eq. 17) will 
vanish unless the following conditions are satisfied:

$$
\bar{k}_{1}+\bar{k}_{2}=\bar{k}_{3}
$$

Or

$\bar{k}_{1}+\bar{k}_{2}=\bar{k}_{3}+\bar{b}$

(Eq. 19)

where $\bar{b}$ is one of the reciprocal lattice vectors and has the properties $\bar{a}_{q} \cdot \bar{b}_{j}=2 \pi \delta_{q j}$ and $e^{i \bar{b}_{j} \cdot \bar{a}_{q}}=1$ for any $q$. Under these conditions, (Eq. 17) becomes:

$c_{3}\left(\bar{k}_{1}, \bar{k}_{2}, \bar{k}_{3}\right)=-i\left(\frac{1}{3 G^{3}}\right)^{1 / 2} \frac{2 M \gamma}{v_{G}} \omega_{1} \omega_{2} \omega_{3}$

(Eq. 20)

It has been noted that (Eq. 18) and (Eq. 19) represent two types of three-phonon collision processes which contribute to the perturbation term: Normal process ( $N$-Process) and the Umklapp process (U-Process). These two processes play different roles in the production of thermal resistance and are schematically demonstrated in Figure 28. A $N$-process does not change the direction of energy flow, so it cannot directly contribute to the thermal resistance of a crystal. However, within a specific temperature range, the $N$-process can enhance the ability of defect scattering to maintain thermal equilibrium [69]. On the other hand, the $U$-process can provide a thermal resistance to the energy flow. When the wave vectors of two phonons are larger enough, the resultant wave vector may be outside the first Brillouin zone, the primitive cell in reciprocal space. For phonons, the only meaningful phonons $\bar{k}$ lie in the first Brillouin zone, so that any longer $\bar{k}$ produced in 
collision must be brought back into the first zone by addition of a $\bar{b}$ to maintain the conservation of momentum, which reverses the direction of the resultant wave vector of the produced phonon [68]. Thus, the intrinsic thermal resistance is solely dependent on the $U$-process.

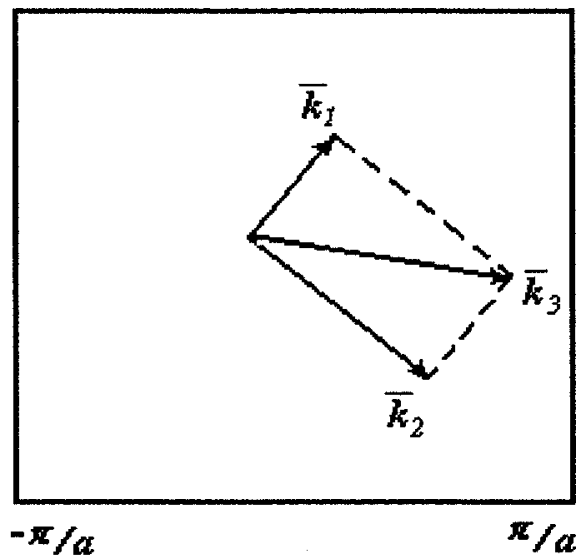

(a) $\bar{k}_{1}+\bar{k}_{2}=\bar{k}_{3}$

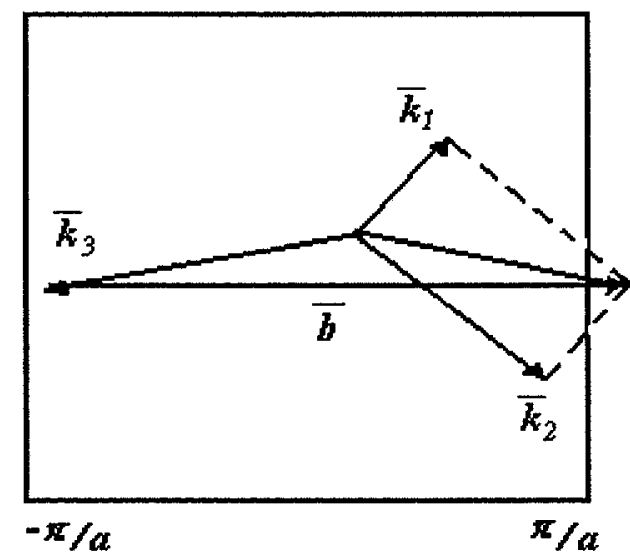

(b) $\bar{k}_{1}+\bar{k}_{2}=\bar{k}_{3}+\bar{b}$

Figure 28 (a) Normal process with $\bar{k}_{1}+\bar{k}_{2}$ within the first Brillouin zone. (b) Umklapp process with $\bar{k}_{1}+\bar{k}_{2}$ lying outside the first Brillouin zone and being brought back by a reciprocal lattice vector.

For thermal resistance caused by the $U$-process, the relaxation time $\tau_{u}$ in the simple cubic lattice has been obtained as [73]:

$$
\frac{1}{\tau_{u}}=\frac{4 \pi \gamma^{2} a}{\sqrt{2}} \frac{k_{B} T}{M v_{G}^{3}} \omega^{2}
$$

The phonon mean free path limited by the U-process can therefore be obtained from the relationship $l_{u}=\tau_{u} \cdot v_{G}$ and is given by: 


$$
l_{u}=\frac{\sqrt{2} M v_{G}^{4}}{4 \pi \gamma^{2} a k_{B} \omega^{2}} \frac{1}{T}
$$

Since the phonon mean free path resulting from phonons interactions is associated with the intrinsic structures of the materials, it is also called the intrinsic phonon mean free path, and thermal conductivity can be also regarded as intrinsic thermal conductivity.

At temperatures higher than the Debye temperature, the specific heat can be expressed as: $c(\omega)=3 k_{B} \omega^{2} / 2 \pi^{2} v_{G}^{3}$

Substituting (Eq. 23) into (Eq. 14), the thermal conductivity is then given [73] by:

$$
\lambda_{i} \cong \frac{3}{4 \pi^{3} \sqrt{2}} \frac{M v_{G}^{2}}{\gamma^{2}} \frac{\omega_{D}}{a} \frac{1}{T}
$$

(Eq. 24) indicates that at high temperatures, for certain materials, such as defect-free zirconia, the intrinsic thermal conductivity is independent of phonon frequency and is inversely proportional to the temperature.

\subsubsection{Phonon scattering by static imperfections}

In order to obtain lower thermal conductivity, one effective way is to reduce the phonon mean free path. One option is to increase phonon scattering. This can be achieved by creating crystal impurities such as vacancies, interstitials, substitution atoms, grain boundaries and defect clusters. 
If a phonon is scattered by a point defect, and transitions from mode $\bar{k}$ into mode $\bar{k}^{\prime}$, it is said that a phonon in mode $\bar{k}^{\prime}$ is created and the one in mode $\bar{k}$ is destroyed. The perturbation Hamiltonian caused by the defect scattering has the form:

$$
H^{\prime}=\sum_{k, k^{\prime}} c_{2}\left(\bar{k}, \bar{k}^{\prime}\right) \xi^{*}\left(\bar{k}^{\prime}\right) \xi(\bar{k})
$$

Similar to $c_{3}\left(\bar{k}_{1}, \bar{k}_{2}, \bar{k}_{3}\right), c_{2}\left(\bar{k}, \bar{k}^{\prime}\right)$ is a critical parameter describing the scattering processes caused by defects. At the scattering site $\bar{x}$, the coefficient $c_{2}\left(\bar{k}, \bar{k}^{\prime}\right)$ is expressed as [72]:

$c_{2}\left(\bar{k}, \bar{k}^{\prime}\right)=\frac{2 M v_{G}}{G} \bar{k}^{\prime} \sum_{x} \delta v_{G} e^{i\left(\bar{k}-\bar{k}^{\prime}\right) \cdot \bar{x}}$

(Eq. 26)

The phonon group velocity $v_{G}$ is associated with $\left(\lambda^{\prime} / M\right)^{1 / 2}$, where $\lambda^{\prime}$ is the force constant of linkage, thus $\delta v_{G}$ can be caused either by a perturbation in force constants as a site, or by atoms of different masses. Considering all the cases of changes in masses, changes in force constants of bonds between that atom and its neighbors, or changes in force constants due to the lattice distribution caused by the impurity atoms or vacancies, Klemens reduced the relationship between $\delta v_{G} / v_{G}$ and other quantities [72]:

$$
\delta v_{G} / v_{G}=\frac{\Delta M}{M}+2 \frac{\Delta F}{F}-2 \gamma \frac{\Delta V}{V}
$$

where $\Delta M / M, \Delta F / F$ and $\Delta V / V$ are the fractional differences in masses, force 
constants and volumes between the impurity and a normal atomic cell in the lattice, respectively. If the impurities are distributed randomly, the defect can be thought of as an isolated impurity $\bar{x}_{0}$. In this situation, the relaxation time $\tau_{P}$ for the scattering resulting from a point defect was obtained and expressed as $[74,75]$ :

$\frac{1}{\tau_{P}}=\frac{a^{3}}{G} \frac{\omega^{4}}{4 \pi v_{G}^{3}}\left(\frac{\Delta M}{M}+2 \frac{\Delta F}{F}-2 \gamma \frac{\Delta V}{V}\right)^{2}$

(Eq. 28)

Likewise, the phonon mean free path caused by point defects can be expressed as:

$\frac{1}{l_{P d}}=\frac{C_{P} a^{3}}{4 \pi v_{G}^{4}} \omega^{4} \Gamma$

(Eq. 29)

where $C_{P}$ is the concentration of point defects; and $\Gamma$ is the scattering factor and defined as:

$\Gamma=[\Delta M / M+2 \Delta F / F-2 \gamma \Delta V / V]^{2}$

(Eq. 30)

It can be seen, from (Eq. 29), that the phonon mean free path caused by defect scattering is independent of temperature, but proportional to the fourth power of the phonon frequency. At high temperatures, the scatterings from the interactions between phonons, point defects and grain boundaries all contribute to the thermal resistance. The combined phonon mean free path can therefore be expressed as:

$\frac{1}{l}=\frac{1}{l_{u}}+\frac{1}{l_{p d}}+\frac{1}{l_{g b}}$ 
where $l_{u}, l_{P_{d}}$ and $l_{g b}$ represent the phonon mean free path cause by intrinsic processes, point defect scattering and grain boundary scattering, respectively. However, the scattering caused by grain boundaries, at high temperatures, has the least effect on the phonon mean free path in conventional materials, but has a significant effect on coatings with grain sizes in the nano-scale.

\subsubsection{Klemens-Callaway model of thermal conductivity}

To calculate thermal conductivity of materials with defects, various models have been developed. Among these models, the most representative are Callaway's and Klemens' thermal conductivity models.

\section{Callaway's model:}

It has been clear that, for the materials containing point defects, the phonon scattering relaxation time is mainly governed by the anharmonic phonon-phonon scattering and the scattering of phonons by point defects. At high temperatures, the phonon-phonon scattering increases with temperature; whereas the phonon-defects scattering is independent of temperature. The combined relaxation time is given by:

$$
\tau(\omega)^{-1}=\tau_{u}^{-1}+\tau_{D}^{-1}=C T \omega^{2}+A \omega^{4}
$$

Where $\tau_{u}$ and $\tau_{D}$ are the relaxation times of phonon-phonon scattering and point defect 
scattering, respectively; $C$ and $A$ are independent of temperature and frequency, but they are dependent on crystal structure and the characters of point defects and can be obtained from (Eq. 21) and (Eq. 28), respectively.

Based on the above thermal conductivity theory, at temperatures higher than the Debye temperature ( $T \geq \theta)$, thermal conductivity in (Eq. 12) becomes:

$$
\lambda=\frac{k_{B}}{2 \pi^{2} v_{G}} \int_{0}^{k_{B} \cdot \theta}{ }^{\hbar} \tau(\omega) \omega^{2} d \omega
$$

Substituting (Eq. 32) into (Eq. 33), and integrating over $\omega$ gives [76]:

$$
\lambda=\frac{k_{B}}{2 \pi^{2} v_{G}(A C T)^{2}} \tan ^{-1}\left[\frac{k_{B} \theta}{\hbar}\left(\frac{A}{C T}\right)^{2}\right]
$$

In the limit of perfect crystal structure, $A \rightarrow 0$. The intrinsic thermal conductivity $\lambda_{u}$ can be reduced from (Eq. 34) to give:

$$
\lambda_{u}=\frac{k_{B}^{2} \theta}{2 \pi^{2} v_{G} \hbar C T}
$$

To evaluate thermal conductivities of ceramic materials containing point defects, Callaway $[76,77]$ introduced thermal resistance $W$ as the inverse of $\lambda$ so that the calculation can be simplified due to the additivity of thermal resistances resulting from different scattering mechanisms. Assuming $W_{u}$ is the reverse of $\lambda_{u}$ resulting from phonon- phonon scattering in a pure crystal, a ratio of resistance from phonon-phonon 
scattering to total resistance of real material is then given by:

$$
\frac{W_{u}}{W}=\frac{\hbar}{k_{B} \theta}\left(\frac{C T}{A}\right)^{\frac{1}{2}} \tan ^{-1}\left[\frac{k_{B} \theta}{\hbar}\left(\frac{A}{C T}\right)^{\frac{1}{2}}\right]
$$

(Eq. 36)

In the case of small defect scattering, the (Eq. 36) can be expanded to give:

$W=W_{u}\left(1+\frac{k_{B}^{2} \theta^{2}}{3 \hbar^{2}} \frac{A}{C T}\right)=W_{u}+\frac{2 \pi^{2} v_{G} \theta A}{3 \hbar}$

(Eq. 37)

The second term in (Eq. 37) is independent of temperature and can be thought to be the thermal resistance $W_{D}$ caused by point defect scattering. Therefore,

$$
W_{D}=\frac{2 \pi^{2} v_{G} \theta A}{3 \hbar}
$$

(Eq. 38)

\section{Klemens' model:}

Klemens proposed a phonon frequency $\omega_{0}$ at which $l_{u}\left(\omega_{0}, T\right)=l_{p d}\left(\omega_{0}\right)$, the total phonon mean free path can then be written as [75]:

$l_{p}(\omega)=l_{i}(\omega, T)\left[1+\left(\omega / \omega_{0}\right)^{2}\right]^{-1}$

(Eq. 39)

Substituting (Eq. 39) into (Eq. 14), the reduction in thermal conductivity due to the imperfection can be expressed as:

$\lambda=\lambda_{i}-\delta \lambda_{p d}$ 
And

$\delta \lambda_{p d}=\lambda_{i}\left[1-\left(\omega_{0} / \sqrt{2} \omega_{D}\right) \arctan \left(\sqrt{2} \omega_{D} / \omega_{0}\right)\right]$

(Eq. 41)

From the thermal conductivity theory summarized above, it becomes apparent that the following approaches can be taken to create thermal barrier coating systems with reduced thermal conductivity:

- Introduction of crystal defects through doping using atoms with different mass, ionic radius or valence from the host atom to increase scattering centers;

- Modification of coating microstructure such as reducing the grain sizes or creating porous structures;

- Selection of new materials with low intrinsic thermal conductivity.

\subsubsection{Radiation Transport through Zirconia Based Ceramic Coatings}

It is well known that, in ceramic based materials, there are two heat transfer mechanisms: one is thermal conduction which has been discussed in section 2.6 .1 ; another is thermal radiation transport, which is dependent on the spectral properties of the ceramic materials and the radiation from the hot gases due to high operating temperatures in turbine engines.

\subsubsection{Radiation properties of zirconia based ceramics}

The spectral hemispherical transmittance and emittance of single crystal $13.5 \mathrm{YSZ}$ specimens of various thicknesses at room temperature were measured and given in Figure 
29 [78]. The spectral properties of YSZ can be divided into three regions. As seen, in region I $(0.3 \sim 5 \mu \mathrm{m})$, the YSZ exhibits a high transmittance with low absorption; and in region II $(5 \sim 10 \mu \mathrm{m})$, partial absorption and partial transmission were observed. In region III, the YSZ shows an opposite trend as compared to what is seen in region I. Therefore, thermal radiation within the wavelength range of $0.3 \sim 10 \mu \mathrm{m}$ can either transmit directly through zirconia based $\mathrm{TBC}$ coatings to the metal substrate, or be absorbed and re-emitted, depending on the wavelength of the radiation. For radiation beyond $10 \mu \mathrm{m}$, the coating is opaque and all the radiation within this wavelength range is absorbed and transformed into heat and results in temperature increase to the ceramic materials.

The radiative emission is strongly dependent on temperature. When the operating temperatures are not high enough, the wavelength of the radiation emitted from the hot gases will be beyond the transparent range of the ceramics and will be absorbed by the ceramics. In this situation, thermal conduction dominates in the heat transfer through the coatings. With increase in gas temperatures the radiation emitted from hot gas shifts to the shorter wavelength range and most radiation may fall within the transparent region of zirconia based ceramics. Therefore, a significant heat flux through the coatings to the metal substrate will occur due to thermal radiation and will result in a further increase in temperature on metal substrates. Figure 30 shows that at typical operating temperatures between $1700-2000 \mathrm{~K}$, almost $80 \%$ of the radiation flux emitted from the hot gases will fall within $0.3 \sim 10 \mu \mathrm{m}$ wavelength range, which is the transparent or semi-transparent regions for YSZ. In this case, temperature increase on the metallic substrate caused by 
thermal radiation can become significant and cannot be ignored.
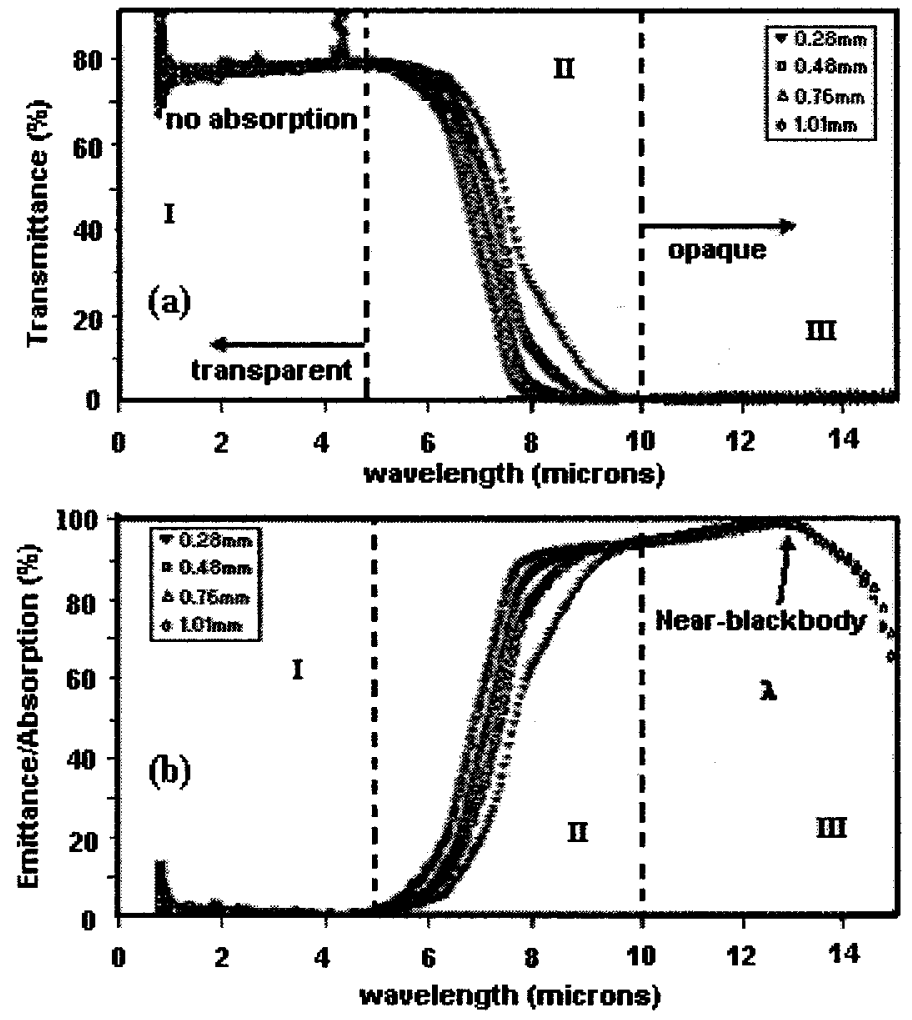

Figure 29 Room-temperature (a) hemispherical transmittance and (c) emittance / absorption along (100) direction of single crystal 13.5 YSZ specimens with various thicknesses [78].

This effect has been observed by several researchers $[38,79,80]$. The measured thermal conductivities are found to contradict this trend and the increases in thermal conductivity with temperature are observed in both lanthanum zirconates and zirconia-based ceramic coatings. According to thermal conductivity theory, the intrinsic thermal conductivity of ceramic coatings has an inverse dependence on temperature. However, values of measured thermal conductivities are found to contradict this trend. Increase in thermal 67 
conductivity with rising temperature has been observed and is attributed to thermal radiation since the $\mathrm{TBC}$ materials are partially or fully transparent to the thermal radiation at typical engine operating temperatures.

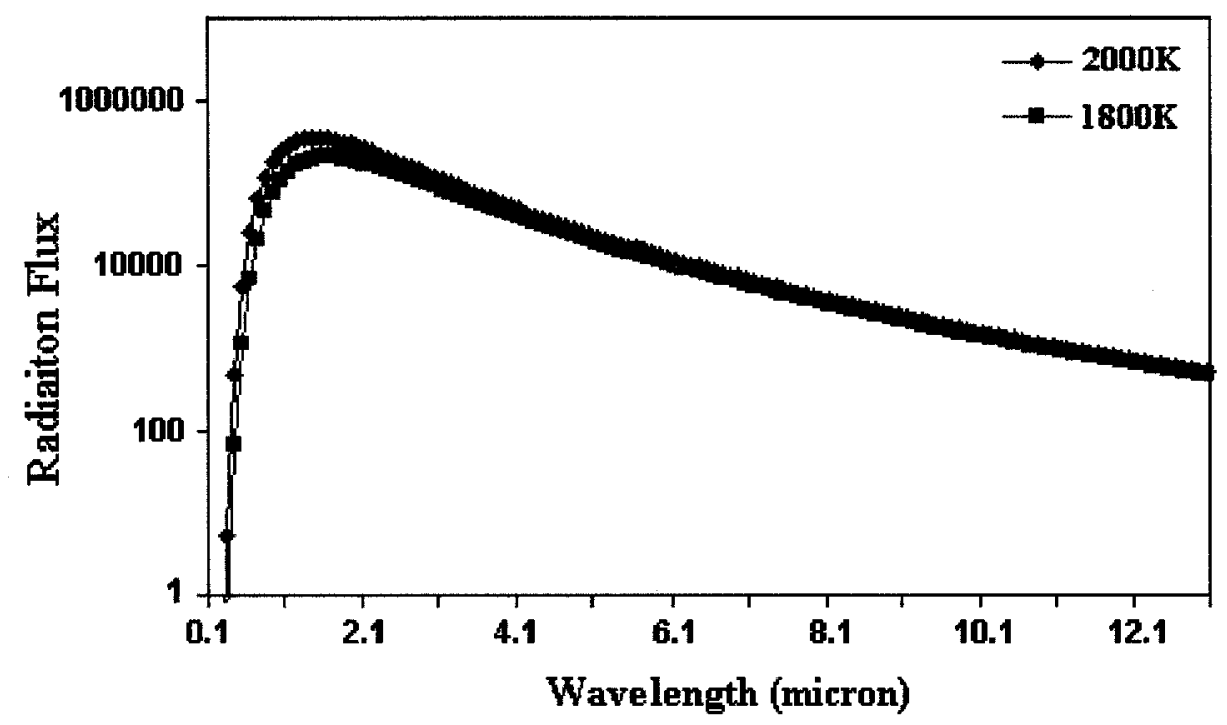

Figure 1 Emission energy with a black body spectrum at different temperatures.

The temperature increase on the metallic substrate caused by thermal radiation has been evaluated by Siegel and Spuckler in their analysis of thermal radiation effects in turbine engine TBCs [81]. In this study, the engine thermal environment was summarized with regard to factors affecting radiative heat transfer: Radiation transport through thermal barrier coatings to the metal component originates from two sources: (1) external radiation from hot gases or soot, which is directly incident onto the coating surface and is transmitted to the substrate; and (2) internal radiation emission within the hot coating itself when heated by convection from combustion gases, conduction within the coating system or absorption of external and internal radiation. The energy equations including 
radiation transfer within the coatings were established [81]. By solving these equations, it was found that if the hot gas temperature is $2000 \mathrm{~K}$, the temperature increase on the metallic substrate resulting from radiation effects could reach as high as $50^{\circ} \mathrm{C}$ as compared to that of an opaque coating, as shown in Figure 31. Thus, at high temperatures, thermal radiation is a very important heat transport mechanism.

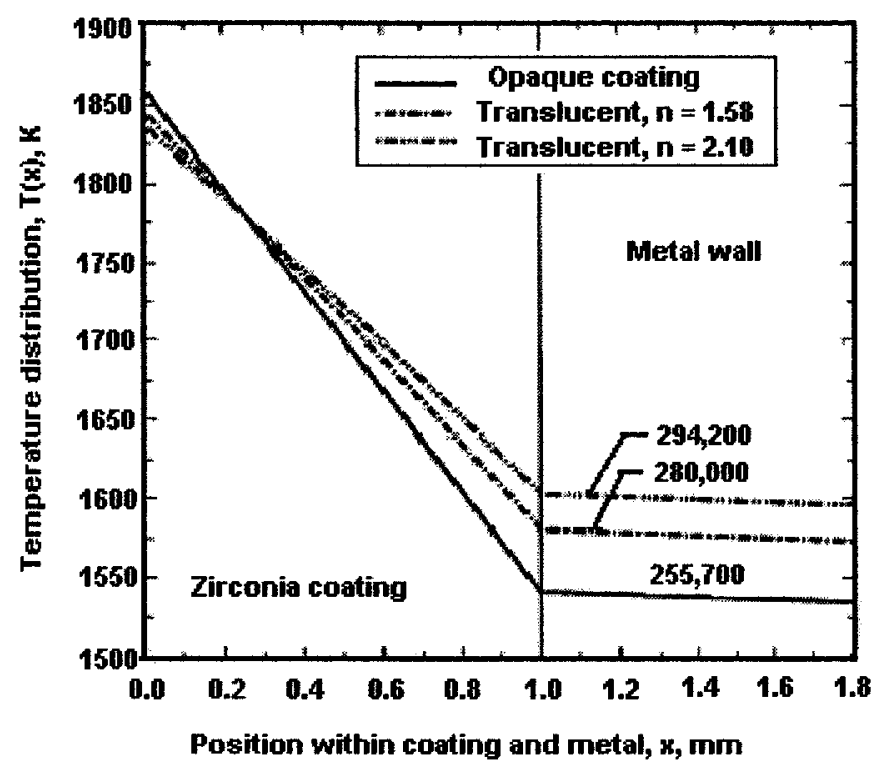

Figure 31 Temperature distributions calculated in a zirconia thermal barrier coating on the wall of a combustor compared with an opaque thermal barrier coating [81].

\subsubsection{Radiation transfer within ceramic coating materials}

Unlike that in a single crystal YSZ, thermal radiation transferred through YSZ thermal barrier coatings deposited by either plasma spray or EB-PVD processes will experience scattering caused by pores and other defects in the coatings. Radiation transmitted through such ceramic coatings experiences absorption, emission, and scattering. According to radiation transfer theory [82], at the direction $\omega$ of solid angle, the radiation 69 
intensity $i_{\lambda}\left(\kappa_{\lambda}, \omega\right)$ along a path $x$ within a medium can be expressed as:

$\frac{d i_{\lambda}\left(\kappa_{\lambda}, \omega\right)}{d \kappa_{\lambda}}+i_{\lambda}\left(\kappa_{\lambda}, \omega\right)=I_{\lambda}\left[\kappa_{\lambda}, \omega\right]$

(Eq. 42)

$I_{\lambda}\left(\kappa_{\lambda}, \omega\right)$ in (Eq. 42) is the source function, representing the sum of the gain by local blackbody emission $i_{\lambda b}\left(\kappa_{\lambda}\right)$ and the gain by scattering in $x$ the direction. $I_{\lambda}\left(\kappa_{\lambda}, \omega\right)$ is given by:

$$
I_{\lambda}\left(\kappa_{\lambda}, \omega\right)=\left(1-\Omega_{\lambda}\left(\kappa_{\lambda}\right)\right) \cdot i_{\lambda b}\left(\kappa_{\lambda}\right)+\frac{\Omega_{\lambda}\left(\kappa_{\lambda}\right)}{4 \pi} \int_{b_{i}=0}^{4 \pi} i_{\lambda}\left(\kappa_{\lambda}, \omega_{i}\right) \Phi_{\lambda}\left(\omega, \omega_{i}\right) d \omega_{i}
$$

where $\Phi_{\lambda}\left(\omega, \omega_{i}\right)$ is the phase function; and $\omega_{i}$ is the incident solid angle, representing the radiation from the direction $\omega_{i}$ and scattered into $\omega$. And

$d \omega=\sin \theta d \theta d \varphi$

(Eq. 44)

where $\theta$ and $\varphi$ are polar and circumferential angles of the radiation within the medium. The subscript $\lambda$ means that radiation intensity is a function of wavelength. $\kappa_{\lambda}$, a function of $x$, is the optical depth defined by:

$$
\kappa_{\lambda}(x)=\int_{0}^{x}\left[\alpha_{\lambda}\left(x^{*}\right)+\sigma_{s \lambda}\left(x^{*}\right)\right] d x^{*}=\int_{0}^{x} \mathrm{~K}_{\lambda}\left(x^{*}\right) d x^{*}
$$

where $\mathrm{K}_{\lambda}\left(x^{*}\right)=\alpha_{\lambda}\left(x^{*}\right)+\sigma_{s \lambda}\left(x^{*}\right)$, is the extinction coefficient; $\alpha_{\lambda}\left(x^{*}\right)$ and $\sigma_{s \lambda}\left(x^{*}\right)$ are the absorption coefficient and scattering coefficient respectively; and $\Omega_{\lambda}\left(\kappa_{\lambda}\right)$ in (Eq. 43) is termed the albedo and is defined as: 


$$
\Omega_{\lambda}(x)=\frac{\sigma_{s \lambda}(x)}{\alpha_{\lambda}(x)+\sigma_{s \lambda}(x)}
$$

The intensity emitted from the blackbody within the coating is given by:

$$
i_{\lambda b}=\frac{2 n_{s}^{2} C_{1}}{\lambda^{5}\left(e^{C_{2} / \lambda T(x)}-1\right)}
$$

where, $T(x)$ is the temperature at $x$ position in the coating, $n_{s}$ is the refractive index of the coating, and $C_{I}=0.59552137 \times 10^{8} \mathrm{~W} \cdot \mu \mathrm{m}^{4} /\left(\mathrm{m}^{2} \cdot s r\right), C_{2}=14387.752 \mu \mathrm{m} \cdot \mathrm{K}$.

The coating layer can be simplified as a plane due to its large dimensions relative to its thickness. As such, the heat transfer from the coating surface to the metal substrate can be simplified as a one-dimensional model. Therefore, (Eq. 44) becomes:

$d \omega=2 \pi \sin \theta d \theta=2 \pi d \mu$

where $\mu=\cos \theta$. Let the intensity of radiation transporting in a direction towards the metal substrate within the ceramic coating at position $x$ be denoted as $i_{\lambda}^{+}\left(\kappa_{\lambda}, \mu\right)$, and the intensity in the opposite direction at position $x$ be denoted as $i_{\lambda}^{-}\left(\kappa_{\lambda},-\mu\right)$, as shown in Figure 32. The variations of radiation intensity through the ceramic coating can then be expressed as:

$i_{\lambda}^{+}\left(\kappa_{\lambda}, \mu\right)=i_{\lambda}^{+}(0, \mu) e^{-\kappa_{\dot{\lambda}} / \mu}+\frac{1}{\mu} \int_{0}^{\kappa_{i}} I_{\lambda}\left(\kappa_{\lambda}^{*}\right) e^{-\left(\kappa_{\lambda}-\kappa_{\lambda}^{*}\right) / \mu} d \kappa_{\lambda}^{*}$ 
where $0 \leq \mu \leq 1 ; i_{\lambda}^{+}(0, \mu)$ is the radiation intensity at the coating surface in the positive direction; and $d \kappa_{\lambda}^{*}=\mathrm{K}_{\lambda}\left(x^{*}\right) d x^{*}$.

$i_{\lambda}^{-}\left(\kappa_{\lambda}, \mu\right)=i_{\lambda}^{-}\left(\kappa_{D \lambda}, \mu\right) e^{-\left(\kappa_{D \lambda}-\kappa_{\lambda}\right) /(-\mu)}-\frac{1}{\mu} \int_{\kappa_{\lambda}}^{\kappa_{D \lambda}} I_{\lambda}\left(\kappa_{\lambda}^{*}\right) e^{-\left(\kappa_{\lambda}^{*}-\kappa_{\dot{\lambda}}\right) /(-\mu)} d \kappa_{\lambda}^{*}$

where $-1 \leq \mu \leq 0 ; \kappa_{D \lambda}$ is the optical depth at the position $x=D$, where $D$ is the thickness of the thermal barrier coating; $i_{\lambda}^{-}\left(\kappa_{D \lambda}, \mu\right)$ is the radiation intensity in the negative direction at the interface between the coating and metal substrate in the negative direction.

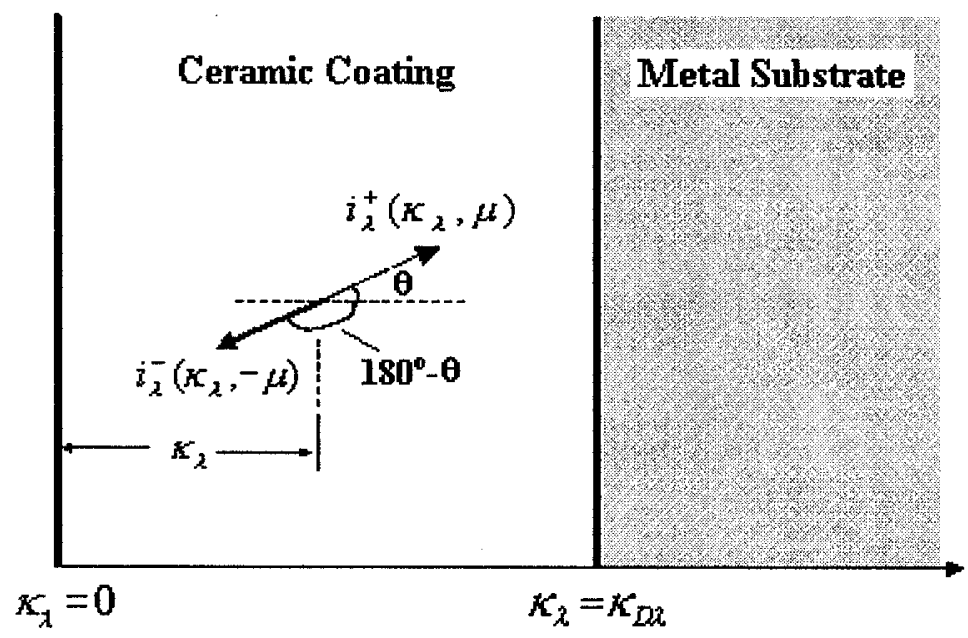

Figure 32 Schematic diagram of radiation intensity variation transporting through a ceramic coating.

The spectral radiation flux through the coating can be evaluated by integrating the intensity of radiation over all directions:

$q_{r \lambda}\left(\kappa_{\lambda}\right) d \lambda=2 \pi d \lambda \int_{\mu=0}^{\lambda}\left[i_{\lambda}^{+}\left(\kappa_{\lambda}, \mu\right)-i_{\lambda}^{-}\left(\kappa_{\lambda},-\mu\right)\right] \mu d \mu$ 
As seen from equation (Eq. 51), the value for the $\left[i_{\lambda}^{+}\left(\kappa_{\lambda}, \mu\right)-i_{\lambda}^{-}\left(\kappa_{\lambda},-\mu\right)\right]$ term should be kept as low as possible in order to reduce the total radiation flux into the TBC system. This can be achieved by either increasing the reflected radiation, i.e. maximizing $i_{\lambda}^{-}\left(\kappa_{\lambda},-\mu\right)$, or by increasing both the absorption coefficient $\alpha_{\lambda}(x)$ and the scattering coefficient $\sigma_{s \lambda}(x)$ to attenuate $i_{\lambda}^{+}\left(\kappa_{\lambda}, \mu\right)$ [82]. The most effective method to increase the reflectivity of the radiation is to use multiple layered coatings with alternating layers of materials with high and low refractive indices. The design methodology of such coating structures will be detailed in this study.

\subsection{Methods to Reduce Thermal Conduction through TBCS}

It is well known, from thermal conductivity theory, that to reduce thermal conduction of TBC materials, one effective method is to increase the phonon scattering centers by doping or modifying the coating's microstructure. Another is to find new materials with low intrinsic thermal conductivity. In the last decade, a host of new materials, novel microstructures, and advanced coating application technologies has led to improved thermal insulation capability, durability and stability at higher temperature, as well as better mechanical properties, which will be described in detail in the flowing section.

\subsubsection{Introduction of Crystal Defects}

Zirconium oxide has a lower intrinsic thermal conductivity than other ceramics such as $\mathrm{Al}_{2} \mathrm{O}_{3}$. Stabilized zirconia has an even lower thermal conductivity than pure zirconia due 
to the introduction of crystal defects. The addition of the metal oxides with cation valence less than $4+$ to zirconia will create more $\mathrm{O}^{2-}$ vacancies in the ionic lattice in order to maintain electrical neutrality. These vacancies strongly scatter phonons by virtue of both missing mass and missing interatomic linkage and result, therefore, in a decrease in the thermal conductivity. Alperine [38] and Nicholls [5] have reported that the measured thermal conductivities of bulk zirconia ceramic and zirconia $\mathrm{TBC}$ are a function of the yttria content. Figure 33 shows that the thermal conductivity decreases as the yttria content increases. However, further reduction in thermal conductivity of YSZ through yttria addition is limited by the need to stabilize the zirconia diffusionless tetragonal $t^{\prime}$ phase structure, since the high dopant concentration reduces the toughness of coatings [83]. Furthermore, the addition of tetravalent oxides, such as ceria $\left(\mathrm{CeO}_{2}\right)$, to the zirconia has also shown a reduction in thermal conductivities without creating any oxygen vacancies. It has been reported that the $17 \mathrm{~mol} \%$ ceria stabilized zirconia has the same thermal conductivity as that of $4 \mathrm{~mol} \%$ yttria stabilized zirconia [84]. This suggests that replacement of zirconium atoms by other dopant cations can also create scattering centers and result in the reduction of thermal conductivity, although these effects are much weaker than that of vacancies.

For the $\mathrm{ZrO}_{2}-\mathrm{M}_{2} \mathrm{O}_{3}$ binary system, where $\mathrm{M}$ represents a trivalent metal ion, a semiempirical phonon model indicated that there is a linear trend of decreasing thermal conductivity with increasing cation size of the dopant for the partially stabilized YPSZ, DyPSZ and SmPSZ. This model was confirmed experimentally $[1,30]$. However, the study of phase stability of $\mathrm{ZrO}_{2}-\mathrm{M}_{2} \mathrm{O}_{3}$ binary system at the same dopant concentration 
showed that zirconia doped with larger or smaller cations than $\mathrm{Yb}$ and $\mathrm{Y}$ cations is not stable, as shown in Figure 11. The cation sizes of $\mathrm{Yb}$ and $\mathrm{Y}$ are interestingly close to the size of the coordinating oxygen cube and therefore increase phase stability of doped zirconia.

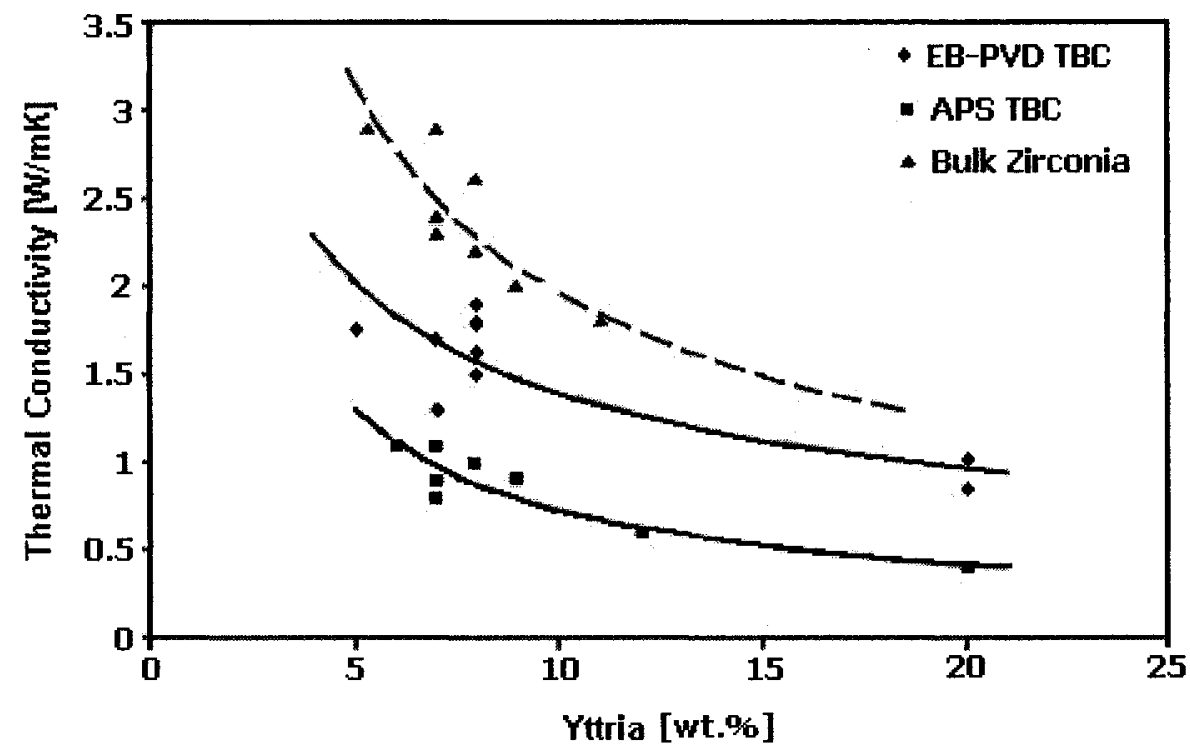

Figure 33 Thermal conductivities of zirconia ceramic and zirconia thermal barrier coatings as a function of the yttria content [5]

To incorporate more scattering centers without affecting the crystal structure and phase stability, transition metal oxides and rare earth oxides have been used either singly or together as dopants for YSZ. For example, Tamarin et al. [85] added five types of metal oxides with total amounts less than $5 \%$ to $\mathrm{ZrO}_{2}-8 \mathrm{Y}_{2} \mathrm{O}_{3}$, and reductions of $30-40 \%$ in thermal conductivity were achieved for EB-PVD coatings compared to $\mathrm{ZrO}_{2}-8 \mathrm{Y}_{2} \mathrm{O}_{3}$ when measured at elevated temperatures.

According to thermal conductivity theory, additions of elements with high atomic mass 
will provide more effective scattering centers. Based on this, Rickerby and his coworkers [84] examined five dopants: $\mathrm{Er}_{2} \mathrm{O}_{3}, \mathrm{NiO}, \mathrm{Nd}_{2} \mathrm{O}_{3}, \mathrm{Gd}_{2} \mathrm{O}_{3}$ and $\mathrm{Yb}_{2} \mathrm{O}_{3}$. For a $4 \mathrm{~mol} \%$ addition of each oxide, thermal conductivities of TBCs doped with Neodymia and Gadolinia dropped to $0.89 \mathrm{~W} / \mathrm{mK}$ and $0.86 \mathrm{~W} / \mathrm{mK}$, respectively, when measured at room temperature. For an $8 \mathrm{~mol} \%$ addition of $\mathrm{Er}_{2} \mathrm{O}_{3}$ and $\mathrm{Nd}_{2} \mathrm{O}_{3}, 32 \%$ and $52 \%$ reductions in thermal conductivity were achieved respectively. However, there is no clear trend indicating how the ionic size or mass of the dopant cations affects thermal conductivities. These experimental results might be skewed by the pores existing in the EB-PVD coatings. Thus, fully densed co-doped materials may give more accurate results with regard to the intrinsic properties of the doped oxides.

To study the effects of mass and ionic radius of doped cations on the thermal conductivity of zirconia without the influence of pores in the material, densified zirconia co-doped with tantala and yttria or niobia and yttria, was investigated by Raghavan et al. [86]. In this study, $\mathrm{Ta}_{2} \mathrm{O}_{5}$ or $\mathrm{Nb}_{2} \mathrm{O}_{5}$ was added in the same amount as $\mathrm{Y}_{2} \mathrm{O}_{3}$ to annihilate oxygen vacancies. Both doped cations were expected to scatter phonons due to the difference in ionic radius, mass difference and atomic bonding. The tantalum ion was expected to be a more effective dopant than the niobium ion due to its twofold mass difference. The experimental results showed both measured thermal conductivities of the $8.52 \mathrm{~mol} \%$ tantala $/ 9.93 \mathrm{~mol} \%$ yttria co-doped zirconia and $9.64 \mathrm{~mol} \%$ niobia $/ 9.58 \mathrm{~mol} \%$ yttria co-doped zirconia are very close to that of $4 \mathrm{~mol} \% \mathrm{YSZ}$. This indicated that the differences in ionic mass and radius between the doped ions and zirconium ions, as shown by the data in Table 4, affect the thermal conductivity, although the effect may not 
be as strong as that of vacancies. However, there was little difference in thermal conductivity between the tantala / yttria co-doped zirconia and niobia / yttria co-doped zirconia, indicating that the effect of mass difference is much smaller than that of ionic difference.

To reduce the thermal conductivity and at the same time to improve the coatings sintering resistance, a multi-component defect-clustering approach was employed [87, 88]. In this approach, a group of selected oxides including $\mathrm{Nd}_{2} \mathrm{O}_{3}\left(\mathrm{Gd}_{2} \mathrm{O}_{3}, \mathrm{Sm}_{2} \mathrm{O}_{3}\right)$ and $\mathrm{Yb}_{2} \mathrm{O}_{3}$ $\left(\mathrm{Sc}_{2} \mathrm{O}_{3}\right)$ were doped into conventional zirconia and hafnia yttria to create thermodynamically stable, highly defective lattice structures with essentially immobile defect clusters and/or nanoscale ordered phases. With this nanoscaled defect clustering structure, the cluster containing coating systems exhibited much lower thermal conductivity than conventional YSZ coatings or singly doped YSZ coatings at both room temperature and high temperatures; and thermal conductivity of the cluster oxide coatings generally decreased with increasing total dopant concentration, as shown in Figure 34.

Based on the review of doped or co-doped zirocnia, it is observed that increases in crystal defects, such as more oxygen vacancies, differences in mass or ionic radius, can effectively reduce thermal conductivity of the zirconia and other ceramic materials. However, the practical use of these doped coating materials may be limited by their thermal cyclic life and durability. For example, thermal cycling tests on multicomponent rare earth oxide doped TBCs showed that the cyclic life of doped coatings generally decreased with increasing dopant concentration [89]. Among all the doped zirconia based ceramic materials, 4mol\% YSZ exhibited excellent furnace cyclic 
durability. The $t^{\prime}$ phase containing yttria has a better cyclic resistance and higher fracture toughness than the cubic phase with higher yttria content.

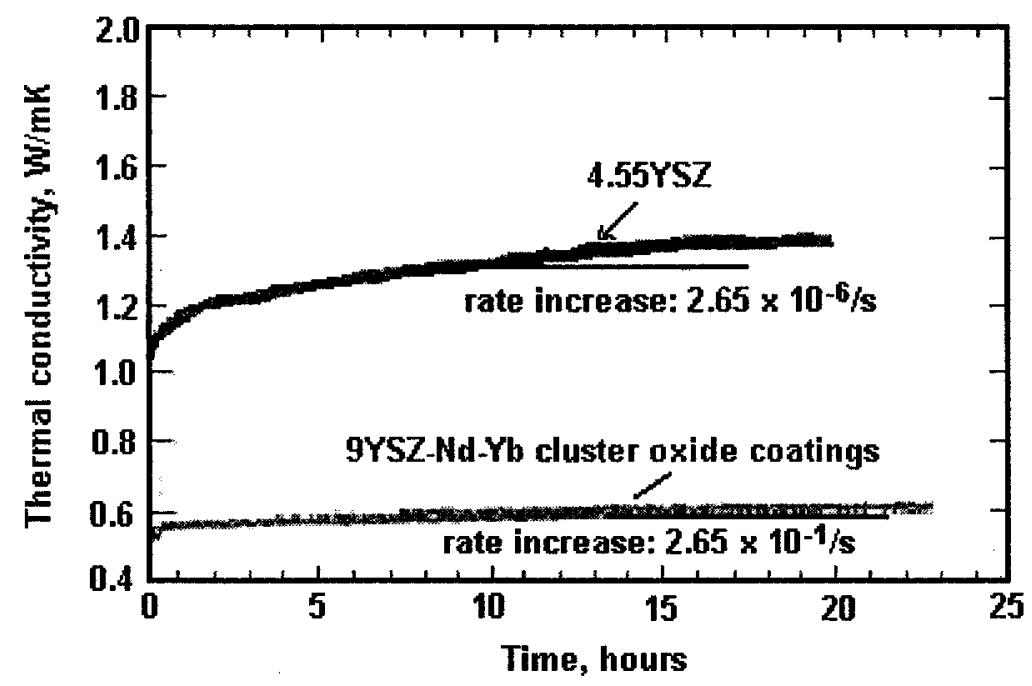

Figure 34 Thermal conductivity of plasma-sprayed oxide cluster 9YSZ-Nd-Yb coatings and a baseline $\mathrm{ZrO}_{2}-4.55$ mol\% $\mathrm{Y}_{2} \mathrm{O}_{3}$ (4.55YSZ) coating as a function of test time measured at a surface temperature of $1316^{\circ} \mathrm{C}$ [89].

\subsubsection{Modification of Thermal Barrier Coating Microstructures}

The microstructure and pore morphologies of thermal barrier coatings have a significant effect on thermal conductivity. As discussed previously, plasma sprayed coatings provide greater resistance to heat flow than EB-PVD coatings due to their different pores morphology. To couple the pore morphology that offers the highest impedance to heat flow with the columnar microstructure to achieve improved thermal and mechanical performance, a novel thermal barrier coating that exhibited a columnar structure with zigzag morphology pores is presented in Figure 35 [90]. The coating was deposited by 
electron beam-directed vapor deposition (EB-DVD) technology. Several EB-DVD coatings, having a different zig-zag column morphology were examined and the thermal conductivity of the EB-DVD deposited zig-zag TBC with a $13.1 \mu \mathrm{m}$ wavelength had the minimum value of $0.8 \mathrm{~W} / \mathrm{mK}$ at room temperature [91].

Layer interfaces were introduced into each column of an EB-PVD coating [5, 45]. As seen in Figure 36, the layers in each column are approximately parallel to the coating surface, and the densities are changed from layer to layer by switching the D.C bias applied to the substrate between high and low levels during deposition. The periodicities of the layers are chosen to lie between $0.2 \sim 2.0 \mu \mathrm{m}$ and centered at a wavelength of $0.7 \mu \mathrm{m}$. The combination of the layer boundaries and variations in density is thought to be able to reduce both phonon and photon transport. The measured thermal conductivity of this microstructure indicated that a $37-45 \%$ reduction in thermal conductivity was achieved, depending on the process variables used, as shown in Figure 37.

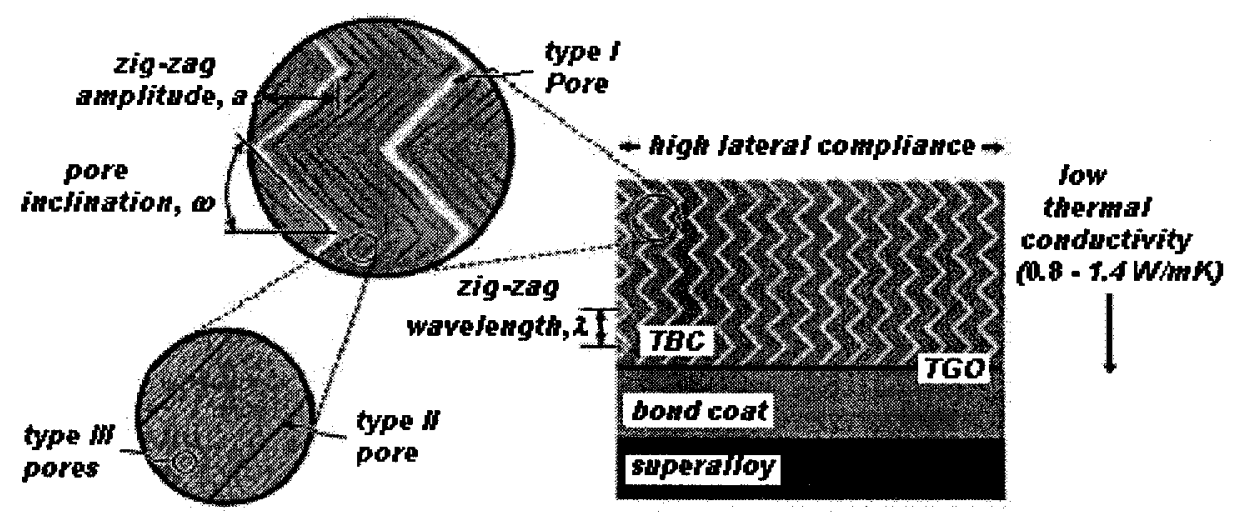

Figure 35 Zig-zag pores at different scale levels [90]. 


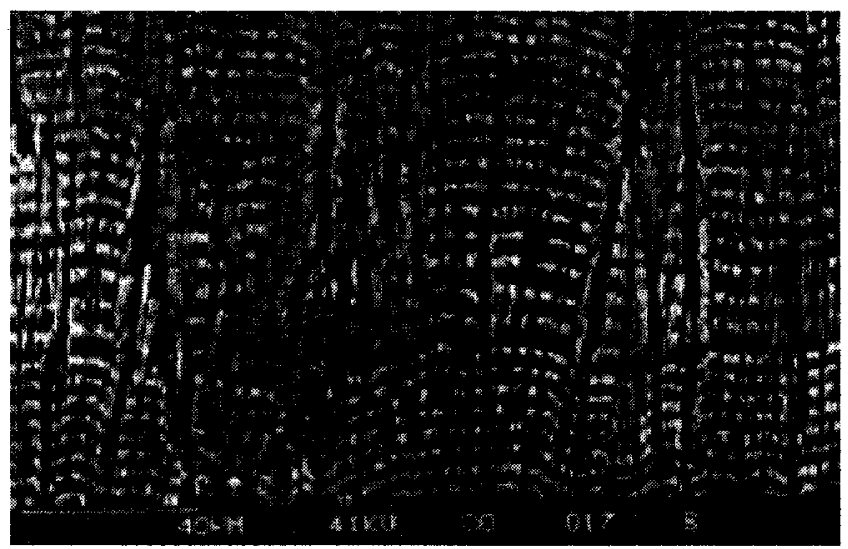

Figure 36 Layered structured EB-PVD TBC introduced by Plasma Assisted Physical Vapor Deposited (PAPVD) processing of the ceramic [5].

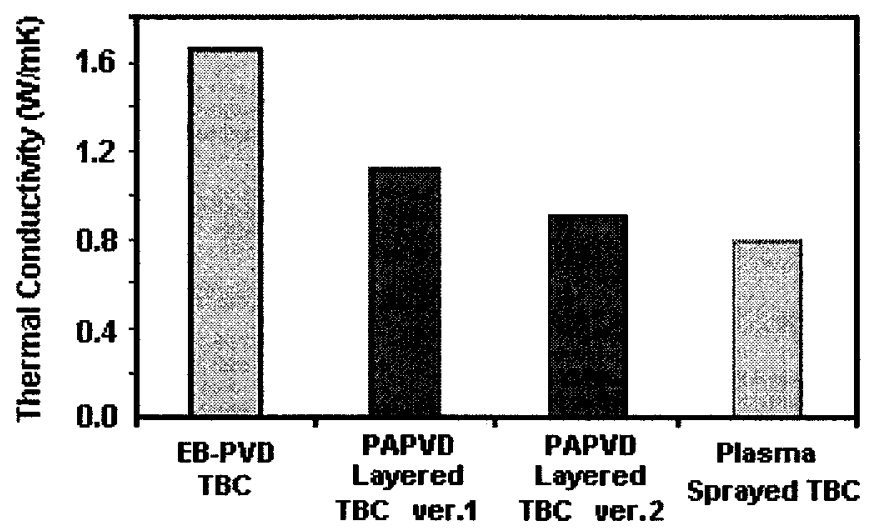

Figure 37 Thermal conductivities of layered EB-PVD TBC systems compared to the EB-PVD and thermal sprayed TBCs [5].

As described earlier, the inner layer of EB-PVD TBC exhibits lower thermal conductivity than the columnar grained outer layer. Based on these findings, a modified coating structure was developed by creating periodic strain fields within the TBC using a "shutter" method during the EB-PVD deposition process. It consists of multiple layers, each layer having a microstructure similar to that of the inner zone of initial EB-PVD 
coating, as shown in Figure 38.

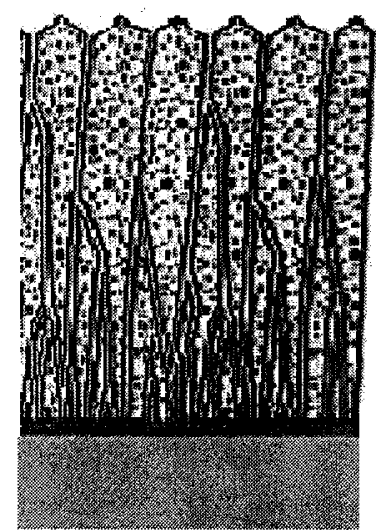

(a) Single Layered

Columnar Coating

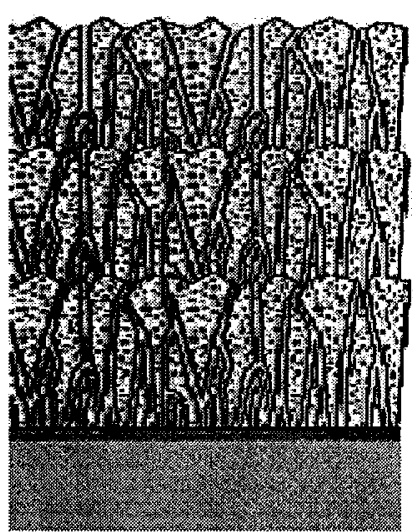

(b) Multilayered Columnar Coating

Figure 38 (a) Typical standard vapor phase columnar structure; and (b) modified columnar microstructure with multiple interfaces [45].

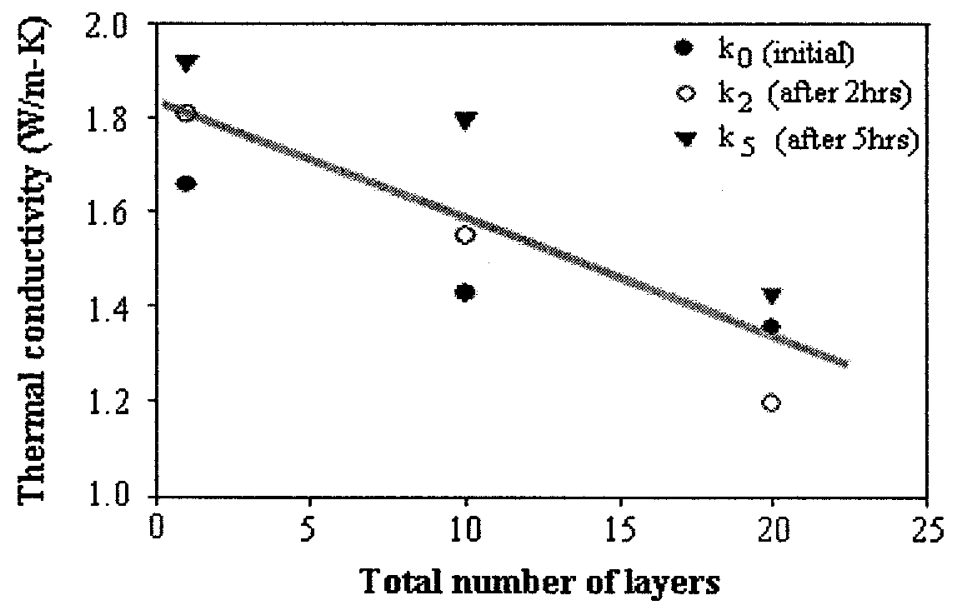

Figure 39 Thermal conductivity of EB-PVD coatings as a function of total number of layers produced by the "shutter" method, measured at various stages of testing, where $\mathrm{k}_{0}$ $=$ as deposited, $\mathrm{k}_{2}=$ after $2 \mathrm{hrs}$, and $\mathrm{k}_{5}=$ after 5hrs of testing [45].

The increased phonon scattering centers caused by a number of interfaces, grain 81 
boundaries as well as microporosity within each layer therefore resulted in a reduction in thermal conductivity. Figure 39 shows the measured thermal conductivity of EB-PVD coatings as a function of the total number of layers produced by the "shutter" method. There is an observed $30 \%$ reduction in thermal conductivity for coatings with 20 layers [45].

\subsubsection{Development of New Generation of TBC Materials}

It is well known that increasing operating temperatures will improve the performance of gas turbine and diesel engines. However, at temperatures above $1200^{\circ} \mathrm{C}$, conventional YSZ is unable to provide thermal protection to metallic substrates as it exhibits destabilization of the tetragonal t' phase to tetragonal and cubic on extended exposure. On cooling, the tetragonal phase will transform to monoclinic phase, which results in cracking $[64,83,92]$. In addition, at high temperatures, YSZ exhibits a sintering effect, which will cause an increase in Young's modulus and thermal conductivity [60]. Thus, the development of new thermal barrier coating materials, which can sustain temperatures beyond $1200^{\circ} \mathrm{C}$, is required.

The rare earth zirconates, such as $\mathrm{La}_{2} \mathrm{Zr}_{2} \mathrm{O}_{7}, \mathrm{Nd}_{2} \mathrm{Zr}_{2} \mathrm{O}_{7}$, and $\mathrm{Ga}_{2} \mathrm{Zr}_{2} \mathrm{O}_{7}$, have been found to be the most promising TBC materials for the future. These zirconates have a typical composition of $\mathrm{A}_{2} \mathrm{~B}_{2} \mathrm{O}_{7}$ and have cubic pyrochlore structures, as shown in Figure 40 [93, 94]. For comparison, the fluorite structure of $\mathrm{ZrO}_{2}$ is also shown in the figure. They assume a single pyrochlore phase which is stable up to the melting point at $2300^{\circ} \mathrm{C}$ [95]. Additionally, rare earth zirconates are not oxygen transparent and have very low thermal 
conductivity values.

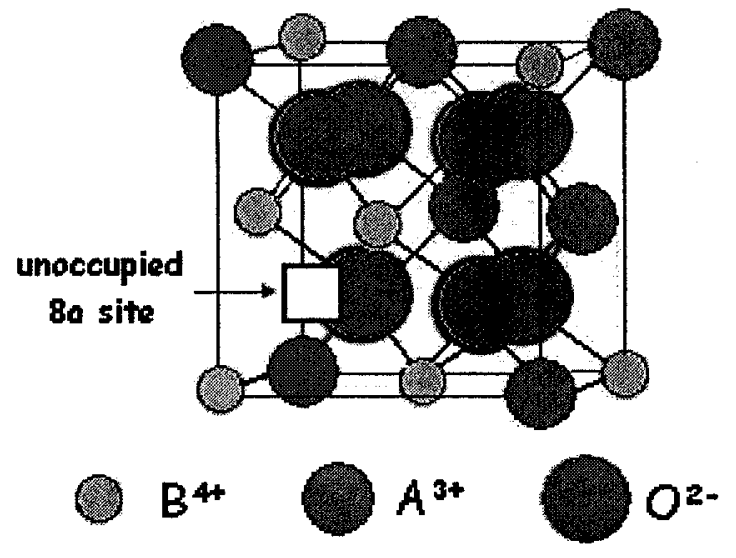

(a) Pyrochlore structure of A.2B207

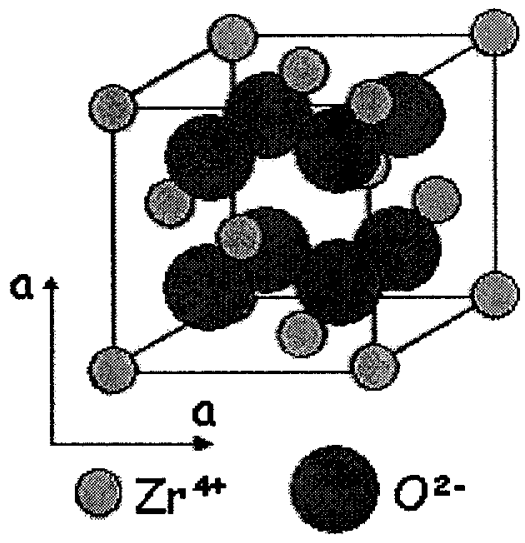

(b) Fluorite structure of Zroz

Figure 40 Schematics of (a) the partial unit cell of the pyrochlore structure [93], (b) unit cell of fluorite structure.

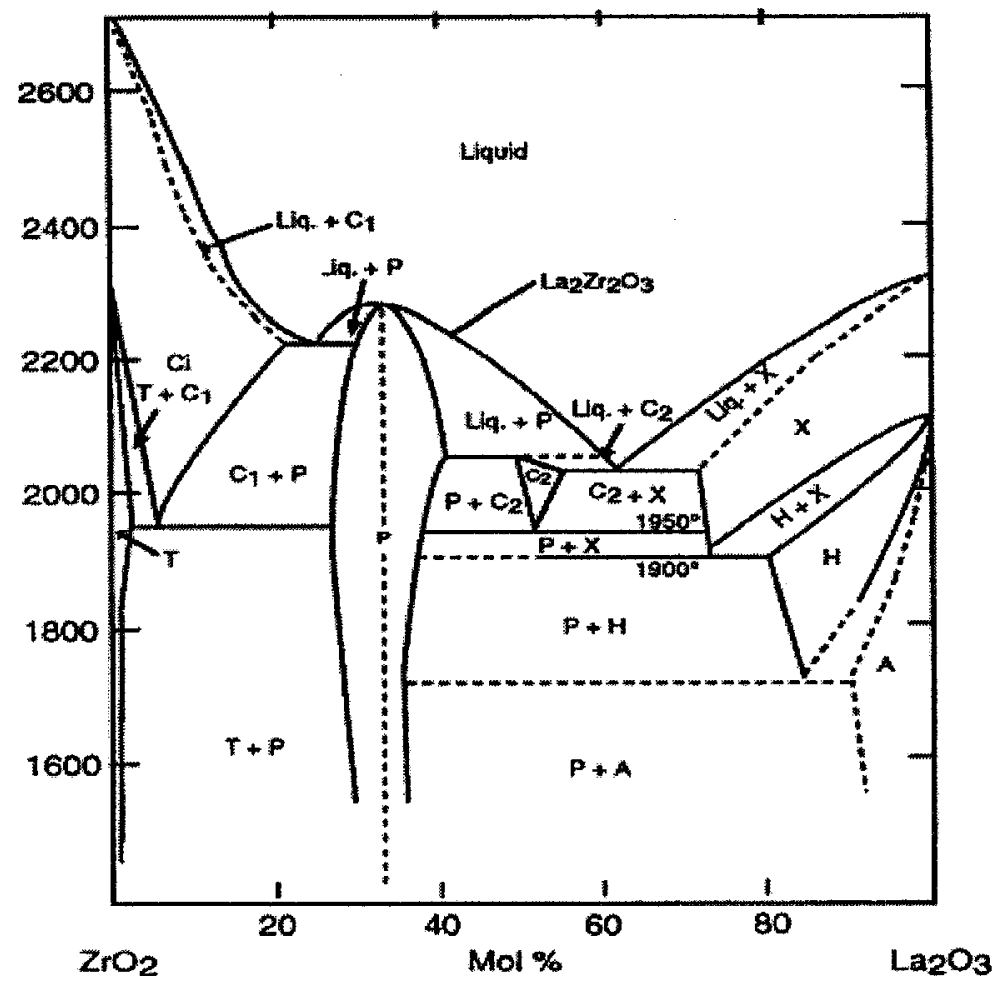

Figure $41 \mathrm{ZrO}_{2}-\mathrm{LaO}_{2}$ phase diagram [96] exhibits stable pyrochlore structure at high temperatures. 
Figure 41 is the $\mathrm{ZrO}_{2}-\mathrm{LaO}_{2}$ phase diagram [96]. It can be seen that the stoichiometric $\mathrm{La}_{2} \mathrm{Zr}_{2} \mathrm{O}_{7}$ has a stable pyrochlore structure up to the melting temperature of $2300^{\circ} \mathrm{C}$. This characteristic of the lanthanum zirconate makes it the most promising material as TBC.

One of the basic requirements for thermal barrier coatings is low intrinsic thermal conductivity, which is associated with the increasing complexity of the crystallographic structure and differences in atomic weight and ionic radius inside the unit cell $[69,72]$. Compared with the cubic fluorite structure of YSZ, the pyrochlore structure is similar, but with one missing oxygen atom and a large number of displaced oxygen atoms within a unit cell. Therefore, the pyrochlore crystal can be considered as an ordered, highly defective fluorite solid solution with reduced symmetry and more complicated structure. As such, it exhibits reduced intrinsic thermal conductivity. Experimental study showed that at $1000^{\circ} \mathrm{C}$, the thermal conductivities are $1.5 \sim 1.6 \mathrm{~W} / \mathrm{m} \cdot \mathrm{K}$ and $1.2 \sim 1.3 \mathrm{~W} / \mathrm{m} \cdot \mathrm{K}$ for dense $\mathrm{La}_{2} \mathrm{Zr}_{2} \mathrm{O}_{7}$ and $\mathrm{Nd}_{2} \mathrm{Zr}_{2} \mathrm{O}_{7}$, respectively $[95,97]$. At $700^{\circ} \mathrm{C}$, the thermal conductivity values range from 1.5 to $1.6 \mathrm{~W} / \mathrm{m} \cdot \mathrm{K}$ for dense $\mathrm{Gd}_{2} \mathrm{Zr}_{2} \mathrm{O}_{7}, \mathrm{Nd}_{2} \mathrm{Zr}_{2} \mathrm{O}_{7}$ and $\mathrm{Sm}_{2} \mathrm{Zr}_{2} \mathrm{O}_{7}$ while the thermal conductivity of dense $7 \mathrm{YSZ}$ is $2.3 \mathrm{~W} / \mathrm{m} \cdot \mathrm{K}$. Figure 42 illustrates the thermal conductivities of some pyrochlore compositions compared with that of YSZ. Lanthanum zirconate and neodymium zirconate as well as gadolinium zirconate exhibit lower thermal conductivities than YSZ.

Among the zirconates examined, lanthanum and zirconium have similar vapor pressure; this makes vapor deposition easier [96] than for the doped zirconia. In addition, atomistic simulation indicated that the rare earth zirconates have higher oxygen-anion Frenkel pair energies than that of YSZ and therefore it requires higher activation energy for oxygen to 
migrate in zirconate $[94,98,99]$. This characteristic of reduced oxygen transparency of lanthanum zirconate provides better bond coat oxidation resistance than YSZ could offer $[100]$.

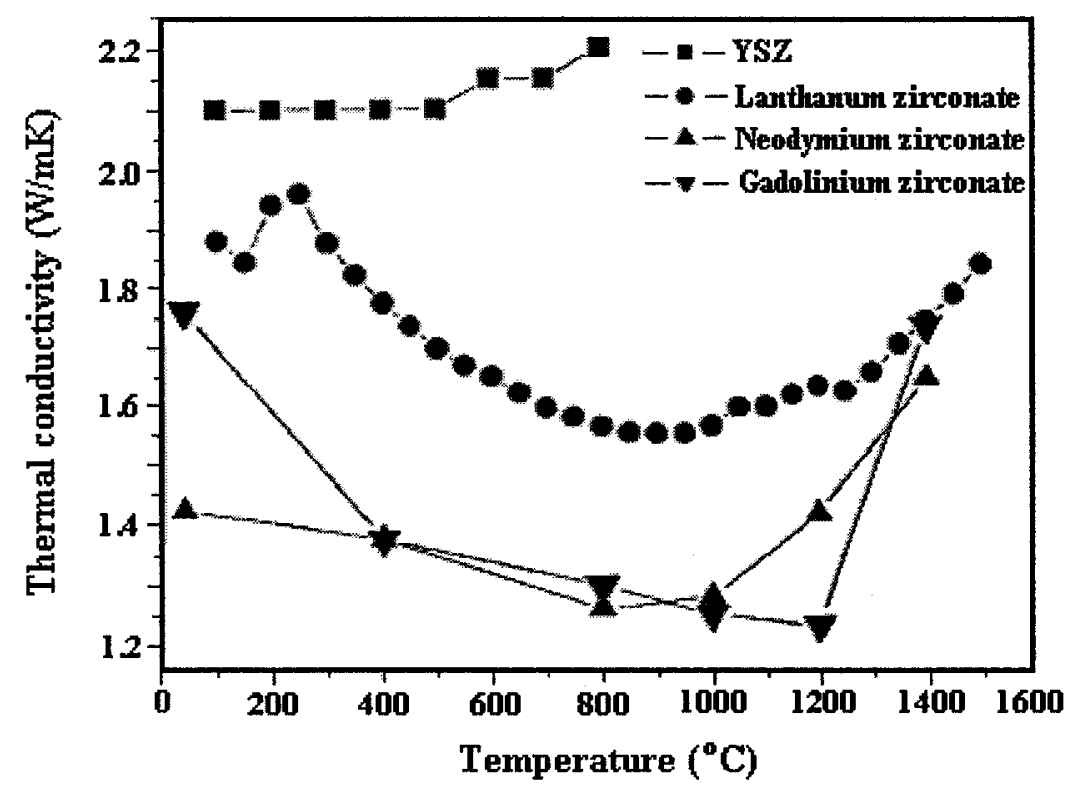

Figure 42 Thermal conductivities of some pyrochlore compositions (compared to bulk YSZ) [97]

However, the lower coefficient of thermal expansion (CTE) of the rare earth zirconates could present challenges for their potential application as TBC since the lower CTE, relative to bond coat and superalloy substrate, may lead to higher thermal stresses during thermal cycling. On the other hand, the Young's moduli of the zirconates are about $15 \%$ lower and could compensate for the effect of the higher mismatch in CTE and alleviate the stresses. The values of CTE as well as Young's moduli for selected zirconates and YSZ are given in Table 3. 
Table 3 Thermal expansion coefficients and Young's moduli of various zirconates

\begin{tabular}{|c|c|c|}
\hline Material & Thermal expansion coefficient $\left(\mathrm{x} 10^{-6} \mathrm{~K}^{-1}\right)$ & Young's modulus (GPa) \\
\hline $\mathrm{Gd}_{2} \mathrm{Zr}_{2} \mathrm{O}_{7}$ & $8.1 \sim 10.5$ at $200 \sim 1000^{\circ} \mathrm{C}[95]$ & $205[99]$ \\
\hline $\mathrm{Eu}_{2} \mathrm{Zr}_{2} \mathrm{O}_{7}$ & $10.3 \sim 10.6$ at $200 \sim 1000^{\circ} \mathrm{C}[95]$ & $219[99]$ \\
\hline $\mathrm{Nd}_{2} \mathrm{Zr}_{2} \mathrm{O}_{7}$ & $9.0 \sim 9.7$ at $200 \sim 1000^{\circ} \mathrm{C}[95]$ & $175 \pm 1[101]$ \\
\hline $\mathrm{La}_{2} \mathrm{Zr}_{2} \mathrm{O}_{7}$ & $8.1 \sim 9.1$ at $200 \sim 1000^{\circ} \mathrm{C}[95]$ & $231[99]$ \\
\hline $\mathrm{Sm}_{2} \mathrm{Zr}_{2} \mathrm{O}_{7}$ & $10.8[98]$ & $250 / 210 \pm 10[102]$ \\
\hline $7 \mathrm{YSZ}$ & 11.5 at $200 \sim 1000^{\circ} \mathrm{C}[102]$ & \\
\hline
\end{tabular}

Other research indicates that the pyrochlore zirconates readily form $\beta$-alumina or perovskite between the TBC and TGO and therefore are not thermodynamically compatible with MCrAlY bond coat system [29]. Further developments are required in order to fully explore the potential of the zirconate based ceramic materials and to identify a more suitable bond coat if zirconates are to be used on superalloy substrates as TBC.

Another ceramic material, the $20 \mathrm{~mol} \%$ yttria stabilized hafnia $\left(\mathrm{HfO}_{2}-\mathrm{Y}_{2} \mathrm{O}_{3}\right)$, was also found to have low thermal conductivity and become a potential TBC candidate [102]. The durability test for this material showed that the phase stability was improved at a temperature of $1650^{\circ} \mathrm{C}$ with the optimized composition. Further investigations on other thermal and mechanical properties of the $\mathrm{HfO}_{2}-\mathrm{Y}_{2} \mathrm{O}_{3}$ ceramics are required. 


\subsection{Methods to Reduce Thermal Radiation through TBCS}

As summarized in the early section, with increase in operating temperatures, thermal radiation will play an important role in heat transfer through TBC. In order to effectively reduce thermal radiation transport through $\mathrm{TBC}$ systems, research has been carried out with emphasis on increasing the photon scattering within the coating and increasing coating's reflectivity. A layered TBC structure, produced using EB-PVD, was able to reduce thermal radiation by increasing scattering defects such as boundaries between multiple layers [104]. Increasing the number of scattering defects such as microcracks and pores within the coating has also been reported as another efficient way of reducing thermal radiation [91]. A new multiple layered coating structure was designed in which highly reflective metallic layers are embedded within the ceramic coatings to reduce the radiation heat transport [105]. A mathematical simulation on the multiple layered coating structure (Figure 43 (a)) concluded that there would be a $12-24 \%$ reduction in the net heat flux (Figure 43 (b)) for a ceramic coating containing a single metallic layer as compared to a ceramic coating without a reflective metallic layer. The use of metal reflective layers in a TBC may, however, be problematic due to the thermal expansion coefficient mismatch between the metallic and ceramic materials, poor adhesion of the ceramic material to the metal, and high thermal conductivity inherent to metals.

To increase the hemispherical reflectance of the coating, Wolfe and Singh et al. [45] have presented a modified multiple layered structure with increased hemispherical reflectance by periodically interrupting the incoming vapor flux during electron beam physical vapor depositing process to vary the densities of alternating layers. The variation in densities 
between layers results in different refractive indices and therefore the reflection of radiation can be enhanced, as shown in Figure 44.

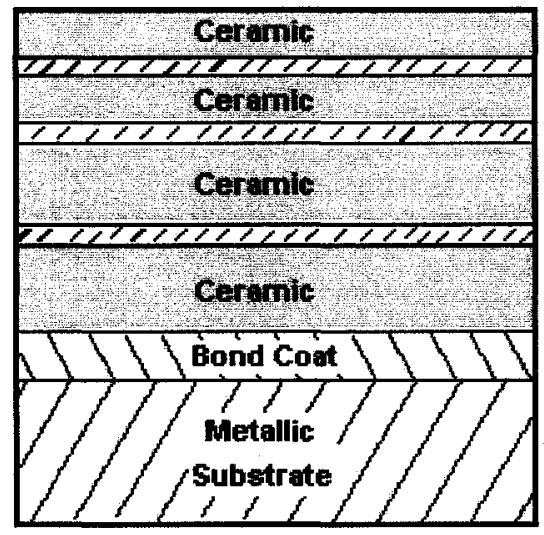

The shade part between ceramic (a) layers are metallic layers

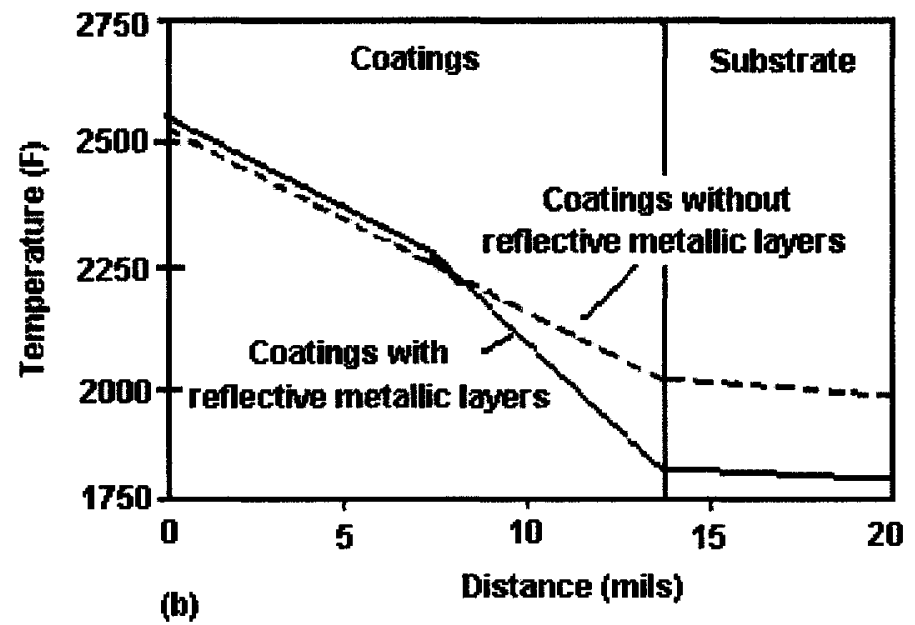

Figure 43 (a) Ceramic coating having metallic reflective layers; and (b) Temperature vs. distance from an exposed surface [105].

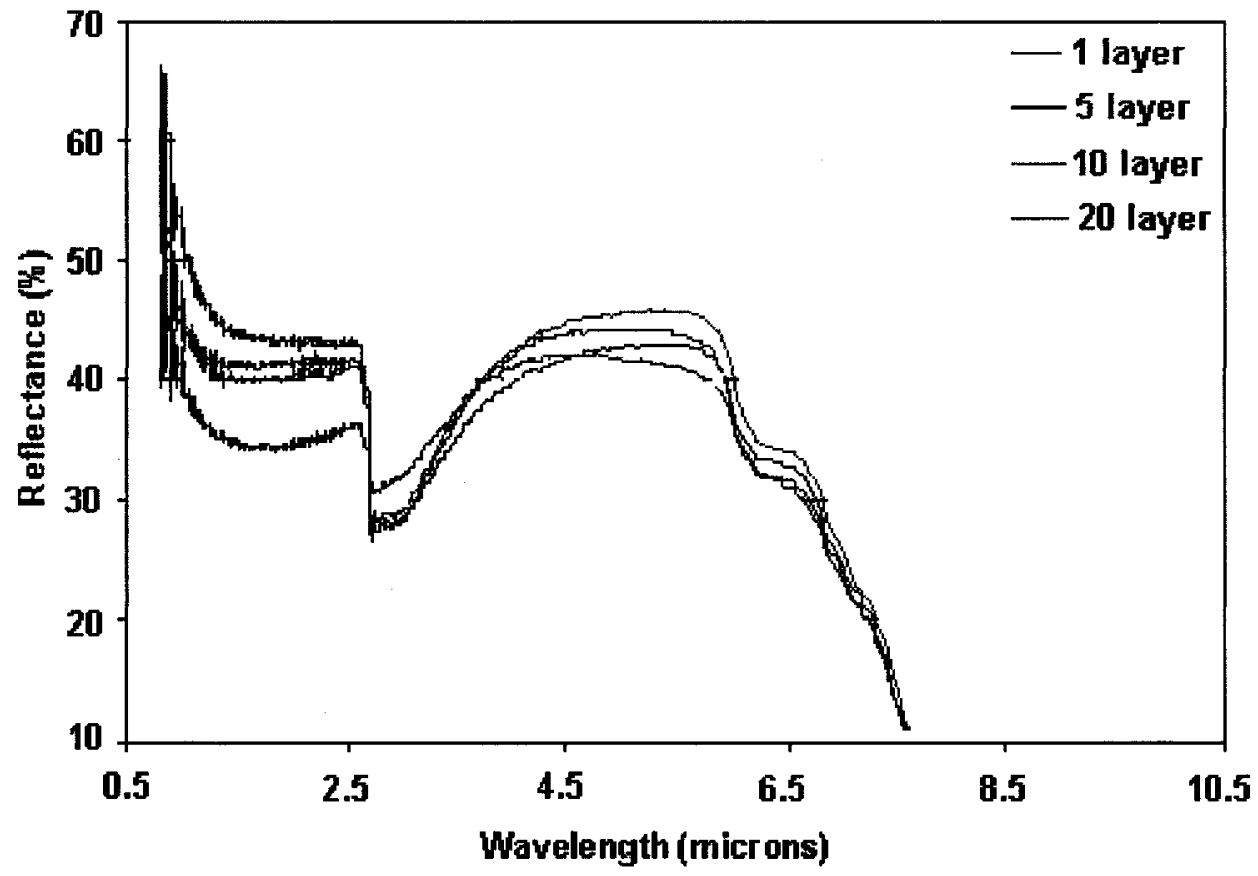

Figure 44 Hemispherical reflectance of 8 YSZ/PtAl/MAR-M247 and effect of layering after 20 hours at $950^{\circ} \mathrm{C}$ [45]. 
Another technique used to produce multiple layered coatings with high reflectance is to incorporate two or more ceramic materials with different chemical compositions; for example, $8 \mathrm{YSZ}$ with high refractive index material and $\mathrm{Al}_{2} \mathrm{O}_{3}$ with low refractive index material can be used to create alternating layers with different refractive indices. The multiple layered structure with alternating $400 \mathrm{~nm} 8 \mathrm{YSZ}$ and $100 \mathrm{~nm} \mathrm{Al}_{2} \mathrm{O}_{3}$ is shown in Figure 45.

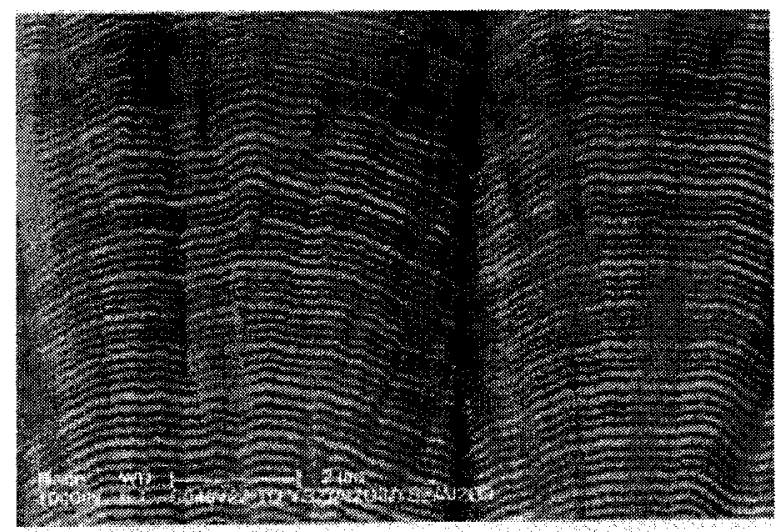

Figure 45 Multiple layered $8 \mathrm{YSZ} / \mathrm{Al}_{2} \mathrm{O}_{3}$ structure with increased hemispherical reflectance [45].

The hemispherical reflectance to radiation at $1 \mu \mathrm{m}$ wavelength was increased from $35 \%$ (single layer) to $45 \%$ (20 layers), as shown in Figure 46 . However, to effectively reflect broadband radiation, optimized design of the individual layer is required. The authors have designed a novel high reflectance multiple layered structure with alternating high and low refractive index for each layer, which covers the wavelength range where currently used ceramic materials are transparent. All these layers use ceramic materials with low thermal conductivities [106, 107]. The detailed design and its mathematical model will be elucidated in a later section. 


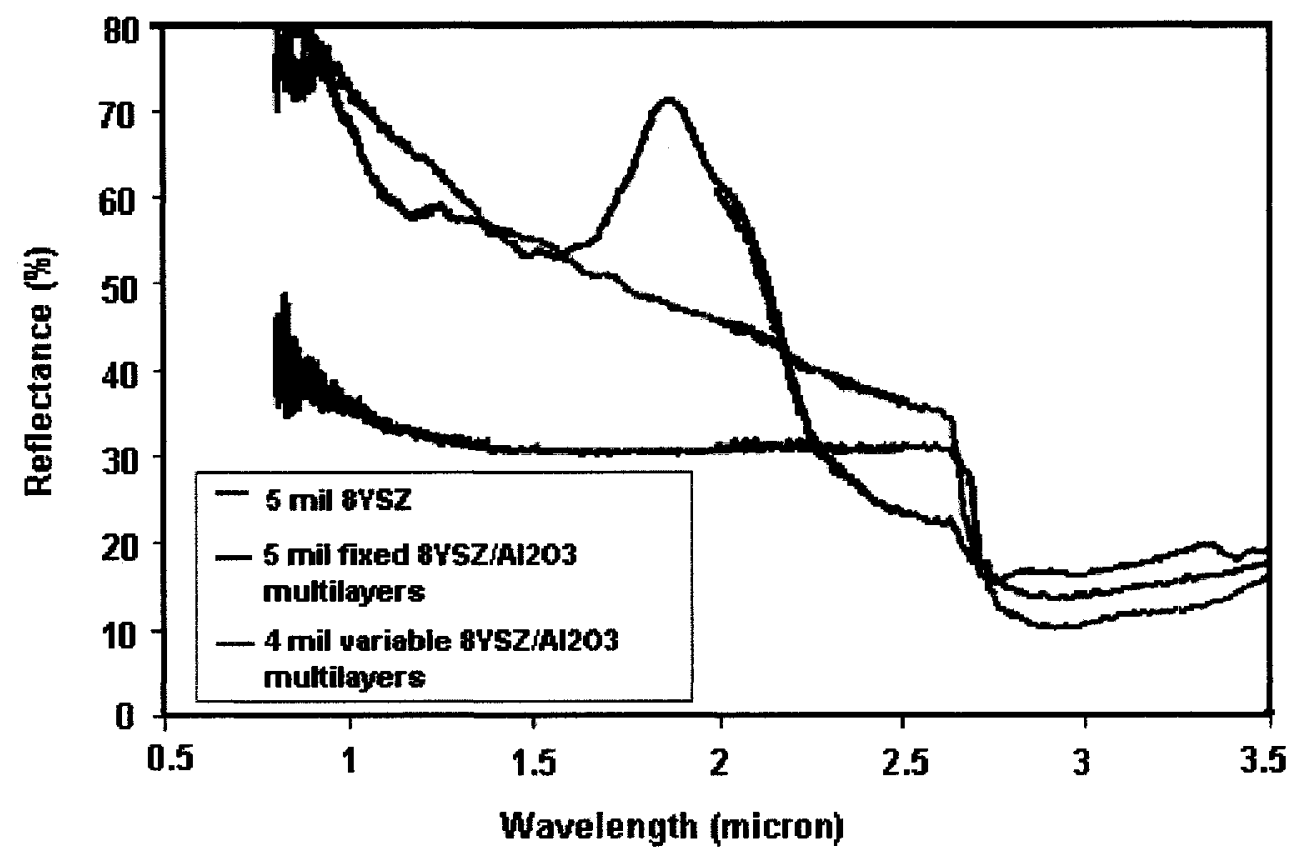

Figure 46 Multilayered TBC increases IR reflectance with fixed and variable spacing [45].

Based on the literature review carried out, the present research will focus on the reduction of both thermal conduction and thermal radiation. More specifically, fabrication and characterization of a series of metal oxide co-doped $7 \mathrm{wt} \%$ yttria stabilized zirconia (7YSZ) materials will be conducted first. A semi-empirical thermal conductivity model based on thermal conductivity theory and experimental results will be established to provide guidance on future selection of TBC materials with low intrinsic thermal conductivities. At the same time multiple layered coatings with high reflectance to thermal radiation will be proposed and a novel TBC structure combining multiple layered stacks with high reflectance to thermal radiation and a single layered coating with low thermal conductivity will be presented. Furthermore, the temperature distributions within 
such structures are to be calculated using energy equations and radiation transfer equations. The outcome of these will provide a multiple layered thermal barrier coating structure with reduced thermal conductivity and thermal radiation heat transport. 


\section{Materials Selection and Experiments}

It has been well documented that the addition of metal oxide to zirconia creates point defects, such as oxygen vacancies and substitution of host atoms. These point defects increase phonon scattering centres and thus result in a decrease in thermal conductivity, as summarized in the literature review.

According to thermal conductivity theory, the scattering effect from each point defect is associated with the type of point defect, oxygen vacancy or substitution of the host atom, and the characteristics of the dopant cations such as mass, ionic radius, valence. For the single metal oxide doped binary zirconia system with trivalent dopants, $\mathrm{Y}^{3+}, \mathrm{Sr}^{3+}, \mathrm{Yb}^{3+}$, it has been experimentally confirmed that thermal conductivity decreases with the increase in ionic radius of the dopant cations $[1,30]$. Unfortunately, the phase of the single metal oxide doped binary system is not always stable at high temperatures, especially for binary systems with larger ionic radius dopant cations where the maximum temperature for phase stability is much lower than that of yttria or ytterbia doped zirconia systems.

On the other side, compared to the binary system, the multiple metal oxide co-doped zirconia system exhibits very promising properties, i.e., more stable phase at high temperatures [29] and lower thermal conductivity $[5,88]$. These benefits make the multiple component doped zirconia very attractive. However, all the published experimental data on thermal conductivity has been based on plasma sprayed or EB-PVD coatings where the uncertainty in porosity may influence the measurements. Additionally, information on how the dopant cations affect thermal conductivity of the 
bulk multiple component doped zirconia is scarce. To understand how the thermal conductivity changes as a function of mass, ionic radius, valence as well as the concentration of the dopants in zirconia based ceramics without the influence of the porosity, and to provide theoretical guidance for selecting TBC materials, a series of ternary metal oxide co-doped zirconia systems with yttria as stabilizer were investigated in this study.

\subsection{Material Considerations}

Table 4 lists the dopant oxides used in this study. The basis for these selections is detailed as follows.

Table 4 Parameters of selected metal oxides used as additions to 7YSZ

\begin{tabular}{|c|c|c|c|}
\hline Dopant & Dopant cation & Atomic mass of the cation & Ionic radius of the cation $(\mathrm{nm})$ \\
\hline $\mathrm{Y}_{2} \mathrm{O}_{3}$ & $\mathrm{Y}^{3+}$ & 88.91 & 0.089 \\
\hline $\mathrm{Ta}_{2} \mathrm{O}_{5}$ & $\mathrm{Ta}^{5+}$ & 180.95 & 0.068 \\
\hline $\mathrm{Nb}_{2} \mathrm{O}_{5}$ & $\mathrm{Nb}^{5+}$ & 92.91 & 0.069 \\
\hline $\mathrm{Cr}_{2} \mathrm{O}_{3}$ & $\mathrm{Cr}^{3+}$ & 52.00 & 0.063 \\
\hline $\mathrm{Sc}_{2} \mathrm{O}_{3}$ & $\mathrm{Sc}^{3+}$ & 44.96 & 0.072 \\
\hline $\mathrm{Yb}_{2} \mathrm{O}_{3}$ & $\mathrm{Yb}^{3+}$ & 173.04 & 0.086 \\
\hline $\mathrm{CeO}_{2}$ & $\mathrm{Ce}^{4+}$ & 140.11 & 0.092 \\
\hline
\end{tabular}

To investigate the thermal conductivity changes independent of the influence of oxygen 
vacancies, tetravalent oxide, cerium oxide, was added to 7YSZ. When a cerium ion replaces a zirconium ion, there are no extra oxygen vacancies generated. Thus the reduction, if any, in thermal conductivity can only be caused by differences in the masses and ionic radii between the cerium cation and the substituted zirconium cation.

The pentavalent oxides, tantalum pentoxide $\mathrm{Ta}_{2} \mathrm{O}_{5}$ and niobium pentoxide $\mathrm{Nb}_{2} \mathrm{O}_{5}$, are selected with the objective of adding two oxides in the same amounts as that of $\mathrm{Y}^{3+}$ in 7YSZ to eliminate the influence of oxygen vacancies. The pentavalent tantalum and pentavalent niobium have similar ionic radii, however, different ionic masses. The ionic radii of tantalum and niobium are smaller than that of zirconium, thus the replacement of a zirconium ion by either a tantalum ion or a niobium ion will cause crystal distortion and result in changes in bonding force. In addition, the mass of a niobium ion is close to that of a zirconium ion, whereas the mass of a tantalum is double that of zirconium. This selection is based on the hypothesis that tantala dopant should have more influence on thermal conductivity than niobia due to the mass differences between the dopant and the host atom. In solid solution, all the dopant cations including both trivalent yttrium ions and pentavalent ions will take over the host zirconium ions. Due to the electric charge compensation, oxygen vacancies created by trivalent yttrium annihilate when pentavalent ions are added.

To investigate how the thermal conductivity changes with ionic radii of the dopant cation while ensuring that the valences and masses for the selected dopants are the same, chromium oxide $\mathrm{Cr}_{2} \mathrm{O}_{3}$ and scandium oxide $\mathrm{Sc}_{2} \mathrm{O}_{3}$ are selected. Since $\mathrm{Cr}^{3+}$ and $\mathrm{Sc}^{3+}$ have similar masses, and the same valences but different ionic radii, the chromium oxide and 
scandium oxide could provide information on the influence of ionic radius of dopant on various properties of doped YSZ. While trivalent dopants $\mathrm{Cr}^{3+}$ and $\mathrm{Sc}^{3+}$ have smaller valences than that of zirconium, the defects types will be substitutional $\mathrm{M}^{3+}$ replacing $\mathrm{Zr}^{4+}$ and extra oxygen vacancy sites.

\subsection{Fabrication of Co-doped Zirconia-based Bulk Materials}

\subsubsection{Experimental Considerations}

The purpose of this experiment is to produce dense, crack-free, and chemically homogeneous bulk ceramic materials using a sintering process. Sintering is a process of solid-state reaction and densification due to the atomic diffusion, accompanying a decrease in the free energy of the system [108]. The actual occurrence of sintering is accomplished by the transport of mass, and the movement of different types of defects. During sintering, the compacted bodies will generally experience three mechanisms of diffusion: surface diffusion at low temperature, resulting in neck growth between particles; grain boundary diffusion at elevated temperatures, capable of achieving relative densities of $90 \%$ to $95 \%$; and lattice diffusion during the final stage, caused by the movement of point defects through the bulk of the lattice, controlling the grain growth and the chemical homogeneity of the final bulk material.

The rates of sintering are dependent on the number of contact points between different particles, the diffusion distances and the activation energies of the diffusion mechanisms described above, and sintering temperatures. Ceramics with ionic bonds have very high activation energies for lattice diffusion; to increase the rate of sintering, the sintering 
temperatures required are therefore very high. However, it has been well documented that smaller particle size, narrow particle size distributions and the consolidation of the powders prior to sintering will reduce the activation energies, and further reduce sintering temperatures or increase the sintering rate and reduce sintering time. Fine powders will have higher surface energies, a greater number of contact points between particles, and better physical and chemical uniformity; and compaction will decrease diffusion distances.

Considering all the factors affecting the sintering behavior, the following process steps were developed.

1) Powder blending and comminuting were used to mix multiple powders uniformly in composition and to obtain finer powders and proper particle size distributions.

2) Powder consolidation was used to form high density green bodies so that the diffusion distance would be shortened and mass transport rate increased.

3) Green bodies were heated isothermally for a period of time at high temperatures so that the chemical homogeneity and densification could occur by diffusion.

The procedure for the fabrication of bulk zirconia with metal oxide dopants is shown in Figure 47. The sintering is a diffusional process that proceeds at relatively high temperatures, usually between $1 / 2$ and $3 / 4$ of the melting temperature of the ceramic [109]. The sintering temperature and activation energy for lattice diffusion are determined based on published literatures [110]. For $\mathrm{ZrO}_{2}$ ceramic powders with particle sizes of $40 \sim 260 \mu \mathrm{m}$, the grain boundary diffusion and densification of the compact body occurred 
within the temperature range of $1100-1300^{\circ} \mathrm{C}$, and the lattice diffusion and $\mathrm{ZrO}_{2}+\mathrm{Y}_{2} \mathrm{O}_{3}$ reaction took place within the temperature range of $1300 \sim 1500^{\circ} \mathrm{C}$. Thus, in this study, the isothermal sintering temperature was selected to be $1500^{\circ} \mathrm{C}$ to ensure the activation of lattice diffusion and reaction between 7YSZ and dopants.

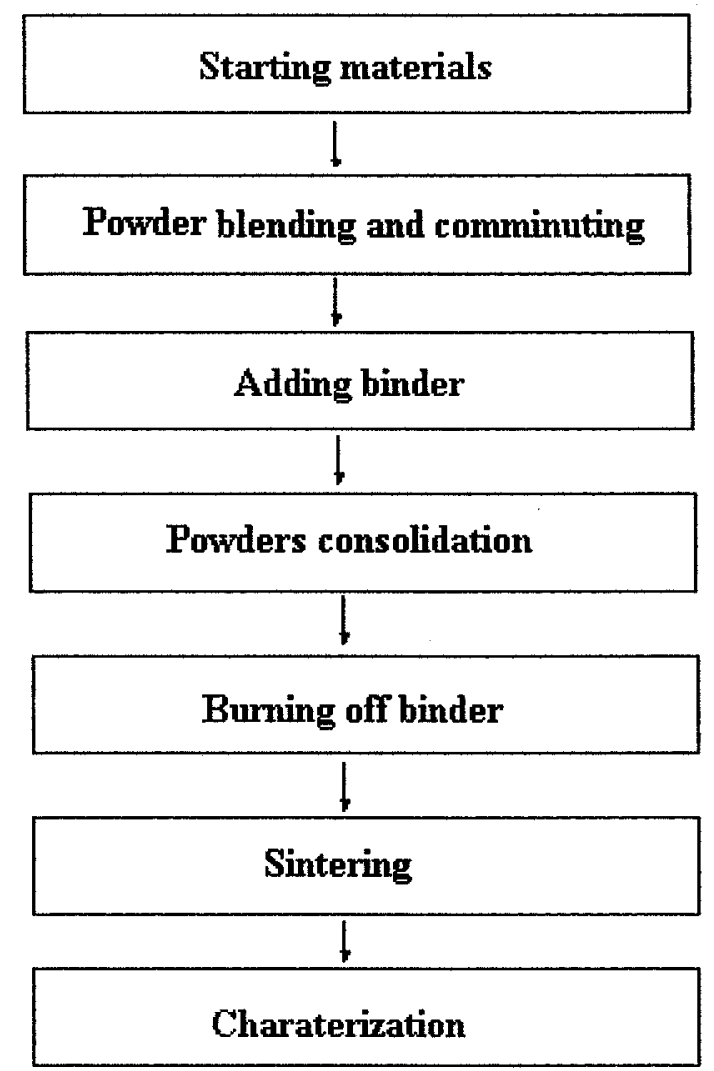

Figure 47 Flow chart for the fabrication of bulk materials with doped metal oxides.

The sintering time required is determined based on the Fick's second law, the Arrhenius relation, the concentration of dopants, and the particle size so that the sintered bulk materials are chemically homogeneous. According to the Fick's second law, in one dimensional model along $x$ direction, the concentration $C$ as a function of time $t$ is given by [111]: 


$$
\frac{d C}{d t}=D \cdot\left(\frac{d^{2} C}{d x^{2}}\right)
$$

where $D$ is the diffusion coefficient, a material property for characterizing the rate of diffusive mass transport and is given by the Arrhenius relation:

$D=D_{0} \cdot e^{\frac{Q}{R T}}$

(Eq. 53)

where $Q$ is the activation energy per mol. Solving (Eq. 52), the concentration at a distance $x$ from the surface at the sintering time $t$ is obtained as:

$$
C=\left(\frac{C_{0}}{\pi D t}\right)^{\frac{1}{2}} \cdot e^{-\frac{x^{2}}{4 D t}}
$$

For zirconia-based ceramics, the diffusion coefficient $\mathrm{D}$ of $\mathrm{Zr}$ cations in $\mathrm{CaO}-\mathrm{ZrO}$ system was reported to be $D=10^{-12} \mathrm{~cm}^{2} / \mathrm{s}$ [112] at $1500^{\circ} \mathrm{C}$. Assume a diffusion depth $x_{P}=35 \mu m$ (considering the average particle size) and a concentration of dopants of $50 \%$ at $x_{p}=35 \mu \mathrm{m}$, the sintering time was estimated to be about $100 \mathrm{hrs}$. In the experiment conducted in this study, $120 \mathrm{hrs}$ sintering time was selected to account for the differences in ionic radii among various dopants.

\subsubsection{Starting Materials}

Starting materials were high purity crystalline 7YSZ obtained from Praxair Surface Technology. The powder specifications for 7YSZ are given in Table 5. 
Table 5 Powder specifications for 7YSZ

\begin{tabular}{|l|l|}
\hline Manufacturer & Praxair Surface Technology \\
\hline Powder Size (mesh) & 325 \\
\hline Primary particle size $(\mu \mathrm{m})$ & $1 \sim 15$ \\
\hline Composition & $3.945 \mathrm{~mol}^{\circ} \mathrm{Y}_{2} \mathrm{O}_{3}-\mathrm{ZrO}_{2}$ \\
\hline Purity (\%) & 99.9 \\
\hline
\end{tabular}

Table 6 Powder specifications for dopants

\begin{tabular}{|l|l|l|}
\hline Dopants & \multicolumn{2}{|l|}{ Powder specifications } \\
\hline $\mathrm{Ta}_{2} \mathrm{O}_{5}$ & Manufacturer & Metall Rare Earth Limited \\
\cline { 2 - 3 } & Primary particle size $(\mu \mathrm{m})$ & $<<1$ \\
\cline { 2 - 3 } & Purity (\%) & 99.9 \\
\hline $\mathrm{Nb}_{2} \mathrm{O}_{5}$ & Manufacturer & Sigma-Aldrich \\
\cline { 2 - 3 } & Primary particle size $(\mu \mathrm{m})$ & $<<1$ \\
\cline { 2 - 3 } & Purity (\%) & 99.9 \\
\hline $\mathrm{Cr}_{2} \mathrm{O}_{3}$ & Manufacturer & Sigma-Aldrich \\
\cline { 2 - 3 } & Primary particle size $(\mu \mathrm{m})$ & $<<1$ \\
\cline { 2 - 3 } & Purity (\%) & 99.9 \\
\hline $\mathrm{Sc}_{2} \mathrm{O}_{3}$ & Manufacturer & Metall Rare Earth Limited \\
\cline { 2 - 3 } & Primary particle size $(\mu \mathrm{m})$ & $1 \sim 10$ \\
\cline { 2 - 3 } & Purity (\%) & 99.9 \\
\hline $\mathrm{Yb}_{2} \mathrm{O}_{3}$ & Manufacturer & Metall Rare Earth Limited \\
\cline { 2 - 3 } & Primary particle size $(\mu \mathrm{m})$ & $1 \sim 10$ \\
\cline { 2 - 3 } & Purity (\%) & 99.9 \\
\hline $\mathrm{CeO}_{2}$ & Manufacturer & Metall Rare Earth Limited \\
\cline { 2 - 3 } & Primary particle size $(\mu \mathrm{m})$ & $1 \sim 10$ \\
\cline { 2 - 3 } & Purity (\%) & 99.9 \\
\hline
\end{tabular}

The tantalum oxide $\mathrm{Ta}_{2} \mathrm{O}_{5}$, scandium oxide $\mathrm{Sc}_{2} \mathrm{O}_{3}$, cerium oxide $\mathrm{CeO}_{2}$ and ytterbium oxide $\mathrm{Yb}_{2} \mathrm{O}_{3}$ were obtained from Metall Rare Earth Limited. The powders of niobium 
oxide $\mathrm{Nb}_{2} \mathrm{O}_{5}$ and chromium oxide $\mathrm{Cr}_{2} \mathrm{O}_{3}$ are from Sigma-Aldrich. The dopant powder specifications are listed in Table 6.

These metal oxide powders were added to 7YSZ powders according to the compositions of 7YSZ - 3.945 mol\% $\mathrm{Ta}_{2} \mathrm{O}_{5}$ (3.9TaYSZ), $7 \mathrm{YSZ}-3.945 \mathrm{~mol} \% \mathrm{Nb}_{2} \mathrm{O}_{5}(3.9 \mathrm{NbYSZ})$, 7YSZ -5 mol\% $\mathrm{CrO}_{1.5}$ (5CrYSZ), 7YSZ -5 mol\% $\mathrm{ScO}_{1.5}$ (5ScYSZ), $7 \mathrm{YSZ}$-5 mol\% $\mathrm{CeO}_{2}$ (5CeYSZ), and 7YSZ -5 mol\% $\mathrm{YbO}_{1.5}$ (5YbYSZ), respectively. The compositions of these metal oxide dopants as well as the content of $Y$ cation are shown in Table 7.

Table 7 Compositions of the dopant cations in doped 7YSZ systems

\begin{tabular}{|c|c|c|c|c|}
\hline \multirow{2}{*}{ Sample ID } & \multirow{2}{*}{$\begin{array}{c}\text { Co-dopant } \\
\text { cation }\end{array}$} & \multicolumn{2}{|c|}{ Content of co-dopant cation } & \multirow{2}{*}{$\begin{array}{c}\text { Content of Y } \\
(\mathrm{mol} \%)\end{array}$} \\
\hline & & $(w t \%)$ & $(\mathrm{mol} \%)$ & \\
\hline 3.9TaYSZ & $\mathrm{Ta}^{5+}$ & 12.03 & 7.05 & 7.06 \\
\hline 3.9NbYSZ & $\mathrm{Nb}^{5+}$ & 7.60 & 7.05 & 7.06 \\
\hline $5 \mathrm{CrYSZ}$ & $\mathrm{Cr}^{3+}$ & 2.98 & 4.71 & 7.23 \\
\hline $5 S c Y S Z$ & $\mathrm{Sc}^{3+}$ & 2.71 & 4.71 & 7.23 \\
\hline $5 \mathrm{YbYSZ}$ & $\mathrm{Yb}^{3+}$ & 7.37 & 4.71 & 7.23 \\
\hline $5 \mathrm{CeYSZ}$ & $\mathrm{Ce}^{4+}$ & 6.50 & 4.71 & 7.23 \\
\hline
\end{tabular}

\subsubsection{Powder Blending and Comminuting}

The powder blending and comminuting are performed using a ball-milling method. Since the rate of milling increases with the density of the balls and inversely as the radius of the balls [113], small, heavy balls were therefore considered when selecting grinding media. Another concern is the contamination from the wear of the grinding. To prevent 
contamination, pure $\mathrm{ZrO}_{2}$ balls were selected. $\mathrm{ZrO}_{2}$ has a higher density of $6.27 \mathrm{~g} / \mathrm{cm}^{3}$ compared with other ceramic materials. The size of each $\mathrm{ZrO}_{2}$ ball was $10 \mathrm{~mm}$ in diameter. A neoprene barrel with quick-sealed, leak proof closures was purchased from United Nuclear, Scientific Equipment \& Supplies. The dimensions of the barrel is $4.5^{\prime \prime}$ in diameter and $4.75 "$ in height. In the experiment, the barrel was filled with $1.9 \mathrm{lb}$ zirconia balls and $1 \mathrm{lb}$ starting powders, and placed on a Turbula T2C mix shaker made by Willy A. Bachofen AG Maschinenfabrik $\mathrm{CH}-4005$ Basel/ Schweiz. The rotation of the shaker was set to its lowest speed of $20 \mathrm{t} / \mathrm{min}$ (turns per minute) to avoid overheat inside the mill due to friction. The grinding cycle was $2 \mathrm{hrs}$ on and $1 \mathrm{hr}$ off. The total blending time is 50 hours. Since the rate of grinding also depends on the initial particle size [113], it is very difficult to comminute the powder further when the particle size reaches $1 \mu \mathrm{m}$. Figure 48 shows the powders morphology after ball milling for different milling times. A Hitachi S-570 scanning electron microscope was used to examine the powder size and morphology.
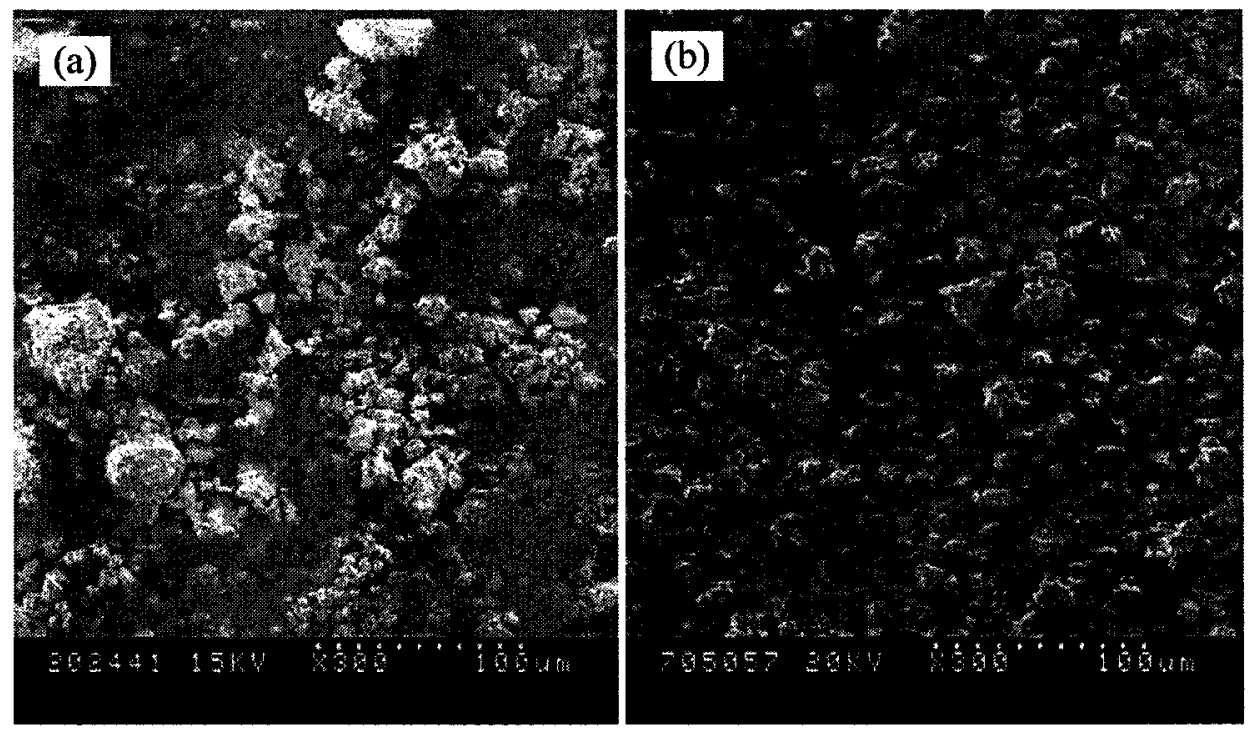

Figure 48 SEM image of powder size after ball milling (a) $20 \mathrm{hrs}$ and (b) $50 \mathrm{hrs}$. 101 


\subsubsection{Powder Consolidation}

The process of powder consolidation is used to form a homogeneous green body with high packing density. The effect of compact pressure on green density and sintering density for microsized $8 Y S Z$ powders has been examined [110]. The results indicated that increasing the compaction pressure beyond $150 \mathrm{MPa}$ did not result in higher sintered density. Thus, in our experiment, the uniaxial pressure of $200 \mathrm{MPa}$ was thought to be sufficient.

All the powders were uniaxially pressed at $200 \mathrm{MPa}$ using cylindrical $25 \mathrm{~mm}$ dies. To prevent cross contamination, each die was used for one specific composite powder. The dies were made from precipitation hardened stainless steel and the surfaces were well polished to reduce friction between the particles and the wall. Before the milled powders were loaded into the die, a small amount of binder was added to provide strength to the green body. After the pressure was applied for 10 minutes, the compacted body was ejected from the die. To minimize friction between the compact and the die wall and surface of the punches, a Teflon release agent was used as lubricant.

\subsubsection{Sintering Process}

The samples were sintered in an air furnace at $1500^{\circ} \mathrm{C}$. When the furnace temperature reached $200^{\circ} \mathrm{C}$, the binder was burned off. The furnace was a Lindberg/Blue BF51333C. It is constructed with rugged firebrick insulation for stability and silicon carbide heating elements to maximize heat transfer. A digital single setpoint controller was used for the temperature control. The equipment is shown in Figure 49. 


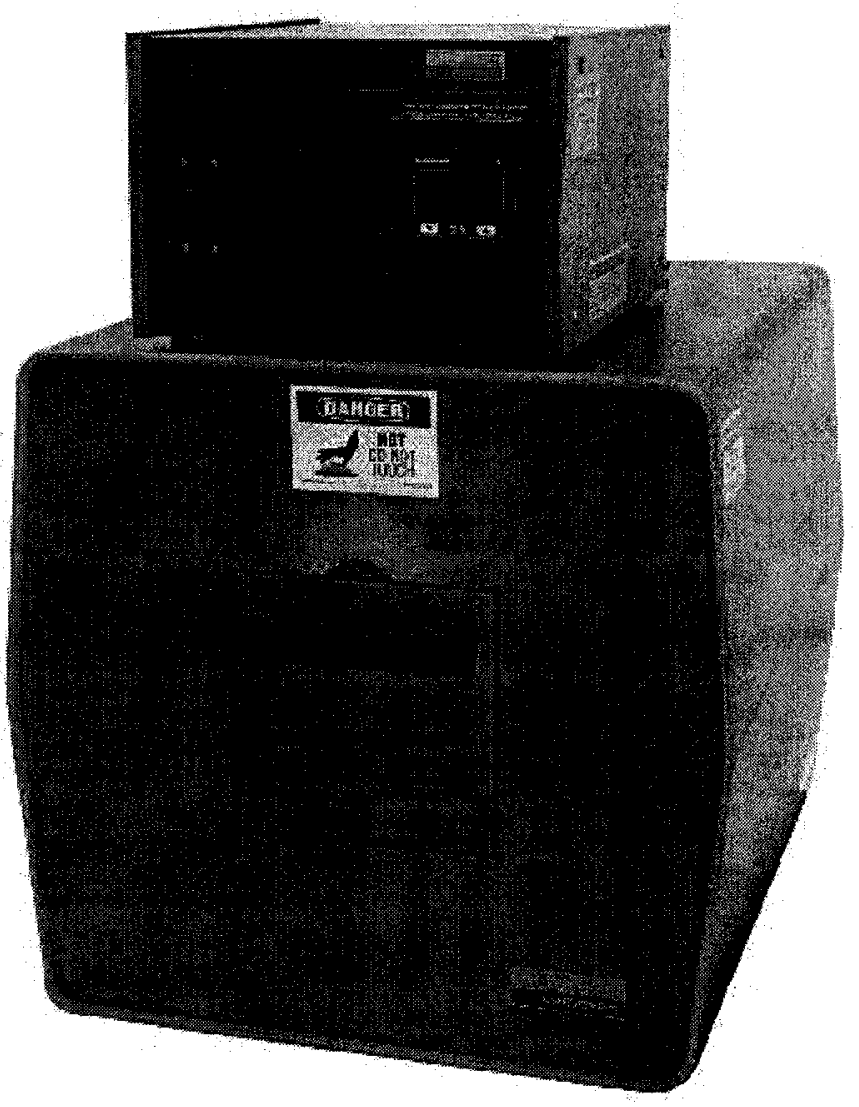

Figure 49 Furnace used for sintering metal oxide co-doped zirconia based ceramics.

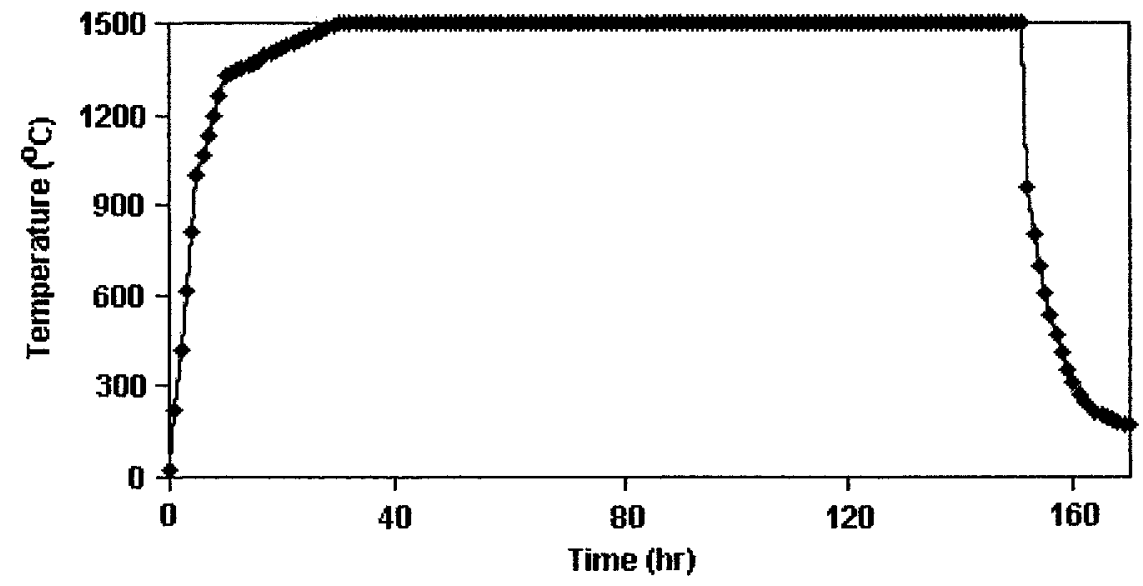

Figure 50 Temperature cycle for the sintering process. The holding time at $1500^{\circ} \mathrm{C}$ is 120 hours. 
Figure 50 shows the temperature cycle for the sintering process. The heating rate was $3 \sim 4^{\circ} \mathrm{C} / \mathrm{min}$ from room temperature to $1000^{\circ} \mathrm{C}$; between $1000^{\circ} \mathrm{C} \sim 1350^{\circ} \mathrm{C}$, the heating rate was reduced to $1 \sim 2^{\circ} \mathrm{C} / \mathrm{min}$; above $1350^{\circ} \mathrm{C}$, the heating rate was less than $1^{\circ} \mathrm{C} / \mathrm{min}$. Holding time at $1500^{\circ} \mathrm{C}$ was 120 hours. After sintering, the samples were furnace cooled. The cooling rate was $13 \sim 14^{\circ} \mathrm{C} / \mathrm{min}$ before the temperature was lowered to $1000^{\circ} \mathrm{C}$; the cooling rate was $4.6 \sim 1.52^{\circ} \mathrm{C} / \mathrm{min}$ before the temperature was dropped to $700^{\circ} \mathrm{C}$ from $1000^{\circ} \mathrm{C}$

104 


\section{Characterization of the Alloyed Powders and the Sintered Materials}

In this chapter, the experimental tools and methods for examining the sintering behavior and properties of the doped zirconia based materials are described. The characterization of the sintered materials included:

1. Density measurement. The density of the sintered material would provide the information on the densification of the sintered samples. This was also an important parameter in calculating the thermal conductivity. The density was measured using Archimedes method [114].

2. Powder characterization. The morphologies of the original powders and the alloyed powders were characterized using scanning electron microscopy (SEM). From the secondary electron images (SEI) obtained from the SEM, the powder sizes and size distributions were examined so that the time for mechanical alloying was determined.

3. Microstructural examinations. To obtain further information on the extent of sintering, the microstructures of the doped samples were examined using scanning electron microscopy (SEM). Both secondary electron images (SEI) and backscattering electron images (BEI) were captured. The SEI was used to examine the sample surface and fraction sections; and the BEI was used to conduct image analysis and elemental composition analysis.

4. Evaluation of volume fraction of porosity. The porosity within the samples will 
affect the mechanical and thermal properties of the sintered materials. To calculate the fraction of porosity, the BEI obtained from SEM and image analyzer were used.

5. Elemental composition analysis. To examine the chemical homogeneity of the sintered materials, quantitative elemental composition analysis was performed using energy dispersive spectroscopy (EDS). Both point analysis and dot mapping were conducted by collecting the specified characteristic $x$-ray of the element of interest.

6. Phase identification. The phase present after sintering in each sample was analyzed using $\mathrm{X}$-ray diffraction (XRD) techniques. In addition, the molar fractions of monoclinic phase were evaluated from the x-ray diffraction patterns using the method proposed by Miller et al [115].

7. Thermal analysis. The study of phase transformations as well as the specific heat evaluation of each sample were performed using differential scanning calorimetry (DSC). The values of the measured specific heat were used for the calculation of thermal conductivities; and the behavior of phase transition at high temperatures could help to determine the high temperature phase.

8. Determination of mechanical properties. The influence of the dopants on the mechanical properties of the sintered materials was examined by measuring elastic modulas using a nano-indentation method.

9. Measurement of thermal diffusivity. The thermal diffusivity of each doped YSZ sample was measured using a laser flash technique so that the thermal conductivity of the sample can be calculated. 
The detailed testing methods and the equipment used are discussed in the following sections.

\subsection{Density Measurement}

According to the Archimedes's theorem, the bulk density of a solid is given as:

$$
\rho_{\text {bulk }}=\frac{m_{d r y} \rho_{\text {liquid }}}{m_{\text {sat }}-m_{\text {susp }}}
$$

where: $\rho_{\text {bulk }}$ is the bulk density of the sample; $m_{d r y}$ is the dry mass of the sample; $\rho_{\text {liquid }}$ is the density of the saturating / suspending liquid; sample; $m_{\text {sat }}$ is the saturated mass of the sample; and $m_{\text {susp }}$ is the suspended mass of the sample. Thus, to obtain the density of the sintered material, data on the dry mass $m_{d r y}$, the saturated mass $m_{s a t}$ and the suspended mass $m_{\text {susp }}$ was required.

All the masses were measured using a digital weighing scale with an accuracy of $0.00001 \mathrm{~g}$. Approximately $1 \mathrm{~g}$ of specimen was used for each measurement. The sintered pellets were weighed for their dry masses first. The samples were then submerged in distilled water. The suspended weight was measured by hanging a saturated pellet from the balance into a beaker of distilled water. Next, the saturated weight was measured by blotting the sample with a paper towel to remove the excess water from the surface. Substituting these measured data and the density of distilled water into (Eq. 55), the sintered density of various metal oxide doped yttria stabilized zirconia bulk materials 
were calculated.

\subsection{Powder Characterization}

The Hitachi S-570 SEM was used to examine the powder sizes and size distributions for both the original powders and the powders after mechanical alloying.

The Hitachi SEM was a microscope with a magnification range of $35 \mathrm{X}$ to $10,000 \mathrm{X}$. This microscope used a tungsten filament thermionic emission gun as its electron beam emission source. When the electron beam emitted from the electron gun was focused onto the surface of the sample in a scanning pattern, interactions between the incoming electrons and the atoms composing the sample produced three types of signals with different energies: secondary electrons, backscattered electrons and characteristic x-rays, as schematically illustrated in Figure 51 [116]. These signals were emitted from the different regions of the specimen and could provide the required information on the materials.

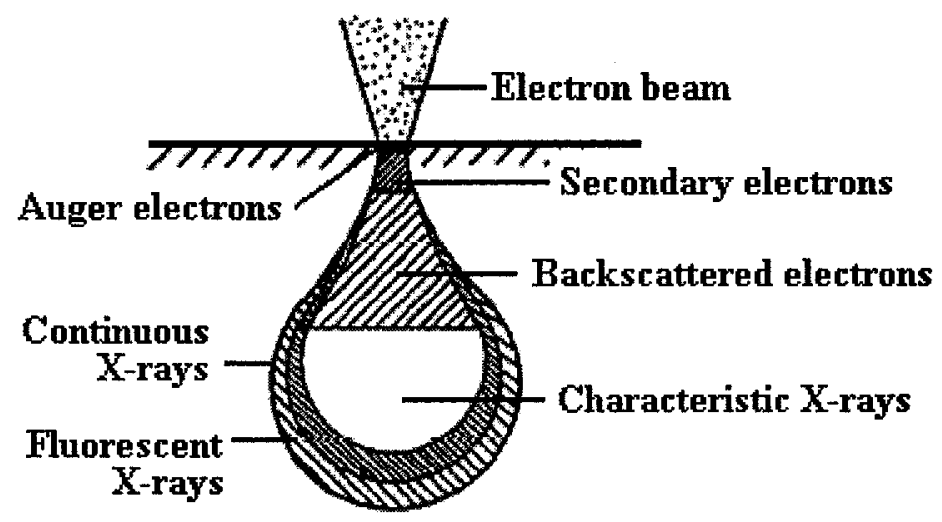

Figure 51 Different types of electrons originating from the interaction volume of the samples [116]. 
The secondary electrons (SE) were caused by inelastic collisions with the atoms in the specimen and generally had a low energy ranging from 0 to several tens of eV. Because of this low energy, only the electrons emitted from a thin layer (in the nm range) could be detected. Since the secondary electrons emitted in all directions could be collected by the secondary detector, an image such as by shadowless illumination could be obtained. The variation in contrast was therefore determined by the sample's topography. Thus, the secondary electron signals were considered to be the most suitable signals for providing information on the specimen's surface topography.

The backscattered electrons (BSE) were caused by elastic collisions between the incoming primary electrons and the nuclei of the sample atoms and had energy as high as that of the incident electrons. Due to their higher energy, the information from the deeper layers (in the range of several 10's of $\mathrm{nm}$ to $100 \mathrm{~nm}$ ) could be obtained. If the specimen is even, the backscattering coefficient of incident electrons is larger for the atom with large atomic numbers and thus resulting in a brighter area in the image. Therefore, two types of information could be obtained from the backscattered electron image: the composition difference and the topography. Regions differing in composition (e.g. grain interior and grain boundary) could therefore be identified. The backscattered electrons were generally detected by a highly sensitive annular type semiconductor detector, which is located above the specimen.

Characteristic $\mathrm{x}$-rays were emitted from the specimen when an electron from the primary beam removed an electron from an inner shell of a specimen atom, an outer shell electron with higher energy decayed and filled the vacancy by releasing an $\mathrm{x}$-ray photon. Since 
the energies of individual shell electrons were determined by the type of elements, the $\mathrm{x}$ ray generated by the electron transition from high-energy shell to low-energy shell was called the characteristic x-ray and could be used for identifying the element. In addition, the intensity of the X-rays is directly associated with weight or atomic fraction of each of those chemical elements. Thus, the x-rays could be used for analyzing the element both qualitatively and quantitatively.

Based on the SEM functions described above, to analyze the powders morphologies, the secondary electron images were enough to provide information on the powder size, shape and size distributions.

Sample preparation: A thin layer of powder particles was firmly attached to a SEM stage using a tape. Then, an air blow gun was to clean the specimen so that no loose particles were left. Since the non-conductive ceramic powder could result in charging effects due to the buildup of a space charge region, which would deflect the incident electron beam and lead to image distortions, a thin layer of conductive carbon was deposited onto the specimen's surface using an E306 Edwards vacuum coater. The layer thickness was about $200 \AA$.

\subsection{Microstructural Examination of the Sintered Samples}

To acquire information on porosity at the surface and inside the sintered samples, grain size and sintering effects, the secondary electron images captured from the surface and fracture sections were obtained using a Hitachi S-570 SEM. To analyze the elemental compositions within the sintered samples and to calculate the volume fraction of porosity 
and grain size, the backscattered electron images, which were used for EDS analysis and image analyzer, respectively, were obtained from the etched/polished surfaces of the samples.

Sample preparation: All the specimens were ultrasonically cleaned and firmly attached to the sample stage. The surfaces were carbon-coated using an E306 Edwards vacuum coater to prevent charging effects due to the non-conductivity of zirconia-based ceramics. For the specimens used for obtaining BEI, the surfaces of the cross sections were polished using diamond-coated polishing films and diamond up to $0.25 \mu \mathrm{m}$; and then etched using $10 \% \mathrm{HF}$ acid for 30 minutes so that the grain boundary could be easily identified.

Finally, the grain sizes were estimated from the SEM images obtained by using an intercept method. A straight line was drawn through the photomicrographs that show the grains structure. The grains intersected by each line segment are counted, the line length is then divided by an average of the number of grains intersected, taken over all the line segments.

\subsection{Volume Fraction of Porosity in the Sintered Samples}

For the materials fabricated by the sintering process, porosity may remain inside the sintered samples and affect the thermal and mechanical properties of the materials. The existence of porosity could result in the reduction in measured thermal conductivity compared with that of fully dense materials with no porosity. Similarly, elastic modulus of the material could also be reduced due to the existence of porosity. Thus, knowledge 
of the volume fraction of porosity as well as the pore size and shape are required for the corrections of the measured thermal conductivity and elastic modulus.

The determination of the average pore size distribution and overall porosity was accomplished using the Clemex Vision PE 4.0 Image Analysis System, Clemex Technologies, Inc. The raw images were loaded into the software and an automated routine was established to ensure invariant image analysis of the images. The porosity was estimated by setting up a threshold according to its different gray level when compared with the dense part. However, the arbitrary choice of the gray level threshold may cause inaccuracies in the data. Digital area analysis determined the number of pores, their respective size fractions, and the total porosity area. If the pore was assumed to be spherical, the volume fraction of porosity was approximately equal to the area fraction.

The backscattered electron images obtained from SEM were used for the image analysis so that higher contrast could be used to increase the accuracy of the data. About 100 pores per specimen were measured to obtain the average pore size; and at least 5 representative BSE images per specimen were used to obtain the pore volume fractions.

\subsection{Elemental Composition Analysis}

The quantitative elemental composition analysis of the doped samples was conducted using an energy-dispersive x-ray spectrometer (EDS) with a $\mathrm{Si}$ (Li) semiconductor detector placed at the tip of the detector, which was installed in the Philips XL30 SFEG SEM. The detection limit in bulk materials of this EDS was about $0.1 \mathrm{wt} \%$ and resolution was $142.07 \mathrm{eV}$. However, for the light elements such as oxygen with a low-energy 
$(<0.7 \mathrm{KeV}) \mathrm{x}$-ray line, the atomic fraction or weight fraction would not be accurate due to its uncertain large mass absorption coefficient. This inaccuracy could also affect the data of other elements. Thus, only semi-quantitative EDS results could be given in this measurement.

The distributions of all the elements including the dopant cations within the doped samples were examined, respectively. The selection of the points for elemental composition analysis was performed manually using a backscattered electron image. About 8 points were selected within the area of grains and across the grain boundaries, and the beam size at each point detected was $\sim 30 \mathrm{~nm}$ in diameter. Data was collected in $0.3 \mathrm{eV}$ increments with a dwell time at $50 \mu \mathrm{sec}$ each step. The tilted angle of the sample was at $0^{\circ}$.

Specimen preparation: The specimens used for elemental analysis were polished using diamond-coated polishing films and diamond up to $0.25 \mu \mathrm{m}$; and then ultrasonically cleaned and firmly attached to the sample stage. The surfaces were carbon-coated using an E306 Edwards vacuum coater to prevent charging effects due to the non-conductivity of zirconia-based ceramics.

\subsection{Phase Identification}

In the sintered zirconia based materials, the room temperature phase might contain monoclinic, tetragonal or cubic phases. To identify those phases present at room temperature, as well as to estimate the proportions of different phases in multiphase specimens, the qualitative $\mathrm{x}$-ray diffraction (XRD) technique was used and the methods 
are described in this section.

The principle of XRD is based on Bragg's law. When a monochromatic X-ray beam with wavelength $\lambda$ is incident on the lattice planes in a crystal at an angle $\theta$, there is a path difference $\Lambda$ of x-rays diffracted by two adjacent rows of atoms in a crystal: $\Lambda=2 d \sin \theta$. Where $d$ is the spacing between the planes in the atomic lattice, as shown in Figure 52. By varying the incident angle $\theta$, constructive interference occurs when the path difference satisfies the Bragg's law conditions:

$n \lambda=2 d \sin \theta_{B} \quad n=1,2,3, \cdots$

(Eq. 56)

where, $\theta_{B}$ is called the Bragg angle.

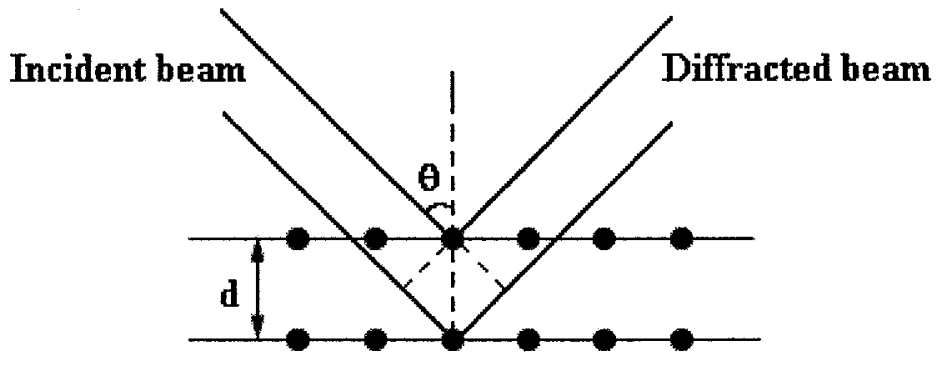

Figure 52 Schematic diagram of x-rays diffracted by two adjacent rows of atoms in a crystal.

For Bragg reflection to occur from monoclinic, tetragonal or cubic structures, the following relationships must be satisfied:

Cubic:

$\sin \theta_{B}=\frac{\lambda}{2 a} \sqrt{h^{2}+k^{2}+l^{2}}$ 
Tetragonal:

$\sin \theta_{B}=\frac{\lambda}{2} \sqrt{\frac{h^{2}+k^{2}}{a^{2}}+\frac{l^{2}}{c^{2}}}$

Monoclinic:

$\sin \theta_{B}=\frac{\lambda}{2 \sin \beta} \sqrt{\frac{h^{2}}{a^{2}}+\frac{k^{2} \sin ^{2} \beta}{b^{2}}+\frac{l^{2}}{c^{2}}-\frac{2 h l \cos \beta}{a c}}$

where $h, k$ and $l$ are the Miller's indices; $a, b$ and $c$ are the lattice parameters; and $\beta$ is the interaxial angle between $b$ and $c$.

For a certain crystal structure, the Bragg angle $\theta_{B}$ varied with Miller indices. Thus, an xray diffraction pattern with the Bragg angle and intensities of the resultant diffraction peaks could be obtained by scanning the sample over a certain $2 \theta$-range. From the position and relative intensities, it could be determined which phase of the material is present. Or the identification could be achieved by comparing the x-ray diffraction pattern of an unknown sample with an internal reference database of crystalline phases.

To determine the relative amounts of each phase (crystal structure) in a multiphase specimen, the relative integrated intensities of selected x-ray diffraction peaks were evaluated using the direct comparison method [115]. If the diffraction intensity by one type of crystal structure was described as [117]:

$I_{i}(h k l)=K_{e} \cdot K_{i}(h k l) \cdot v_{i}$

(Eq. 57)

The volume of a certain phase $v_{i}$ in a multiphase specimen can therefore be obtained from (Eq. 57) and expressed as: 
$v_{i}=I_{i}(h k l) / K_{e} \cdot K_{i}(h k l)$

(Eq. 58)

where $I_{i}(h k l)$ is the intensity of the scattered wave from the $(h k l)$ planes of phase $i ; K_{e}$ is a constant for a particular experimental system, independent of diffraction substances; $K_{i}(h k l)$ is a factor related to diffraction reflection $(h k l)$ from the crystal structure of phase $i$; and $v_{i}$ is the volume fraction of phase $i$ in a multiphase specimen.

The volume fraction of this phase can also be calculated using the following equation:

$$
\frac{v_{i}}{\sum_{i=1}^{n} v_{i}}=\frac{I_{i}(h k l) / K_{i}(h k l)}{\sum_{i=1}^{n} I_{i}(h k l) / K_{i}(h k l)}
$$

where $n$ is the number of phases present in the specimen.

The determination of volume fraction requires the values of $K_{i}(h k l)$ for each phase, which can be determined from experiment. J. R. Brandon and R. Taylor [118] have obtained the volume fraction of the monoclinic phase for materials with $m+t+c$ phase mixtures by defining the integrated intensity ratio $X_{m}$ of monoclinic phase to tetragonal/cubic phase:

$$
X_{m}=\frac{I_{m}(111)+I_{m}(11 \overline{1})}{I_{m}(111)+I_{m}(11 \overline{1})+I_{c, t}(111)}
$$

From this, the volume fraction of monoclinic phase is given as: 


$$
V_{m}=\frac{P X_{m}}{1+(P-1) X_{m}}
$$

where $P=1.3$ for the monoclinic - tetragonal/cubic system as given in Ref. [118].

Similarly, the molar fractions of the monoclinic phase and tetragonal/cubic phase in a system containing monoclinic, tetragonal, and cubic phases for each specimen was calculated using the methods presented by Miller et al. [115]. Let $M_{m}$ and $M_{c, t}$ be the molar fractions of the monoclinic phase and tetragonal/cubic phase, respectively, then the molar fraction ratio of monoclinic phase to tetragonal/cubic phase is given by [115]:

$$
\frac{M_{m}}{M_{c, t}}=0.82 \cdot \frac{I_{m}(11 \overline{1})+I_{m}(111)}{I_{c, t}(111)}
$$

where $I_{m}(11 \overline{1})$ and $I_{m}(111)$ are the integral net intensities for the monoclinic phase reflected from the $(11 \overline{1})$ and (111) planes, respectively; and $I_{c, t}(111)$ is the integral net intensity for the tetragonal or cubic phase reflected from the (111) plane.

The lattice parameters for the cubic phase and the axial c/a ratio for the tetragonal phase were determined from Bragg's law and the crystallographic relations between lattice parameters and interplanar spacing $d$. The relationships are given as below:

For the cubic phase:

$$
d_{h k l}=\left[\left(h^{2}+k^{2}+l^{2}\right) / a^{2}\right]^{-\frac{1}{2}}
$$


For the tetragonal phase:

$$
d_{h k l}=\left[\left(h^{2}+k^{2}\right) / a^{2}+l^{2} / c^{2}\right]^{-\frac{1}{2}}
$$

where $h, k$ and $l$ are the Miller indices.

From the measured lattice parameters, the yttria content in the tetragonal phase was determined using the relationship between the $c / a$ ratio and $m o l \% \mathrm{YO}_{1.5}$ by $\mathrm{U}$. Schultz [64], which is given by:

$$
m o l \% Y O_{1.5}=\frac{1.02223-c / a}{0.001309}
$$

The yttria content in the cubic phase was determined using the Aleksandrov model [26], which is given by:

$$
m o l \% Y O_{1.5}=\frac{200 \cdot(510.4-100 a)}{a-5.5197} /\left(100+\frac{510.4-100 a}{a-5.5197}\right)
$$

For 7YSZ co-doped with metal oxide, (Eq. 65) and (Eq. 66) will be used to calculate the equivalent yttria content.

The Rigaku ${ }^{\mathrm{TM}}$ XRD equipment with a $\mathrm{Cu} \mathrm{K}_{\alpha}$ radiation source $(\lambda=0.1542 \mathrm{~nm})$ was used. For each specimen, a scan was taken in the range $2 \theta=20^{\circ}$ to $100^{\circ}$ at a scan rate of $0.8 \%$ min. The $\mathrm{x}$-ray peak identification, including interplanar spacing $\mathrm{d}$, intensity, $2 \theta$ at peak and full-width at half-maximum, were performed using the $\operatorname{Jade}^{\mathrm{TM}} 3.0$ software package. 
Sample requirement: A sample size of $25 \mathrm{~mm}$ in diameter was used to allow the specimen to be held securely in front of the x-ray beam. To avoid contamination, the samples were ultrasonically cleaned and blow-dried before the XRD measurements.

\subsection{Phenomena of Phase Transition}

To observe the phase transition and measure the specific heats of the sintered materials, thermal analysis was performed using heat flux differential scanning calorimetry (DSC), which involves the detection of changes in the thermal energy (enthalpy) or the specific heat of a sample with temperature.

\subsubsection{Basic Principle and Method of DSC}

In heat flux DSC, the test sample and an inert reference material are enclosed in the same furnace and maintained at an identical temperature. Upon heating or cooling, the rate of heat flow to the sample and to the reference is different due to their special specific heats. The relationship of differential heat flow versus temperature is therefore recorded as a DSC thermogram. Any transition associated with absorption or evolution of heat causes a discontinuity in the heat flow and results in a peak on the curve. The area under the peak is proportional to the total enthalpy change. Thus, transition temperature peak and the enthalpy produced can be determined from DSC thermograms.

A Netzsch DSC 404C Pegasus calorimeter (Netzsch-Geratebau GmbH, Germany) was used to conduct the experiment in this study. Figure 53 is the schematic illustration of the DSC set up. The test specimen $\mathrm{S}$ and the inert reference material $\mathrm{R}$ are placed in 
crucibles in a furnace on two constantan platforms, which are on a constantan disc. Chromel-constantan thermocouples are located on the underside of each platform to measure the temperatures. Masses of each specimen and both crucibles are recorded before each DSC run. Heating and cooling are controlled through a TASC 414/4 controller (Netzsch-Geratebau GmbH, Germany) linked to PC based software. In each DSC run, two temperature cycles were selected. In the first cycle, temperature was increased from $100^{\circ} \mathrm{C}$ to $1400^{\circ} \mathrm{C}$ at a heat rate of $20^{\circ} \mathrm{C} / \mathrm{min}$. Samples were held at $1400^{\circ} \mathrm{C}$ for 5 minutes, the temperature was then decreased from $1400^{\circ} \mathrm{C}$ to $500^{\circ} \mathrm{C}$ at a cooling rate of $20^{\circ} \mathrm{C} / \mathrm{min}$. In the second cycle, again the temperature was increased to $1400 \mathrm{C}$ at $20^{\circ} \mathrm{C} / \mathrm{min}$ and after 5 minutes holding, the samples were cooled to $500^{\circ} \mathrm{C}$ at a slow cooling rate of $5^{\circ} \mathrm{C} / \mathrm{min}$. The temperature program is shown in Figure 54 while the DSC equipment is shown in Figure 55. During the DSC running, an inert argon gas was used to protect the DSC cell. However, in such an environment, some oxygen reduction occurred in the experiment, which prevented the data from the second DSC run from exactly replicating the first.

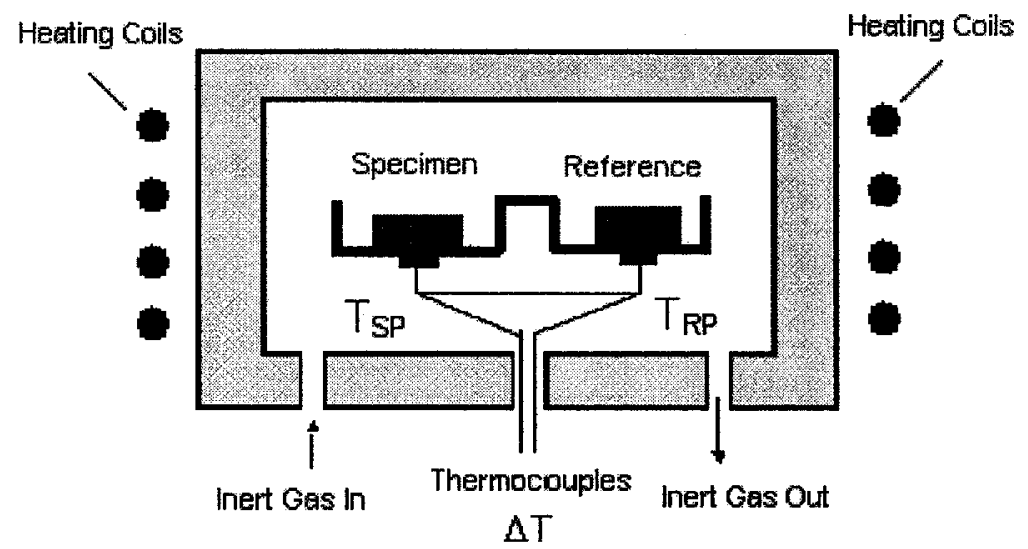

Figure 53 Schematic illustration of the heat flux DSC cell. 


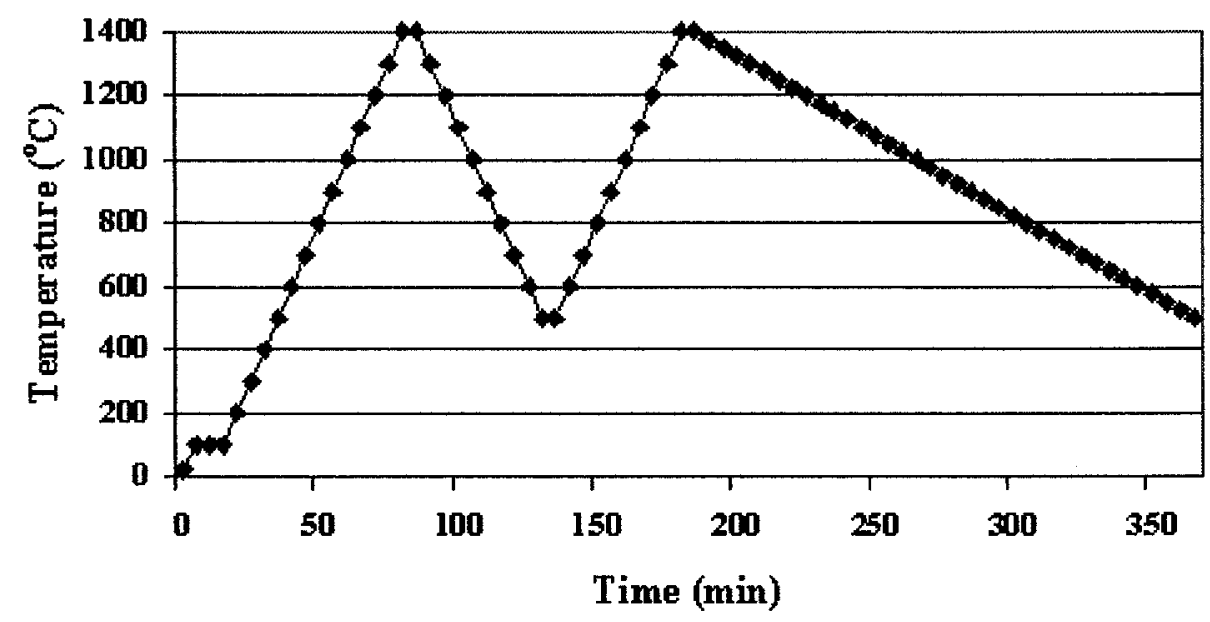

Figure 54 DSC temperature program with two heating cycles.

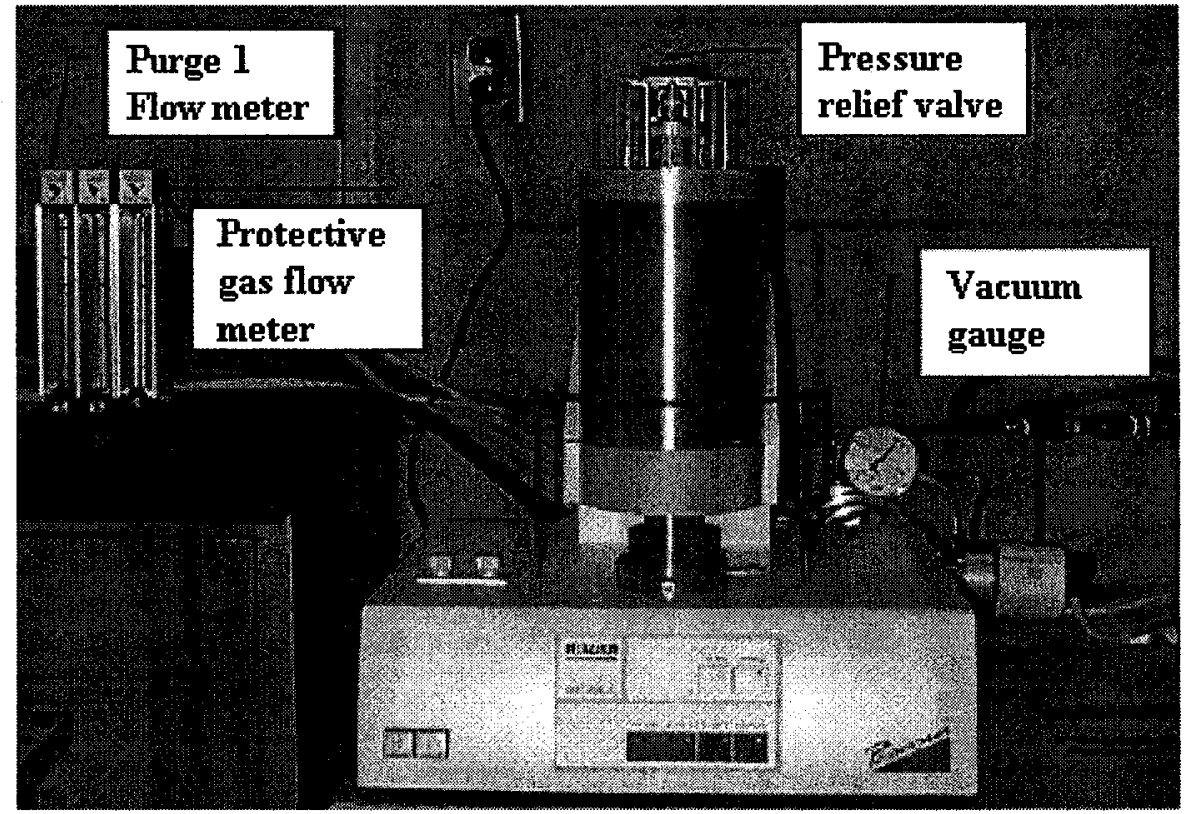

Figure 55 DSC equipment: Furnace /Specimen holder part [119].

\subsubsection{Specific Heat Measurement}

The principle for measuring specific heat of each sample using DSC was based on the 
following equation:

$C_{P \cdot s}=\frac{m_{s \tan }}{m_{s}} \cdot \frac{D S C_{s}-D S C_{B a s}}{D S C_{s \tan }-D S C_{B a s}} \cdot C_{P \cdot s \tan }$

(Eq. 67)

where $C_{P \cdot s}$ is the specific heat of the sample; and $C_{P \cdot s \text { tan }}$ is the known standard specific heat of sapphire; $m_{s}$ and $m_{s \tan }$ are the masses of the sample and sapphire, respectively; and $D S C_{s}, D S C_{s \tan }$, and $D S C_{B a s}$ are the DSC curves of the sample, sapphire, and baseline respectively.

Thus, to calculate the specific heat of the specimen, three measurements were required: a baseline measurement was performed by running DSC for two empty crucibles; a standard measurement was done by using a sapphire disc as a standard sample; and a sample measurement was carried out as described earlier. Within this series of measurements, the following test parameters must be identical: atmosphere in the measuring cell; argon flow rate for operation; initial temperature; heat rate and scanning rate; mass of crucible and lid; and the position of crucible in the cell. Most importantly, when selecting the table of standard values, one must be sure that the calibration measurement and the table are based on the same calibration material. Then the measurements of "sample + correction" and "standard + correction" were loaded into the Proteus analysis software, which is a software used for analyzing the DSC results. By selecting the heating segment with a heat rate of $20^{\circ} \mathrm{C} / \mathrm{min}$ and the $\mathrm{Cp}$-standard table for sapphire, the specific heat of each sample was calculated by using this software. 
Sample preparation: The weight of the samples for DSC was between $35-45 \mathrm{mg}$, the dimension of the samples was less than that of the crucible so that the samples could be contained within the crucible. All the samples were ultrasonically cleaned and blowdried.

\subsection{Mechanical Properties of the Sintered Samples}

The elastic moduli of doped materials and the baseline 7YSZ were determined using a CSM Nano-indentation Tester, a high precision instrument for measuring mechanical properties from small volumes of material.

The testing principle is based on the Depth-Sensing Indentation technique: a load is increasingly applied on an indenter tip, which is driven into the surface of a material to be tested. Upon reaching a maximum value, the load is gradually reduced until a complete unloading is achieved. A curve of the indentation load versus the corresponding penetration depth of the indenter is then generated for each loading/unloading cycle. A schematic of the indentation geometry at maximum load for an ideal conical indenter and the indentation load-displacement curve are shown in Figure 56 [120], where $h_{\max }$ is the maximum indentation depth under the maximum load $F_{\max } ; h_{c}$ is the contact depth; and $h_{p}$ is the residual plastic deformation.

Based on the assumption that unloading is fully elastic, a reduced elastic modulus is evaluated from the unloading curve [120], using the expression:

$$
E=\frac{\pi^{1 / 2}}{2} \cdot \frac{S}{a^{1 / 2}}
$$


(Eq. 68)

where $S$ is the stiffness at the maximum loading point and can be determined from the unloading data; $a$ is the contact area corresponding to the arbitrary maximum indentation load and is evaluated from the contact depth $h_{c}$ and the known geometry of the indenter. The contact depth $h_{c}$ cannot be measured directly. It is commonly estimated using the Oliver \& Pharr method [120], which is expressed by:

$$
h_{c}=h_{p}-\theta \cdot \frac{F_{\max }}{S}
$$

where $h_{p}$ and $F_{\max }$ were defined in Figure $56 ; \theta$ is a parameter associated with the geometry of indenter and is 0.72 .0 .75 and 1.0 for cone, spherical, and flat-ended cylindrical punch, respectively.
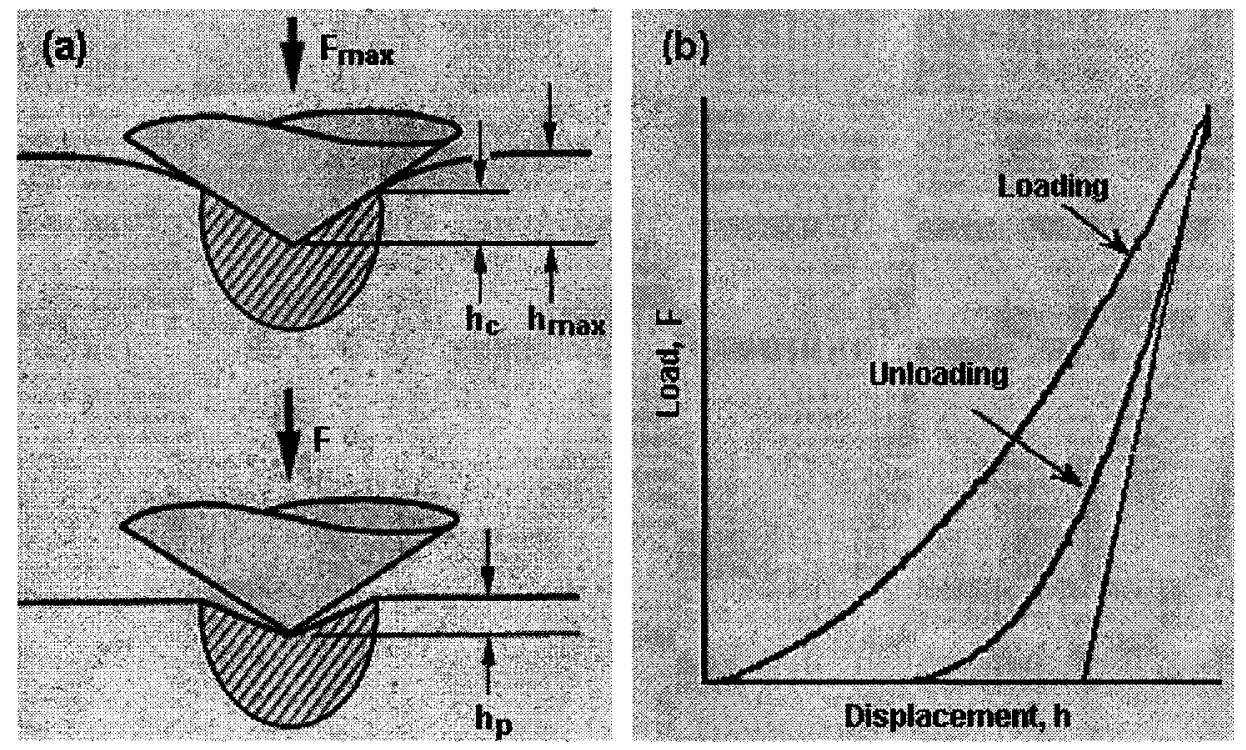

Figure 56 (a) Schematic of the indentation testing for an ideal conical indenter and (b) the indentation load-displacement curve [120]. 
There is a relationship between the reduced elastic modulus $\mathrm{E}$ and the elastic modulii $E_{i}$ and $E_{s}$ of indenter and specimen,

$$
\frac{1}{E}=\frac{\left(1-v_{i}^{2}\right)}{E_{i}}+\frac{\left(1-v_{s}^{2}\right)}{E_{s}}
$$

where $v_{\mathrm{i}}$ and $v_{\mathrm{s}}$ are the Poisson ratios of the indenter and specimen, respectively. With the known parameters of the indenter, the elastic modulus of the tested sample is determined. In the testing, a force of $50 \mathrm{mN}$ was applied to each sample, and at four points of each sample surface was measured to ensure accuracy of the measurements. Since the measured elastic modulus is affected by the porosity existing inside each sintered sample, the zero-porosity elastic modulus is calculated using Mackenzie's equation [121]:

$E=E_{0} \cdot\left(1-1.9 P+0.9 P^{2}\right)$

(Eq. 71)

where $P$ is the volume fraction of porosity, which is obtained from image analysis, and $E_{0}$ is the elastic modulus of a nonporous material.

Sample preparation: The sample discs were prepared with dimensions of $10 \mathrm{~mm}$ in diameter and $2 \mathrm{~mm}$ in thickness. The planar surfaces of the samples were parallel to the testing surface; all the samples surface were polished using diamond abrasive papers down to $6 \mu \mathrm{m}$ to reduce the surface roughness. Before testing, the samples were ultrasonically cleaned and blow-dried. 


\subsection{Measurement of Thermal Diffusivities}

Thermal conductivities of the doped materials were evaluated from the relationship: $\lambda=\alpha \cdot C_{P} \cdot \rho$

where $\rho$ was the bulk density $\left(\mathrm{kg} / \mathrm{m}^{3}\right), C p$ was the specific heat capacity $(\mathrm{J} / \mathrm{kg} \cdot \mathrm{K})$, and $\alpha$ was the thermal diffusivity $\left(\mathrm{m}^{2} / \mathrm{s}\right)$. To obtain the values of thermal conductivities of the doped materials, the thermal diffusivity of the doped bulk materials were required.

The thermal diffusivities of the zirconia-based materials were measured using a laser flash technique at IMI, NRC in Montreal. The basic principle of the laser flash measurement involved rapid heating of one side of the specimen using laser energy and measuring the temperature rise on the opposite side. Then the time required for the heat to travel through the sample and cause the temperature to rise on the rear face could be used to measure the diffusivity.

A schematic of the diffusivity measurement using the laser flash technique is shown in Figure 57, where a laser beam from a single pulse Nd:YAG laser with $70 \mu$ s duration, $1.0 \mathrm{~J}$ energy and $1.064 \mu \mathrm{m}$ wavelength was projected over the full face of the sample, which was placed inside an air furnace. The heat flux absorbed from the pulse laser was transported to the other side of the material. An InSb infrared detector was used to monitor the temperature history at the rear surface of the specimen over the range 20 to $800^{\circ} \mathrm{C}$. From the temperature rise versus time curve recorded, the time $t_{1 / 2}$ and $t_{5 / 6}$, which are the time periods corresponding to a temperature rise to half and five-sixths of the 
maximum temperature at the rear surface of the sample, respectively, were obtained. Then the thermal diffusivity was calculated using the relationships given by [122]:

$t_{5 / 6}^{*}=\alpha \cdot t_{5 / 6} / e^{2}$

$t_{5 / 6}^{*}=0.968-1.6382\left(t_{1 / 2} / t_{5 / 6}\right)+0.6148\left(t_{1 / 2} / t_{5 / 6}\right)^{2}$

(Eq. 74)

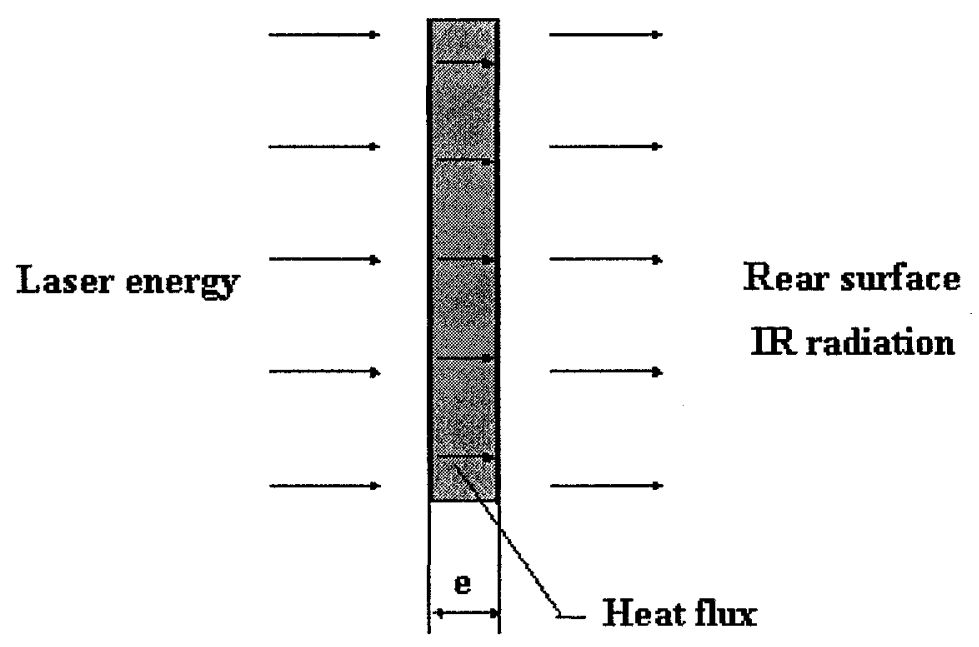

Figure 57 Schematic of laser flash diffusivity measurement.

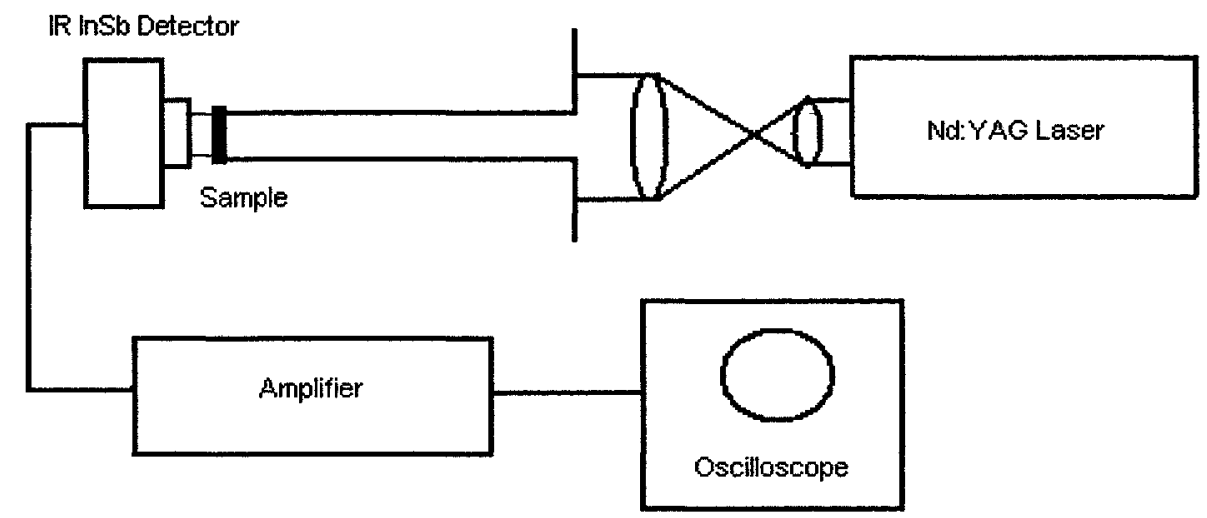

Figure 58 Setup for thermal diffusivity measurement. 


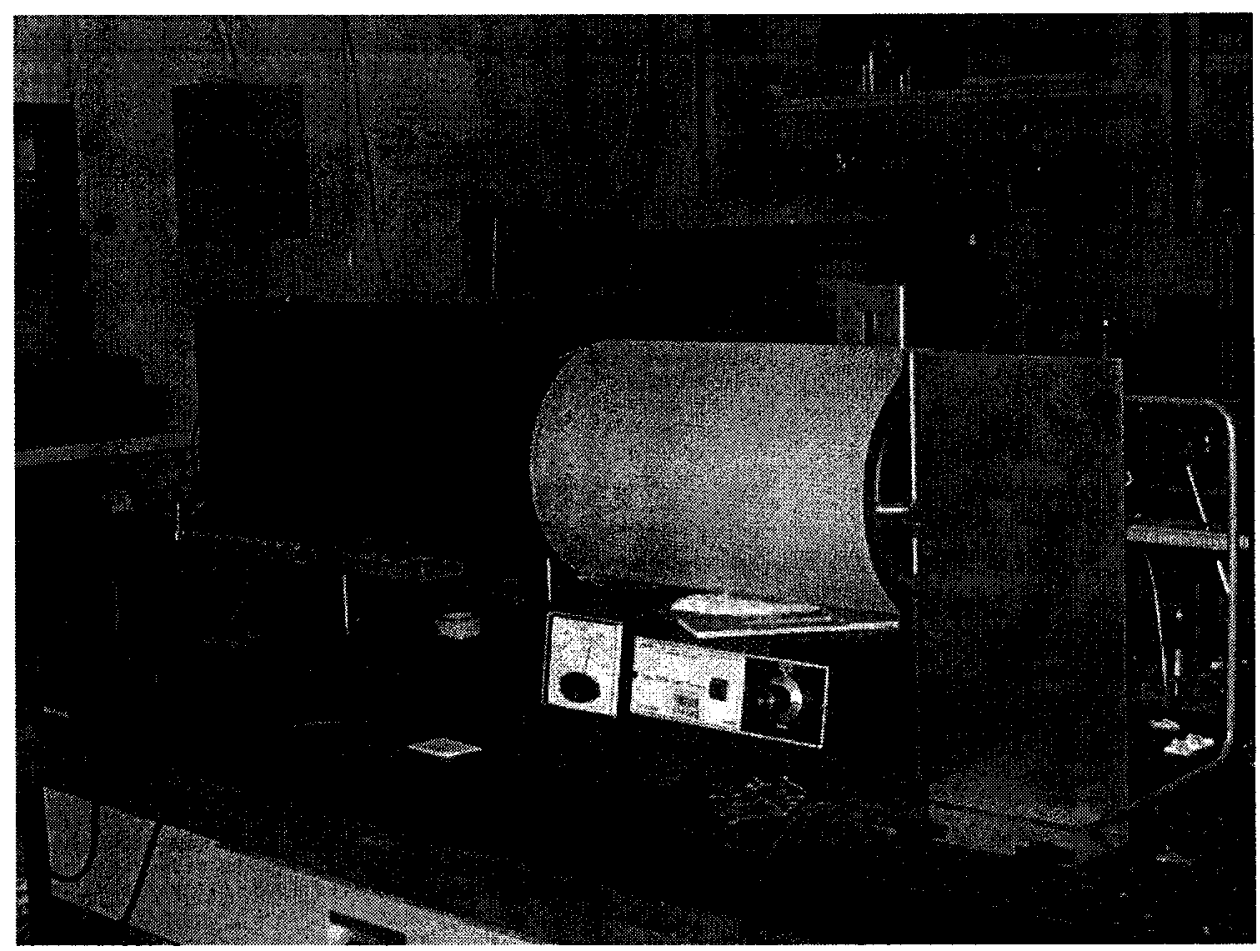

Figure 59 Equipment for measuring thermal diffusivity

The test setup and the equipment used are shown in Figure 58 and Figure 59. To obtain a reliable diffusivity result, each measurement was repeated 3 times, and the reported values were the average of 3 measurements.

The samples had a nominal diameter of $10 \mathrm{~mm}$ and the thicknesses were between $400 \sim 700 \mu \mathrm{m}$. The surfaces at both sides were ground with diamond abrasive papers down to $6 \mu \mathrm{m}$. To eliminate the effect of radiation emitted from inside the zirconia-based materials and penetration of the laser beam through the sample due to the semitransparency of the ceramic materials, prior to the measurement, the samples were sputter-coated with $0.15 \mu \mathrm{m}$ thick film of gold-palladium so that the samples were opaque. 


\section{Results and Discussion}

The characteristics of the ground, alloyed powders have been examined using SEM and the results are shown in the following sections. The variations of the microstructures, the thermal, physical and mechanical properties of the sintered materials with metal oxide dopants have been examined and will be demonstrated and discussed in this chapter.

\subsection{Powder Characterization}

Powder materials in both as-received condition and after blending and 50hrs milling were examined using SEM and the results are given in the following section.

\subsubsection{SEM Images of All As-received Powders}

Figure 60 and Figure 61 show the particle morphologies of the as-received powders examined using scanning electron microscope. The original 7YSZ powder consists of highly spherical particles, which have a wide range of diameters. Each spherical powder particle appears to consist of an agglomeration of smaller crystals, as shown in Figure 60. The powders of tantalum oxide, niobium oxide and chromium oxide are very fine with loose bonding. The particle sizes of the powders are within the nanoscale range, as shown in Figure 61 (a) $\sim(c)$. The ceria, scandia and ytterbia powders constitute irregular shaped agglomerates. The particle size distribution of these agglomerates is wide, ranging from $1 \sim 15 \mu \mathrm{m}$, as shown in Figure 61 (d) $\sim(\mathrm{f})$. 


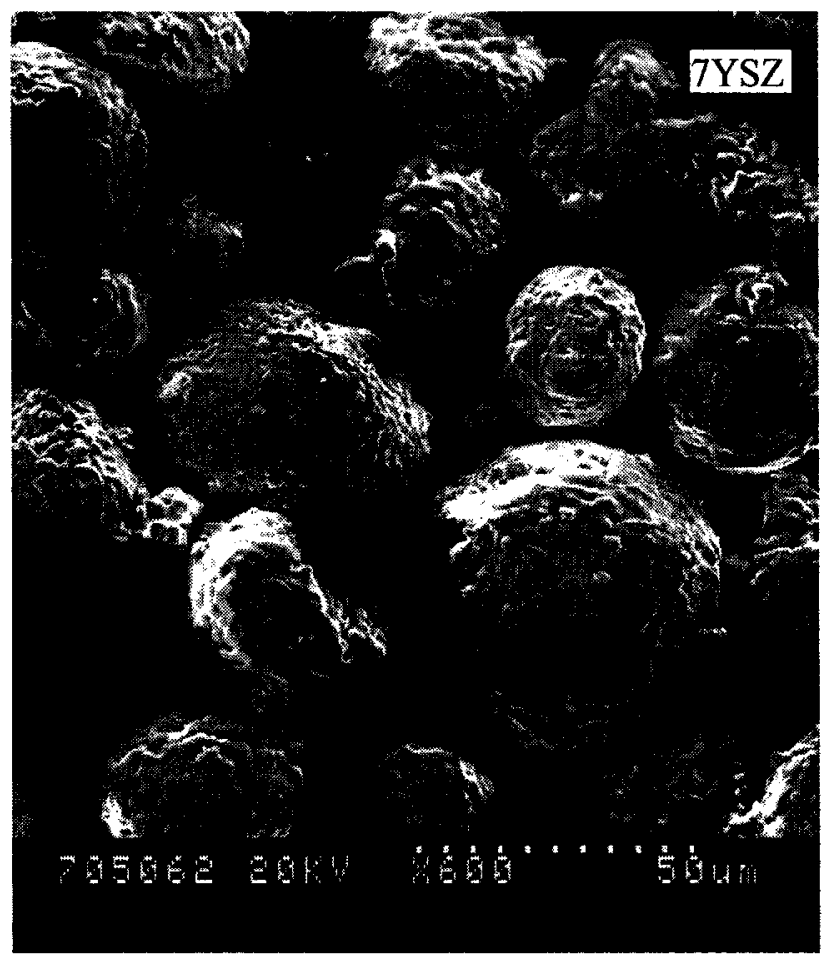

Figure 60 Morphologies of the as-received 7YSZ powders.

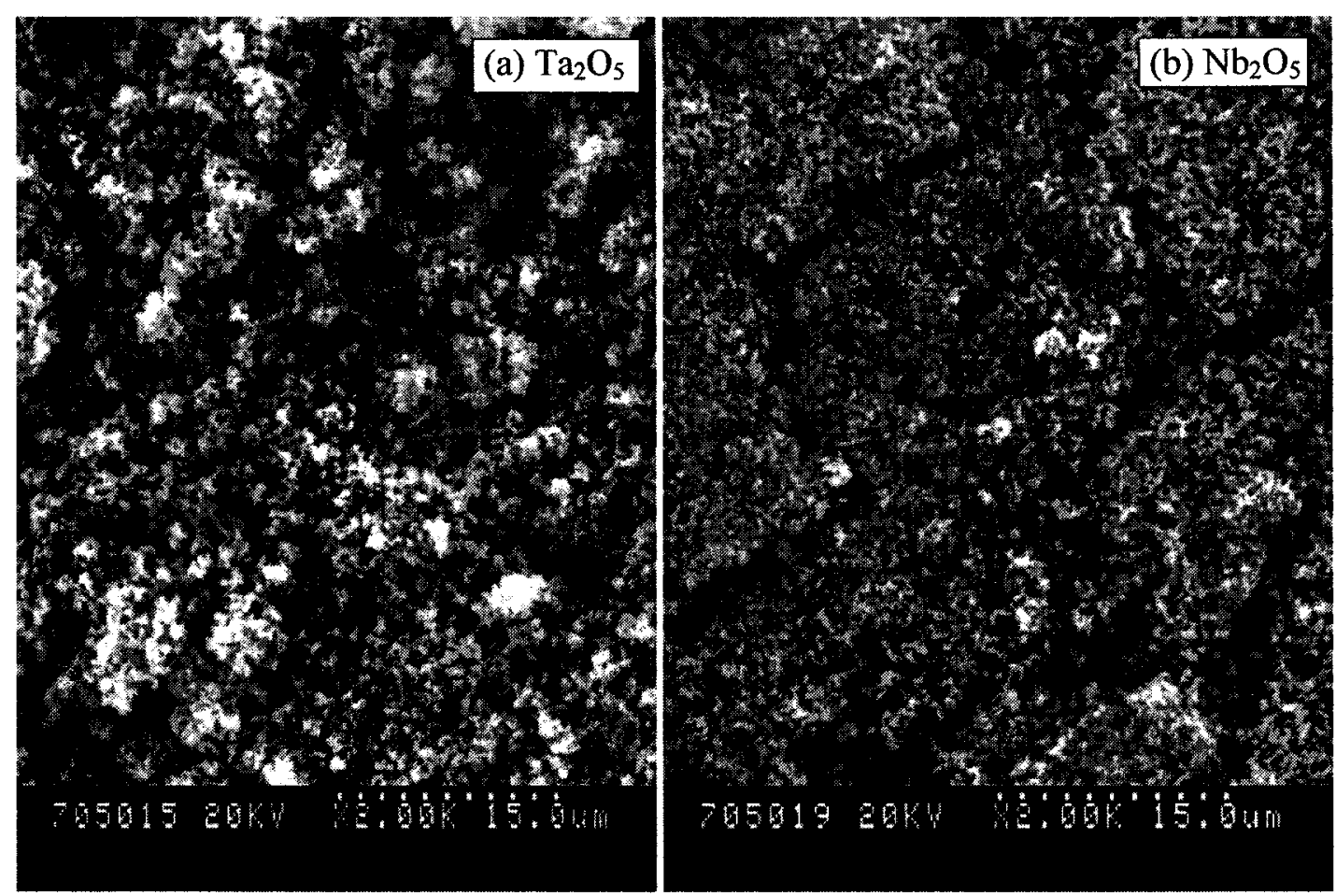



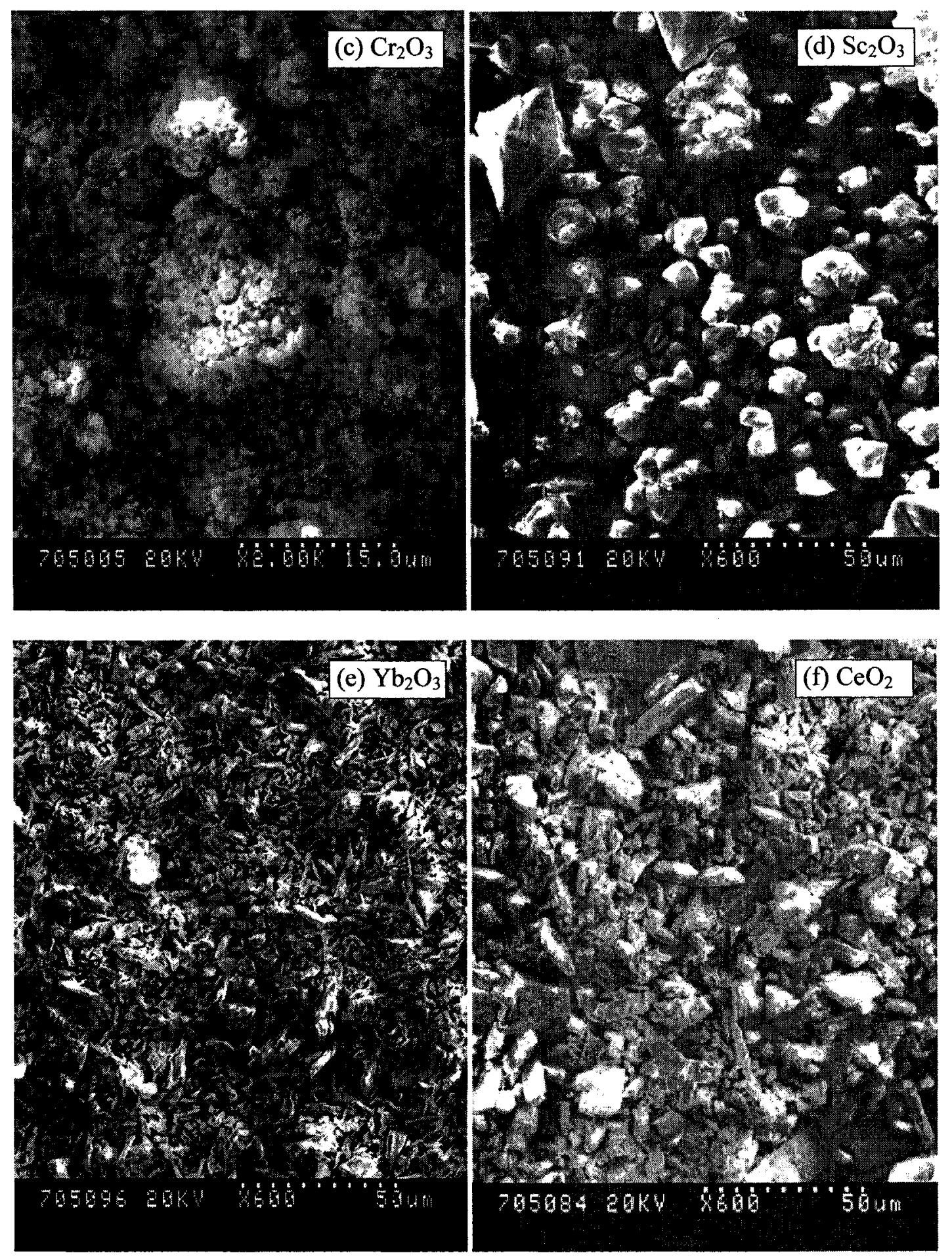

Figure 61 Morphologies of the as-received (a) $\mathrm{Ta}_{2} \mathrm{O}_{5}$ powders; (b) $\mathrm{Nb}_{2} \mathrm{O}_{5}$ powders; (c) $\mathrm{Cr}_{2} \mathrm{O}_{3}$ powders; (d) $\mathrm{Sc}_{2} \mathrm{O}_{3}$ powders; (e) $\mathrm{Yb}_{2} \mathrm{O}_{3}$ powders; (f) $\mathrm{CeO}_{2}$ powders. 


\subsubsection{SEM Images of Selected Powders after Mechanical Alloying}

The particle morphologies of 7YSZ after 50hrs ball milling are shown in Figure 62 . Compared with the as-received 7 YSZ power shown in Figure 60 , it can be found that after $50 \mathrm{hrs}$ grinding, the spherical particles of $7 \mathrm{YSZ}$ are broken into pieces and the particle sizes become smaller. Most of the particles have a size ranging from 1 to $10 \mu \mathrm{m}$ and only a small number of particles have sizes about $25 \mu \mathrm{m}$.

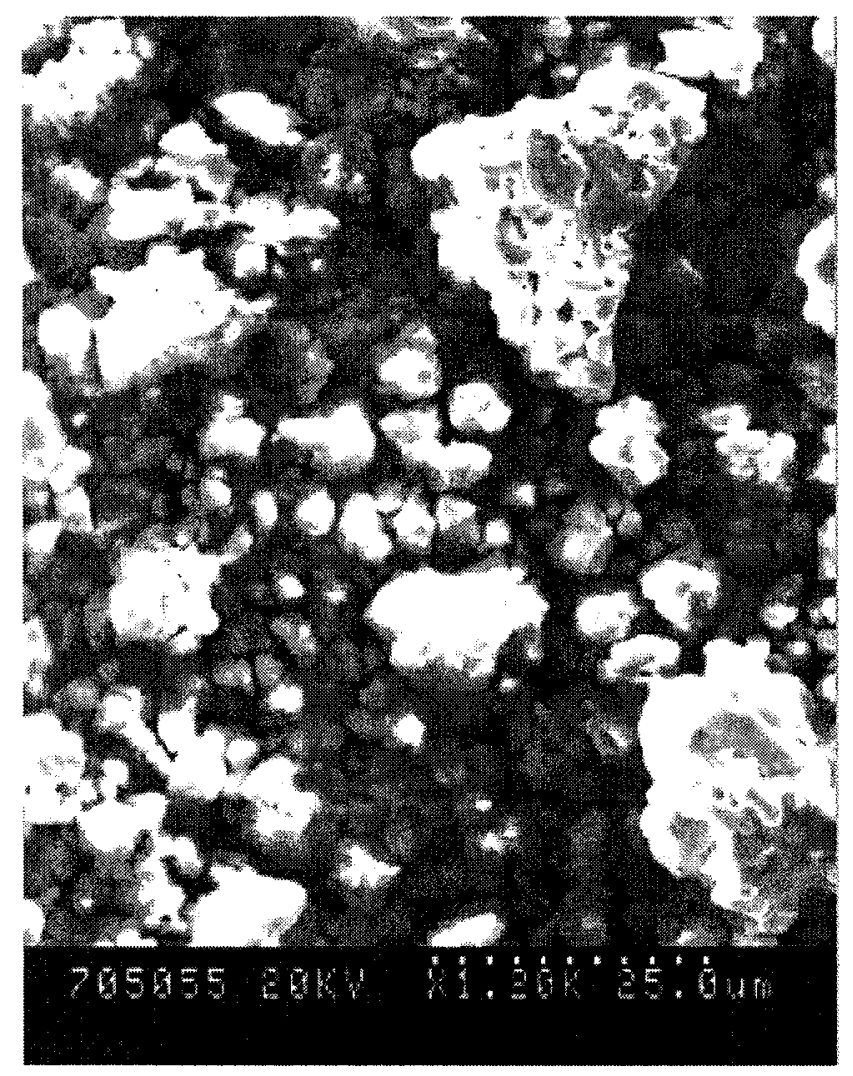

Figure 62 Particle morphologies of 7YSZ powders after 50hrs grinding.

Figure 63 shows the particle morphologies of the alloyed powers, including 3.9TaYSZ, 3.9NbYSZ, 5CrYSZ, 5ScYSZ, 5YbYSZ, and 5CeYSZ, after 50hrs blending and grinding. 

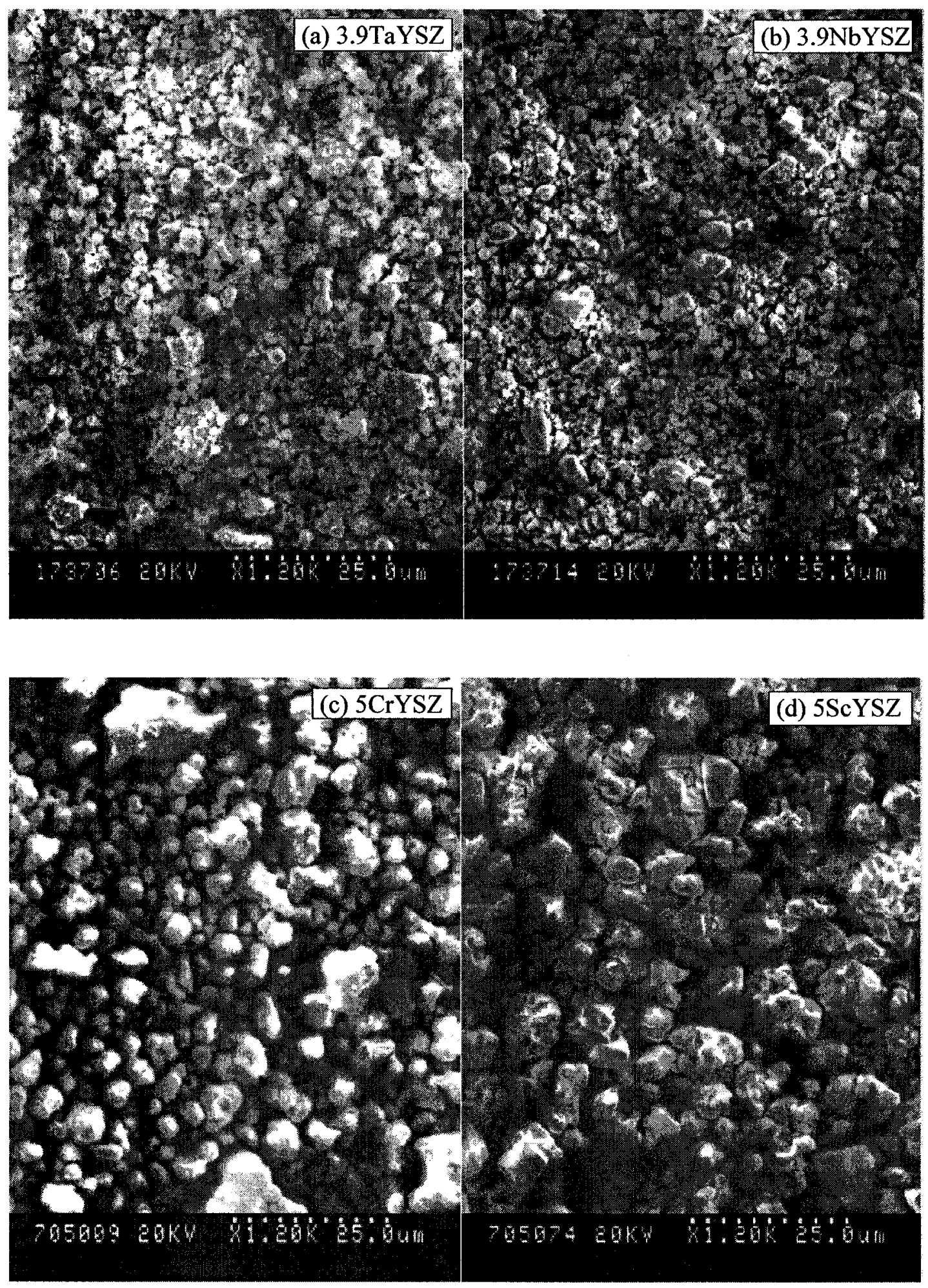


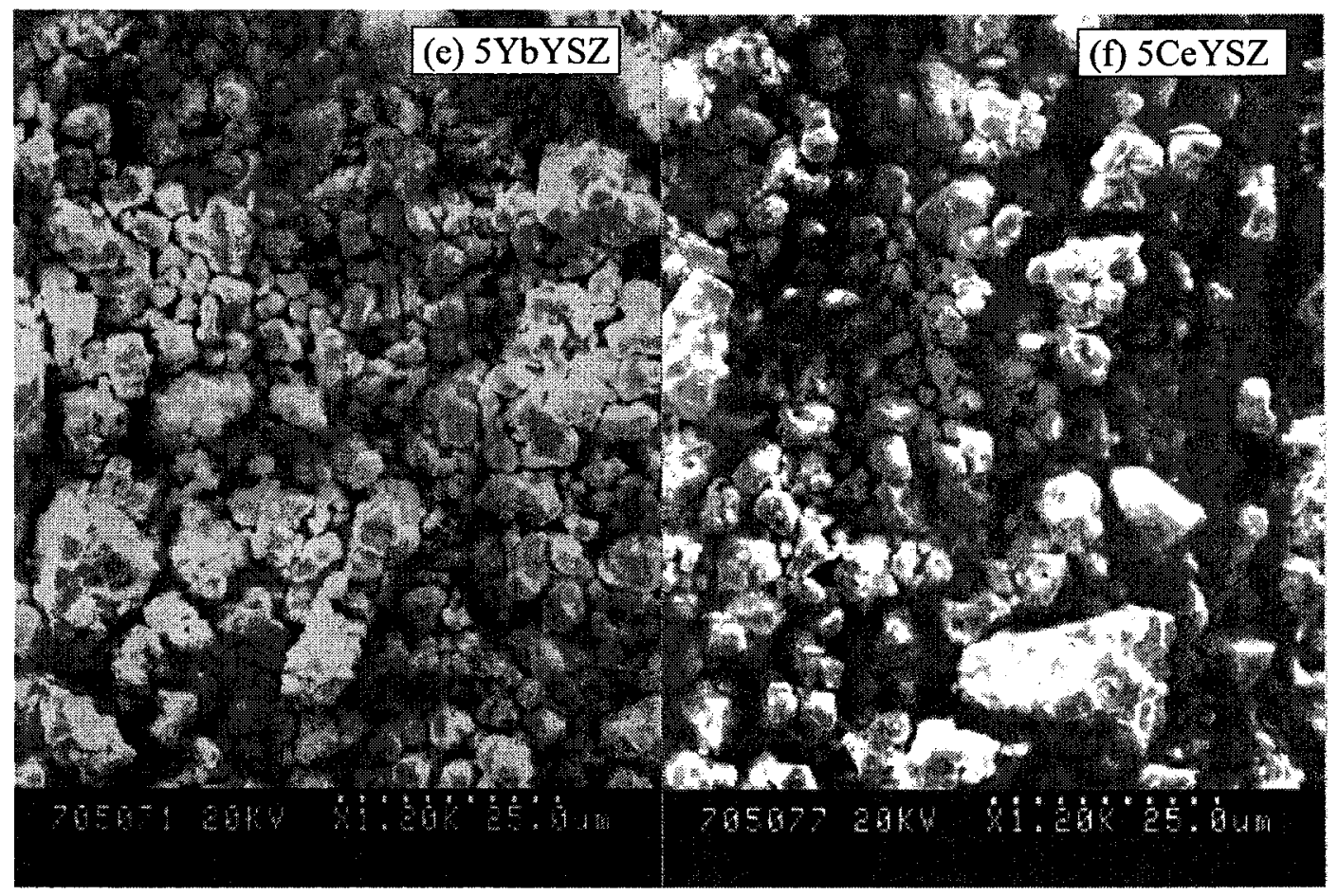

Figure 63 Morphologies of the alloyed powders after 50hrs grinding. (a) 3.9TaYSZ powder; (b) $3.9 \mathrm{NbYSZ}$ powder; (c) 5CrYSZ powder; (d) 5ScYSZ powder; (e) 5YbYSZ powder; and (f) $5 \mathrm{CeYSZ}$ powder.

The distribution of particle sizes for the alloyed 3.9TaYSZ, 3.9NbYSZ, 5CrYSZ powders are wider than those of 5ScYSZ, 5YbYSZ, and 5CeYSZ, within the range of $0.1 \sim 10$ $\mu \mathrm{m}$, whereas $5 \mathrm{ScYSZ}, 5 \mathrm{YbYSZ}$, and $5 \mathrm{CeYSZ}$ powders have narrow particle size distributions. This is because the particle sizes of the $\mathrm{Ta}_{2} \mathrm{O}_{5}, \mathrm{Nb}_{2} \mathrm{O}_{5}$ and $\mathrm{Cr}_{2} \mathrm{O}_{3}$ dopants are very small and the dopants are therefore attached to the large 7YSZ particles. The particle sizes for 5ScYSZ and 5CeYSZ powders fall within the range of $1 \sim 10 \mu \mathrm{m}$, and the $5 \mathrm{YbYSZ}$ powder is within the range of $1 \sim 15 \mu \mathrm{m}$. However, a small number of large particles were not broken completely and need further processing with increased milling times. 


\subsection{Microstructures of the Sintered Samples}

The microstructures of each sintered material including 3.9TaYSZ, 3.9NbYSZ, 5CrYSZ, 5ScYSZ, 5YbYSZ, and 5CeYSZ were examined using SEM for both sintered surfaces and fracture surfaces. For comparison, the microstructures of 7YSZ for both sintered surfaces and fracture surfaces were also examined, as shown in Figure 65 and Figure 64 (a) (f). The microstructures of the fracture surfaces (left images) were used to determine the distribution of porosity within the samples and to examine the fracture paths; and the microstructures of the sintered surfaces (right images) were used to roughly determine the average grain size using the lineal intercept method.
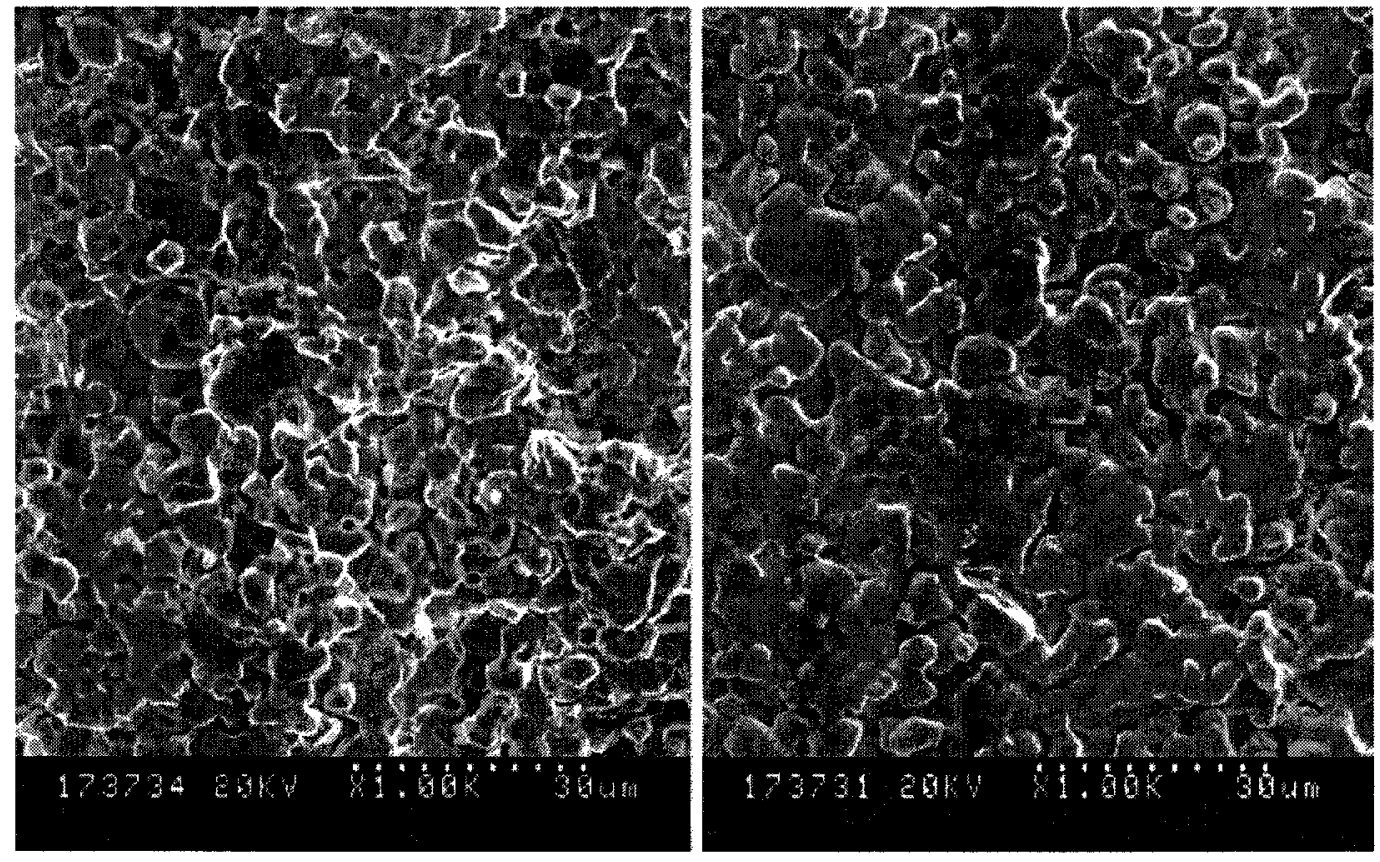

Figure 64 SEM micrographs of sintered 7YSZ. Left image: fracture section; right image: sintered surface. 


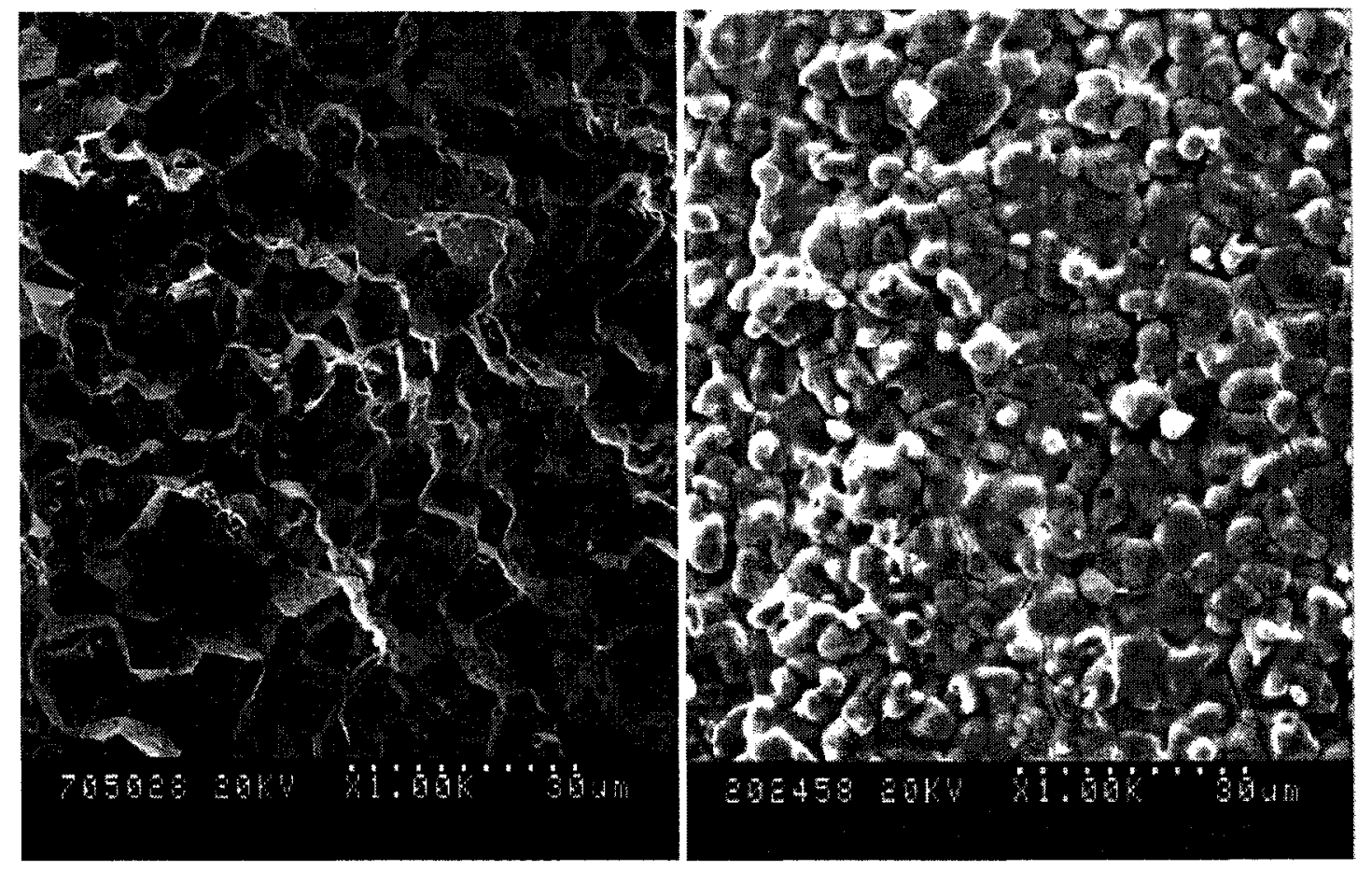

(a) 3.9TaYSZ.
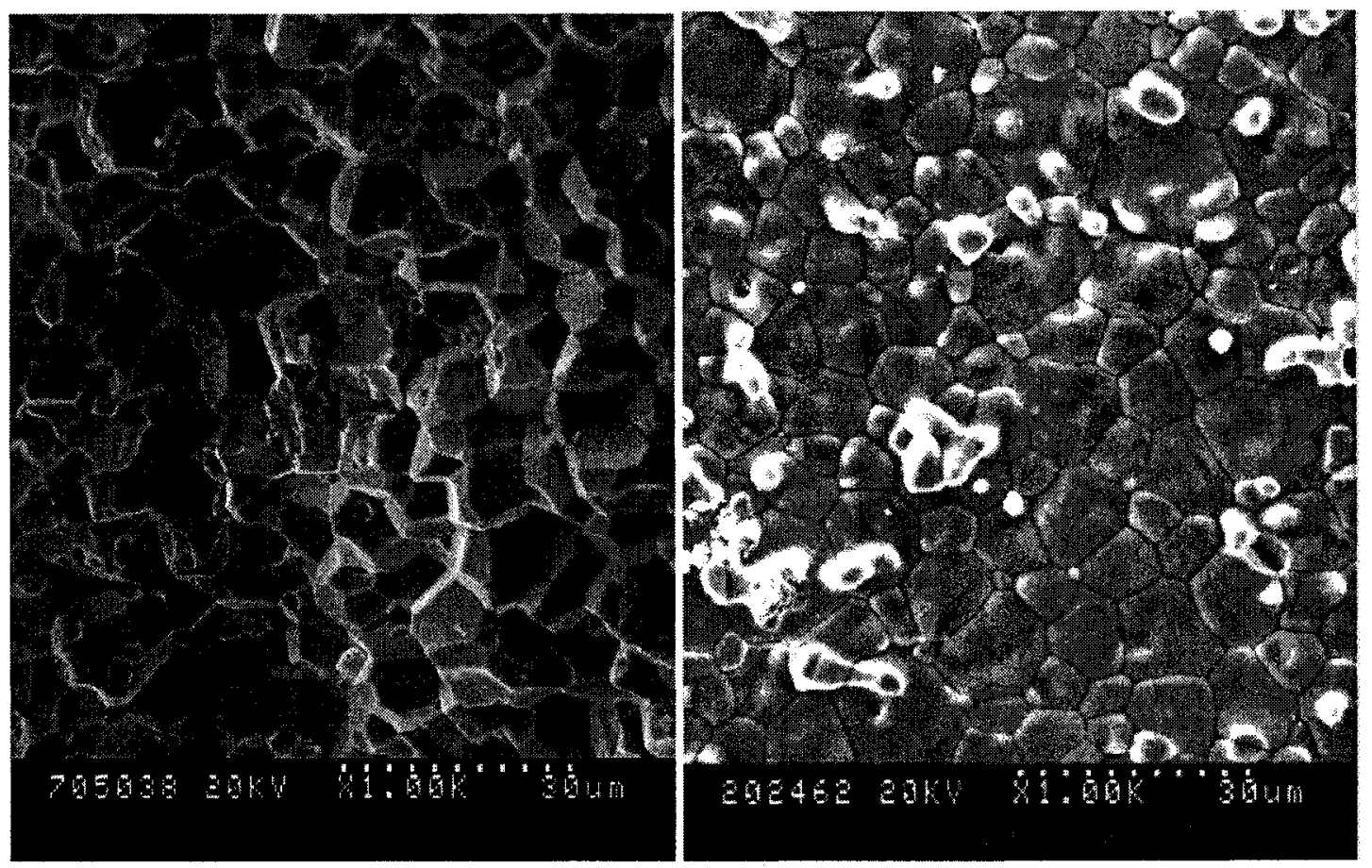

(b) $3.9 \mathrm{NbYSZ}$. 


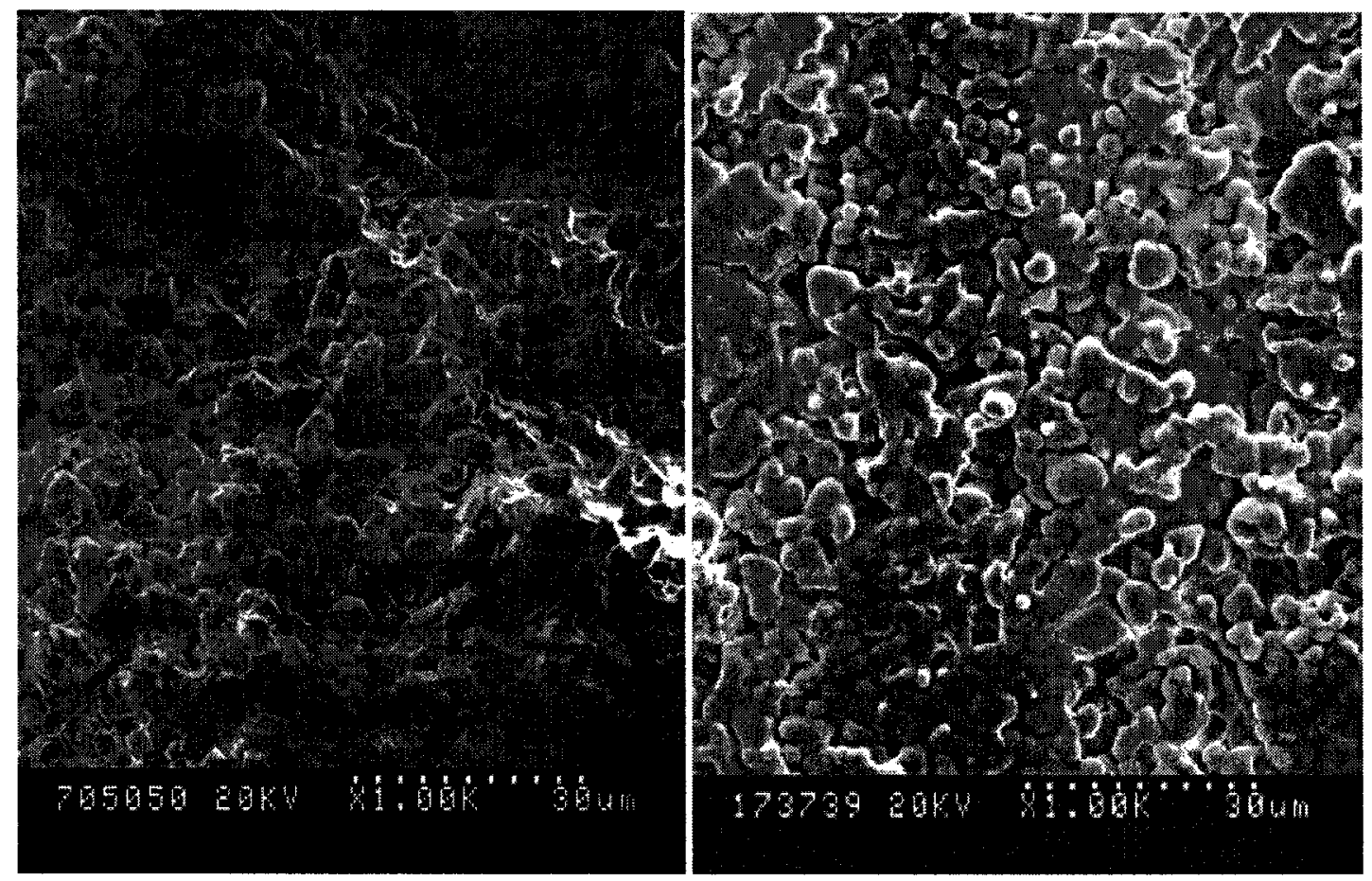

(c) 5 CrYSZ.
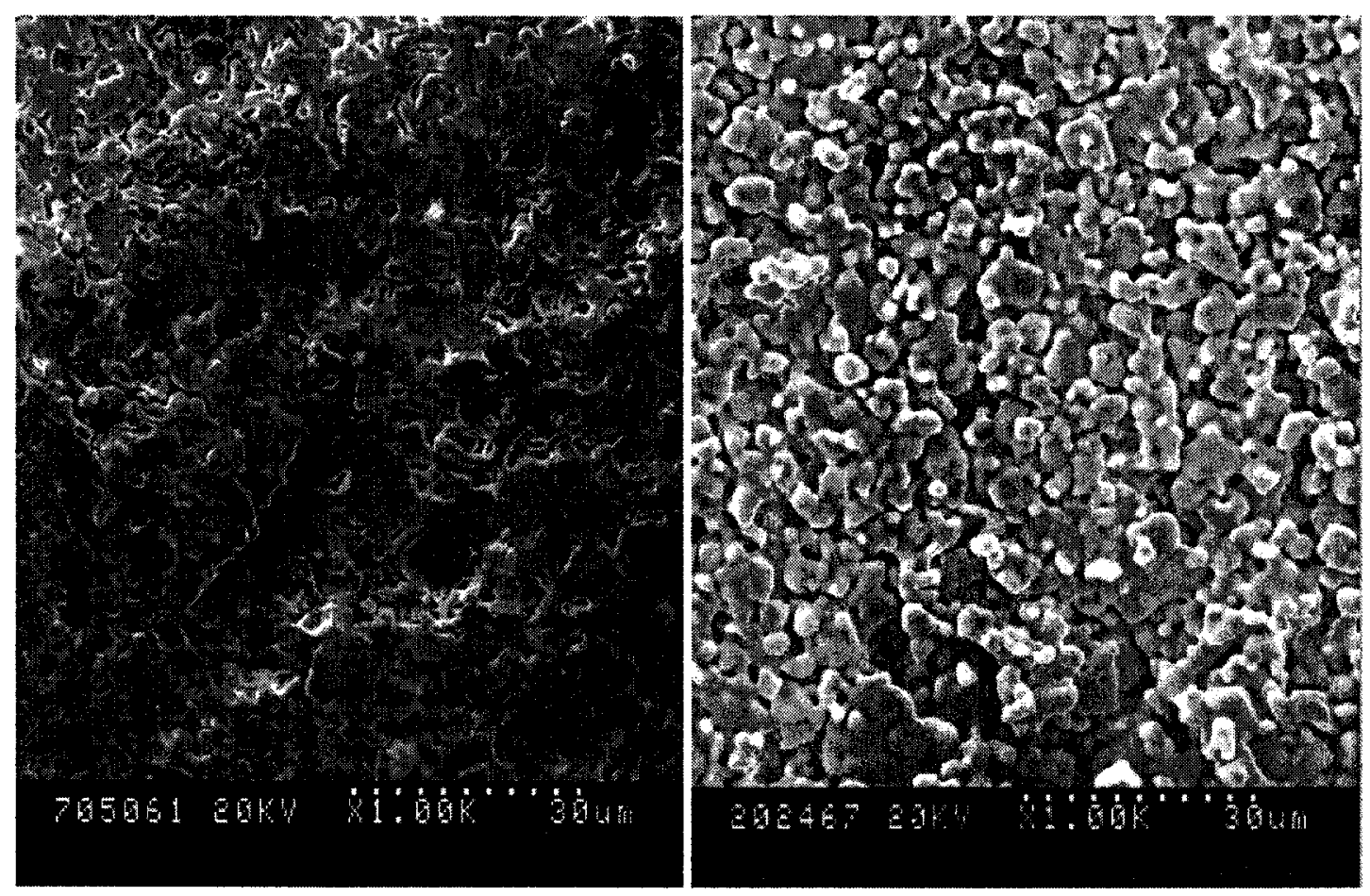

(d) 5ScYSZ. 


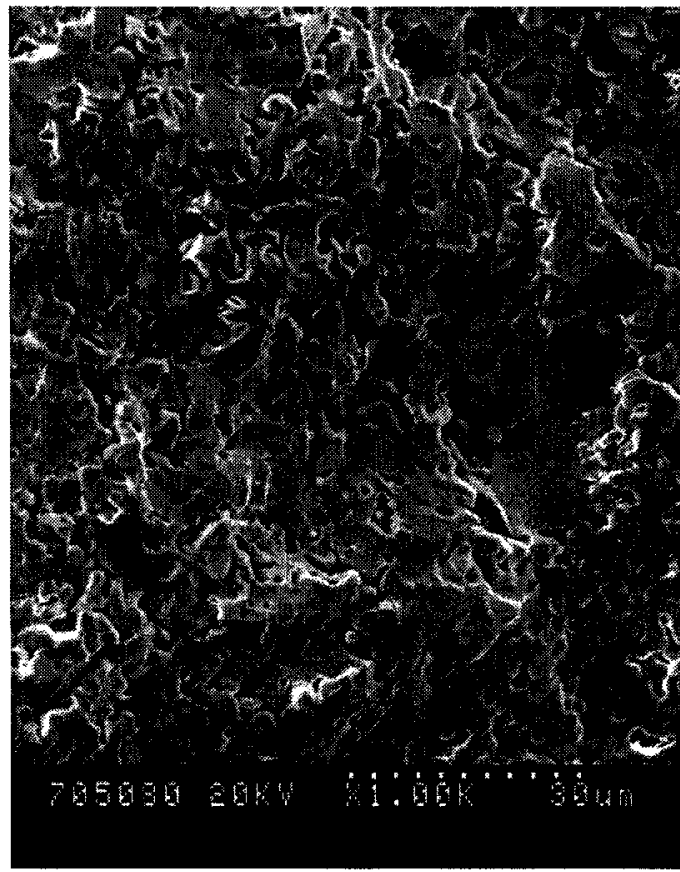

(e) 5 YbYSZ.
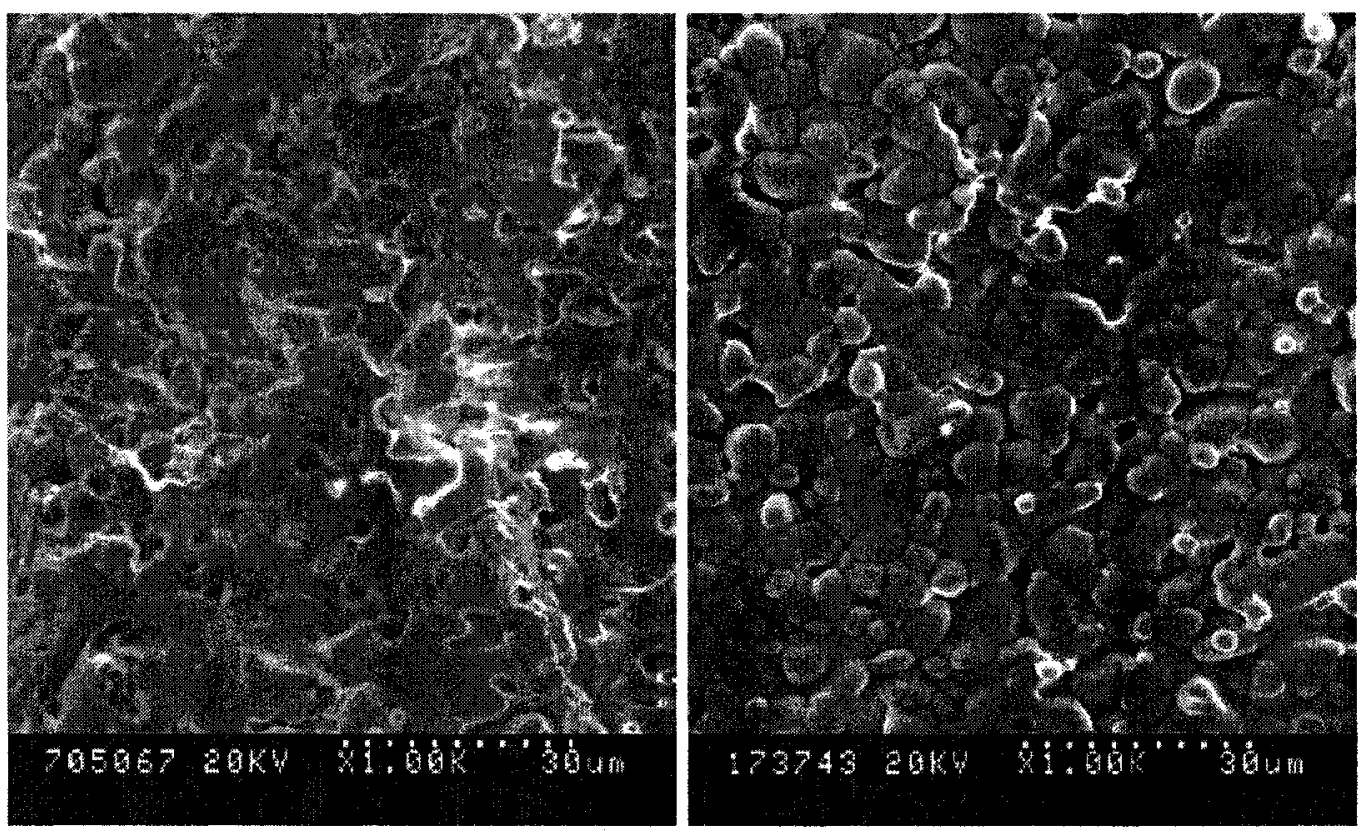

(f) $\quad 5 \mathrm{CeYSZ}$.

Figure 65 SEM micrographs of the sintered materials. Left image: fracture section; right image: sintered surface. 
The fracture section images of 3.9TaYSZ and 3.9NbYSZ in Figure 65 (a) and (b) exhibit very dense sintered ceramics and only small numbers of trapped pores were observed. The low level of porosity indicates a high density and a good sinterability of these alloyed powders. From the fracture section images of $5 \mathrm{CrYSZ}$ and $5 \mathrm{CeYSZ}$ in Figure 65 (c) and (f), and the fracture section image of 7YSZ in Figure 64, small amounts of porosity were observed. Whereas the fracture section images of 5ScYSZ and $5 \mathrm{YbYSZ}$ in Figure 65 (d) and (e) show a relatively high level of porosity with pores existing within grains and along the boundaries.

\subsubsection{Grain Size Determinations}

To measure the grain sizes, the fracture sections of the samples were polished and etched using $10 \% \mathrm{HF}$ acid for 30 minutes. The images shown in Figure 66 are from the samples 3.9TaYSZ and 3.9NbYSZ, where the grain boundaries could be observed. From Figure 66 , it can be seen that the grain size varies from $3 \mu \mathrm{m}$ to $10 \mu \mathrm{m}$ for both of the samples. Using the intercept method by drawing a straight line through about 10 grains, the average grain size was estimated to be $8.00 \mu \mathrm{m}$ for $3.9 \mathrm{TaYSZ}$; and $6.67 \mu \mathrm{m}$ for 3.9NbYSZ.

For other samples, the images obtained from the etched samples didn't show apparent grain boundaries. The grain sizes of these samples were therefore estimated from the images of the sintered surfaces shown in Figure 65 and shown in Table 8.

139 

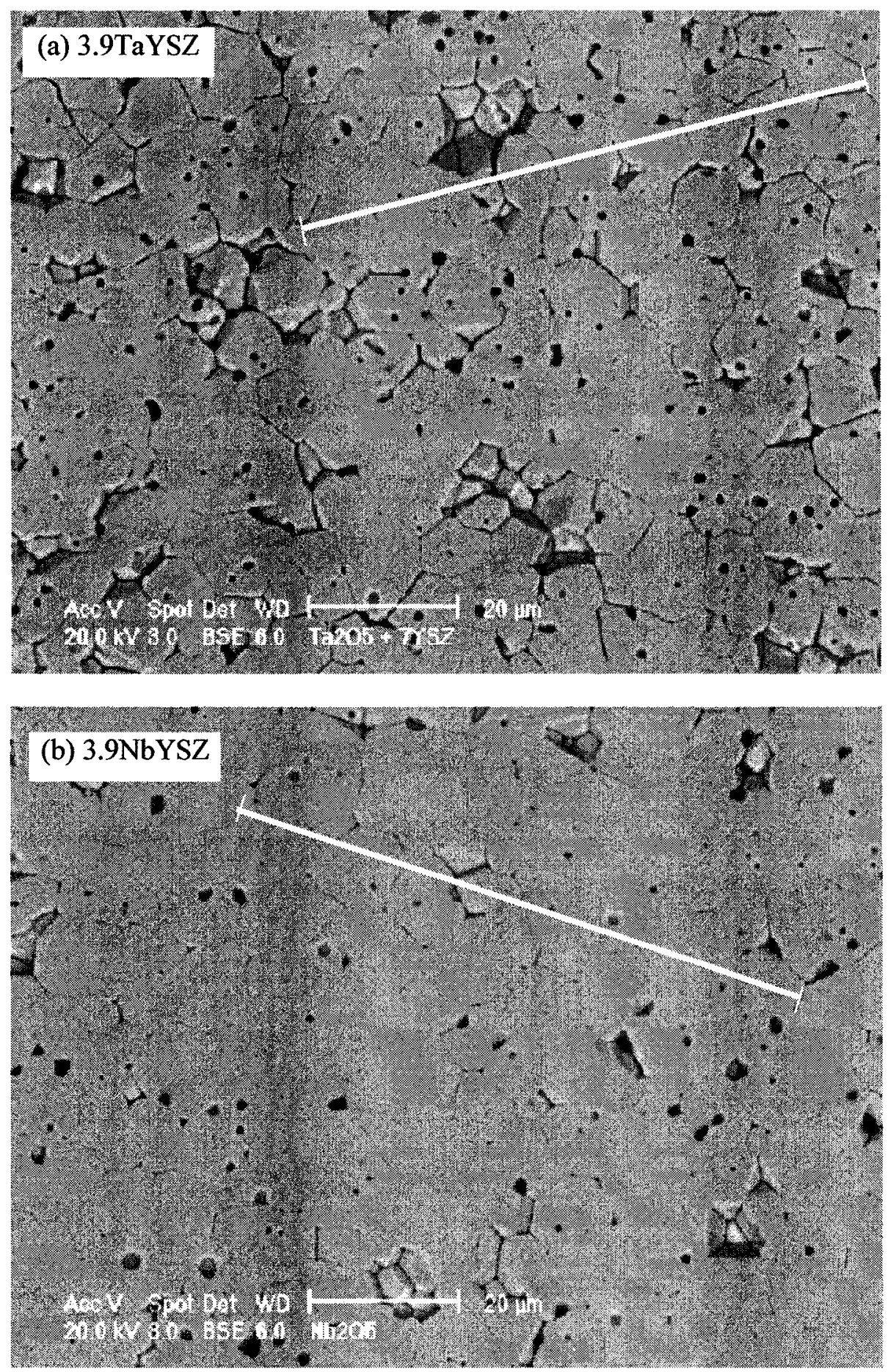

Figure 66 Estimation of grain size from the backscattered electron images of (a) 3.9TaYSZ and (b) $3.9 \mathrm{NbYSZ}$. 
Table 8 Grain size of sintered samples

\begin{tabular}{|c|c|c|}
\hline Sample ID & Grain size range $(\mu \mathrm{m})$ & Average grain size $(\mu \mathrm{m})$ \\
\hline 7 YSZ & $1 \sim 6$ & 4.29 \\
\hline 3.9 TaYSZ & $3 \sim 10$ & 8.00 \\
\hline 3.9 NbYSZ & $3 \sim 15$ & 3.75 \\
\hline 5 CrYSZ & $1 \sim 6$ & 3.00 \\
\hline 5 ScYSZ & $1 \sim 4$ & 3.00 \\
\hline $5 Y \mathrm{YbYSZ}$ & $1 \sim 6$ & 4.62 \\
\hline $5 \mathrm{CeYSZ}$ & $2 \sim 10$ & \\
\hline
\end{tabular}

\subsection{Densities of the Sintered Samples}

The bulk densities of the co-doped zirconia based ceramic samples were measured using Archimedes' method with the results shown in Table 9. To compare with theoretical densities, the lattice parameters and densities of these co-doped materials were estimated using the Aleksandrov's model [26]:

$a=2.3094\left[R_{\mathrm{Zr}}+R_{o}+\sum_{k} \frac{P_{k} \cdot M_{k} \cdot \Delta R_{k}}{100+\sum_{k} M_{k}\left(P_{k}-1\right)}\right]$

(Eq. 75)

The density is therefore evaluated as:

$\rho=\frac{Z}{0.6023 a^{3}}\left[A_{z r}+2 A_{o}+\sum_{k} \frac{P_{k} \cdot M_{k} \cdot\left[\Delta A_{k}+A_{o}\left(\begin{array}{c}\left.P_{o k}-2\right) \\ P_{k}\end{array}\right]\right.}{100+\sum_{k} M_{k}\left(P_{k}-1\right)}\right]$

(Eq. 76) 
where $R_{z r}$ and $R_{o}$ are the ionic radii of $\mathrm{Zr}^{4+}$ and $\mathrm{O}^{2-} ; A_{z r}$ and $A_{o}$ are the gram atomic weight of $\mathrm{Zr}^{4+}$ and $\mathrm{O}^{2-}$, respectively; $\Delta R_{k}$ is the difference between the ionic radius of the $k$ th stabilizing element and that of $\mathrm{Zr}^{4+} ; \Delta A_{k}$ is the difference between the gram atomic weight of the $k$ th stabilizing element and that of $\mathrm{Zr} . P_{k}$ is the number of ions per molecule of the stabilizing element; $P_{o k}$ is the number of oxygen ions per molecule in the stabilizer; and $M_{k}$ is the mole percentage of the $k$ th stabilizer.

The theoretical density as well as the relative density of each sintered material were estimated and listed in Table 9. The calculated results are fully consistent with those of both tetragonal and cubic structures. For the monoclinic structure, the values calculated using the equation above are higher than the measured density [26], since the Aleksandrov model is based on an ideal fluorite structure and the packing of ions. The monoclinic phase was expected to have lower density due to its larger lattice parameters (thus the larger unit cell volume) than those of tetragonal and cubic structures. As indicated by XRD patterns, the samples of both 3.9TaYSZ and 3.9NbYSZ in the present study showed monoclinic structures which is thought to be due to the annihilation of oxygen vacancies. For the comparison, the measured density published in Ref. [26] for the monoclinic $\mathrm{ZrO}_{2}$ was adopted in our study.

The data shown in Table 9 indicates that the relative densities of the sintered 3.9TaYSZ and $3.9 \mathrm{NbYSZ}$ samples have reached as high as $95 \%$ and exhibit very good sinterability. The 7YSZ, 5CrYSZ and 5CeYSZ samples have more than $90 \%$ relative densities. The relative densities of $5 \mathrm{ScYSZ}$ and $5 \mathrm{YbYSZ}$ samples are only $89 \%$ and $84 \%$, which suggests that they exhibit poor sinterability. These data are also confirmed by SEM 
images showing the microstructures as well as the porosity calculation from the image analyzer which are discussed in the following sections.

Table 9 Measured density, theoretical density and relative density

\begin{tabular}{|l|l|l|l|l|}
\hline Sample ID & $\begin{array}{l}\text { Measured } \\
\text { density }\left(\mathrm{g} / \mathrm{cm}^{3}\right)\end{array}$ & $\begin{array}{l}\text { Calculated cubic lattice } \\
\text { parameter } a(\mathrm{~nm})\end{array}$ & $\begin{array}{l}\text { Theoretical } \\
\text { density }\left(\mathrm{g} / \mathrm{cm}^{3}\right)\end{array}$ & Relative \\
density $(\%)$
\end{tabular}

* The theoretical densities for 3.9TaYSZ and 3.9NbYSZ showing in the table were calculated using the volume of monoclinic structure of $\mathrm{ZrO}_{2}$., where $\mathrm{V}_{\mathrm{ZrO} 2}(\mathrm{~m})=0.14088$ $\mathrm{nm}^{3}[123]$

\subsection{Porosities in the Sintered Samples}

The volume fractions of porosity and average pore sizes inside the sintered ceramics were measured using image analyzer and the results are given in Table 10. For comparison, the volume fractions of porosity $P$ within the sintered materials were also evaluated using density measurements and the following equation: 


$$
P=\frac{\rho_{\text {theoretical }}-\rho_{\text {meausred }}}{\rho_{\text {theorenical }}}
$$

The volume fraction of porosity calculated from the image analyzer was based on the assumption that all the porosities were spherical, and therefore the volume fraction was considered to be the same as the area fraction. In fact, most of the pores were not spherical, as shown in Figure 66, and this might cause some errors. On the other hand, the porosity volume fraction evaluated from (Eq. 77$)$ may also be subject to errors: for example, the measured density will be subject to random experimental errors, and the theoretical densities were based on the cubic structure and the lattice parameters estimated from the dopant concentrations. Considering these two situations, thus, to reduce the error, the average of the two fractions are also listed in Table 10.

Table 10 Pore volume fractions

\begin{tabular}{|c|c|c|c|}
\hline Sample ID & Vol.\% (image analyzer) & Vol.\% (density measurement) & Average Vol.\% \\
\hline 7 YSZ & 18.30 & 8.09 & 13.19 \\
\hline 3.9 TaYSZ & 12.71 & 4.90 & 8.81 \\
\hline $3.9 \mathrm{NbYSZ}$ & 4.04 & 5.32 & 4.68 \\
\hline 5CrYSZ & 14.77 & 9.48 & 12.13 \\
\hline 5ScYSZ & 11.07 & 11.07 & 13.36 \\
\hline 5YbYSZ & 15.92 & 9.00 & 14.12 \\
\hline 5CeYSZ & 7.10 & 3.50 & 5.30 \\
\hline
\end{tabular}




\subsection{Elemental Composition Analysis}

The homogeneity of the doped materials distributed throughout the sintered materials was examined by elemental mapping using EDX. Figure 67 and Figure 68 show typical SE image of analyzed areas and the elemental maps of $\mathrm{Zr}, \mathrm{Ce}$ and $\mathrm{Sc}$, respectively. It can be seen that both $\mathrm{Ce}$ and $\mathrm{Sc}$ are distributed uniformly throughout the doped materials.
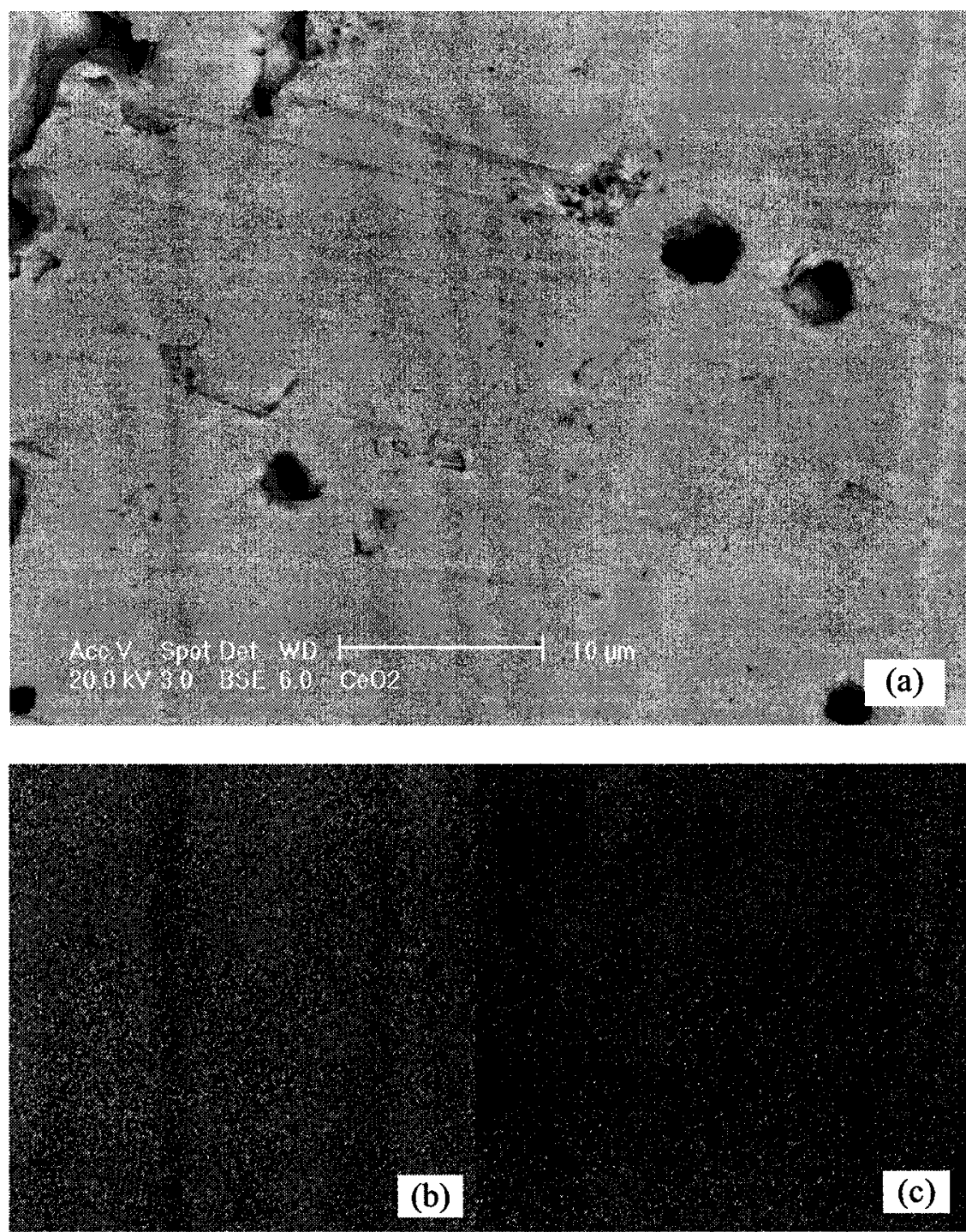

Figure 67 EDX mapping of sintered 5CeYSZ. (a) SE image of the area being mapped;

(b) Zr mapping; and (c) Ce mapping. 

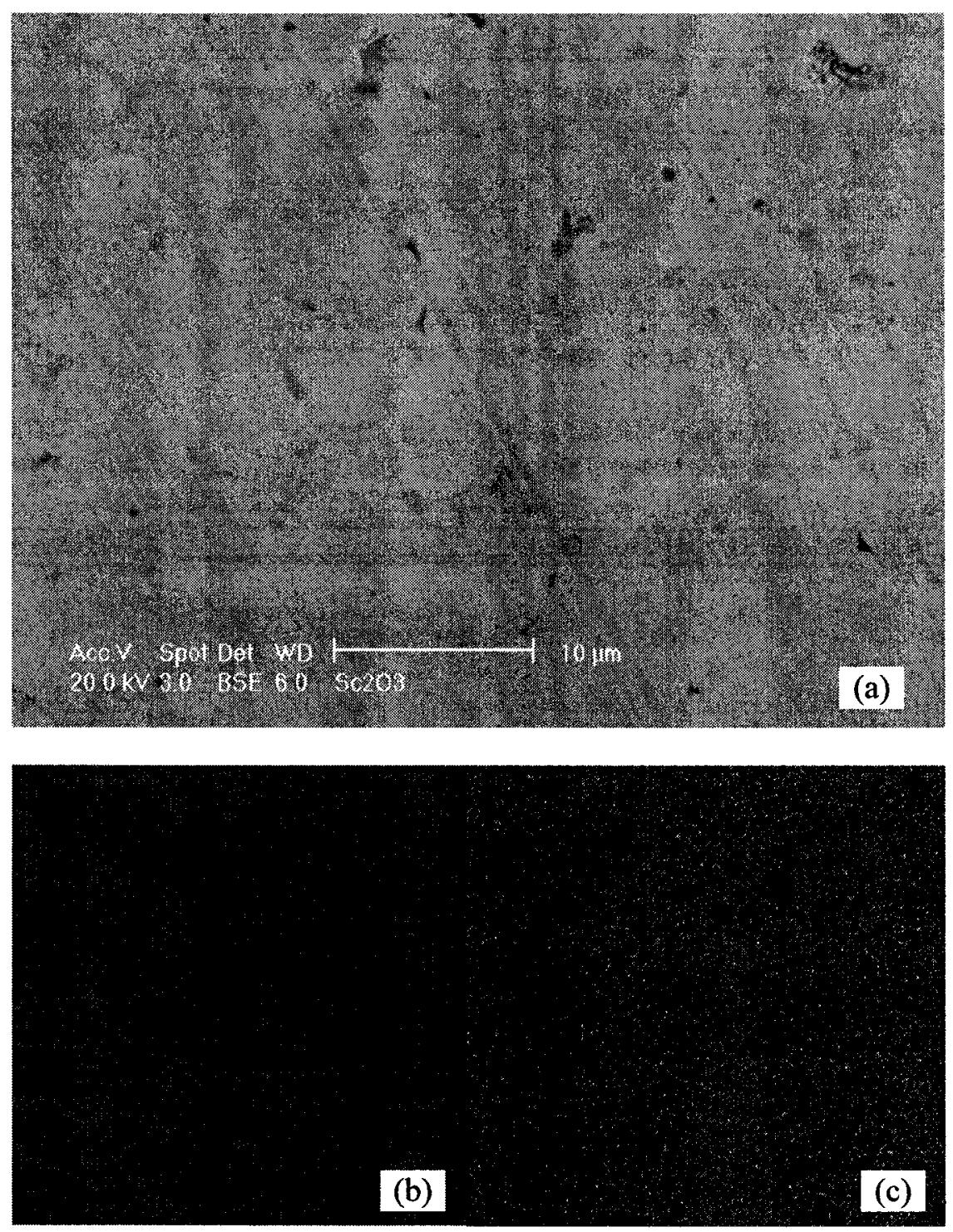

Figure 68 EDX mapping of sintered 5ScYSZ. (a) SE image of the area being mapped; (b) zirconium mapping; and (c) scandium mapping.

The quantitative element compositional analysis was conducted for each doped 7YSZ sample, and the composition and the spectra of the relative dopant at different spots in each sample were measured and are shown in Figure 69. From these BEI images, some small dark particles were observed to be dispersed within the area of some large grains. 
The size of the dark particles varies from $0.5 \mu \mathrm{m}$ to $2 \mu \mathrm{m}$, depending on the dopant cations. The total area fraction of these small dark particles was estimated to be less than $5 \%$.

To examine the element distribution, 8 spots were selected to do the element compositional analysis for each doped material, as labeled in Figure 69. In all these figures (Figure 69 (a) $\sim(\mathrm{f})$ ), the areas indicated by the numbered spots are equivalent in each figure. For example, spot \#4 in Figure 69 (a) is similar to spot \#4 in Figure 69 (b). Spots \#1, \#2, and \#3 are located in the center of the randomly picked grains; spots \#4 and \#5 lie in the center of two dark small particles; and spots \#6, \#7, and \#8 are in the areas from the grain boundary or the edge of the dark particles to the center of grains, with about $1 \mu \mathrm{m}$ distance between each two spots.

For the quantitative element analysis, it should be noted that

1. Since the mass absorption coefficients for light element such as oxygen with lowenergy $\mathrm{x}$-rays $(<0.7 \mathrm{KeV})$ are very large, which result in inaccurate concentration of oxygen detected at the peak $0.5 \mathrm{KeV}$. Thus, it is very hard to obtain accurate atomic fraction of each element. To obtain the accurate dopants composition, in the following discussion, only the compositions of the dopant cations are considered.

2. At lower energy range (below $3 \mathrm{keV}$ ), the separations of the members of the $\mathrm{K}, \mathrm{L}$, or $\mathrm{M}$ families are so small that the $\mathrm{x}$-ray peaks are not resolved with an EDX system. Thus the elements $\mathrm{Y}-\mathrm{L} \alpha(1.92 \mathrm{KeV})$ and $\mathrm{Zr}-\mathrm{L} \alpha(2.0 \mathrm{KeV})$ couldn't be separated completely and the quantitative analysis between $\mathrm{Y}$ and $\mathrm{Zr}$ might not be 147 
accurate.
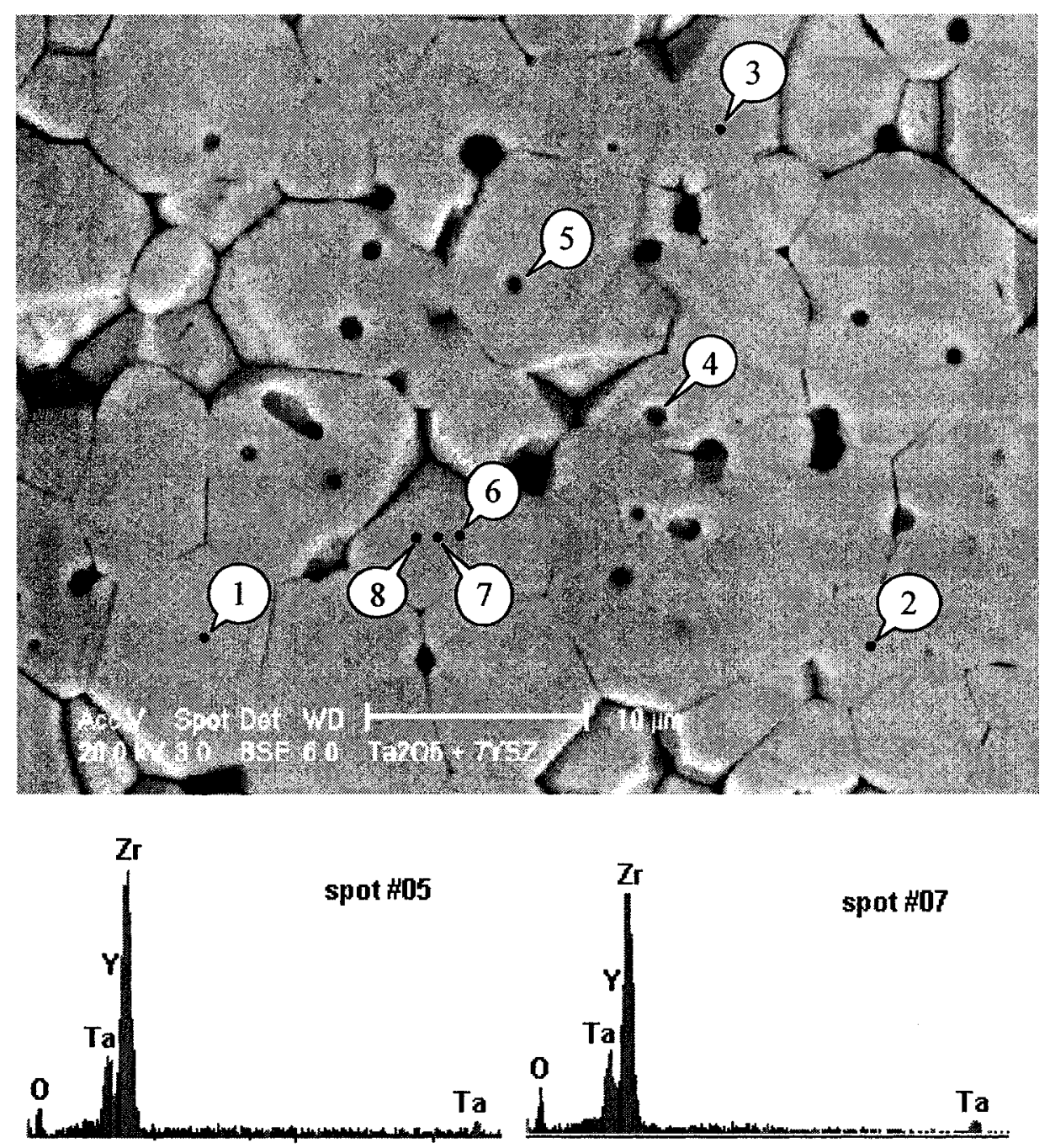

spot $\$ 07$

\begin{tabular}{|l|l|l|l|l|l|l|l|l|}
\hline Element & $\# 1$ & $\# 2$ & $\# 3$ & $\# 4$ & $\# 5$ & $\# 6$ & $\# 7$ & $\# 8$ \\
\hline $\mathrm{Y} \mathrm{(mol \% )}$ & 11.41 & 11.47 & 11.22 & 12.69 & 12.04 & 12.89 & 12.28 & 13.04 \\
\hline $\mathrm{Zr}$ (mol\%) & 81.33 & 81.33 & 81.77 & 78.74 & 79.41 & 78.93 & 80.59 & 79.96 \\
\hline $\mathrm{Ta} \mathrm{(mol \% )}$ & 7.25 & 7.20 & 7.03 & 8.57 & 8.55 & 8.18 & 7.13 & 6.99 \\
\hline
\end{tabular}

(a) 3.9TaYSZ 

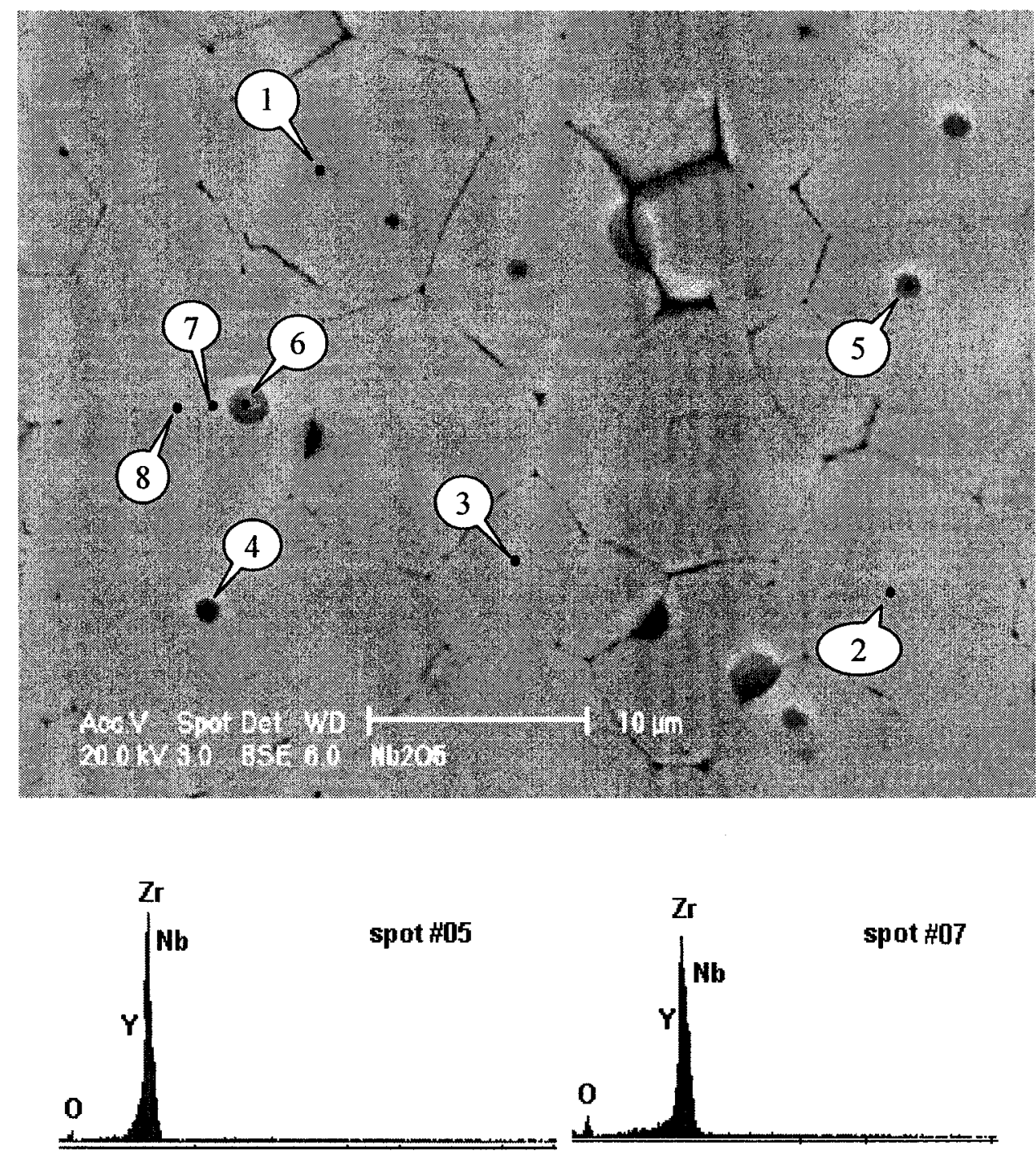

\begin{tabular}{|l|l|l|l|l|l|l|l|l|}
\hline Element & $\# 1$ & $\# 2$ & $\# 3$ & $\# 4$ & $\# 5$ & $\# 6$ & $\# 7$ & $\# 8$ \\
\hline $\mathrm{Y} \mathrm{(mol \% )}$ & 11.15 & 8.54 & 8.98 & 9.39 & 9.72 & 10.94 & 8.80 & 10.71 \\
\hline $\mathrm{Zr} \mathrm{(mol \% )}$ & 75.54 & 79.49 & 79.48 & 77.815 & 77.96 & 77.84 & 79.55 & 77.43 \\
\hline $\mathrm{Nb}(\mathrm{mol} \%)$ & 13.32 & 11.97 & 11.54 & 12.79 & 12.32 & 11.215 & 11.65 & 11.86 \\
\hline
\end{tabular}

(b) $3.9 \mathrm{NbYSZ}$ 

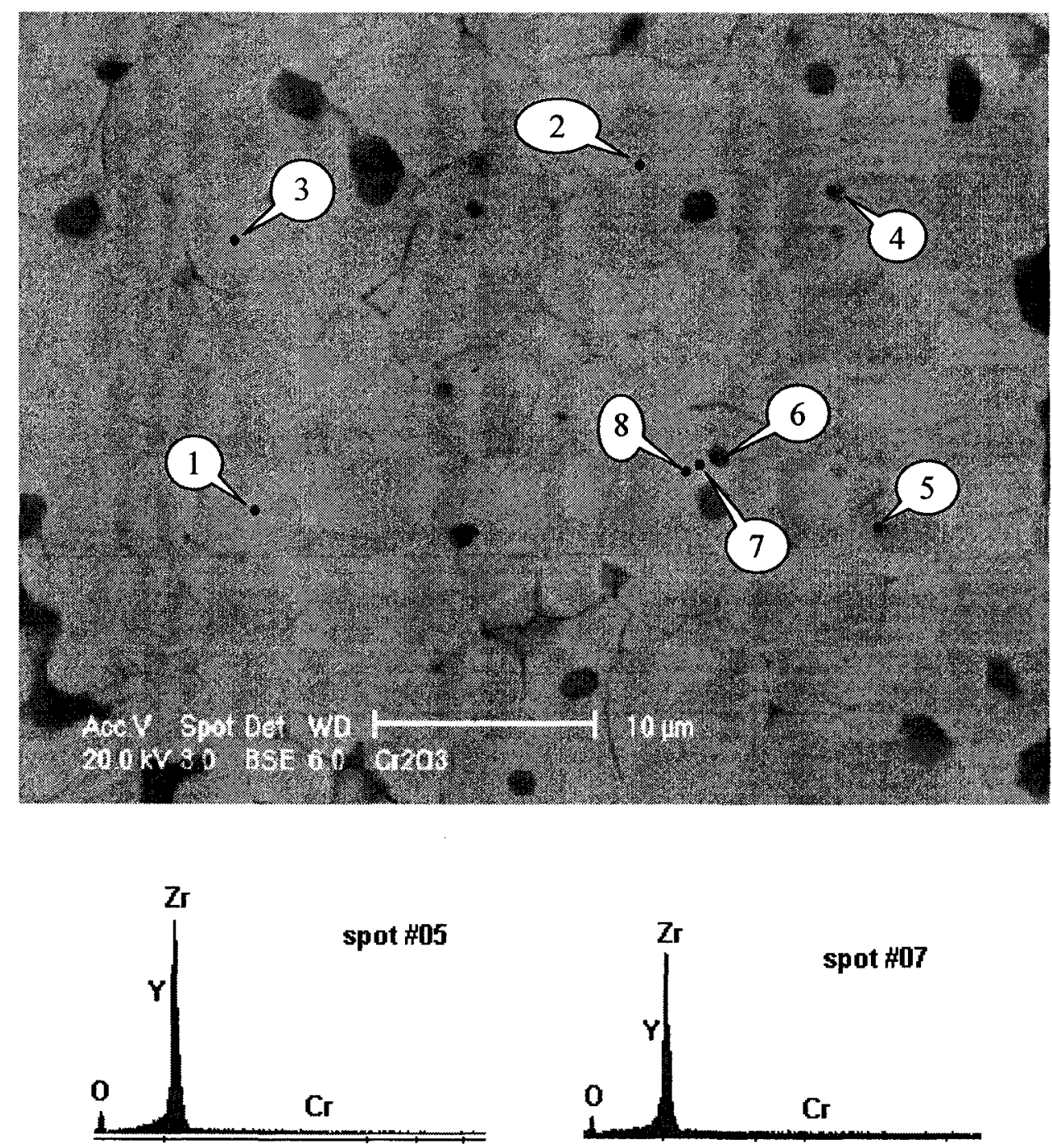

\begin{tabular}{|l|l|l|l|l|l|l|l|l|}
\hline Element & $\# 1$ & $\# 2$ & $\# 3$ & $\# 4$ & $\# 5$ & $\# 6$ & $\# 7$ & $\# 8$ \\
\hline $\mathrm{Y}(\mathrm{mol} \%)$ & 0.00 & 6.78 & 32.55 & 29.60 & 32.32 & 31.165 & 34.93 & 33.80 \\
\hline $\mathrm{Zr}(\mathrm{mol} \%)$ & 99.55 & 92.64 & 67.00 & 70.14 & 66.52 & 67.93 & 64.59 & 65.76 \\
\hline $\mathrm{Cr}(\mathrm{mol} \%)$ & 0.45 & 0.58 & 0.46 & 0.87 & 1.16 & 0.90 & 0.47 & 0.44 \\
\hline
\end{tabular}

(b) $5 \mathrm{CrYSZ}$ 

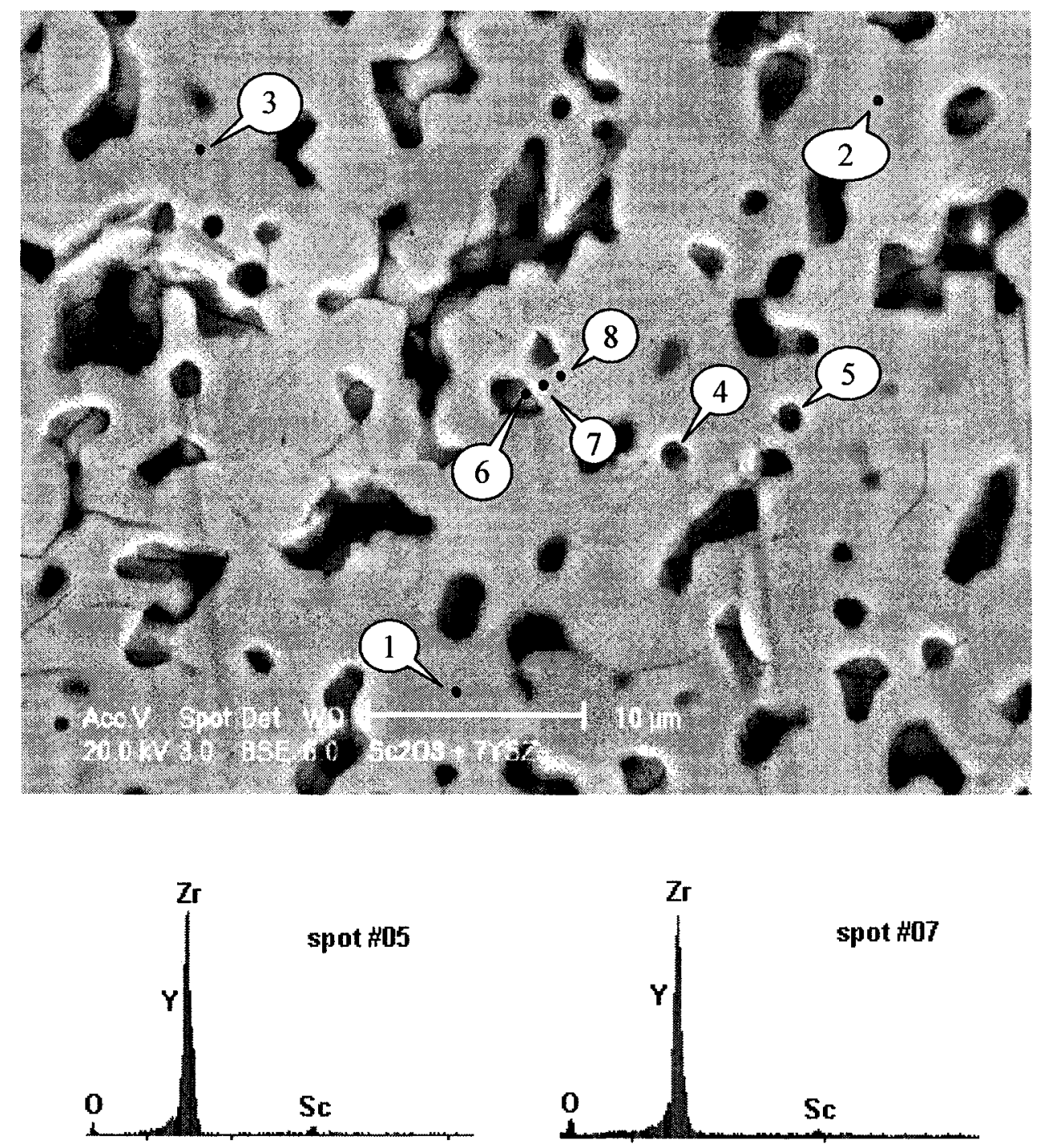

\begin{tabular}{|l|l|l|l|l|l|l|l|l|}
\hline Element & $\# 1$ & $\# 2$ & $\# 3$ & $\# 4$ & $\# 5$ & $\# 6$ & $\# 7$ & $\# 8$ \\
\hline $\mathrm{Y}(\mathrm{mol} \%)$ & 9.80 & 9.58 & 11.47 & 10.60 & 11.42 & 12.36 & 11.00 & 11.52 \\
\hline $\mathrm{Zr}(\mathrm{mol} \%)$ & 85.07 & 84.89 & 84.02 & 83.86 & 83.135 & 82.27 & 83.86 & 83.40 \\
\hline $\mathrm{Sc}(\mathrm{mol} \%)$ & 5.13 & 5.53 & 4.52 & 5.54 & 5.44 & 5.37 & 5.15 & 5.08 \\
\hline
\end{tabular}

(c) $\quad 5 \mathrm{ScYSZ}$ 

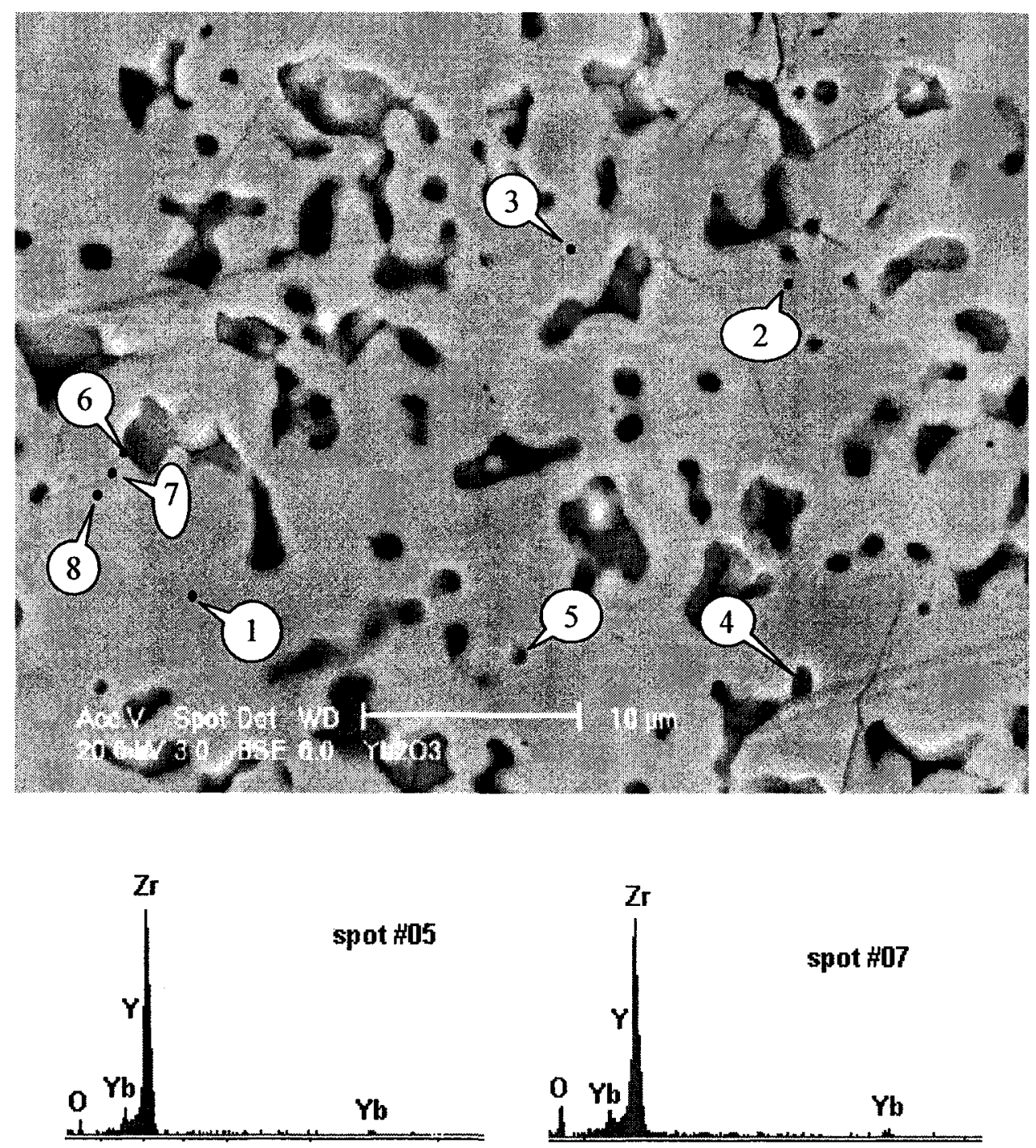

\begin{tabular}{|l|l|l|l|l|l|l|l|l|}
\hline Element & $\# 1$ & $\# 2$ & $\# 3$ & $\# 4$ & $\# 5$ & $\# 6$ & $\# 7$ & $\# 8$ \\
\hline $\mathrm{Y}(\mathrm{mol} \%)$ & 28.70 & 26.29 & 22.48 & 28.53 & 23.19 & 27.07 & 19.98 & 27.40 \\
\hline $\mathrm{Zr}(\mathrm{mol} \%)$ & 68.12 & 69.81 & 75.30 & 66.46 & 70.80 & 67.65 & 75.29 & 68.00 \\
\hline $\mathrm{Yb} \mathrm{(mol \% )}$ & 3.19 & 3.73 & 2.22 & 5.01 & 6.01 & 5.28 & 4.73 & 4.60 \\
\hline
\end{tabular}

(e) 5 YbYSZ 

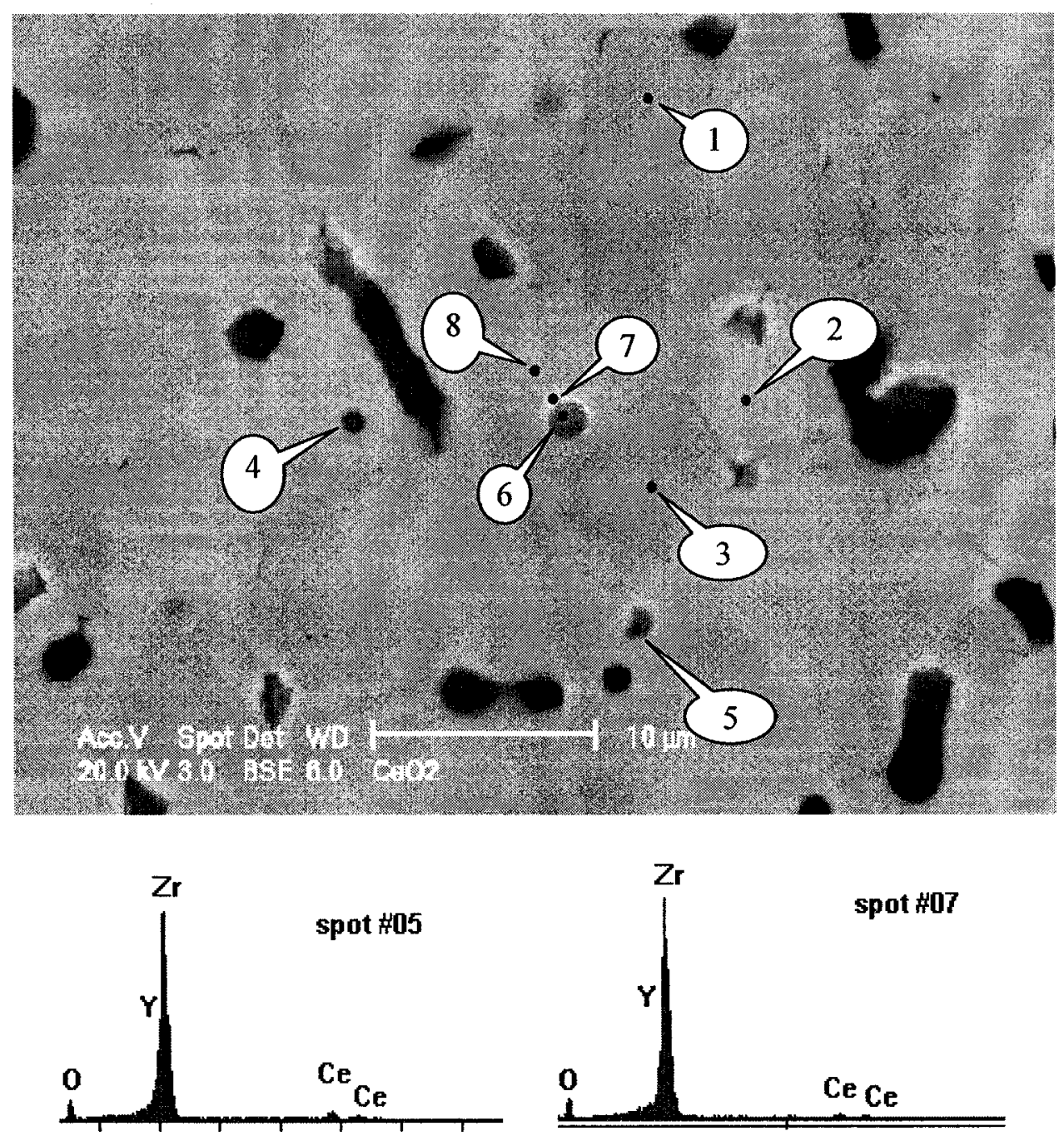

\begin{tabular}{|l|l|l|l|l|l|l|l|l|}
\hline Element & $\# 1$ & $\# 2$ & $\# 3$ & $\# 4$ & $\# 5$ & $\# 6$ & $\# 7$ & $\# 8$ \\
\hline $\mathrm{Y} \mathrm{(mol \% )}$ & 5.39 & 14.43 & 6.38 & 11.71 & 13.42 & 14.96 & 15.03 & 15.10 \\
\hline $\mathrm{Zr}(\mathrm{mol} \%)$ & 91.34 & 80.19 & 90.17 & 81.28 & 79.78 & 78.15 & 79.19 & 79.49 \\
\hline $\mathrm{Ce}(\mathrm{mol} \%)$ & 3.27 & 5.38 & 3.45 & 7.02 & 6.80 & 6.89 & 5.78 & 5.40 \\
\hline
\end{tabular}

(f) 5 CeYSZ

Figure 69 BEI images and element compositional analysis of doped materials: (a) 3.9TaYSZ; (b) 3.9NbYSZ; (c) 5CrYSZ; (d) 5ScYSZ; (e) 5YbYSZ; and (f) 5CeYSZ. 
Comparing the dopant compositions measured at each spot, it is found that the dopant cations preferred to lie in the positions near the $\mathrm{Y}$ cations. That is, if the concentration of $\mathrm{Y}$ is high, then the concentration of the dopant cations is high as well. On the opposite, at positions with lower content of $\mathrm{Y}$, the content of the dopant cations is also lower.

Within the area, such as spots \#4 and \#5, of small dark particles, the contents of both $\mathrm{Y}$ and dopant cations are higher than those within other areas for the 3.9TaYSZ, 3.9NbYSZ, 5YbYSZ and 5CeYSZ samples; this indicates that within these dark particles, more oxygen vacancies might have been created. On the other hand, in the area close to the edge of dark particles or grain boundaries, such as spot \#6, the contents of both $Y$ and the dopant cations are higher than those in the center of large grains and lower than those within the dark particles.

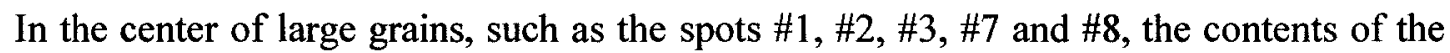
elements for the 3.9TaYSZ, 3.9NbYSZ, and 5ScYSZ samples are very close and exhibit a homogeneous distribution for each relative element. For the 5YbYSZ and 5CeYSZ samples, the contents of elements within these areas are somewhat dissimilar and have two slightly different levels of concentration. The images of $5 \mathrm{YbYSZ}$ and $5 \mathrm{CeYSZ}$ samples show some grains with shadow-like appearances. The compositions of $\mathrm{Yb}$ or $\mathrm{Ce}$ and $\mathrm{Y}$ within the shadowed grains on the images of $5 \mathrm{YbYSZ}$ and $5 \mathrm{CeYSZ}$ are slightly lower that those within the light grains; in contrast, a higher composition of $\mathrm{Zr}$ is observed within the shadowed grains than within the light grains. This is a result of their diffusion coefficients: the ionic radii of the dopants $\mathrm{Ta}, \mathrm{Nb}$ and $\mathrm{Sc}$ are smaller than that of $\mathrm{Zr}$ and therefore could diffuse faster when the samples were cooled down. On the other 
hand, due to the relatively large ionic radii of the dopants $\mathrm{Yb}$ and $\mathrm{Ce}$, they would be expected to diffuse slower. Thus, it appears that with the same crystal structure, but slightly different compositions, two phases were formed.

For 5CrYSZ samples, unfortunately, the $\mathrm{Cr}^{3+}$ dopant content measured by EDX from the BEI images is very low. Thus, the results for 5 CrYSZ couldn't give useful information. It seems that the $\mathrm{Cr}^{3+}$ ions had evaporated during long time high temperature sintering since the $\mathrm{Cr}_{2} \mathrm{O}_{3}$ might be converted into $\mathrm{CrO}_{3}$, which has a melting point of only $197^{\circ} \mathrm{C}$.

\subsection{Phases Identification}

The properties of the sintered zirconia-based materials are determined by both crystal structures and microstructures. At equilibrium, three structures may appear for zirconiabased ceramics: monoclinic, tetragonal and cubic phases, as explained earlier. The phases present at room temperature depend on the content of dopants and heat treatment conditions. To identify the phases present at room temperature for each doped 7YSZ material, the x-ray diffraction technique was used. By analyzing the XRD patterns, the fraction of the phase present, lattice parameters, as well as the equivalent yttria content of each sintered sample were estimated.

The XRD patterns of all the samples studied are shown in Figure $70 \sim$ Figure 74 . From the XRD peaks reflected from (111) plane for the cubic or tetragonal structure and the peaks reflected from $m(\overline{1} 11)$ and $m(111)$ planes for the monoclinic structure, the fraction of monoclinic phase, if any, was calculated for each sample using (Eq. 62) and the results are listed in Table 11. The lattice constant $a$ for the cubic phase and the axial 
$c / a$ ratio for the tetragonal phase were calculated from the relationships between the lattice parameter and the interplanar spacing ((Eq. 63) and (Eq. 64)) are also listed in Table 11.

\subsection{1. $7 Y S Z$ and $5 C r Y S Z$}

The XRD patterns of the 7YSZ sample exhibiting both monoclinic and cubic phases are shown in Figure 70.
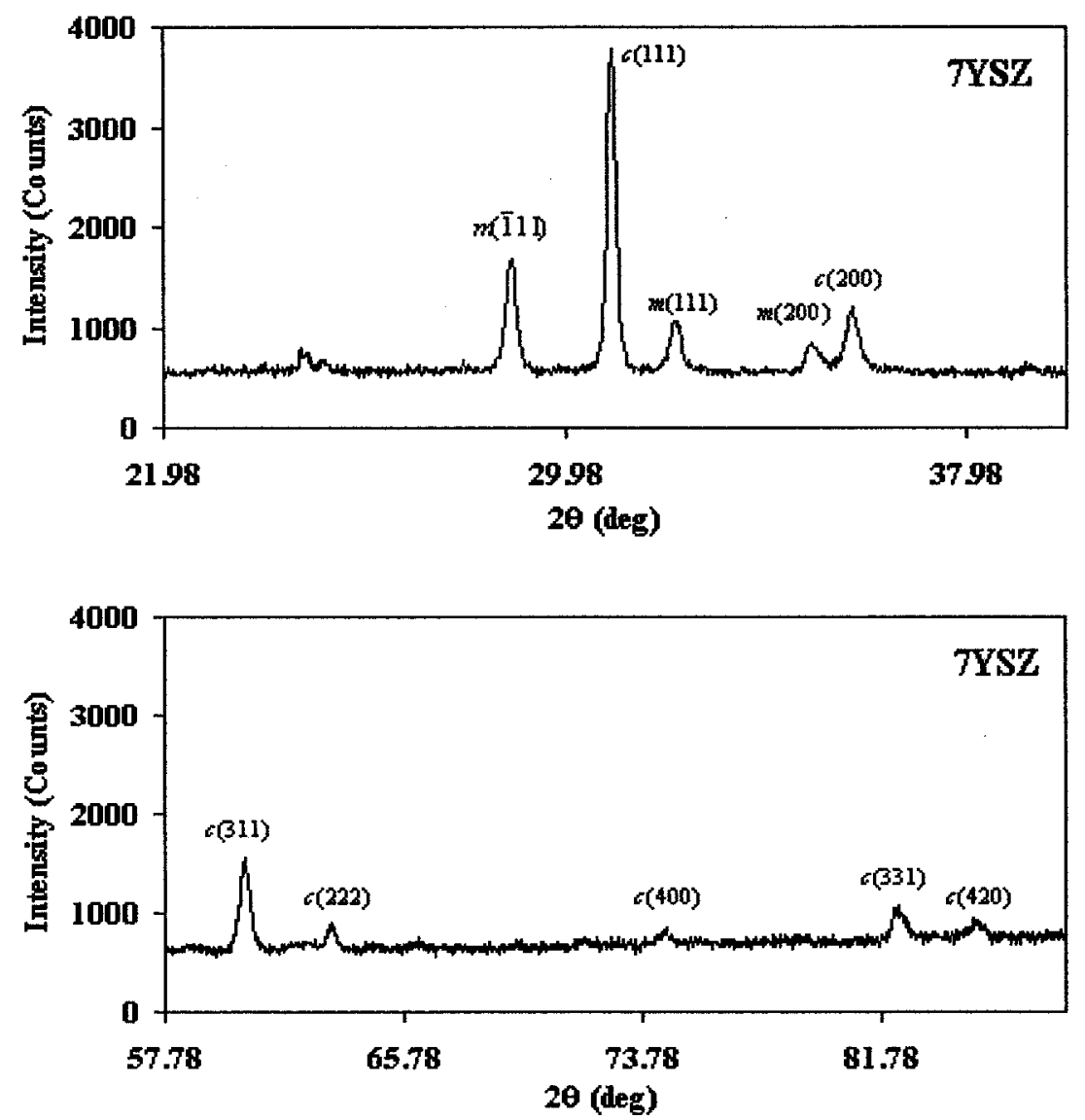

Figure 70 XRD patterns of the sintered 7YSZ sample.

From the patterns, it can be seen that the 7YSZ at room temperature consists of 156 
monoclinic and cubic phases. The fraction of the monoclinic phase was calculated using (Eq. 62) to be about $45.96 \mathrm{~mol} \%$. Due to the insufficient time during cooling to achieve complete equilibrium, the actual phases measured from XRD might include small amounts of tetragonal phase, which couldn't be observed due to the limitation of the equipment.

The XRD patterns of the 5 CrYSZ were very similar to that of $7 \mathrm{YSZ}$, as shown in Figure 71.
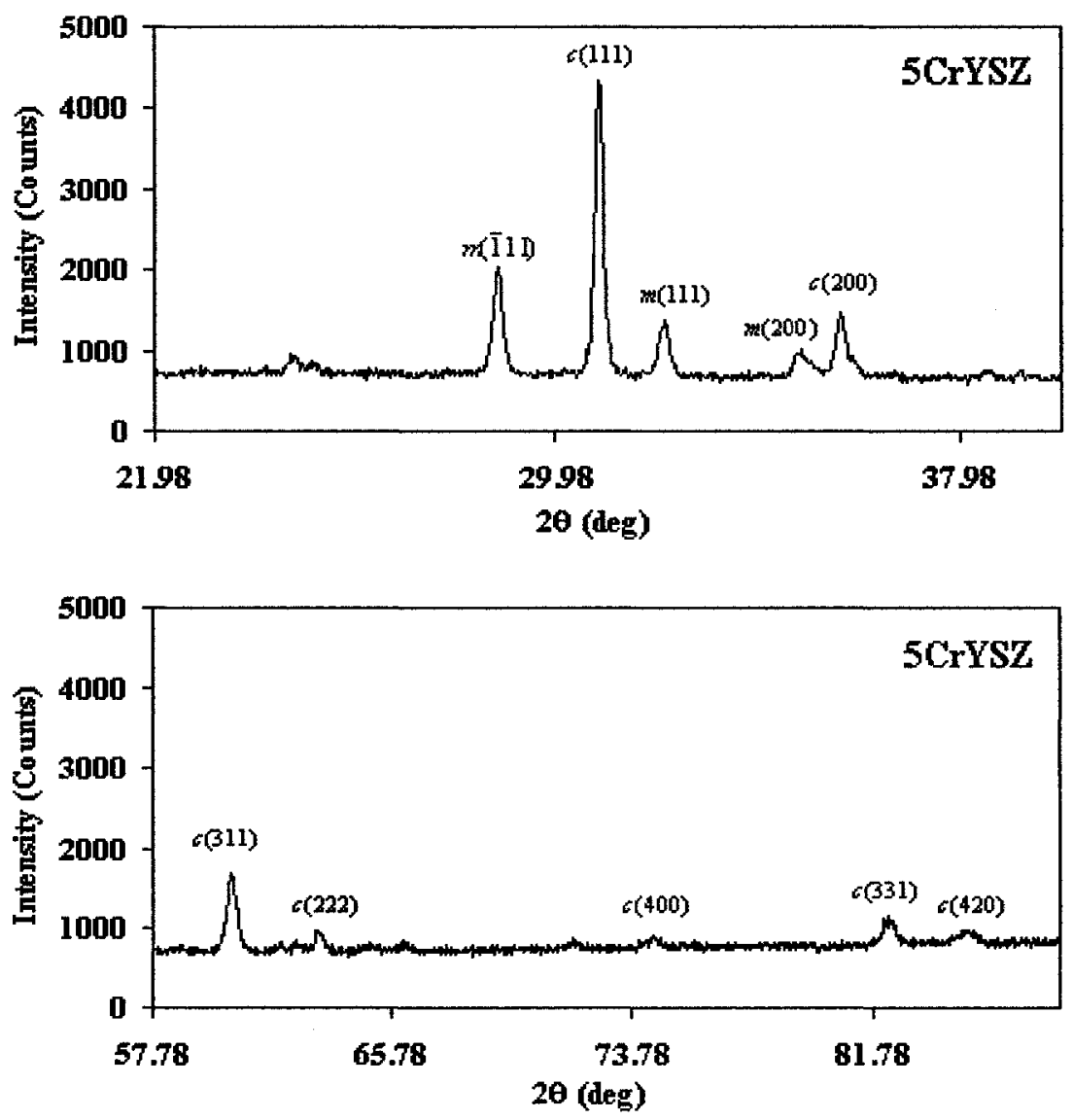

Figure 71 XRD patterns of the sintered 5CrYSZ sample.

The XRD results indicate that the 5 CrYSZ sample contains monoclinic phase with a 157 
concentration of $51.52 \mathrm{~mol} \%$ and cubic phase with a concentration of $48.48 \mathrm{~mol} \%$ at room temperature. The larger amount of monoclinic phase than in 7YSZ might be due to the small ionic radius of $\mathrm{Cr}$ cations, which played a role of de-stabilization instead of creating more oxygen vacancies to stabilize the cubic phase. From the previous EDS element analysis for $5 \mathrm{CrYSZ}$, it has been seen that only a very small amount of $\mathrm{Cr}$ cation was detected. Thus, the chemical composition of $5 \mathrm{CrYSZ}$ is very close to that of $7 \mathrm{YSZ}$, and its XRD pattern is also very similar to that of 7YSZ.

\subsection{TaYSZ and 3.9NbYSZ}

The XRD patterns of the samples of 3.9TaYSZ and 3.9NbYSZ are shown in Figure 72 (a) and (b). These two samples exhibit almost 100\% monoclinic phase except for a small peak appearing at the $2 \theta$ of 31.02 degree in the patterns of $3.9 \mathrm{NbYSZ}$ sample, as shown in Figure 72 (b). This is thought to be due to the reflection from the (111) plane of the tetragonal structure and which has a fraction of about $3.48 \mathrm{~mol} \%$. This small amount of tetragonal phase might be retained when the crystal was cooled down from high temperature to room temperature, due to the incomplete phase transformation.

The almost $100 \%$ transformation from tetragonal to monoclinic phase when these two doped materials were cooled down from high temperature to room temperature was due to the very low content of oxygen vacancies. In addition, both $\mathrm{Ta}^{5+}$ and $\mathrm{Nb}^{5+}$ cations had smaller ionic radii than that of $\mathrm{Zr}^{4+}$ and thus had an opposite effect on the stabilizing of tor $\mathrm{c}-\mathrm{ZrO}_{2}$. 

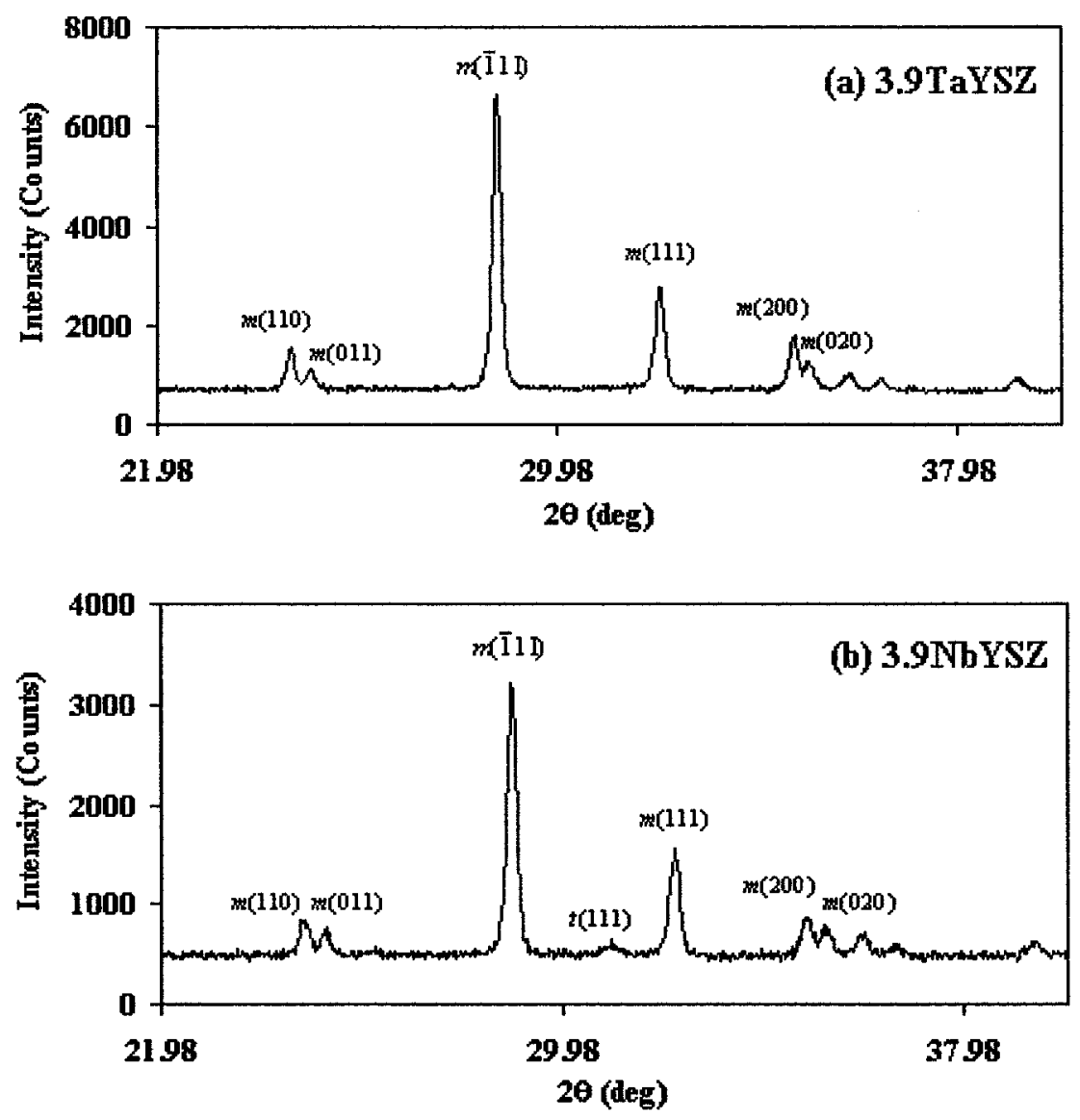

Figure 72 XRD patterns of the sintered samples (a) 3.9TaYSZ; (b) 3.9NbYSZ.

\subsubsection{SeYSZ and 5YbYSZ}

For the 5ScYSZ sample, both the cubic phase and the monoclinic phase were observed from the XRD patterns in Figure 73 (a), with a monoclinic phase fraction of only about $5.73 \mathrm{~mol} \%$ present at room temperature. For the sample $5 \mathrm{YbYSZ}$, no monoclinic phase was observed from its XRD pattern, as shown in Figure 73 (b).

Though the oxygen vacancy concentrations created within the two samples $5 \mathrm{ScYSZ}$ and 5 YbYSZ were very similar, the differences in the actual phases present at room 
temperatures might be due to their different ionic radii. For the 5ScYSZ sample, the smaller ionic radius of $\mathrm{Sc}^{3+}$ ion compared to $\mathrm{Yb}^{3+}$ ion would have less effect on stabilization.
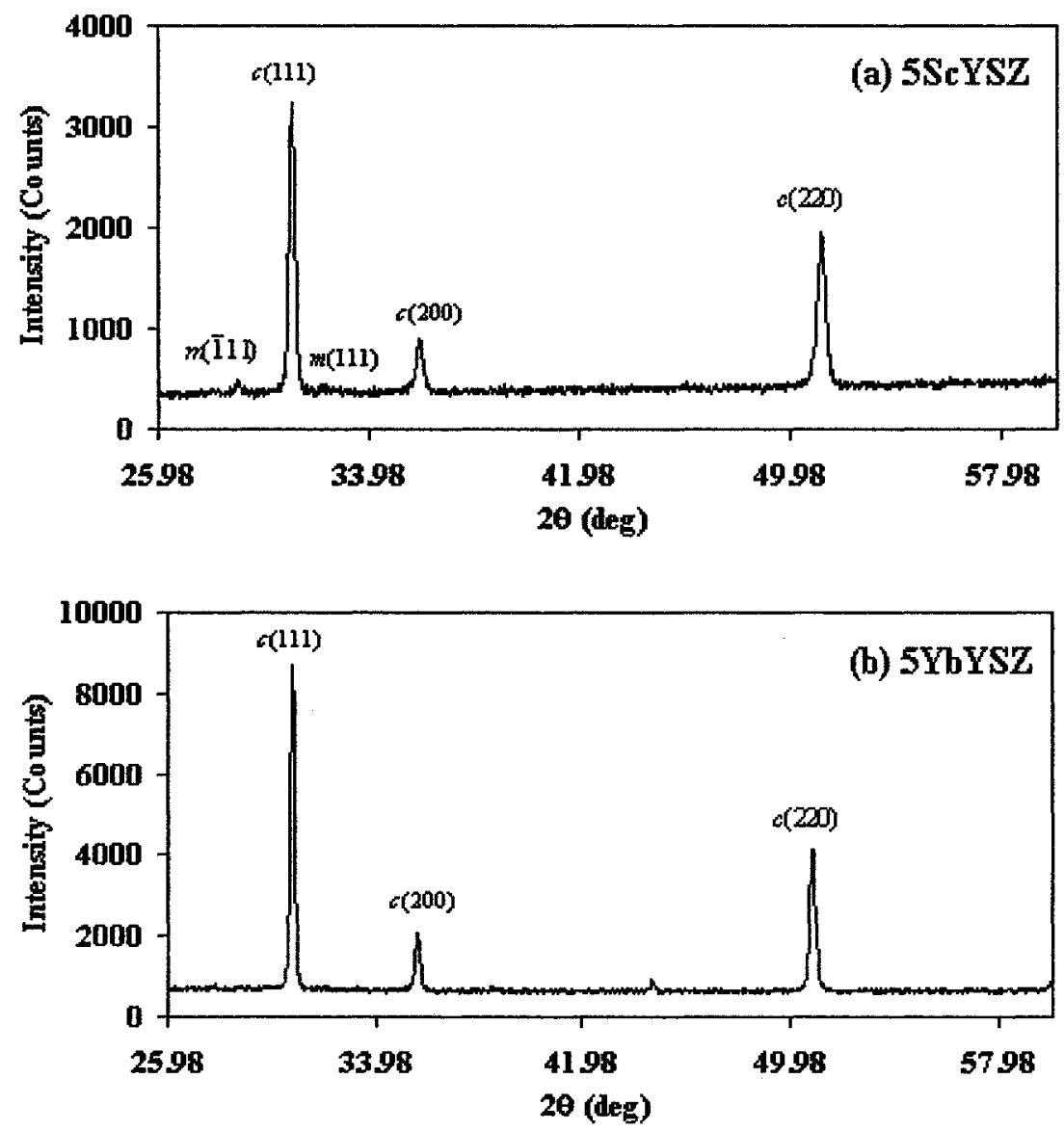

Figure 73 XRD patterns of the sintered samples (a) 5ScYSZ; (b) 5YbYSZ.

\subsubsection{CeYSZ}

The XRD pattern for $5 \mathrm{CeYSZ}$ was different from those of the other samples, as shown in Figure 74. It indicated the presence of a very small amount of monoclinic phase of only about $2.49 \mathrm{~mol} \%$, the rest $(97.51 \mathrm{~mol} \%)$ most probably consisted of a mixture of tetragonal and cubic phases. The grains shown in the backscattered electron image in 160 
Figure 69 (f) and the EDS analysis for this sample also exhibited two different dopant and yttrium contents. The grains having lower contents are thought to be the tetragonal phase and the grains having higher contents are thought to be the cubic phase. Compared with the dopants $\mathrm{Sc}_{2} \mathrm{O}_{3}$ and $\mathrm{Yb}_{2} \mathrm{O}_{3}$, the $\mathrm{CeO}_{2}$ exhibited a lower effect on stabilizing cubic phase $7 \mathrm{YSZ}$ than the $\mathrm{Sc}_{2} \mathrm{O}_{3}$ and $\mathrm{Yb}_{2} \mathrm{O}_{3}$ dopants. It appears quite likely that the $\mathrm{Ce}^{4+}$ ions didn't create extra oxygen vacancies when replacing the $\mathrm{Zr}^{4+}$ ions; the stabilization being due mainly to the large ionic radius of $\mathrm{Ce}^{4+}$.

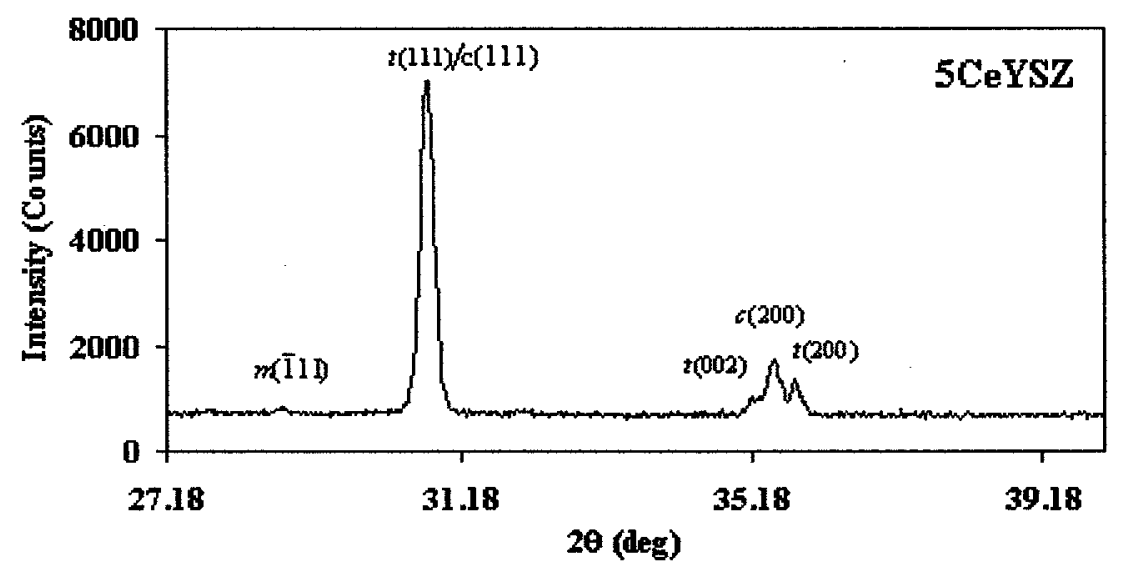

Figure 74 XRD patterns of the sintered 5CeYSZ sample.

In summary, Table 11 lists the phase structures and concentrations of each doped material. The relative lattice parameters for the cubic or tetragonal phase as well as the equivalent $\mathrm{YO}_{1.5}$ content in c-or t-phase were also calculated from the XRD patterns and given in the Table 11 .

It should be noted that the existence of small particles with higher dopant contents shown in the backscattered electron images in Figure 69 were not detected by XRD due to the small amounts and the limited the sensitivity of the equipment. 
Table 11 Phases present and phase concentrations obtained from XRD patterns

\begin{tabular}{|c|c|c|c|c|c|}
\hline Sample ID & $\begin{array}{c}m- \\
\text { phase } \\
(\mathrm{mol} \% \\
)\end{array}$ & $\begin{array}{c}c \text { and } t- \\
\text { phases } \\
(\mathrm{mol} \%)\end{array}$ & $\begin{array}{c}\text { Lattice constant } \\
\text { for c- phase } \\
(\mathrm{nm})\end{array}$ & $\begin{array}{c}\text { Axial ratio } \\
\text { c/a for } t- \\
\text { phase }\end{array}$ & $\begin{array}{c}\text { Equivalent } \mathrm{YO}_{1.5} \\
\text { content in c-or t- } \\
\text { phase (mol\%) }\end{array}$ \\
\hline 7YSZ & 45.96 & $55.04 c$ & 0.5140 & - & 17.66 \\
\hline 3.9TaYSZ & 100 & 0 & - & - & 0 \\
\hline $3.9 \mathrm{NbYSZ}$ & 96.52 & $3.48 t$ & - & - & 17.05 \\
\hline 5CrYSZ & 51.52 & $48.48 c$ & 0.5139 & - & 11.55 \\
\hline 5ScYSZ & 5.73 & $94.27 c$ & 0.5128 & - & 23.35 \\
\hline 5YbYSZ & - & $100 c$ & 0.5140 & - & 11.587 \\
\hline 5CeYSZ & 2.49 & $97.51 t+c$ & - & 1.006948 & \\
\hline
\end{tabular}

\subsection{Prediction of Phases from Reference Phase Diagrams}

To better analyze the EDS and XRD results, the predicted phases present and the relative content of each phase are described for each doped material from the reference $\mathrm{ZrO}_{2}$ $\mathrm{Y}_{2} \mathrm{O}_{3}$ phase diagram in this section. Three assumptions were made in determining phases present in the doped zirconia alloys:

1. All the samples were sintered at $1500^{\circ} \mathrm{C}$ for $120 \mathrm{hrs}$ and then cooled at a very slow cooling rate $\left(<5^{\circ} \mathrm{C} / \mathrm{min}\right)$, as shown in Figure 50. Under these conditions, the diffusion within the zirconia alloys was assumed to be complete and an equilibrium state was assumed to have been reached when cooling was complete.

2. The ternary phase information for the co-doped ziconia alloys is scarce. Since the phase stability and phase transformation of $\mathrm{ZrO}_{2}$ are primarily controlled by 162 
the content of oxygen vacancies, and the addition of metal oxides to $\mathrm{ZrO}_{2}$ may either create or annihilate oxygen vacancies, our phase analysis will be mainly based on the effect of oxygen vacancies on the $\mathrm{t}-$ or $\mathrm{c}-\mathrm{ZrO} 2$ stabilization. The equilibrium phase diagram for the $\mathrm{Y}_{2} \mathrm{O}_{3}-\mathrm{ZrO}_{2}$ binary system was adopted in the phase analysis for all the co-doped samples, where the concentration of oxygen vacancies in each system is considered. The calculated percentages of oxygen vacancy for each sample are given in Table 12.

3. In addition to the oxygen vacancies, the addition of a cation that has a larger ionic radius than $\mathrm{Zr}^{4+}$ has an effect on the stabilization of $\mathrm{t}-$ or $\mathrm{c}-\mathrm{ZrO}_{2}$ as well, although its degree of stabilization is much less than that of an oxygen vacancy. For example, in the $\mathrm{CeO}_{2}$ stabilized $\mathrm{ZrO}_{2}$ system, no oxygen vacancies are created since the valence of $\mathrm{Ce}^{4+}$ is the same as that of $\mathrm{Zr}^{4+}$. In such a system, the cubic or tetragonal $\mathrm{ZrO}_{2}$ is stabilized by the large $\mathrm{Ce}^{4+}$ ion although the effect of $20 \mathrm{~mol} \% \mathrm{CeO}_{2}$ stabilizing the c-phase in the $\mathrm{CeO}_{2}-\mathrm{ZrO}_{2}$ system is almost the same as that of $9 \mathrm{~mol} \% \mathrm{Y}_{2} \mathrm{O}_{3}$ in the system $\mathrm{Y}_{2} \mathrm{O}_{3}-\mathrm{ZrO}_{2}$ [22]. Thus, there will be some variability for each doped system due to the different dopant cations. These variations will be discussed qualitatively.

The phase diagram of the $\mathrm{Y}_{2} \mathrm{O}_{3}-\mathrm{ZrO}_{2}$ system is shown in Figure 75. From this phase diagram, it is expected that the zirconia alloys with an oxygen vacancy content between $1.7 \mathrm{~mol} \%$ to $6.9 \mathrm{~mol} \%$ will fall within the two-phase region at $1500^{\circ} \mathrm{C}$. Since $7 \mathrm{YSZ}$ has about $3.8 \mathrm{~mol} \%$ of oxygen vacancies, it lies in the two-phase region at position $\mathrm{A}$ in the phase diagram. Likewise, the number of oxygen vacancies for the $7 \mathrm{YSZ}-\mathrm{CeO}_{2}$ system is 
the same as for 7YSZ, and it situates at position $\mathrm{A}$ as well. The $5 \mathrm{Cr} 7 \mathrm{YSZ}, 5 \mathrm{ScYSZ}$, $5 \mathrm{YbYSZ}$ and $12 \mathrm{YSZ}$ systems with the concentration of about $5.97 \mathrm{~mol} \%$ oxygen vacancies are all located at $\mathrm{B}$ in the two-phase region. On the other hand, the 3.9TaYSZ and $3.9 \mathrm{NbYSZ}$ systems have only about $0.0055 \mathrm{~mol} \%$ oxygen vacancies due to their annihilation when the pentavalent cations were added to $\mathrm{ZrO}_{2}$. Thus these two-doped samples move into the single tetragonal phase region at position $\mathrm{C}$ at $1500^{\circ} \mathrm{C}$, close to the pure $\mathrm{ZrO}_{2}$.

Table 12 The calculated oxygen percentages for each sample

\begin{tabular}{|c|c|}
\hline Sample ID & Oxygen vacancies (mol\% per 100 cations) \\
\hline 7 YSZ & 3.8 \\
\hline $3.9 \mathrm{TaYSZ}$ & 0.0055 \\
\hline $3.9 \mathrm{NbYSZ}$ & 0.0055 \\
\hline $5 \mathrm{CrYSZ}$ & 5.97 \\
\hline $5 \mathrm{ScYSZ}$ & 5.97 \\
\hline $5 \mathrm{YbYSZ}$ & 5.97 \\
\hline $5 \mathrm{CeYSZ}$ & 3.615 \\
\hline $12 \mathrm{YSZ}$ & 5.97 \\
\hline
\end{tabular}

At points $\mathrm{A}$ and $\mathrm{B}$, the equilibrium phase consists of tetragonal phase with about $1.7 \mathrm{~mol} \%$ oxygen vacancies and cubic phase with about $6.9 \mathrm{~mol} \%$ oxygen vacancies. Using the lever rule, the fractions of the tetragonal and cubic phase for both 7YSZ and $5 \mathrm{CeYSZ}$ at point $\mathrm{A}$ were calculated to be about $59.62 \mathrm{~mol} \%$ and $40.38 \mathrm{~mol} \%$, respectively; and the fractions of the tetragonal and cubic phase for $5 \mathrm{CrYSZ}, 5 \mathrm{ScYSZ}$, 
and $5 \mathrm{YbYSZ}$ systems at point B were about $17.88 \mathrm{~mol} \%$ and $82.12 \mathrm{~mol} \%$, respectively. At point $\mathrm{C}$, samples comprised of $7 \mathrm{YSZ}-\mathrm{TaO}_{2.5}$ and $7 \mathrm{YSZ}-\mathrm{NbO}_{2.5}$ contained tetragonal single phase.

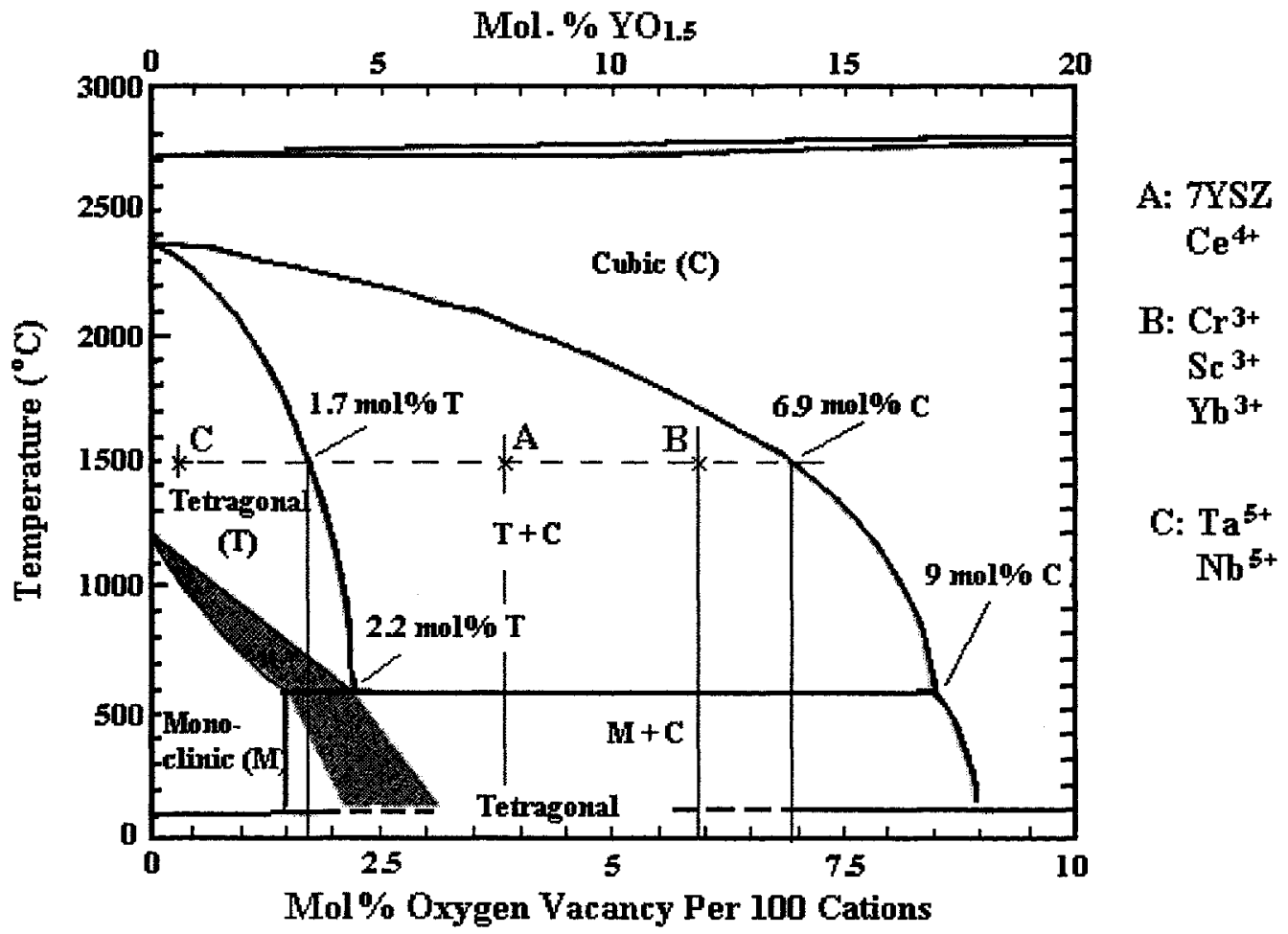

Figure 75 Phase diagram for the $\mathrm{Y}_{2} \mathrm{O}_{3}-\mathrm{ZrO}_{2}$ binary system. The positions of eight doped samples studied are labeled in the phase diagram [25].

When cooled down to room temperature at a very slow cooling rate, the phases in the A and $\mathrm{B}$ regions experience transformations from cubic to tetragonal and tetragonal to monoclinic. The tetragonal phase for samples with less than $1.5 \mathrm{~mol} \%$ oxygen vacancies will transform to monoclinic phase; and samples with greater than 9 mol\% oxygen vacancies will keep the cubic phase. The $c+t$ phases for the 7YSZ, 5CrYSZ, 5ScYSZ, 
5YbYSZ and 5CeYSZ samples will transform into $m+c$ phases upon cooling. The calculated phase fractions for 7YSZ and 5CeYSZ are about $69.33 \mathrm{~mol} \%$ monoclinic phase with $1.5 \mathrm{~mol} \%$ oxygen vacancies and $30.67 \mathrm{~mol} \%$ cubic phase with $9 \mathrm{~mol} \%$ oxygen vacancies respectively; and the phase fractions for $5 \mathrm{CrYSZ}, 5 \mathrm{ScYSZ}$, and $5 \mathrm{YbYSZ}$ samples are about $40.4 \mathrm{~mol} \%$ monoclinic phase with $1.5 \mathrm{~mol} \%$ oxygen vacancies and $59.6 \mathrm{~mol} \%$ cubic phase with $9 \mathrm{~mol} \%$ oxygen vacancies, respectively. For $3.9 \mathrm{TaYSZ}$ and 3.9 NbYSZ with oxygen vacancies less than $1.5 \mathrm{~mol} \%$, at room temperature, the equilibrium phase is $100 \mathrm{~mol} \%$ monoclinic. The calculated relative content of each phase based on the equilibrium $\mathrm{Y}_{2} \mathrm{O}_{3}-\mathrm{ZrO}_{2}$ binary system phase diagram at $1500^{\circ} \mathrm{C}$ and room temperature for all the samples are listed in Table 13.

Table 13 The relative content of each phase calculated for all the samples

\begin{tabular}{|c|c|c|c|c|}
\hline \multirow[b]{2}{*}{ Sample ID } & \multicolumn{2}{|c|}{$1500^{\circ} \mathrm{C}(\mathrm{c}+\mathrm{t})$} & \multicolumn{2}{|c|}{ Rom Temp. $(c+m)$} \\
\hline & $\begin{array}{c}\text { c-phase with } \\
6.9 \text { mol\% oxygen } \\
\text { vacancy } \\
(\mathrm{mol} \%)\end{array}$ & $\begin{array}{c}\text { t-phase with } \\
1.7 \text { mol\% oxygen } \\
\text { vacancy } \\
\text { (mol\%) }\end{array}$ & $\begin{array}{l}\text { c-phase with } \\
\text { 9mol\% oxygen } \\
\text { vacancy } \\
(\mathrm{mol} \%)\end{array}$ & $\begin{array}{c}\text { m-phase with } \\
1.5 \text { mol\% oxygen } \\
\text { vacancy } \\
\text { (mol\%) }\end{array}$ \\
\hline $7 Y S Z$ & 40.38 & 59.62 & 30.57 & 69.42 \\
\hline 3.9TaYSZ & 0 & 100 & 0 & 100 \\
\hline 3.9NbYSZ & 0 & 100 & 0 & 100 \\
\hline 5CrYSZ & 82.17 & 17.83 & 59.71 & 40.29 \\
\hline 5ScYSZ & 82.21 & 17.79 & 59.81 & 40.19 \\
\hline $5 \mathrm{YbYSZ}$ & 82.15 & 17.85 & 59.63 & 40.37 \\
\hline 5CeYSZ & 36.77 & 63.23 & 28.19 & 71.81 \\
\hline
\end{tabular}




\subsection{Estimation of Compositions of Dopants and Oxygen Vacancies}

Comparing the predictions from the phase content and the results from $\mathrm{XRD}$, it was found that some discrepancies existed. Two reasons are thought to be for this: one is the insufficient time for diffusion during cooling; another is the factor of ionic radius of the doped cations, which is not considered in the phase calculation. However, the effect of ionic radius of dopant cations on stabilization of the tetragonal or cubic $\mathrm{ZrO} 2$ is not negligible.

The calculation of the degree of stabilization for different doped cations by Kuwabara et al. [21] showed that the rare earth metal oxides, such as $\mathrm{Yb}^{3+}$, which had almost the same valence and ionic radius as $\mathrm{Y}^{3+}$, were expected to have the same stabilizing effect as $\mathrm{Y}^{3+}$. Therefore, the degree of stabilization for these rare earth metal oxides was close to 1 . On the other hand, the degree of stabilization for $\mathrm{Sc}^{3+}$ is 0.45 and as such $\mathrm{Sc}_{2} \mathrm{O}_{3}$ had a lower effect on stabilization than $\mathrm{Y}^{3+}$, although it created the same number of oxygen vacancies. When the ionic radius of the dopant, such as $\mathrm{Sc}^{3+}$ cation, was smaller than that of $\mathrm{Zr}^{4+}$, it led to a decrease of lattice parameter when added to $\mathrm{ZrO}_{2}$ material and therefore reduced the stabilizing effect. In contrast, the addition of larger ions, such as $\mathrm{Ce}^{4+}$, increased the lattice parameter and was shown to lower the degree of stabilization (0.38) compared to the cations discussed above. An argument for the stabilization of $\mathrm{ZrO}_{2}$ by $\mathrm{Ce}^{4+}$ was because the $\mathrm{Ce}^{4+}$ ions might partially be reduced to $\mathrm{Ce}^{3+}$, which was associated with the creation of oxygen vacancies for charge neutrality [124].

This explained why the 5YbYSZ had more cubic phase than 5ScYSZ; and why 5CeYSZ 
sample also had the tetragonal phase. However, it seems that the addition of $\mathrm{Cr}^{3+}$ cations to 7YSZ had a totally opposite effect on the stabilizing of $\mathrm{ZrO}_{2}$. Since $\mathrm{Cr}^{3+}$ cations had an ionic radius of $0.063 \mathrm{~nm}$, smaller than that of $\mathrm{Zr}^{4+}$ cations, the effect of destabilization caused by the decrease in lattice constant had undermined the effect of stabilization caused by oxygen vacancies.

In general, the dopants or oxygen vacancies did not distribute homogenously when there were two or more phases present and the content of oxygen vacancies varied with the crystal structures. For example, for the 7YSZ sample, the content of oxygen vacancies within the monoclinic phase was much less than that within the cubic phase, same as the yttrium content. From the phase diagram, the contents of oxygen vacancies at room temperature for the doped samples were estimated to be of $1.5 \mathrm{~mol} \%$ within the monoclinic phase and $9 \mathrm{~mol} \%$ within the cubic phase, as shown Table 13 . However, this prediction was based on the $\mathrm{ZrO}_{2}-\mathrm{Y}_{2} \mathrm{O}_{3}$ phase diagram without considering the effects of ionic radius of the dopant cations and the cooling conditions. The actual phase concentrations detected by XRD were not the same as the predictions. Therefore, the distributions of oxygen vacancies were also different from the predictions. Considering all these factors, the concentration of oxygen vacancies and the dopants will be recalculated based on the XRD and EDX elemental analysis results, as well as the phase diagram in this section. In addition, during the calculation, except for 3.9TaYSZ and 3.9NbYSZ samples, all the remaining samples including those containing the monoclinic phase were assumed to have a concentration of $1.5 \mathrm{~mol} \%$ oxygen vacancies within the relative monoclinic phase. 


\subsubsection{YSZ}

In the $7 \mathrm{YSZ}$ system, $7.6 \mathrm{~mol} \% \mathrm{YO}_{1.5}$ was added to pure $\mathrm{ZrO}_{2}$. At room temperature, about $46.0 \mathrm{~mol} \%$ monoclinic phase and $54.0 \mathrm{~mol} \%$ cubic phase in this sample were detected by the XRD results, which are similar to that predicted from the $\mathrm{ZrO}_{2}-\mathrm{Y}_{2} \mathrm{O}_{3}$ equilibrium phase diagram. Thus, it can be assumed that the $7.6 \mathrm{~mol} \% \mathrm{YO}_{1.5}$ will partition between these two phases reaching an estimated $3.0 \mathrm{~mol} \%$ in the monoclinic phase and $11.3 \mathrm{~mol} \%$ in the cubic phase. The oxygen vacancies created by $\mathrm{Y}^{3+}$ is therefore about $1.5 \mathrm{~mol} \%$ in the monoclinic phase and $5.65 \mathrm{~mol} \%$ in the cubic phase.

\subsection{TaYSZ}

In the 3.9TaYSZ system, $7.056 \mathrm{~mol}^{\circ} \mathrm{YO}_{1.5}$ and $7.045 \mathrm{~mol} \% \mathrm{TaO}_{2.5}$ were added to the pure $\mathrm{ZrO}_{2}$. At room temperature, almost $100 \mathrm{~mol} \%$ monoclinic phase was observed from the XRD results, which indicated a lower content of oxygen vacancies. Since the oxygen vacancies created by $\mathrm{Y}^{3+}$ could be annihilated by the addition of $\mathrm{Ta}^{5+}[86]$, thus, only about $0.0055 \mathrm{~mol} \%$ oxygen vacancies might be remained in the monoclinic phase for the dopants of $7.056 \mathrm{~mol} \% \mathrm{Y}$ and $7.045 \mathrm{~mol} \% \mathrm{Ta}$.

\subsection{NbYSZ}

Similar to the 3.9TaYSZ sample, in the $3.9 \mathrm{NbYSZ}$ sample, $7.056 \mathrm{~mol} \% \mathrm{YO}_{1.5}$ and $7.045 \mathrm{~mol} \% \mathrm{NbO}_{2.5}$ were added to the pure $\mathrm{ZrO}_{2}$. Thus, these two samples had the similar monoclinic structures except for a small amount of tetragonal phase was observed for the 3.9NbYSZ. For the same reason, about $0.0055 \mathrm{~mol} \%$ oxygen vacancies might exist in 
both the monoclinic and tetragonal phases.

\subsubsection{CrYSZ}

In the $5 \mathrm{CrYSZ}$ system, about $7.233 \mathrm{~mol} \% \mathrm{YO}_{1.5}$ was added along with $\mathrm{CO}_{1.5}$ to pure $\mathrm{ZrO}_{2}$. At room temperature, it was divided into an estimated $3.0 \mathrm{~mol} \%$ in the monoclinic phase (Assuming no Cr cations exist in the monoclinic phase) and $11.73 \mathrm{~mol} \%$ in the cubic phase. The oxygen vacancies created by yttria is therefore about $1.5 \mathrm{~mol} \%$ in the monoclinic phase and about $5.86 \mathrm{~mol} \%$ in the cubic phase. The EDX elemental analysis indicated that the content of $\mathrm{Cr}$ cations was only about $1.17 \mathrm{~mol} \%$, which is thought to create about $0.59 \mathrm{~mol} \%$ oxygen vacancies in the cubic phase.

\subsection{5. $5 S e Y S Z$}

In the $5 \mathrm{ScYSZ}$ system, $4.711 \mathrm{~mol} \% \mathrm{ScO}_{1.5}$ and $7.233 \mathrm{~mol} \% \mathrm{YO}_{1.5}$ were added to pure $\mathrm{ZrO}_{2}$. At room temperature, about $5.7 \mathrm{~mol} \%$ monoclinic phase and $94.3 \mathrm{~mol} \%$ cubic phase were detected by XRD. The EDX elemental analysis in this sample showed a homogeneous distribution of both $\mathrm{Y}$ and Sc cations within each phase and a ratio of $\mathrm{Y} / \mathrm{Sc}$ was about $7.233 / 4.711$. Based on these results, it is estimated that $1.183 \mathrm{~mol} \% \mathrm{Sc}$ and $1.817 \mathrm{~mol} \% \mathrm{Y}$ are in the monoclinic phase; and $4.92 \mathrm{~mol} \% \mathrm{Sc}$ and $7.56 \mathrm{~mol} \% \mathrm{Y}$ are in the cubic phase. The oxygen vacancies created by these two dopant cations are therefore about $1.5 \mathrm{~mol} \%$ in the monoclinic phase and $6.24 \mathrm{~mol} \%$ in the cubic phase.

\subsubsection{YbYSZ}

Same as the 5ScYSZ sample, $4.711 \mathrm{~mol}^{2} \mathrm{YbO}_{1.5}$ and $7.233 \mathrm{~mol} \% \mathrm{YO}_{1.5}$ were added to 170 
pure $\mathrm{ZrO}_{2}$. However, only cubic phase was observed in the XRD patterns. Thus, the single cubic phase is assumed at room temperature. The content of oxygen vacancies created by these two types of cations is estimated to be $5.97 \mathrm{~mol} \%$ and the dopant contents for $\mathrm{Y}$ and $\mathrm{Yb}$ are $7.233 \mathrm{~mol} \%$ and $4.711 \mathrm{~mol} \%$, respectively.

\subsubsection{CeYSZ}

The 5CeYSZ sample contained $7.233 \mathrm{~mol} \% \mathrm{YO}_{1.5}$ and $4.711 \mathrm{~mol} \% \mathrm{CeO}_{2}$ as dopants. In this sample, only the $\mathrm{Y}$ cations contributed to the formation of oxygen vacancies. Since Ce had the same valence as $\mathrm{Zr}$, the replacement of $\mathrm{Zr}$ by $\mathrm{Ce}$ would not create oxygen vacancy. At room temperature, about $2.5 \mathrm{~mol} \%$ monoclinic phase and $97.5 \mathrm{~mol} \%$ tetragonal/cubic phases were observed from XRD patterns of the $5 \mathrm{CeYSZ}$ sample. In addition, from the EDX elemental analysis results, about $5.88 \mathrm{~mol} \% \mathrm{Y}$ and $3.36 \mathrm{~mol} \% \mathrm{Ce}$ were observed to be within the shadowed grains and $14.85 \mathrm{~mol} \% \mathrm{Y}$ and $5.52 \mathrm{~mol} \% \mathrm{Ce}$ were within the bright grains. These data provided the evidence of the existence of both tetragonal and cubic phases. Combined with the XRD results for the relative content of each phase, the tetragonal phase was estimated to be $81.66 \mathrm{~mol} \%$ and the cubic phase was $15.84 \mathrm{~mol} \%$.

The $7.233 \mathrm{~mol} \% \mathrm{Y}$ therefore partitioned between these three phases, resulting in a content of about $3.0 \mathrm{~mol} \% \mathrm{Y}$ in the monoclinic phase, $5.88 \mathrm{~mol} \%$ in the tetragonal phase and $14.85 \mathrm{~mol} \%$ in the cubic phase. Considering the small amount of monoclinic phase, the Ce cations within the monoclinic phase were assumed to be zero. Thus, the $4.711 \mathrm{~mol} \%$ Ce only partitioned between the tetragonal phase with an estimated content of $3.36 \mathrm{~mol} \%$ 
and the cubic phase with an estimated content of $5.52 \mathrm{~mol} \%$. The contents of oxygen vacancies created by $\mathrm{Y}$ are therefore about $1.5 \mathrm{~mol} \%, 2.94 \mathrm{~mol} \%$ and $7.43 \mathrm{~mol} \%$ in the monoclinic, tetragonal and cubic phases, respectively.

Based on the discussion above, the contents of dopants and oxygen vacancies in each phase as well as the phase fraction for each sample were estimated and the data are listed in Table 14.

Table 14 The contents of dopants and oxygen vacancies within each phase and phase fractions

\begin{tabular}{|c|c|c|c|c|c|c|c|c|c|c|c|c|}
\hline \multirow{2}{*}{ Sample ID } & \multicolumn{3}{|c|}{$\begin{array}{c}\text { m-phase } \\
(\mathrm{mol} \%)\end{array}$} & \multicolumn{3}{c|}{$\begin{array}{c}\text { t-phase } \\
(\mathrm{mol} \%)\end{array}$} & \multicolumn{3}{c|}{$\begin{array}{c}\text { c-phase } \\
\text { (mol\%) }\end{array}$} & \multicolumn{3}{c|}{$\begin{array}{c}\text { Phase fraction } \\
\text { (mol\%) }\end{array}$} \\
\cline { 2 - 13 } & $x_{v}$ & $x_{Y}$ & $x_{M}$ & $x_{v}$ & $x_{Y}$ & $x_{M}$ & $x_{v}$ & $x_{Y}$ & $x_{M}$ & $C_{m}$ & $C_{i}$ & $C_{c}$ \\
\hline 7YSZ & 1.50 & 3.00 & - & - & - & - & 5.65 & 11.30 & - & 46.00 & - & 54.00 \\
\hline 3.9 TaYSZ & 0.005 & 7.056 & 7.045 & - & - & - & - & - & - & 100 & - & - \\
\hline 3.9 NbYSZ & 0.005 & 7.056 & 7.045 & 0.005 & 7.056 & 7.045 & - & - & - & 96.52 & 3.48 & - \\
\hline $5 \mathrm{CrYSZ}$ & 1.50 & 3.00 & 0.00 & - & - & - & 6.45 & 11.73 & 1.17 & 51.52 & - & 48.48 \\
\hline 5ScYSZ & 1.50 & 1.82 & 1.18 & - & - & - & 6.24 & 7.56 & 4.92 & 5.73 & - & 94.3 \\
\hline 5YbYSZ & - & - & - & - & - & - & 5.97 & 7.23 & 4.71 & - & - & 100 \\
\hline 5CeYSZ & 1.50 & 3.00 & 0.00 & 2.94 & 5.88 & 3.36 & 7.43 & 14.85 & 5.52 & 2.49 & 81.66 & 15.84 \\
\hline
\end{tabular}

Note: $x_{v}, x_{Y}$, and $x_{M}$ denoted the contents of oxygen vacancy; $\mathrm{Y}$ cation, and dopant cation, respectively; where $\mathrm{M}=\mathrm{Ta}, \mathrm{Nb}, \mathrm{Cr}, \mathrm{Sc}, \mathrm{Yb}$, and Ce. $C_{m}, C_{t}$, and $C_{c}$ denoted the contents of monoclinic, tetragonal and cubic phases, respectively. 


\subsection{Effects of Dopants on Phase Transformations and Specific Heats}

To obtain the information on phase stability and phase transition at high temperatures as well as the values of specific heat of the co-doped materials, the DSC analysis was also conducted. The results will be discussed as follows.

\subsubsection{DSC Analysis}

The DSC curves of each co-doped materials upon heating and cooling are shown in Figure 76, Figure 77, and Figure 78. From the DSC curves, upon heating at a rate of $20^{\circ} \mathrm{C} / \mathrm{min}$, it could be seen that all the materials exhibited both broadband endothermic (heat is taken in) and exothermic (heat is given out) regions, and the endothermic peaks were within the temperature range from 482 to $761^{\circ} \mathrm{C}$, and then the exothermic process happened. Whenever the endothermic or exothermic process occurred, the relative specific heat also changed. The transition temperatures upon heating for each doped material are summarized and listed in Table 15.

Table 15 Summary of phase transformation temperatures upon DSC heating

\begin{tabular}{|c|c|c|c|}
\hline Sample ID & $\mathrm{T}_{0}\left({ }^{\circ} \mathrm{C}\right)$ & $\mathrm{T}_{1}\left({ }^{\circ} \mathrm{C}\right)$ & $\mathrm{T}_{2}\left({ }^{\circ} \mathrm{C}\right)$ \\
\hline 7 YSZ & 562 & 661 & 761 \\
\hline $3.9 \mathrm{TaYSZ}$ & 582 & 642 & 761 \\
\hline $3.9 \mathrm{NbYSZ}$ & 482 & 502 & 761 \\
\hline $5 \mathrm{CrYSZ}$ & 533 & 661 & 761 \\
\hline $5 \mathrm{ScYSZ}$ & - & - & 761 \\
\hline $5 \mathrm{YbYSZ}$ & - & - & 761 \\
\hline $5 \mathrm{CeYSZ}$ & - & - & 761 \\
\hline
\end{tabular}

As seen from these curves, the co-doped materials can be classified into three different 173 
categories: (a) 5CrYSZ and 7YSZ; (b) $3.9 \mathrm{TaYSZ}$ and $3.9 \mathrm{NbYSZ}$; and (c) $5 \mathrm{ScYSS}$, $5 \mathrm{YbYSZ}$ as well as 5CeYSZ. This classification is consistent with the results obtained from XRD analysis, that is, the materials classified in the same group have similar phase concentrations. In the following section, these DSC curves will be discussed based on these three groups of materials.

\subsubsection{7YSZ and 5CrYSZ}

From XRD results, it is known that the 7YSZ and 5CrYSZ samples have very similar crystal structures and the relative content of each phase in spite of the $5 \mathrm{~mol}^{\circ} \mathrm{CrO}_{1.5}$ dopant into 7YSZ. That is, both $7 \mathrm{YSZ}$ and $5 \mathrm{CrYSZ}$ have almost $50 \mathrm{~mol} \%$ fraction of monoclinic structures. Similarly, the curves of these two materials also exhibit very similar phase transformation processes, as shown in Figure 76.

Upon heating, the two DSC curves both show an endothermic peak at a temperature of $661^{\circ} \mathrm{C}\left(\mathrm{T}_{1}\right)$. The endothermic peak at $661^{\circ} \mathrm{C}$ might involve the phase transition from monoclinic to tetragonal with $4 \mathrm{~mol} \% \mathrm{YO}_{1.5}$, which resulted in a change of specific heat. The onset temperatures are slightly different, for $7 \mathrm{YSZ}$, it is $562^{\circ} \mathrm{C}\left(\mathrm{T}_{0}\right)$, and for $5 \mathrm{CrYSZ}$, it is $533^{\circ} \mathrm{C}\left(\mathrm{T}_{0}\right)$. This difference may be caused by the $\mathrm{Cr}^{3+}$ dopant cation. With a further increase in temperature, the concentration of cubic phase increased due to the transformation from tetragonal to cubic, which might involve an exothermic process. At a temperature of $761^{\circ} \mathrm{C}\left(\mathrm{T}_{2}\right)$, the exothermic process is dominant for both materials, the DSC curves exhibit the exothermic process. Upon cooling, there is no apparent phase transition except a slight change in specific heat at $1081^{\circ} \mathrm{C}$, which may be caused by the 
phase transition. This can also be explained by transformation kinetics, that is, there is not enough time for the phase transition to occur due to the fast cooling rate $\left(20^{\circ} \mathrm{C} / \mathrm{min}\right)$.
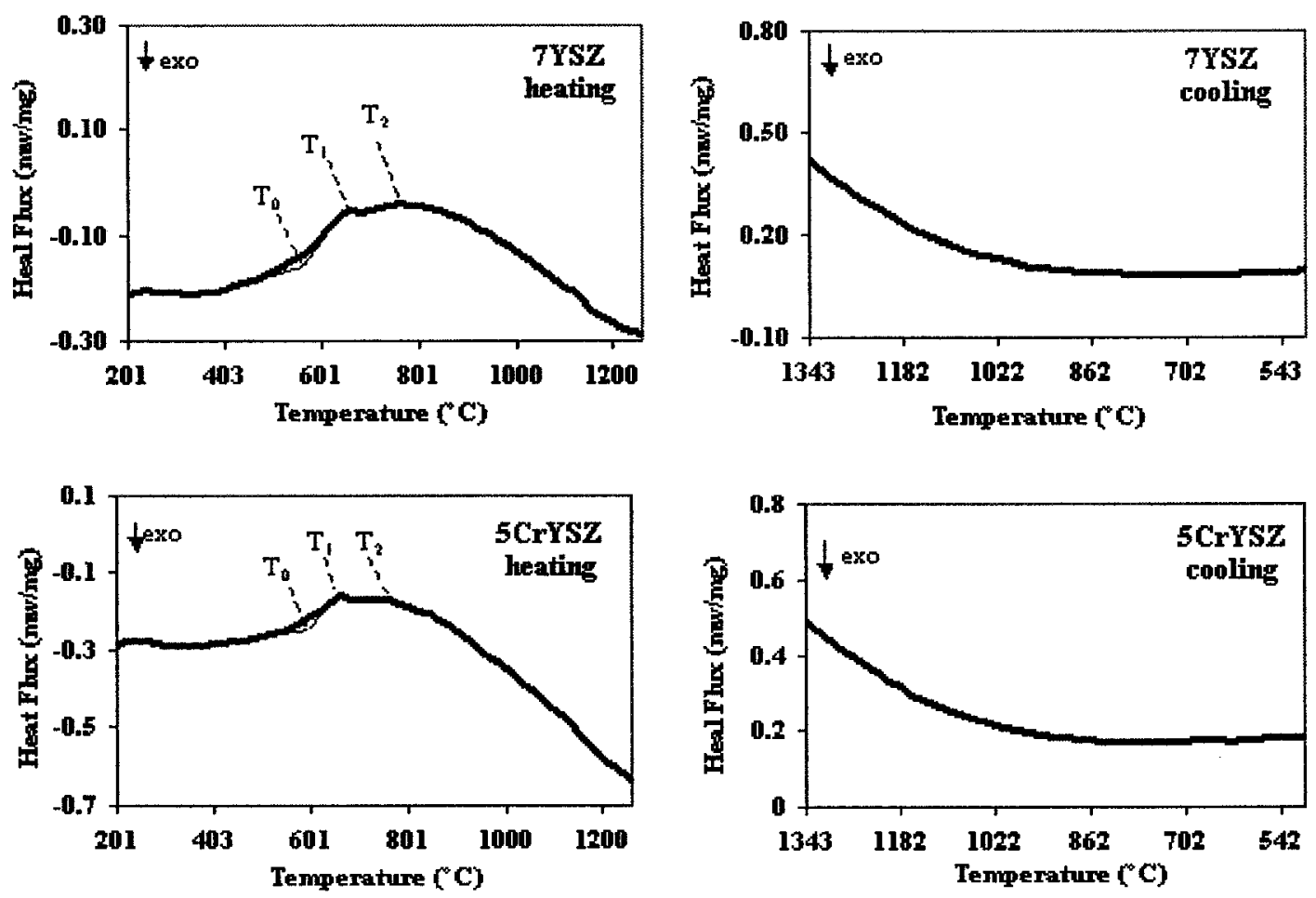

Figure 76 DSC curves for doped 7YSZ sintered materials upon heating and cooling at a rate of $20^{\circ} \mathrm{C} / \mathrm{min}$. (a) $7 \mathrm{YSZ}$; (b) $5 \mathrm{CrYSZ}$.

\subsubsection{3.9MYSZ $(\mathrm{M}=\mathrm{Ta}$ and $\mathrm{Nb})$}

The curves of both DSC of the 3.9TaYSZ and 3.9NbYSZ samples are shown in Figure 77. The DSC curve upon heating for 3.9TaYSZ exhibits one endothermic peak at a temperature of $642^{\circ} \mathrm{C}\left(\mathrm{T}_{1}\right)$. It indicates the transformation completion of from monoclinic to tetragonal phase. The intersection between two tangent lines gives the onset temperature of $582^{\circ} \mathrm{C}\left(\mathrm{T}_{0}\right)$, at which the phase transition from monoclinic to tetragonal begins and the specific heat starts to change. With further increase in 
temperature, a slight exothermic trend may be a result of the decomposition of tetragonal phase to two tetragonal structures with different dopant concentrations. In contrast, for 3.9NbYSZ, the endothermic peak for the transformation from monoclinic to tetragonal phase is at the temperature of $502^{\circ} \mathrm{C}$, and this transformation started at the temperature of $482^{\circ} \mathrm{C}$. As in the case of $3.9 \mathrm{TaYSZ}$, when temperature was higher than $761^{\circ} \mathrm{C}$, an exothermic process started. Between $502^{\circ} \mathrm{C}$ and $761^{\circ} \mathrm{C}$, a small endothermic peak was observed at $562^{\circ} \mathrm{C}$, which may be caused by the remaining tetragonal phase. Upon cooling, there are no exothermic phenomena observed for both samples.
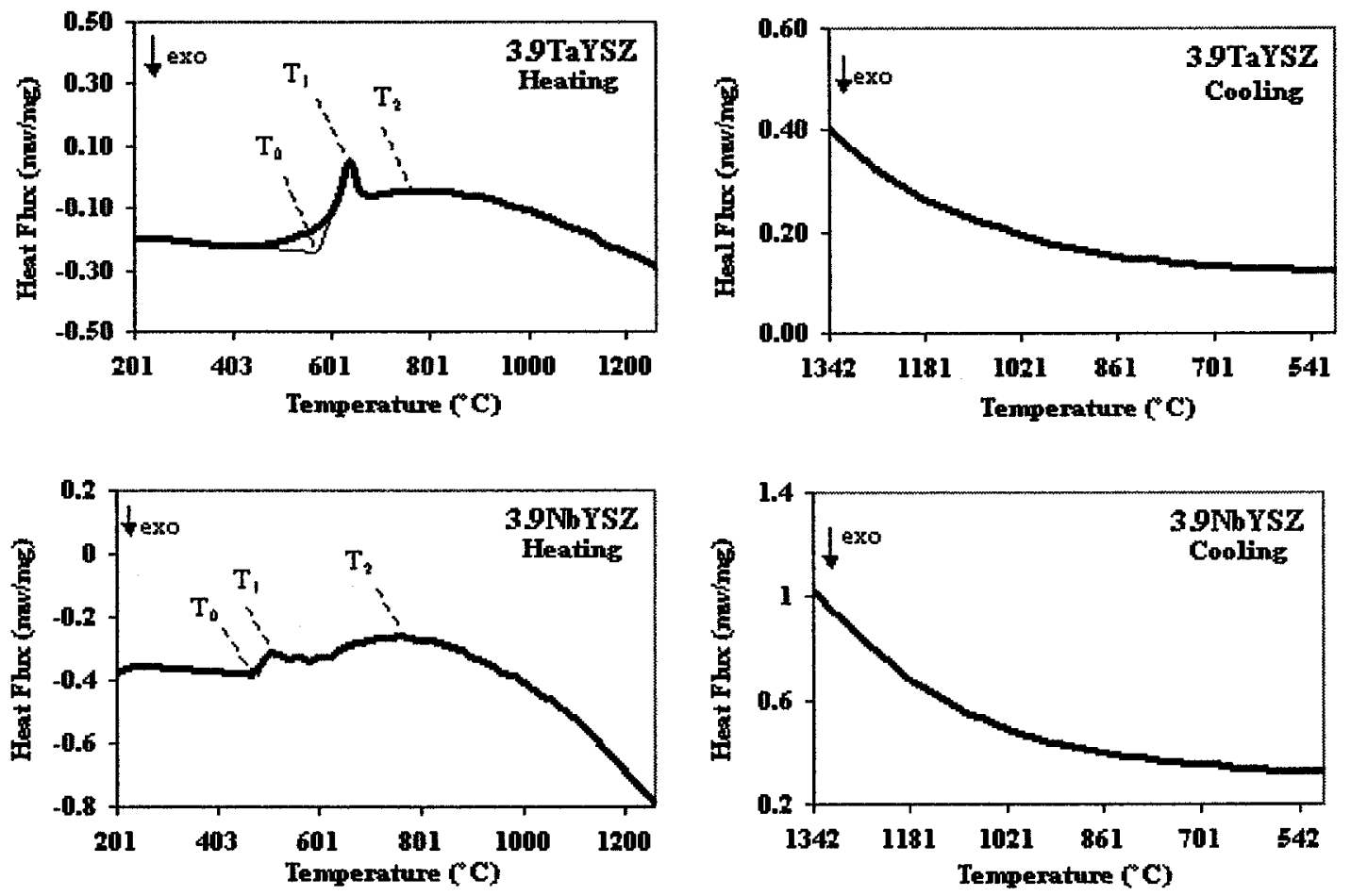

Figure 77 DSC curves for doped 7YSZ sintered materials upon heating and cooling at a rate of $20^{\circ} \mathrm{C} / \mathrm{min}$. (a) $3.9 \mathrm{TaYSZ}$; (b) $3.9 \mathrm{NbYSZ}$.

5.9.1.3. 5MYSZ $(M=S c, Y b$, and $C e)$ 
The DSC curves of the 5MYSZ $(\mathrm{M}=\mathrm{Sc}, \mathrm{Yb}$, and $\mathrm{Ce})$ samples are shown in Figure 78.
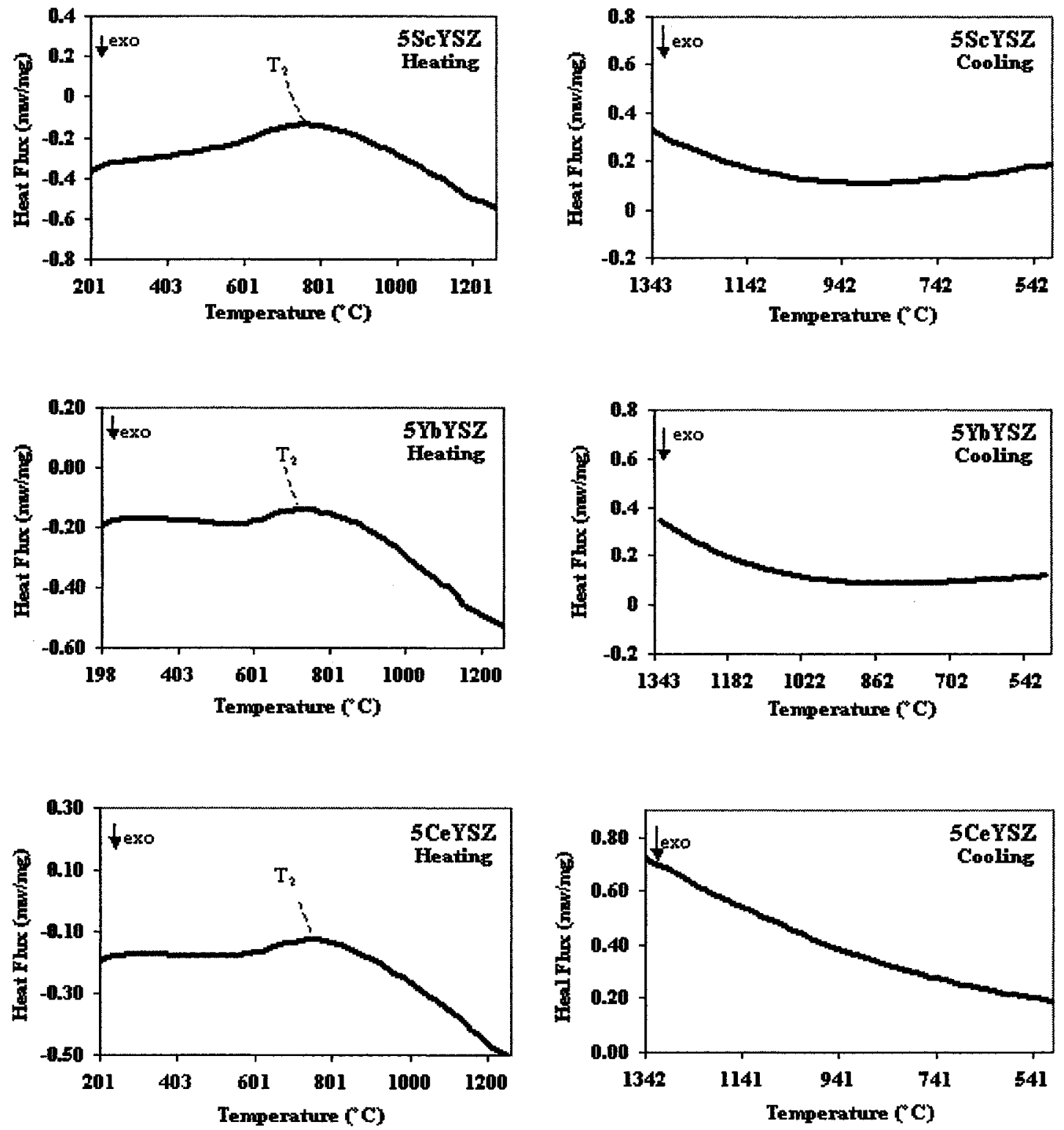

Figure 78 DSC curves for doped 7YSZ sintered materials upon heating and cooling at a rate of $20^{\circ} \mathrm{C} / \mathrm{min}$. (a) $5 \mathrm{ScYSZ}$; (b) $5 \mathrm{YbNbYSZ}$; and (c) $5 \mathrm{CeYSZ}$.

There are no apparent endothermic or exothermic peaks observed from DSC curves for 177 
5ScYSZ, 5YbYSZ and 5CeYSZ samples, as shown in Figure 78. Only at the temperature of $761^{\circ} \mathrm{C}$, the specific heat started to change and exhibit a slow exothermic process. This is because the endothermic transition might be caused by the phase transition from monoclinic to tetragonal. However, for the 5ScYSZ, 5YbYSZ and 5CeYSZ samples, only a small amount of monoclinic or no monoclinic phase was observed. For the slow exothermic process, it may be caused by the diffusion from high content of oxygen vacancies $(9 \mathrm{~mol} \%)$ to small content oxygen vacancies $(6.9 \mathrm{~mol} \%)$. Upon cooling, a change of specific heat occurred at $900^{\circ} \mathrm{C}$ for $5 \mathrm{ScYSZ}$ and $1101^{\circ} \mathrm{C}$ for $5 \mathrm{YbYSZ}$. As for 5CeYSZ, no specific heat change was found.

\subsubsection{Specific Heat of Each Doped Material}

The specific heat of each doped material was calculated using (Eq. 67) and the DSC results obtained from three measurements for the baseline, a standard sapphire and the corresponding sample. Similar to the DSC curves, the specific heats of the doped materials can also be classified into three categories: (a) 5CrYSZ and 7YSZ; (b) 3.9TaYSZ and 3.9NbYSZ; and (c) 5ScYSZ, 5YbYSZ and 5CeYSZ. The variations of specific heat with temperature of these doped materials upon heating are shown in Figure 79. The appearing of the specific heat peaks shown in Figure 79 (a) and (b) resulted from the transition from monoclinic to tetragonal phase. In contrast, no specific heat peaks were observed from Figure 79 (c) due to the lack of monoclinic phase. Specific heat data are required for the calculation of thermal conductivities, and the values are given in Table 16 for each sample at $100^{\circ} \mathrm{C}, 600^{\circ} \mathrm{C}$ and $800^{\circ} \mathrm{C}$. 

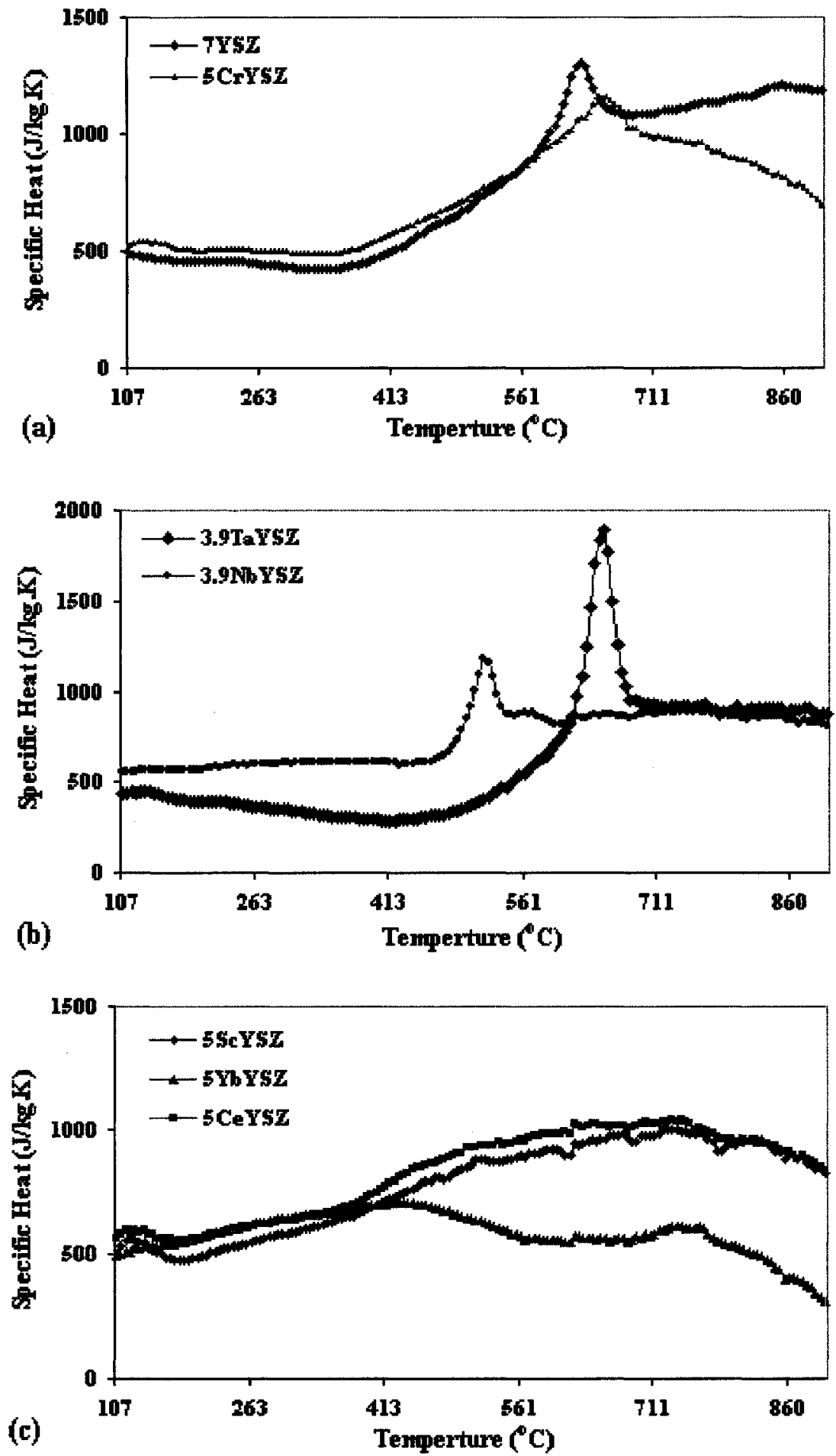

Figure 79 Specific heat of the doped samples (a) 7YSZ and 5CrYSZ; (b) 3.9TaYSZ and 3.9NbYSZ; and (c) 5ScYSZ, $5 \mathrm{YbYSZ}$ and 5CeYSZ. 
Table 16 The values of measured specific heat of doped materials using DSC

\begin{tabular}{|c|c|c|c|}
\hline \multirow{2}{*}{ Sample ID } & \multicolumn{3}{|c|}{ Specific heat Cp (J/Kg·K) } \\
\hline & $100^{\circ} \mathrm{C}$ & $600^{\circ} \mathrm{C}$ & $800^{\circ} \mathrm{C}$ \\
\hline $7 Y S Z$ & 491.70 & 1076.31 & 1153.54 \\
\hline 3.9TaYSZ & 438.52 & 739.56 & 920.38 \\
\hline 3.9NbYSZ & 554.55 & 819.71 & 864.48 \\
\hline 5CrYSZ & 525.40 & 975.46 & 896.04 \\
\hline $5 \mathrm{ScYSZ}$ & 496.51 & 918.26 & 940.45 \\
\hline $5 Y b Y S Z$ & 490.14 & 557.58 & 533.51 \\
\hline $5 \mathrm{CeYSZ}$ & 565.39 & 984.91 & 948.75 \\
\hline
\end{tabular}

Table 17 The values of specific heat of doped materials from publications

\begin{tabular}{|c|c|c|c|}
\hline \multirow{2}{*}{ Sample ID } & \multicolumn{3}{|c|}{ Specific heat $\mathrm{Cp}(\mathrm{J} / \mathrm{Kg} \cdot \mathrm{K})$} \\
\hline & $22^{\circ} \mathrm{C}$ & $600^{\circ} \mathrm{C}$ & $800^{\circ} \mathrm{C}$ \\
\hline 7YSZ [125] & 490.04 & 628.88 & 653.38 \\
\hline 3.9TaYSZ[125] & 432.83 & 571.96 & 579.69 \\
\hline 3.9NbYSZ[125] & 438.38 & 600.75 & 608.87 \\
\hline 5CrYSZ & - & - & - \\
\hline 5ScYSZ [126] & 500.33 & 642.09 & 667.11 \\
\hline 5YbYSZ [125] & 516.06 & 666.9 & 698.66 \\
\hline $5 \mathrm{CeYSZ}[75]$ & 470.54 & - & - \\
\hline
\end{tabular}

For comparison, some data on specific heat obtained from publications are listed in Table 17. From these values, it can be seen that at low temperatures, the specific heat for all the doped materials are very similar. The measured data at $100^{\circ} \mathrm{C}$ was slightly higher than the published data at room temperature except for the 5ScYSZ and 5YbYSZ. At high 180 
temperatures, the measured specific heat values, especially for the $7 \mathrm{YSZ}, 5 \mathrm{CrYSZ}$ 3.9TaYSZ, and 3.9NbYSZ samples, were higher than the corresponding published values. This is because the specific heat measured by Leclercq and Meverl [125] was based on single-phase samples without phase transformation at high temperatures; whereas the samples measured in our study experienced phase transformations at high temperatures and therefore resulted in high deviations in specific heat values. In addition, the loss of oxygen at high temperatures due to the inert gas environment during DSC running also caused some variability.

\subsection{Effects of Dopants on Materials Elastic Modulus}

The elastic modulus of each sample was measured using a nano-indentation tester. A force of $50 \mathrm{mN}$ was applied. The curves of load versus displacement of $5 \mathrm{YbYSZ}$ and 5CeYSZ are shown in Figure 80.
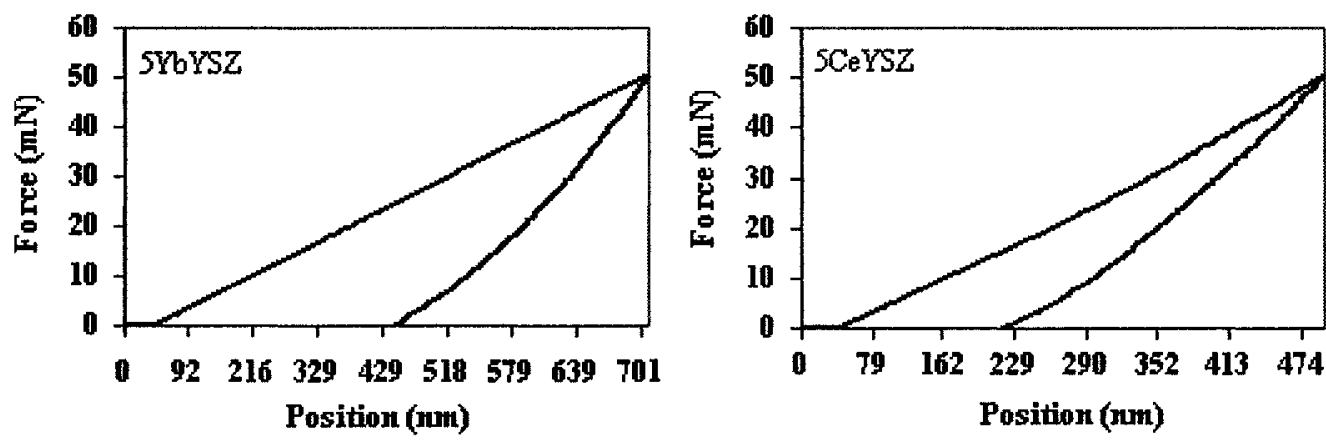

Figure 80 The load-displacement curves of $5 \mathrm{YbYSZ}$ and $5 \mathrm{CeYSZ}$ samples.

To better illustrate how the dopants affect the material's elastic modulii, the elastic modulus of each sample with zero porosity was evaluated using Mackenzie's equation (Eq. 71) [121]. The volume fractions $P$ of porosity were obtained from back scattering 
images using an image analyzer. The values of elastic modulus in samples containing porosity $(E)$, along with corresponding hardness values and the elastic modulus in samples without porosity $\left(E_{0}\right)$ are listed in Table 18. Comparing the data shown in Table 18 , it can be seen that the elastic moduli of the 7YSZ, 3.9TaYSZ, and 5CrYSZ materials are lower than those of the other doped materials. The $5 \mathrm{CrYSZ}$ has the lowest value and the $5 \mathrm{ScYSZ}$ has the highest value. However, as a TBC material, low value of elastic modulus can be beneficial for releasing the stress caused by the mismatch of thermal expansion coefficients between ceramic topcoat and metal substrate.

Table 18 Values of elastic modulus and hardness of each doped material at $50 \mathrm{mN}$ loads

\begin{tabular}{|c|c|c|c|c|}
\hline \multirow{2}{*}{ Sample ID } & $\begin{array}{c}\text { Porosity } P \\
(\text { vol.\%) }\end{array}$ & \multicolumn{2}{|c|}{ Elastic modulus (GPa) } & \multirow{2}{*}{ Hardness (MPa) } \\
\cline { 3 - 4 } & 13.19 & 106.65 & 139.40 & 12727 \\
\hline 7YSZ & 8.81 & 103.43 & 123.19 & 10470 \\
\hline 3.9 TaYSZ & 4.68 & 168.82 & 184.90 & 9306 \\
\hline 3.9 NbYSZ & 12.13 & 46.568 & 59.49 & 6679 \\
\hline 5CrYSZ & 13.36 & 191.04 & 250.63 & 17994 \\
\hline 5ScYSZ & 14.12 & 116.36 & 155.42 & 16224 \\
\hline 5YbYSZ & 5.30 & 145.88 & 161.76 & 19433 \\
\hline 5CeYSZ & & & & $\mathrm{E}_{0}$ \\
\hline
\end{tabular}

Two factors contribute the magnitude of the elastic modulus: the bonding strength for the dense materials and the microstructures of the doped materials. The bonding strength could be affected by the atomic bonding and crystal structures. For example, the low elastic modulus for $\mathrm{Cr}^{3+}$ doped materials may be caused either by the weak bonding when the host $\mathrm{Zr}^{4+}$ cation is substituted by $\mathrm{Cr}^{3+}$ cations, or the presence of monoclinic phase which results in the microscracks inside the sintered samples. The microstructures with 182 
inherent cracks or porosity due to poor sintering may also contribute to the low modulus of elasticity. On the other hand, the high elastic modulus of the 5ScYSZ sample may be caused by its strong atomic bonding strength and its cubic crystal structure.

\subsection{Effects of Dopants on Thermal Diffusivity}

Thermal diffusivities of each doped material at room temperature, $600^{\circ} \mathrm{C}$ and $800^{\circ} \mathrm{C}$ were measured using the laser flash method and the values as well as their standard deviations are given in Table 19.

Table 19 Thermal diffusivity ( $\alpha$ ) and its standard deviation of each doped material at different temperature

\begin{tabular}{|c|c|c|c|}
\hline \multirow{2}{*}{ Sample ID } & $22^{\circ} \mathrm{C}$ & $600^{\circ} \mathrm{C}$ & $800^{\circ} \mathrm{C}$ \\
\cline { 2 - 4 } & $\alpha\left(10^{-7} \cdot \mathrm{m}^{2} / \mathrm{s}\right)$ & $\alpha\left(10^{-7} \cdot \mathrm{m}^{2} / \mathrm{s}\right)$ & $\alpha\left(10^{-7} \cdot \mathrm{m}^{2} / \mathrm{s}\right)$ \\
\hline $7 \mathrm{YSZ}$ & $12.00 \pm 0.14$ & $5.45 \pm 0.01$ & $5.78 \pm 0.01$ \\
\hline $3.9 \mathrm{TaYSZ}$ & $8.37 \pm 0.05$ & $1.52 \pm 0.01$ & $1.10 \pm 0.03$ \\
\hline $3.9 \mathrm{NbYSZ}$ & $9.29 \pm 0.01$ & $1.71 \pm 0.01$ & $1.49 \pm 0.01$ \\
\hline $5 \mathrm{CrYSZ}$ & $7.93 \pm 0.03$ & $5.27 \pm 0.02$ & $5.75 \pm 0.01$ \\
\hline $5 \mathrm{ScYSZ}$ & $7.60 \pm 0.07$ & $6.28 \pm 0.10$ & $5.98 \pm 0.05$ \\
\hline $5 \mathrm{YbYSZ}$ & $6.53 \pm 0.09$ & $5.18 \pm 0.02$ & $4.96 \pm 0.02$ \\
\hline $5 \mathrm{CeYSZ}$ & $9.65 \pm 0.04$ & $6.81 \pm 0.09$ & $6.47 \pm 0.02$ \\
\hline
\end{tabular}

Three measurements had been made for each sample. All the data fell within $14 \%$ of the best-fit line. This scatter may be caused by some uncertainty in the measurements due to 
inhomogeneous heating because of the small size of the incident beam $(8 \mathrm{~mm})$ and inconsistent sample diameters $(8 \sim 10 \mathrm{~mm})$. Heat may flow to the radial direction in a sample, which could also affect the temperature measurements.

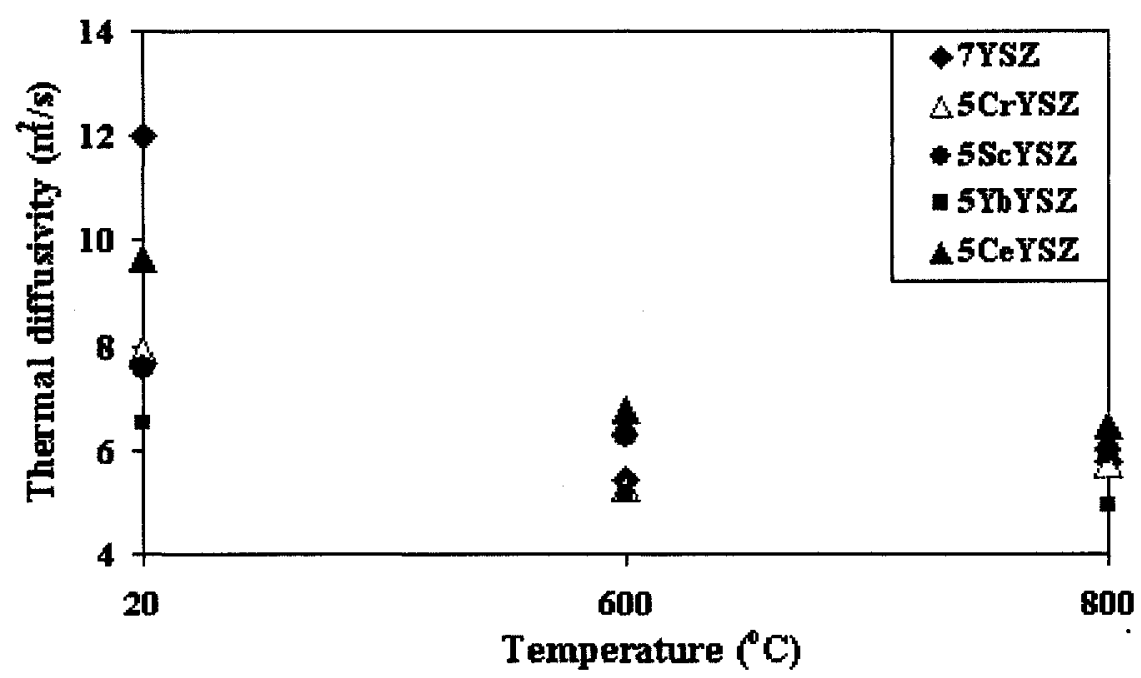

Figure 81 Thermal diffusivities of $\mathrm{MO}_{1.5}(\mathrm{M}=\mathrm{Cr}, \mathrm{Sc}$, and $\mathrm{Yb})$ and $\mathrm{CeO}_{2}$ doped $7 \mathrm{YSZ}$ as a function of temperature.

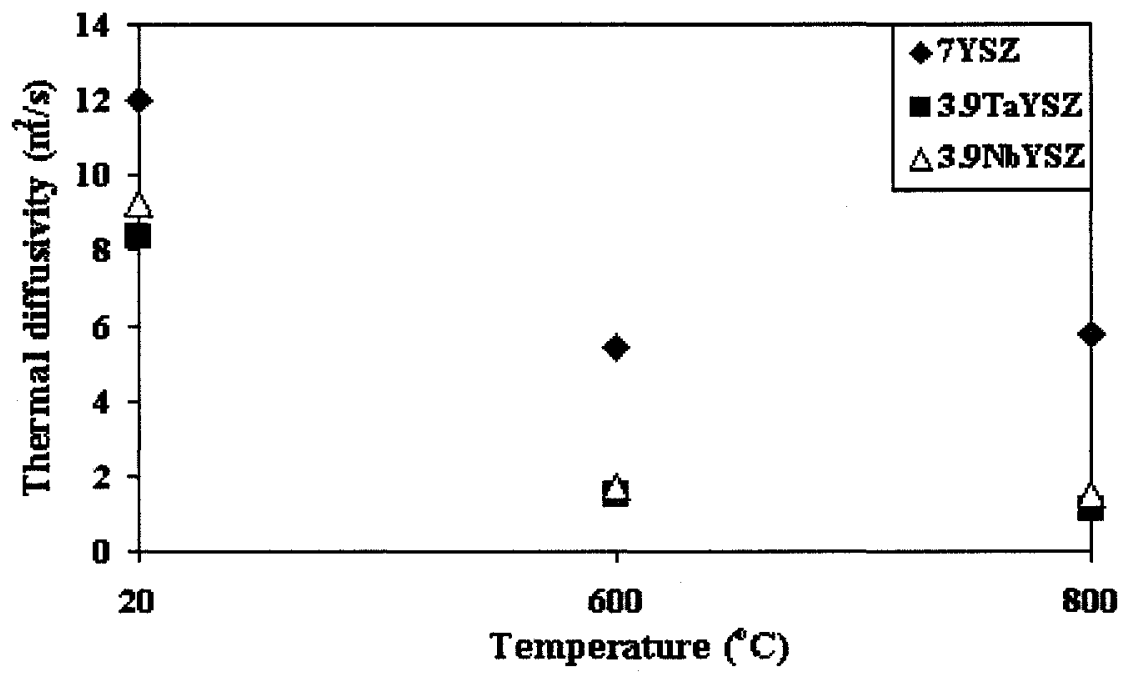

Figure 82 Thermal diffusivity of $\mathrm{MO}_{2.5}(\mathrm{M}=\mathrm{Ta}, \mathrm{Nb})$ doped $7 \mathrm{YSZ}$ as a function of temperature. 
The variations of the thermal diffusivities of the doped samples with temperature are shown in Figure 81 and Figure 82, which showed that the thermal diffusivities decreased with increase in temperature. This is consistent with the literature data. However, the data for 3.9TaYSZ and 3.9NbYSZ exhibited large scatter at temperatures of $600^{\circ} \mathrm{C}$ and $800^{\circ} \mathrm{C}$ from the trend line. The extremely low values at high temperatures resulted from the loose, powder-like structures due to phase transformation from tetragonal to monoclinic. When the measured temperature was lowered from $800^{\circ} \mathrm{C}$ to room temperature, both $3.9 \mathrm{TaYSZ}$ and $3.9 \mathrm{NbYSZ}$ specimens were found to have crumbled to powdery states.

\subsection{Calculation of Thermal Conductivity}

Thermal conductivities of the doped materials were calculated from the relationships of $\lambda=\rho \cdot \alpha \cdot C_{p}$, see (Eq. 72). The values of the density ( $\left.\rho\right)$, specific heat (Cp), and thermal diffusivity $(\alpha)$, are given in Table 9, Table 16, and Table 19. The thermal conductivities calculated from these measured parameters for sintered materials were lower than those of the dense samples due to the presence of porosity. Consequently, a correction factor needs to be introduced. Assume all the pores in the sintered samples are spherical; the thermal conductivities of the dense, porosity free materials can be evaluated using the equation [127]

$$
\frac{\lambda_{\text {porous }}}{\lambda_{\text {dense }}}=1-\frac{4 P}{3}
$$

where $\mathrm{P}$ is volume fraction of total porosity inside the sintered sample, which is obtained 
form image analysis and density measurement. The average values for each sample are shown in Table $10 ; \lambda_{\text {porous }}$ is the measured thermal conductivity of the material containing porosity; and $\lambda_{\text {dense }}$ is the corrected thermal conductivity with zero porosity in the sintered material.

Substituting the values of density, specific heat, thermal diffusivity and the fraction of porosity in the sintered samples into (Eq. 72) and (Eq. 78), the variations of thermal conductivity with temperature for all the sintered samples were obtained and are shown in Figure 83 and Figure 84, respectively. It should be noted that the values of specific heat measured at $100^{\circ} \mathrm{C}$ were used to calculate the thermal conductivities, which may have resulted in a certain systematic error due to the lack of data at room temperature.

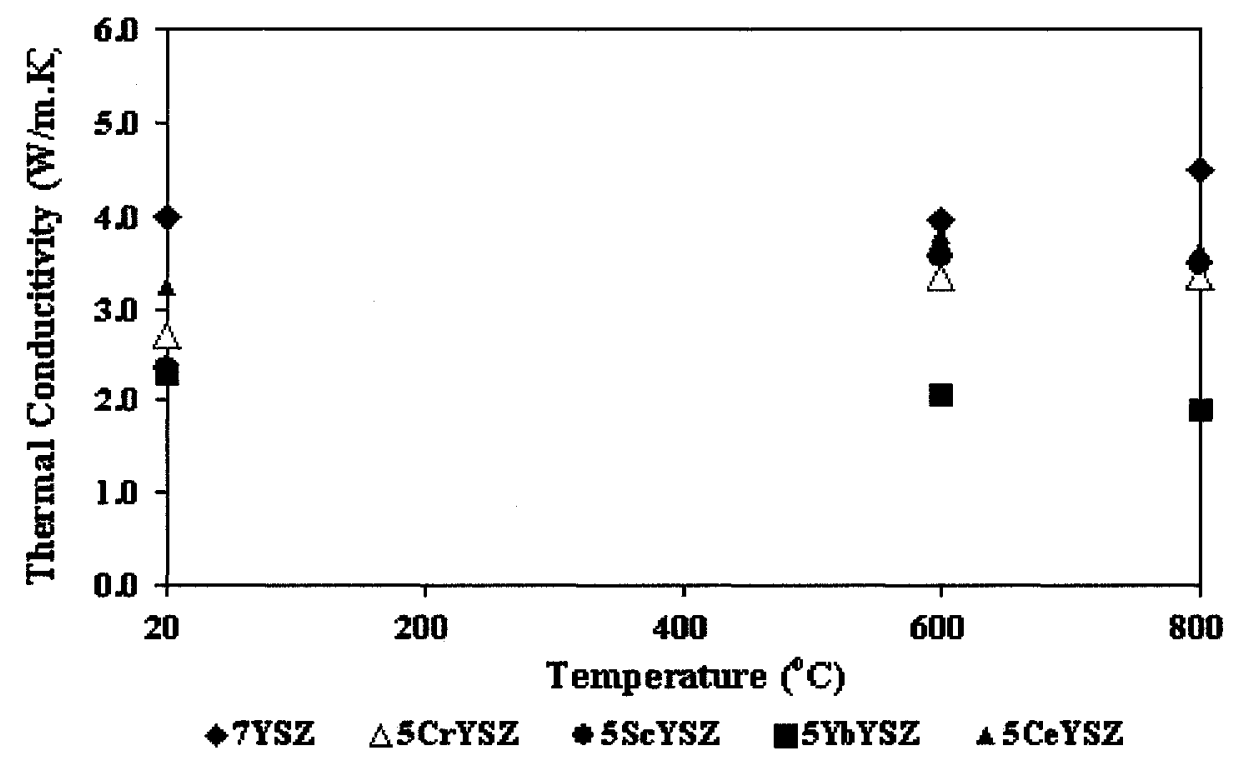

Figure 83 Variations of thermal conductivity as a function of temperatures for 7YSZ and 5MYSZ $(\mathrm{M}=\mathrm{Cr}, \mathrm{Sc}, \mathrm{Yb}$, and $\mathrm{Ce})$ samples. 


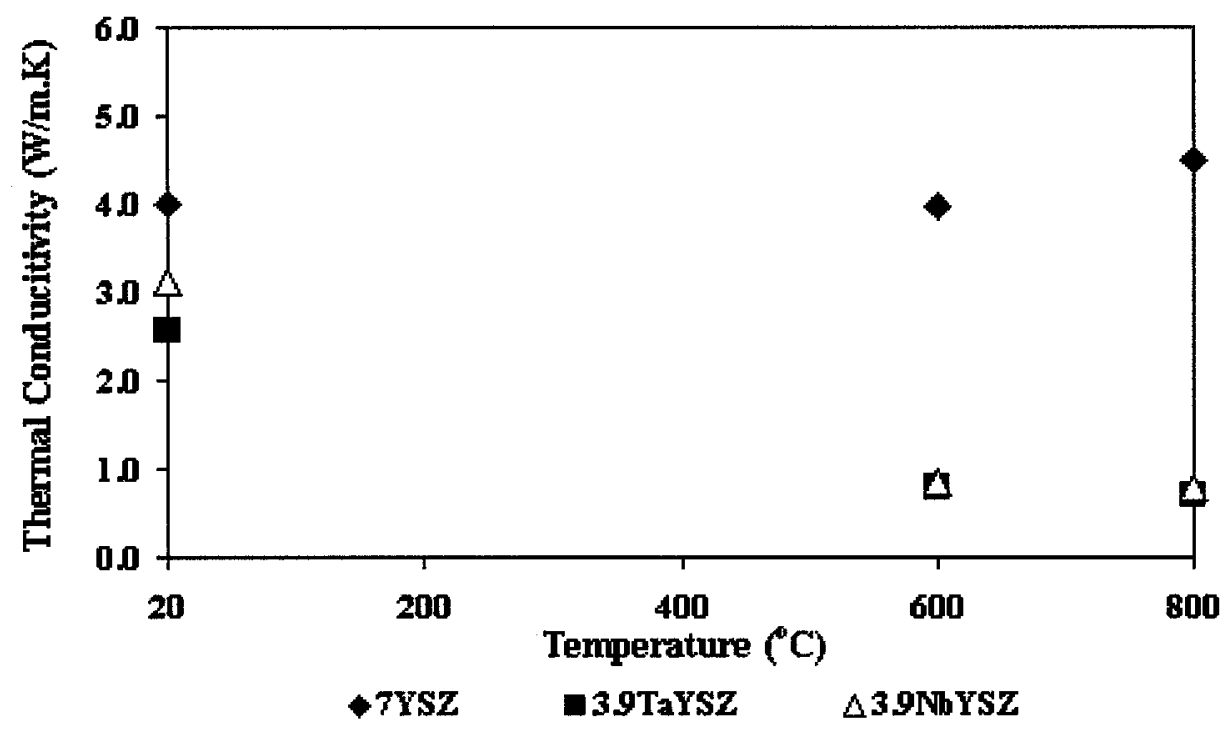

Figure 84 Variations of thermal conductivity as a function of temperatures for 7YSZ and 3.9MYSZ $(\mathrm{M}=\mathrm{Ta}$ and $\mathrm{Nb})$ samples.

From these figures it can be seen that at room temperature, all the doped materials have lower thermal conductivities than 7YSZ. Among the 5MYSZ $(\mathrm{M}=\mathrm{Cr}, \mathrm{Sc}, \mathrm{Yb}$ and $\mathrm{Ce})$ samples, the thermal conductivity of 5YbYSZ is the lowest and that of 5CeYSZ is the highest. This can be easily understood. Based on thermal conductivity theory, the addition of $\mathrm{M}^{3+}$ or $\mathrm{Ce}^{4+}$ to 7YSZ creates either more oxygen vacancies or more substitutional atom defects, which therefore increases phonon scattering centers. Among these values, 5CeYSZ has a relatively higher thermal conductivity than that of the 5MYSZ $(\mathrm{M}=\mathrm{Cr}, \mathrm{Sc}$, and $\mathrm{Yb})$ samples although the mole percentage of $\mathrm{Ce}^{4+}$ ions is the same as $\mathrm{M}^{3+}$ ions. Since the addition of $\mathrm{Ce}^{4+}$ to $7 \mathrm{YSZ}$ does not create additional oxygen vacancies (some oxygen vacancies are believed to have formed due to the formation of a small amount of $\mathrm{Ce}^{3+}$ during the sintering process), it is therefore thought that the phonon scattering by oxygen vacancies is more effective than that due to the substitutional $\mathrm{Ce}^{4+}$ 
ions. Likewise, the doped $\mathrm{Ta}^{5+}-$ or $\mathrm{Nb}^{5+}-7 \mathrm{YSZ}$ samples were also observed to have lower thermal conductivities at both room temperature and elevated temperatures than 7YSZ. Especially at high temperatures, thermal conductivities of these two materials dropped rapidly. For these two materials, the oxygen vacancies created by $\mathrm{Y}^{3+}$ could be annihilated by $\mathrm{Ta}^{5+}$ or $\mathrm{Nb}^{5+}$ because of the compensation for electrical charge. The reductions in thermal conductivity may be caused by the phonon scattering centers formed by the defect clusters due to the differences in ionic radius or mass between the pentavalent dopant cations $\mathrm{Ta}^{5+}$ and $\mathrm{Nb}^{5+}$ and the host cation $\mathrm{Zr}^{4+}$. Besides, the thermal conductivity of $\mathrm{Ta}^{5+}$ co-doped 7YSZ is slightly lower than that of $\mathrm{Nb}^{5+}$ co-doped 7YSZ, which may be caused by the mass differences since the atomic mass of Ta is twice of that of $\mathrm{Nb}$. In addition, according to the phase transformation phenomenon, after the samples were cooled down to room temperature from testing, both $3.9 \mathrm{TaYSZ}$ and $3.9 \mathrm{NbYSZ}$ discs were found to have crumbled to powdery states. Thus these two samples became loose structures with more porosity at high temperatures such that they resulted in higher thermal insulation.

At high temperatures, the thermal conductivities for 7YSZ, 5CrYSZ, 5ScYSZ and $5 \mathrm{CeYSZ}$ were found to be higher than at room temperature. These high values for these samples resulted from their high specific heats at high temperatures; and the high specific heat may arise from the loss of oxygen or phase transition. Another explanation is that at high temperatures, the atomic defect scatterings were dominant, thus the thermal conductivities were not sensitive to the temperature.

In summary, compare the thermal conductivity results for all the doped 7YSZ materials, 
the $\mathrm{Yb}_{2} \mathrm{O}_{3}$ co-doped $7 \mathrm{YSZ}$ was the most effective dopant in reducing the thermal conductivity due to the added oxygen vacancies and its larger ionic radius and atomic weight. On the other hand, the $\mathrm{CeO}_{2}$ co-doped $7 \mathrm{YSZ}$ shows the least effec on the reduction in thermal condcutivity. It is suggested that larger and heavier doping ions with lower valence are to be selected in order to further reduce the thermal conductivity of 7YSZ. 


\section{Modeling of Thermal Conductivity}

It has been well known that the addition of metal oxide to zirconia creates point defects, such as oxygen vacancies, substitution of host atoms, etc. These point defects increase phonon scattering centres and thus result in an increase in thermal resistance, as seen in the previous literature review. According to thermal conductivity theory, the scattering effect from each point defect is associated with the type of point defect (oxygen vacancy or substitution of the host atom) and the characteristics of the dopant cations including mass, ionic radius, valence, etc. Various models to predict how the thermal resistance resulting from the dopants in the zirconia-based ceramics quantitatively change with the dopants parameters have been published $[30,74,75,128,129,130]$. However, these models were based on the assumption that each point defect such as an oxygen vacancy or substitutional atom was isolated, and the phonon scattering from one point defect was not related with another. In fact, for $\mathrm{ZrO}_{2}$ doped with $\mathrm{MO}$ or $\mathrm{M}_{2} \mathrm{O}_{3}$, oxygen vacancies created by the metal cations will always be closely coupled to the dopant cations. This has been confirmed by M. O. Zacate et al. [131]. These workers studied the formation of defect clusters in $\mathrm{M}_{2} \mathrm{O}_{3}$ - doped cubic $\mathrm{ZrO}_{2}$ and found that, when the $\mathrm{M}_{2} \mathrm{O}_{3}$ oxides were added to $\mathrm{ZrO}_{2}$, the oxygen vacancies always occupied the sites that were first neighbours with respect to small dopants, or second neighbours with respect to large dopants. This dopant cation and the resulting oxygen vacancy in the neighbour formed a local defect cluster, which was of the order of the lattice parameter of $\mathrm{ZrO}_{2}$ and resulted in a local distortion of the lattice. For example, in $\mathrm{Y}_{2} \mathrm{O}_{3}$ doped $\mathrm{ZrO}_{2}$ material, the most energetically stable configurations for the defects of oxygen vacancies and dopant cations 
were $\left(M_{Z r}^{\prime}: V_{o}^{* \bullet}\right)^{\bullet}$ clusters or $\left(2 M_{Z r}^{\prime}: V_{o}^{* \bullet}\right)^{\times}$clusters instead of atomic defects $M_{Z r}^{\prime}$ or $V_{o}^{* *}$. These defect clusters had been confirmed using high-resolution transmission electron microscopy (TEM) by D. Zhu et al. [87] in the study of the characterizations of $\mathrm{Nd}, \mathrm{Gd}$, and $\mathrm{Yb}$ rare-earth doped zirconia-yttria thermal barrier coatings. Apparently, the phonon scattering effect caused by defect clusters is different from that caused by the single atomic defect. Therefore, to accurately predict how thermal conductivity varies with the dopant parameters, the defect cluster should be considered as one combined scattering source. In the present study, a semi-empirical model of thermal conductivity varying with the dopants for $\mathrm{M}_{2} \mathrm{O}_{3}$ or $\mathrm{M}_{2} \mathrm{O}_{5}$ doped $7 \mathrm{YSZ}$ materials is proposed, based on existing thermal conductivity theory and the assumption that the defect clusters may be considered as phonon scatterings sources. Some published experimental data have been adopted to evaluate the parameters in the model. Finally, the results calculated from the doped materials: $3.9 \mathrm{TaYSZ}, 3.9 \mathrm{NbYSZ}, 5 \mathrm{ScYSZ}, 5 \mathrm{YbYSZ}$ and $7 \mathrm{YSZ}$ samples using this model are compared with the experimental results shown in Chapter 5 to evaluate the validality of the model.

\subsection{Thermal Resistance of Zirconia Based Materials}

The evaluation of thermal conductivity of zirconia based materials is based on the Klemens-Callaway theory described in 2.6.1, and (Eq. 37) and (Eq. 38) are used. Most thermal conductivity models published were established on the assumption that the material was a single-phase crystal with homogeneously distributed point defects $[127$, $155,128,129]$. However, the actual crystal structures may include more than one phase 
with each phase having different fractions of defects; and the measured thermal conductivities at room temperature are the combination of the thermal conductivities of these multiple phases. Besides, the intrinsic thermal conductivity is also dependent on the crystal structures, since the phonon dispersion curve in reciprocal space is determined by the crystal structure. For zirconia-based ceramics, the room temperature structure may consist of monoclinic, tetragonal or cubic phases, depending on the dopant content and heat treatment conditions. The thermal resistance for zirconia based materials was therefore modified as:

$W=\sum_{i} C_{i} W_{i}$

where $i=m, t$, or $c$. And $C_{m}, C_{t}$, and $C_{c}$ denote the contents of monoclinic, tetragonal and cubic phases, respectively; and $W_{m}, W_{t}$, and $W_{c}$ denote the thermal resistance of these phases, respectively. The $W_{i}$ can be obtained from the following relationship:

$W_{i}=W_{P i}+\frac{\Omega_{0} \theta}{6 \hbar v_{G}^{2}} \Gamma_{i}=W_{P i}+B \cdot \Gamma_{i}$

(Eq. 80)

where $W_{P m}, W_{P t}$, and $W_{P c}$ are the intrinsic thermal resistance of monoclinic, tetragonal and cubic phases, respectively. For doped $\mathrm{ZrO}_{2}$ based materials, the transformation from cubic to tetragonal phase did not result in a pronounced volume change, thus, the intrinsic thermal resistances for these two phases could be assumed to be the same, denoted as $W_{P_{c}}$, for the purpose of simplification. $B$ is expressed as: 


$$
B=\frac{\Omega_{0} \theta}{6 \hbar v_{G}^{2}}
$$

(Eq. 81)

where $\Omega_{0}$ is the volume per atom of the crystal. Considering that there are three atoms in one $\mathrm{ZrO}_{2}$ molecule, the average atomic volume $\Omega_{0}$ is given from:

$$
\Omega_{0}=\frac{M}{3 \times 6.023 \times 10^{-23} \rho}
$$

The group velocity of the phonons $v_{G}$ may vary in doped materials and it will be calculated using the relationship derived by D.Clarke [132]:

$$
v_{G}=0.87 \sqrt{\frac{E}{\rho}}
$$

where $E$ is the elastic modulus and $\rho$ is the density, measured in the present experiment for zirconia based material.

The Debye temperature $\theta$ is evaluated using its definition [68]:

$$
\theta=\frac{\hbar v_{G}}{k_{B}}\left(6 \pi^{2} \frac{N_{A}}{V}\right)^{\frac{1}{3}}=\frac{\hbar v_{G}}{k_{B}}\left(6 \pi^{2} \frac{1}{a_{0}^{3} / 4}\right)^{\frac{1}{3}}=6.187 \frac{\hbar}{k_{B}} \frac{v_{G}}{a_{0}}
$$

In the analysis of thermal conductivity data, to determine the scattering factor $\Gamma$, it is necessary first to estimate all the scattering centers. In the present study, the following doped zirconia systems are studied. 
1. In the $\mathrm{M}_{2} \mathrm{O}_{3}$ doped $\mathrm{ZrO}_{2}$ binary system, the point defects consisted of the substituted dopant cations $\mathrm{M}^{3+}$ and the oxygen vacancies created by those ions and the scattering center is assumed to be a defect cluster of $\left(2 M_{Z r}^{\prime}: V_{o}^{* \bullet}\right)^{\times}$. The defect chemistry is described using the Kroger-Vink notation [133]:

$M_{2} O_{3} \rightarrow 2 M_{Z r}^{\prime}+V_{o}^{\bullet \bullet}+2 O_{o}^{x}$

2. In the $\mathrm{M}_{2} \mathrm{O}_{3}$ and $\mathrm{Y}_{2} \mathrm{O}_{3}$ co-doped $\mathrm{ZrO}_{2}$ ternary system, the point defects are composed of both $\mathrm{M}^{3+}$ and $\mathrm{Y}^{3+}$ dopant cations as well as oxygen vacancies created by those cations, which resulted in the scattering centers formed by the configurations of the defect clusters $\left(M_{Z r}^{\prime}: Y_{z r}^{\prime}: V_{O}^{\bullet \bullet}\right)^{\times},\left(2 M_{Z r}^{\prime}: V_{O}^{*}\right)^{\times}$or $\left(2 Y_{z r}^{\prime}: V_{O}^{* \bullet}\right)^{\times}$. The Kroger-Vink notation is expressed as $\frac{1}{2} M_{2} O_{3}+\frac{1}{2} Y_{2} O_{3} \rightarrow M_{Z r}^{\prime}+Y_{Z r}^{\prime}+V_{o}^{* *}+2 O_{o}^{x} \quad(\mathrm{M}=\mathrm{Cr}, \mathrm{Sc}$ and $\mathrm{Yb})$

3. In the $\mathrm{M}_{2} \mathrm{O}_{5}$ and $\mathrm{Y}_{2} \mathrm{O}_{3}$ co-doped $\mathrm{ZrO}_{2}$ ternary system, the oxygen vacancies created by $\mathrm{Y}^{3+}$ are annihilated by the addition of $\mathrm{M}^{5+}$ cations, as suggested by published literature [86] and supported by the XRD results of the present work. If the dopant fractions of both $\mathrm{M}^{5+}$ and $\mathrm{Y}^{3+}$ are the same, there is no oxygen vacancy present. The scattering source is therefore assumed to be a defect cluster of $\left(M_{Z r}^{*}: Y_{Z r}^{\prime}\right)$. The Kroger-Vink notation for this situation is given:

$$
\frac{1}{2} M_{2} O_{5}+\frac{1}{2} Y_{2} O_{3} \rightarrow M_{Z r}^{\cdot}+Y_{Z r}^{\prime}+4 O_{o}^{x} \quad(\mathrm{M}=\mathrm{Ta} \text { and } \mathrm{Nb})
$$

If the fraction of $\mathrm{Y}^{3+}$ cations is more than that of $\mathrm{M}^{5+}$ cations, the two kind of clusters of $\left(M_{Z r}^{\bullet}: Y_{Z r}^{\prime}\right)$ and $\left(2 Y_{Z r}^{\prime}: V_{O}^{\ddot{O}}\right)^{\times}$will be the scattering centers. 
From the discussion above, the phonon scattering factor for the $\mathrm{M}_{2} \mathrm{O}_{3}$ or $\mathrm{M}_{2} \mathrm{O}_{5}$ doped $\mathrm{ZrO}_{2}$ is assumed to be determined by defect clusters, which result from the differences in local masses and local volumes between the cluster and the perfect crystal. The new definition for the phonon scattering factor $\Gamma$ is described as below:

$$
\Gamma_{i}=\sum_{j} x_{j}\left[\varepsilon_{j 1} \cdot\left(\frac{\Delta M_{j}}{M}\right)^{2}+\varepsilon_{j 2} \cdot\left(\frac{\Delta \delta_{j}}{\delta}\right)^{2}\right]
$$

where $x_{j}$ is the fractional concentration of the $j^{\text {th }}$ type of defect cluster; $\Delta M_{j}$ is the difference in average atomic mass between the $j^{\text {th }}$ type of defect cluster and the perfect crystal; $\Delta \delta_{j}$ is the difference in average atomic radius between the $j^{\text {th }}$ type of defect cluster and the perfect crystal; $M$ and $\delta$ are the average atomic mass and radius of the virtual crystal, respectively; and $\varepsilon_{j 1}$ and $\varepsilon_{j 2}$ are the adjustable parameter for the average atomic mass and radius differences between the defect cluster and the host, respectively.

To determine the adjustable parameters $\varepsilon_{j 1}$ and $\varepsilon_{j 2}$, the experimental results of Y-PSZ, Dy-PSZ, and Yb-PSZ published in Ref. [30] and the experimental results of N4 and T9 in Ref. [86] were used. In these doped materials, the defect clusters could be classified into two types: $\left(2 M_{Z r}^{\prime}: V_{o}^{* *}\right)^{\times}$, where $\mathrm{M}=\mathrm{Y}$, or $\mathrm{Dy}$, or $\mathrm{Yb}$; and $\left(M_{Z r}^{\cdot}: Y_{Z r}^{\prime}\right)^{\times}$, where $\mathrm{M}=\mathrm{Ta}$ or $\mathrm{Nb}$. Thus, a total of four adjustable parameters need to be determined.

In the Y-PSZ, Dy-PSZ and Yb-FSZ samples [30], only $t$ ' phase were observed at room temperature. The defect cluster for Y-PSZ is $\left(2 Y_{Z r}^{+}: V_{o}^{*}\right)^{\times}$, for Dy-PSZ is $\left(2 D y_{Z r}^{\prime}: V_{o}^{\bullet}\right)^{\times}$, and for $\mathrm{Yb}-\mathrm{FSZ}$ is $\left(2 \mathrm{Yb} b_{\mathrm{Zr}}^{\prime}: V_{O}^{\bullet \bullet}\right)^{\times}$. They are the same type of clusters and can be used to 
determine the adjustable parameters $\varepsilon_{11}$ and $\varepsilon_{12}$. The (Eq. 80) for these doped materials is rewritten as:

$W_{q}=W_{P c}+B_{Z r o 2} \Gamma_{q}$

where $q$ represents Y-PSZ, Dy-PSZ, or Yb-FSZ, and:

$$
\Gamma_{q}=x_{q}\left[\varepsilon_{11}\left(\frac{\Delta M_{1 q}}{M}\right)^{2}+\varepsilon_{12}\left(\frac{\Delta \delta_{1 q}}{\delta}\right)^{2}\right]
$$

In the N4 and T9 samples [86], both tetragonal and cubic phases existed at room temperature. Within the tetragonal phase, the oxygen vacancies were annihilated due to the presence of the same mole percentages of $\mathrm{Nb}_{2} \mathrm{O}_{5}$ (or $\mathrm{Ta}_{2} \mathrm{O}_{5}$ ) and $\mathrm{Y}_{2} \mathrm{O}_{3}$. Thus the defect cluster type within the $t$-phase was $\left(M_{Z r}^{\cdot}: Y_{Z r}^{\prime}\right)^{\times}$, and the crystal structures in the $t$ phase for $\mathrm{N} 4$ and $\mathrm{T} 9$ are therefore assumed to be the same as that of pure $\mathrm{ZrO}_{2}$ in the monoclinic phase; and their intrinsic thermal resistance is that of $\mathrm{m}-\mathrm{ZrO}_{2}$ and to be $W_{P m}$. The defect cluster of $\left(M_{Z r}^{*}: Y_{Z r}^{\prime}\right)^{\times}$is used to determine the corresponding adjustable parameters $\varepsilon_{21}$ and $\varepsilon_{22}$. Within the cubic phase, only $\mathrm{Y}_{2} \mathrm{O}_{3}$ was observed and the defect cluster was therefore only the type of $\left(2 Y_{Z r}^{\prime}: V_{o}^{\bullet \bullet}\right)^{x}$. The thermal resistances for these two samples can be expressed as:

$$
W_{q}=C_{t} \cdot\left(W_{P m}+B_{Z r O 2} \Gamma_{t q}\right)+C_{c} \cdot\left(W_{P t / c}+B_{Z r O 2} \Gamma_{c q}\right)
$$

where $q$ represented $\mathrm{N} 4$ and $\mathrm{T} 9$, respectively; and: 


$$
\begin{aligned}
& \Gamma_{t q}=x_{t q}\left[\varepsilon_{21}\left(\frac{\Delta M_{2 q}}{M}\right)^{2}+\varepsilon_{22}\left(\frac{\Delta \delta_{2 q}}{\delta}\right)^{2}\right] \\
& \Gamma_{c q}=x_{c q}\left[\varepsilon_{11}\left(\frac{\Delta M_{1 q}}{M}\right)^{2}+\varepsilon_{12}\left(\frac{\Delta \delta_{1 q}}{\delta}\right)^{2}\right]
\end{aligned}
$$

To solve equations (Eq. 86) to (Eq. 89) for each sample, the value of $B_{\mathrm{ZrO}_{2}}$ for $\mathrm{ZrO}_{2}$ was calculated using (Eq. 83) $\sim\left(\right.$ Eq. 82) and which gave $B_{Z r O 2}=1.058$. In addition, the thermal conductivity of pure $\mathrm{ZrO}_{2}$ measured at the room temperature by $\mathrm{R}$. Mevrel, et al [129] was adopted as $W_{P m}$ for the calculation, and the value is shown in Figure 85 .

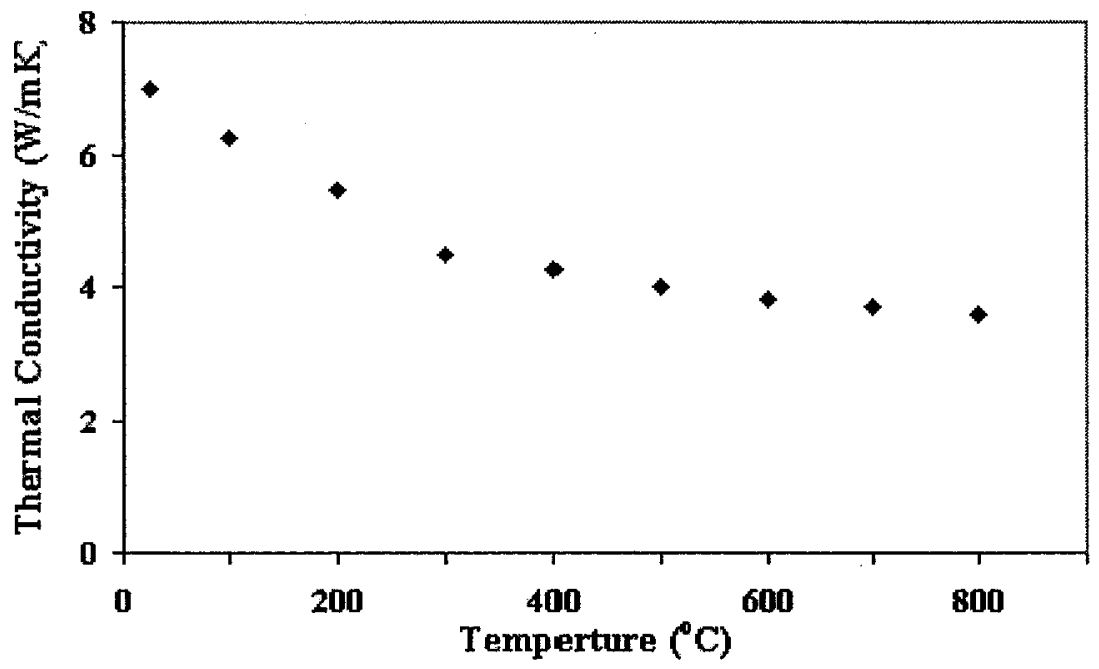

Figure 85 Thermal conductivities of pure $m-\mathrm{ZrO}_{2}[129]$ as a function of temperature.

At room temperature, the intrinsic thermal conductivity $W_{P c}$ for the cubic or tetragonal $\mathrm{ZrO}_{2}$ can not be measured since the pure $\mathrm{ZrO}_{2}$ exists only at the monoclinic phase. Thus, except for the values of the adjustable parameters $\varepsilon_{11}, \varepsilon_{12}, \varepsilon_{21}$, and $\varepsilon_{22}$ shown in (Eq. 87) and (Eq. 89), another parameter $W_{P c}$ also needs to be determined. The differences in 197 
masses $\Delta M_{j}$ and differences in ionic radii $\Delta \delta_{j}$ between the defect clusters and the host cations were calculated and these data are given in Table 20 . In addition, the values of the contents of each type of defect cluster within each phase present, the phase contents as well as the measured thermal conductivities are also listed in Table 20. Substituting the data shown in Table 20 into (Eq. 86) to (Eq. 89) and solving these equations, the values of $\varepsilon_{11}, \varepsilon_{12}, \varepsilon_{21}$ and $\varepsilon_{22}$ as well as $W_{P c}$ were obtained: $\varepsilon_{11}=5.00 ; \varepsilon_{12}=246.82$; $\varepsilon_{21}=-2.42 ; \varepsilon_{22}=695.18 ;$ and $W_{P c}=0.3414$.

Table 20 The data for determining parameters $\varepsilon_{11}, \varepsilon_{12}, \varepsilon_{21}, \varepsilon_{22}$ and $W_{P c}$.

\begin{tabular}{|c|c|c|c|c|c|c|}
\hline Sample & $\begin{array}{c}\text { Thermal } \\
\text { conductivity } \\
(\mathrm{W} / \mathrm{mK})\end{array}$ & Cluster type & $\Delta M_{j}$ & $\Delta \delta_{j}$ & $\begin{array}{l}\text { Cluster content } \\
\text { (mol\%) }\end{array}$ & $\begin{array}{l}\text { Phase content } \\
\text { (mol\%) }\end{array}$ \\
\hline Y-PSZ [30] & 2.6 & $\mathrm{t}^{\prime}-\left(2 Y_{z r}^{\prime}: V_{o}^{* \bullet}\right)^{\mathrm{x}}$ & -3.42 & 0.0050 & 4.49 & 100 \\
\hline Dy-PSZ [30] & 2.2 & $\mathbf{t}^{\prime}-\left(2 D y_{z r}^{\prime}: V_{o}^{* *}\right)^{x}$ & 21.11 & 0.0062 & 4.00 & 100 \\
\hline Yb-FSZ [30] & 1.7 & $\mathbf{t}^{\prime}-\left(2 Y b_{Z r}^{\prime}: V_{o}^{\bullet \bullet}\right)^{\times}$ & 24.63 & 0.0020 & 11.98 & 100 \\
\hline \multirow{3}{*}{ N4 [86] } & \multirow{3}{*}{2.1} & $\mathrm{t}-\left(N b_{z r}^{*}: Y_{z r}^{\prime}\right)^{\times}$ & -0.08 & -0.0050 & 13.8 & \multirow{2}{*}{58.90} \\
\hline & & $\mathrm{t}-\left(2 Y_{z r}^{\prime}: V_{o}^{\bullet \bullet}\right)^{\times}$ & -3.42 & 0.0050 & 0.55 & \\
\hline & & $\mathrm{c}-\left(2 Y_{Z r}^{\prime}: V_{o}^{* \bullet}\right)^{\times}$ & -3.42 & 0.0050 & 10.4 & 40.10 \\
\hline \multirow{3}{*}{ T9 [86] } & \multirow{3}{*}{2.05} & $\mathrm{t}-\left(T a_{z r}^{*}: Y_{z r}^{*}\right)^{x}$ & 14.58 & -0.0055 & 12.92 & \multirow{2}{*}{61.84} \\
\hline & & $\mathrm{t}-\left(2 Y_{Z r}^{\prime}: V_{o}^{* *}\right)^{x}$ & -3.42 & 0.0050 & 0.94 & \\
\hline & & $\mathrm{c}-\left(2 Y_{Z r}^{\prime}: V_{o}^{* \ddot{\prime}}\right)^{\times}$ & -3.42 & 0.0050 & 10.20 & 38.16 \\
\hline
\end{tabular}

Substituting the obtained parameters into(Eq. 79), (Eq. 80) and (Eq. 85), the model of thermal resistance was derived as:

$W=C_{m} \cdot\left(0.143+1.058 \cdot \Gamma_{m}\right)+C_{t} \cdot\left(0.3414+1.058 \cdot \Gamma_{t}\right)+C_{c} \cdot\left(0.3414+1.058 \cdot \Gamma_{c}\right)$

(Eq. 90) 
The scattering factors within each phase could then be rewritten as:

$$
\Gamma=\sum_{j} x_{1 j}\left[5.00 \cdot\left(\frac{\Delta M_{1 j}}{M}\right)^{2}+246.82 \cdot\left(\frac{\Delta \delta_{1 j}}{\delta}\right)^{2}\right]+\sum_{j} x_{2 j}\left[-2.42 \cdot\left(\frac{\Delta M_{1 j}}{M}\right)^{2}+695.18 \cdot\left(\frac{\Delta \delta_{1 j}}{\delta}\right)^{2}\right]
$$

\subsection{Calculation of Thermal Conductivities of the Doped Zirconia Based Materials Using the Thermal Resistance Model}

To validate the thermal resistance model, the thermal resistances of $7 \mathrm{YSZ}, 5 \mathrm{CrYSZ}$, 5ScYSZ, 5YbYSZ, 3.9TaYSZ, and 3.9NbYSZ were calculated. The data required for calculation for each doped material are listed in Table 21. The calculated thermal resistances and the corresponding thermal conductivities are listed in Table 22. For comparison, the measured thermal conductivities and the relative differences between the calculated and measured results are also listed in Table 22 and illustrated in Figure 86.

The thermal resistance (and therefore thermal conductivity) of the sample 5CeYSZ was not calculated using the proposed model, since in the $5 \mathrm{CeYSZ}$ system, the defects consisted of both defect clusters $\left(2 Y_{Z r}^{\prime}: V_{O}^{\bullet \bullet}\right)^{\times}$and substituted $\mathrm{Ce}^{4+}$ defects. The cross section of phonon scattering from the substituted $\mathrm{Ce}^{4+}$ defect is different from the defect clusters discussed above, thus another two adjustable parameters $\varepsilon_{31}$ and $\varepsilon_{32}$ for the

substituted $\mathrm{Ce}^{4+}$ defect are required. However, the experimental data for determining these parameters are not available in the current study. Thus, the model proposed cannot be applied to 5CeYSZ. 
Table 21 Data for calculation of thermal resistance using the established model

\begin{tabular}{|c|c|c|c|c|c|}
\hline Sample & Cluster type & $\Delta M_{j}$ & $\Delta \delta_{j}$ & $\begin{array}{c}\text { Cluster content } \\
(\mathrm{mol} \%)\end{array}$ & $\begin{array}{c}\text { Phase content } \\
\qquad(\mathrm{mol} \%)\end{array}$ \\
\hline \multirow{2}{*}{ 7YSZ } & $\mathrm{m}-\left(2 Y_{z r}^{\prime}: V_{o}^{*}\right)^{x}$ & -3.42 & 0.0050 & 1.52 & 46.00 \\
\hline & $\mathrm{c}-\left(2 Y_{Z r}^{\prime}: V_{o}^{\ddot{*}}\right)^{\mathrm{x}}$ & -3.42 & 0.0050 & 5.99 & 54.00 \\
\hline \multirow{2}{*}{ 3.9TaYSZ } & $\mathrm{m}-\left(T a_{Z r}^{\cdot}: Y_{Z r}^{\prime}\right)^{x}$ & 14.58 & -0.0055 & 7.58 & 100 \\
\hline & $\mathrm{m}-\left(2 Y_{z r}^{\prime}: V_{o}^{*-*}\right)^{x}$ & -3.42 & 0.0050 & 0.0059 & 100 \\
\hline \multirow{2}{*}{ 3.9NbYSZ } & $\mathrm{m}-\left(N b_{Z r}^{\bullet}: Y_{Z r}^{\prime}\right)^{\times}$ & -0.08 & 0.0050 & 7.58 & 96.52 \\
\hline & 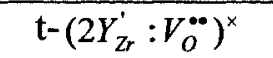 & -3.42 & 0.0050 & 0.0059 & 3.48 \\
\hline \multirow{3}{*}{ 5CrYSZ } & $\mathrm{m}-\left(2 Y_{\mathrm{Zr}}^{\prime}: V_{o}^{* \bullet}\right)^{\mathrm{x}}$ & -3.42 & 0.0050 & 1.52 & 51.52 \\
\hline & $\mathrm{c}-\left(C r_{z r}^{\prime}: Y_{z r}^{\prime}: V_{o}^{*}\right)^{x}$ & -9.57 & -0.0080 & 1.25 & \multirow{2}{*}{48.48} \\
\hline & $\mathrm{c}-\left(2 Y_{Z r}^{\prime}: V_{o}^{\prime *}\right)^{x}$ & -3.42 & 0.0050 & 5.64 & \\
\hline \multirow{4}{*}{$5 \mathrm{ScYSZ}$} & $\mathrm{m}-\left(S c_{z r}^{\prime}: Y_{z r}^{\prime}: V_{o}^{* *}\right)^{\times}$ & -10.74 & -0.0035 & 1.20 & \multirow{2}{*}{5.70} \\
\hline & $\mathrm{m}-\left(2 Y_{Z r}^{\prime}: V_{o}^{\ddot{*}}\right)^{\times}$ & -3.42 & 0.0050 & 0.33 & \\
\hline & $\mathrm{c}-\left(S c_{z r}^{\prime}: Y_{z r}^{\prime}: V_{o}^{* *}\right)^{x}$ & -10.74 & -0.0035 & 5.25 & \multirow{2}{*}{94.30} \\
\hline & $\mathrm{c}-\left(2 Y_{Z r}^{\prime}: V_{o}^{\prime *}\right)^{x}$ & -3.42 & 0.0050 & 1.41 & \\
\hline \multirow{2}{*}{ 5YbYSZ } & c- $\left(Y b_{z r}^{\prime}: Y_{Z r}^{\prime}: V_{o}^{* *}\right)^{x}$ & 10.61 & 0.0035 & 5.01 & \multirow{2}{*}{100} \\
\hline & $\mathrm{c}-\left(2 Y_{Z r}^{\prime}: V_{o}^{* \bullet}\right)^{x}$ & -3.42 & 0.0050 & 1.34 & \\
\hline
\end{tabular}

The comparison listed in Table 22 and shown in Figure 86 indicates that the differences between the calculated and measured thermal conductivities were within $10 \%$ except $7 \mathrm{YSZ}$ and $5 \mathrm{CrYSZ}$. The larger scatter for $5 \mathrm{CrYSZ}$ may be caused by the inaccurate $\mathrm{Cr}^{3+}$ content measurement. For the 5CrYSZ, 5ScYSZ, 5YbYSZ and 3.9TaYSZ, the calculated thermal conductivities were a little higher than the measured values. These differences might arise from the error in porosity measurement. The measured samples might have 
higher porosity, which resulted in the lower thermal conductivities. However, the calculated thermal conductivities could still provide a trend for the metal oxide doped zirconia based materials, and therefore provide a guideline for the selection of thermal barrier coating materials.

Table 22 Thermal conductivities of the calculated and measured results

\begin{tabular}{|l|l|l|l|l|}
\hline Sample ID & $\begin{array}{c}\text { Calculated } \mathrm{W}_{\text {cal. }} \\
(\mathrm{mK} / \mathrm{W})\end{array}$ & $\begin{array}{c}\text { Calculated } \lambda_{\text {cal. }} \\
(\mathrm{W} / \mathrm{mK})\end{array}$ & $\begin{array}{c}\text { Measured } \lambda_{\text {mea. }} \\
(\mathrm{W} / \mathrm{mK})\end{array}$ & $\left(\lambda_{\text {mea. }}-\lambda_{\text {cal }}\right) / \lambda_{\text {mea. }}$ \\
\hline 7YSZ & 0.29 & 3.42 & 3.99 & 0.14 \\
\hline $3.9 \mathrm{TaYSZ}$ & 0.35 & 2.80 & 2.57 & -0.09 \\
\hline $3.9 \mathrm{NbYSZ}$ & 0.34 & 2.94 & 3.13 & 0.06 \\
\hline $5 \mathrm{CrYSZ}$ & 0.29 & 3.46 & 2.70 & -0.28 \\
\hline 5 ScYSZ & 0.38 & 2.60 & 2.35 & -0.11 \\
\hline 5 YbYSZ & 0.39 & 2.53 & 2.30 & -0.10 \\
\hline
\end{tabular}

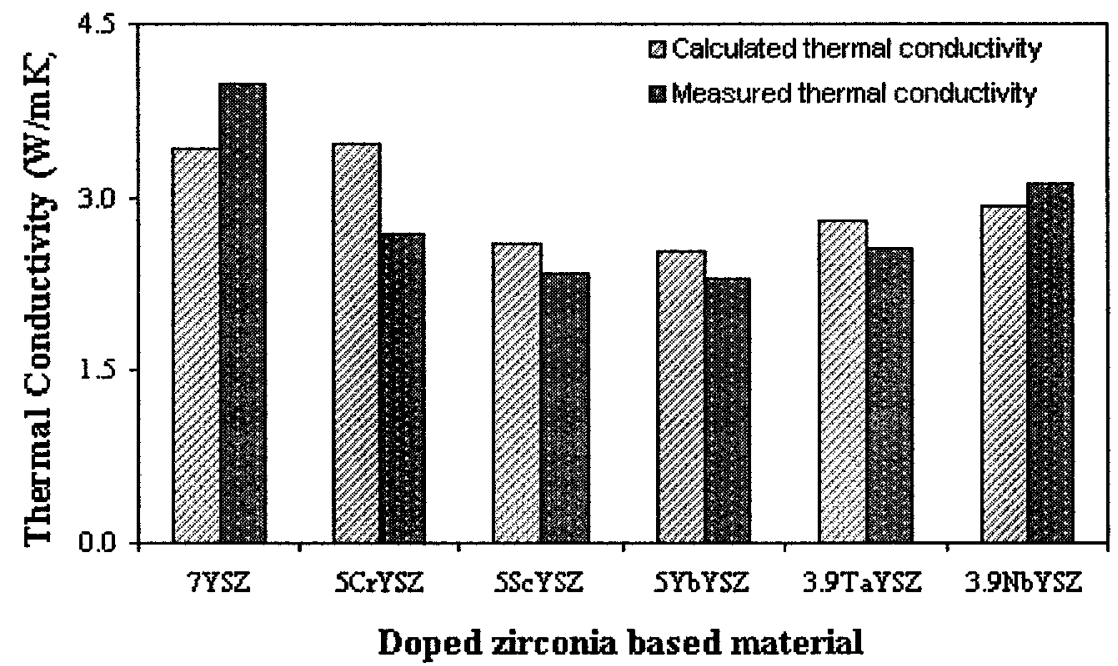

Figure 86 Comparison of thermal conductivities between the calculated results and the measured results. 


\section{Multiple Layered Thermal Barrier Coatings Design}

As summarized in the literature review, the heat transfer in ceramic materials is achieved by two mechanisms: thermal conduction and thermal radiation. In the previous chapter, it has been shown that the thermal conduction in ceramic materials can be effectively reduced by employing doping methods. In this chapter, the thermal radiation issue will be tackled using the design of a multiple layered coating system. To achieve a significant reduction in temperature increase at the substrate caused by radiation, the concept of using multiple layered coatings containing several stacks to effectively reflect radiation within a wavelength range of $0.3 \sim 5 \mu \mathrm{m}[78]$ is proposed and will be described in the following sections.

\subsection{Design of Multiple Layered Stacks with High Reflectance}

The proposed structure consists of several sets of highly reflective multiple layered stacks, with each stack being specifically designed to reflect a targeted range of wavelength. A broadband reflection for the required wavelength range can be obtained using a sufficient number of stacks. To achieve high reflectance for each wavelength range, each stack must have multiple layers of ceramic materials with alternating high and low refractive indices, and the optical thickness of each layer must be equal to a quarter wavelength in order to satisfy the condition of multiple-beam interference [134]. Since the radiation with shorter wavelengths will be scattered much more strongly [135], the stack reflecting the shortest wavelength range should be placed on top of the multiple layer stacks, and the stack reflecting the longest wavelength range placed below the other 
multiple layers. Figure 87 shows schematically how the radiation is reflected or transmitted by these multiple layers.

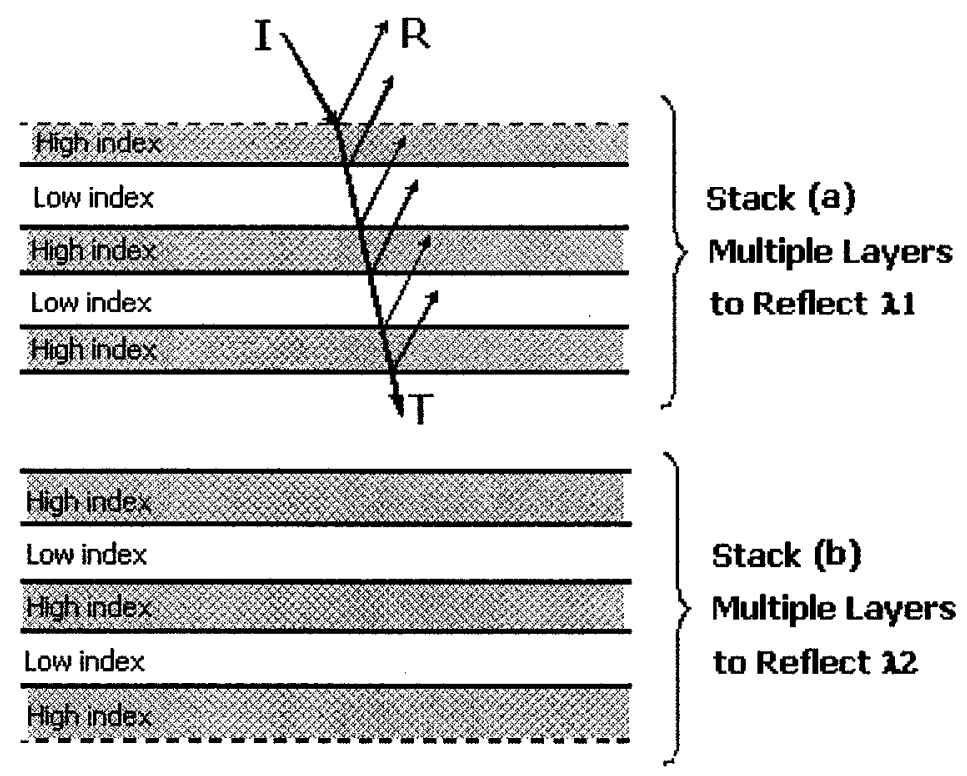

Figure 87 Schematic of multiple layered coating structure containing two stacks $(T=$ transmitted, $\mathrm{R}=$ reflected and $\mathrm{I}=$ incoming radiations)

\subsubsection{Fundamental Concept of Multiple Beam Interference}

The multiple layered stacks can be designed by calculating the physical thickness of each layer in one stack using [134]:

$d_{H}(\lambda)=\lambda /\left(4 n_{H}\right)$

$d_{L}(\lambda)=\lambda /\left(4 n_{L}\right)$

where $d_{H}$ and $d_{L}$ are thicknesses for the alternating layers within the stack, and $n_{H}$ and $n_{L}$ are the refractive indices of the alternating layers. $H$ denotes the layer with high 
reflective index and $L$ denotes the layer with low reflective index; and $\lambda$ is the radiation wavelength.

Assuming that the scattering of the radiation in the high reflectance stacks is negligible; the reflectance $\rho_{\lambda}$ and transmittance $\tau_{\lambda}$ of the multiple layered coatings, for one wavelength range, is then given by:

$\rho_{\lambda}=\left(\frac{\eta_{0} B-C}{\eta_{0} B+C}\right)\left(\frac{\eta_{0} B-C}{\eta_{0} B+C}\right)^{*}$

$$
\tau_{\lambda}=\frac{4 \eta_{0} \operatorname{Re}\left(\eta_{m}\right)}{\left(\eta_{0} B+C\right)\left(\eta_{0} B+C\right)^{*}}
$$

where the term $\left(\frac{\eta_{0} B-C}{\eta_{0} B+C}\right)^{*}$ represents the complex conjugate of the term $\left(\frac{\eta_{0} B-C}{\eta_{0} B+C}\right)$; similarly, the term $\left(\eta_{0} B+C\right)^{*}$ represents the complex conjugate of the term $\left(\eta_{0} B+C\right)$; and:

$$
\left[\begin{array}{l}
B \\
C
\end{array}\right]=\left(\prod_{r=1}^{p}\left[\begin{array}{cc}
\cos \delta_{r} & \left(j \sin \delta_{r}\right) / \eta_{r} \\
j \eta_{r} \sin \delta_{r} & \cos \delta_{r}
\end{array}\right]\right)\left[\begin{array}{c}
1 \\
\eta_{m}
\end{array}\right]
$$

where $\delta_{r}$ is the phase thickness of the $r$-th layer, having the form of:

$$
\delta_{r}=2 \pi n_{r} d_{r} \cos \theta_{r} / \lambda
$$

where 
$r=$ layer number;

$n_{r}=$ refractive index of the $r$-th layer;

$d_{r}=$ layer thickness of the $r$-th layer;

$\theta_{r}=$ incident angle of the $r$-th layer; and

$\lambda=$ wavelength of the incident radiation.

When a thermal radiation beam is incident onto the coating surface at an incident angle $\theta_{0}$, according to Snell's Law, the beam entering the coatings will transport at a refractive angle $\theta_{r}(r=1,2, \ldots, p)$, and:

$\cos \theta_{r}=\sqrt{1-\left(\frac{n_{0}}{n_{r}}\right)^{2}+\left(\frac{n_{0}}{n_{r}} \cos \theta_{0}\right)^{2}}$

where $n_{0}$ is the refractive index of the incident medium.

Additional symbols in (Eq. 96) are defined as:

$j=$ the symbol of the imaginary component, and $j=\sqrt{-1}$;

$p=$ the total number of layers;

$\eta_{0}, \eta_{r}$ and $\eta_{m}=$ the optical admittances for the incident medium, the particular layer $r$ of interest and the exiting medium of multiple layers, respectively. For oblique incidence, the optical admittance for s-wave (a wave with the direction for electric field normal to the plane of incidence) is expressed as:

$\eta_{z S}=Y n_{z} \cos \left(\theta_{z}\right)$

(Eq. 99)

and the optical admittance for p-wave (a wave with the direction for electric field in the 205 
plane of incidence) is given by:

$\eta_{z P}=\mathrm{Y} n_{z} / \cos \left(\theta_{z}\right)$

(Eq. 100)

where $z=0, r$, or $m$; and $Y=2.6544 \times 10^{-3}$.

If both s-wave and p-wave are incident onto the coating surface from the incident medium, the total reflectance and transmittance of the multiple layered coating can be expressed as:

$\rho_{\lambda}=\frac{1}{2}\left[\rho_{\lambda S}+\rho_{\lambda P}\right]$

(Eq. 101)

$\tau_{\lambda}=\frac{1}{2}\left[\tau_{\lambda S}+\tau_{\lambda P}\right]$

(Eq. 102)

where $\rho_{\lambda S}$ and $\rho_{\lambda P}$ are the reflectance of s-wave and $\mathrm{p}$-wave respectively; and $\tau_{\lambda S}$ and $\tau_{\lambda P}$ are the reflectance of s-wave and p-wave respectively.

The matrix in (Eq. 96) is the characteristic matrix for the multiple layers which provides linkage between various parameters of each layer, such as the layer thickness, refractive index of each layer, and the reflectance and transmittance in the designed TBC structure. Under normal incidence conditions, the reflectance of one stack in air or free space for one wavelength range is expressed as:

$$
\rho_{\lambda} \approx 1-4\left(\frac{n_{L}}{n_{H}}\right)^{2 p} \frac{n_{m}}{n_{H}^{2}}
$$


It can be seen from the above equation that the larger the difference between the highand low- refractive indices, the higher the reflectance; and the more layers within each stack, the higher the reflectance.

\subsubsection{Design of Multiple Layered Stacks}

Based on the fundamental principles described above, a detailed design of the multiple layered coating structures is carried in this section. The targeted wavelength range is $0.3 \sim 5 \mu \mathrm{m}$ due to consideration of the transparency of the selected coating materials. YSZ is selected as the material with higher refractive index values in each stack, and $\mathrm{Al}_{2} \mathrm{O}_{3}$ is selected as the material with lower refractive index values in each stack, and its transmission spectrum is shown in Figure 88.

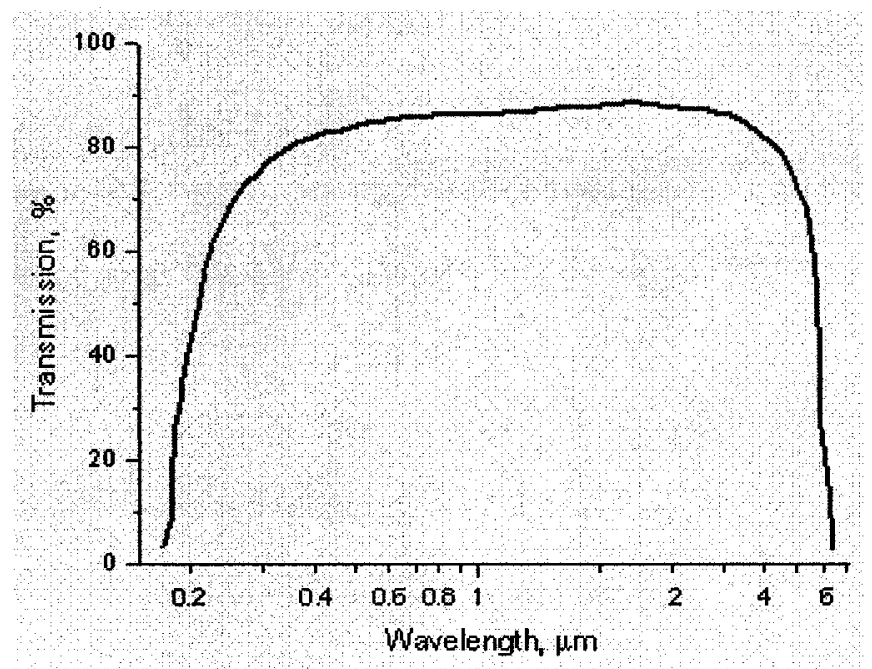

Figure 88 Transmission spectrum of $2 \mathrm{~mm}$-thick sapphire $\left(\mathrm{Al}_{2} \mathrm{O}_{3}\right)$ window [136].

The reason for these selections is based on the availability of physical and thermal properties of both materials and the success in utilizing both materials as commercial TBC. Comparing Figure 29 and Figure 88, it is seen that these two materials have very 
similar transmission spectra, that is, they are fully transparent over a wavelength range of $0.3 \sim 5 \mu \mathrm{m}$ and nearly opaque beyond $5 \mu \mathrm{m}[136]$.

The refractive indices of $\mathrm{YSZ}$ and $\mathrm{Al}_{2} \mathrm{O}_{3}$ are functions of wavelength $\lambda$. The refractive index of 7YSZ can be evaluated by $[137,138]$ :

$n_{7 Y S Z}^{2}-1=\sum_{i=1}^{3} A_{i} \lambda^{2} /\left(\lambda^{2}-L_{i}^{2}\right)$

(Eq. 104)

where $A_{1}=2.118 ; A_{2}=1.347 ; A_{3}=9.453 ;$ and $L_{l}=0.167 ; L_{2}=0.063 ; L_{3}=24.321 ;$ and the refractive index of $\mathrm{Al}_{2} \mathrm{O}_{3}$ can be obtained from [139]:

$n_{A l_{2} \mathrm{O}_{3}}=1.7436-0.02275(\lambda-1.67)$

(Eq. 105)

To obtain high reflectance to thermal radiation in the wavelength range of $0.3 \sim 5 \mu \mathrm{m}$, the $\mathrm{YSZ} / \mathrm{Al}_{2} \mathrm{O}_{3}$ multiple layered coating system is designed with a total of 12 stacks and 12 layers within each stack. This should achieve greater than $80 \%$ hemispherical reflectance. The layer thicknesses for each stack in this design were calculated, and the first and last stacks are described in Table 23. The total physical thickness of the 12 multi- layered stacks is $44.9 \mu \mathrm{m}$.

By integrating (Eq. 101) over the incidence angle $\theta_{0}$ from 0 to $90^{\circ}$, the theoretical hemispherical reflectance of the designed multiple layered stacks is calculated and is shown in Figure 89, where the refractive index of the incident medium is assumed to be 1.0. It can be seen that greater than $80 \%$ hemispherical reflectance can be achieved using 
this design.

Table 23 Physical thickness of each layer for the first and last stacks in designing a multiple layered TBC coating system

\begin{tabular}{|c|c|c|c|c|c|c|}
\hline \multirow{2}{*}{$\begin{array}{l}\text { Stack } \\
\text { number }\end{array}$} & \multirow{2}{*}{$\begin{array}{c}\text { Targeted } \\
\text { wavelength } \\
(\mu \mathrm{m})\end{array}$} & \multirow{2}{*}{$\begin{array}{l}\text { Number } \\
\text { of layers }\end{array}$} & \multicolumn{2}{|c|}{ Refractive index } & \multicolumn{2}{|c|}{$\begin{array}{l}\text { Thickness of each layer } \\
\qquad(\mu \mathrm{m})\end{array}$} \\
\hline & & & High & Low & High index & Low index \\
\hline 1 & 0.5 & 12 & 2.18 & 1.58 & 0.0595 & 0.0823 \\
\hline 12 & 5.57 & 12 & 2.18 & 1.58 & 0.663 & 0.928 \\
\hline
\end{tabular}

Hemispherical Reflectance of 144 Layers

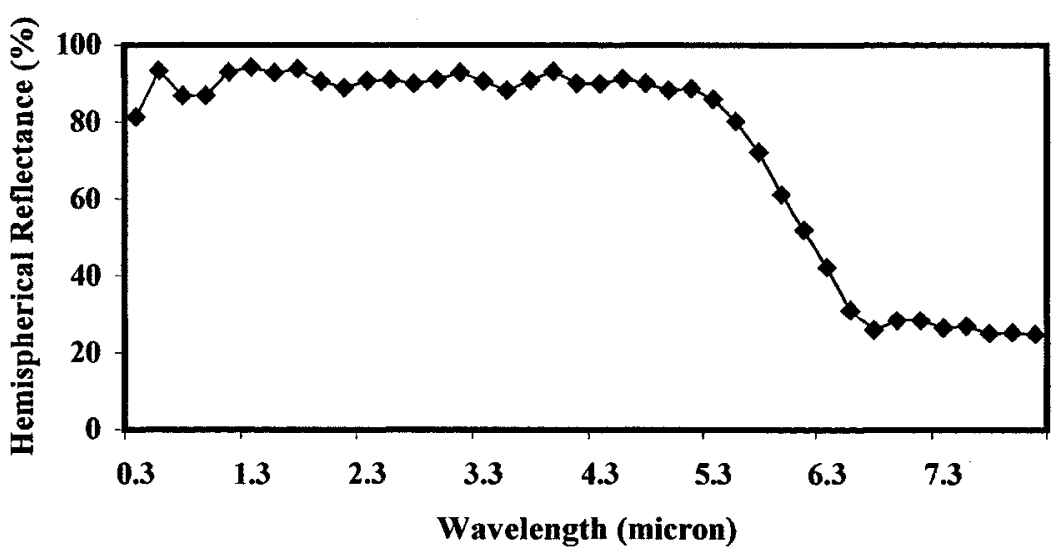

Figure 89 Computed hemispherical reflectance of multiple layered coatings with 12 stacks. 


\subsection{Multiple Layered Coating Structures Incorporating Multiple Layered Stacks}

To effectively reduce heat transfer to the metal substrate, thermal barrier coating systems should be designed to reduce both thermal conduction and thermal radiation. This can be accomplished by means of combining the coating materials with low intrinsic thermal conductivity and incorporating the high reflectance coating structures.

Three different structures consisting of one or two single layers of ceramic material with low thermal conductivity and multiple layered stacks with high reflectance to radiation are considered in this study. Schematic diagrams of the three structures (B, C and D) are presented in Figure 90. Structure A is the baseline mono-layered coating structure of the same thickness. In structure $B$, a single layer $\left(\mathbf{S}_{1}\right)$ with low thermal conductivity is placed under the top layer of multiple layered stacks (M). In structure C, the coating starts with a single layer $\left(\mathbf{S}_{1}\right)$ with low thermal conductivity and low refractive index, beneath which are the multiple layered stacks (M). In structure $\mathrm{D}$, there are two multiple layered stacks $(\mathbf{M})$, and a single layer $\left(\mathbf{S}_{1}\right)$ is placed between these two sets of multiple layered stacks. In structure $\mathrm{B}, \mathrm{C}$ and $\mathrm{D}$, a thin $7 \mathrm{YSZ}$ based ceramic layer $\left(\mathbf{S}_{2}, 5 \mu \mathrm{m}\right)$ is added immediately adjacent to the bond coat to provide thermal stability and bonding to the thermally grown oxide (TGO). This is only needed if material other than 7YSZ is selected for the last layer of either the multiple layered stacks or the single layer. The total thickness for structure A to D is kept to $250 \mu \mathrm{m}$, irrespective of the arrangement of multiple layered stacks; and the thickness of multiple layered stacks is $44.9 \mu \mathrm{m}$ using the 
design described in 7.1 .

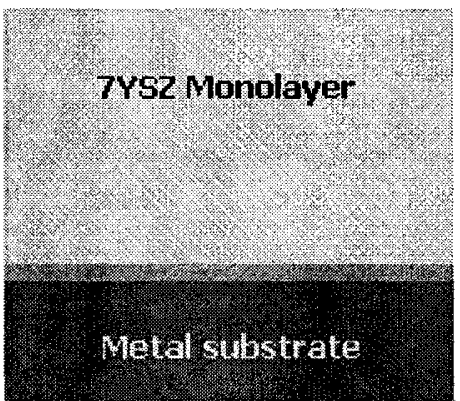

(a) Structure A

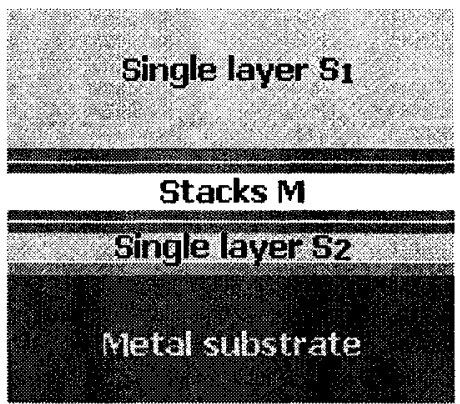

(c) Structure $\mathrm{C}$

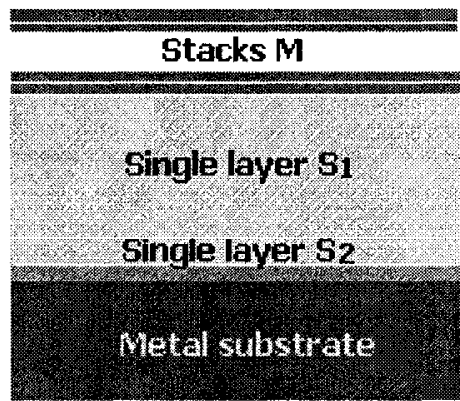

(b) Structure B

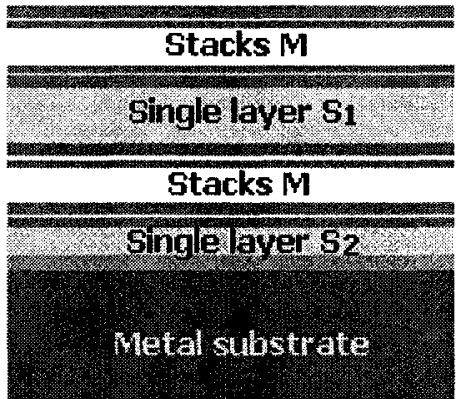

(d) Structure D

Figure 90 Multiple layered coating structures. Structure A: monolayer; structure B: M (top) $+S_{1}+S_{2} ;$ structure $C: S_{1}$ (top) $+M+S_{2} ;$ structure D: $M$ (top) $+S_{1}+M+S_{2}$.

The selection of $\mathbf{S}_{\mathbf{1}}$ single ceramic layer was based on the following considerations. For structures $A$ to $D$, the function of the single layer $S_{1}$ is to reduce thermal conduction through the coatings to the metal substrate. Thus, low thermal conductivity is the basic requirement for this single layer. For example, suitable materials can be either doped 7YSZ, such as $\mathrm{Yb}_{2} \mathrm{O}_{3}$ doped 7YSZ, as detailed in Chapter 6, or zirconates, which have been proven to have low thermal conductivities. The second consideration is that the configuration of the single layer $S_{1}$ should not significantly affect the reflectivity of the multiple layered stacks to thermal radiation. For structure $B$, the single layer $\mathbf{S}_{\mathbf{1}}$ under the 
multiple layered stacks is also required to have high scattering coefficient so that both transmitted and emitted radiation can be scattered away from the metal substrate and at the same time to have high refractive index to increase the reflectivity of the multiple layered stacks. On the other hand, if the single layer is designed to be on top of the multiple layered stacks (Structure C), it is required to have low scattering coefficient and low refractive index. Since the emissive power in a medium is proportional to the square of the refractive index of the material [82], low refractive index of $S_{1}$ layer will ensure reduced internal radiation emitted by $\mathbf{S}_{1}$ layer. Besides, a low refractive index can also reduce internal reflection at the interface between the coating surface and the gas, and result in decreased radiation reflected back into the coatings. Thus, the selection of low refractive index ensures decreased internal reflection at the coating surface and minimum internal radiation emitted in this layer. The selection of low scattering coefficient is to make certain that the reflected radiation from high reflectance multiple layers will not be scattered back. In addition, the single ceramic layer, when placed on the top, is directly exposed to the hot environment, and therefore phase stability is very critical for the performance of TBC system. For example, a zirconate based ceramic material with a pyrochlore structure is potentially the best choice for the top single layer. However, if the single ceramic layer is placed between the multiple layered stacks and the bond coat, zirconates may not be suitable since they are not thermodynamically compatible with TGO. The doped 7YSZ, as examined in Chapter 6, may be inserted as the second thin ceramic single layer $\mathbf{S}_{\mathbf{2}}$. For the same reason, the refractive index of the single layer $\mathbf{S}_{\mathbf{2}}$ material should be as low as possible, whereas the scattering coefficient within the single layer should be high so that the remaining radiation cannot reach the metal substrate. 
However, the selection of materials and the related physical and optical properties have to be optimized systematically in order to minimize the temperatures on both metal and coating surfaces.

To obtain high reflectance, each layer of the multiple layered stacks is required to have a low absorption coefficient and low scattering effect. Thus, extinction is negligible through the medium. As such, the absorption and scattering of radiation within the wavelength range of $0.3 \sim 5 \mu \mathrm{m}$ through the multiple layered stacks are not considered in this study. However, the effect of scattering within the single layer is considered in this study since the majority of the coating thickness is composed of single layer $\mathbf{S}_{1}$.

\subsection{Radiation Transport through the Multiple Layered Coating Structures}

Within the coatings, the radiation wavelength is different from that in vacuum and varies with refractive index of coating materials, whereas the radiation frequency will not change with refractive index. Thus, for convenience of calculation, the radiation frequency, instead of wavelength, will be used in the calculation of the temperature distribution within the multiple layered structures. The $0.3 \sim 5 \mu \mathrm{m}$ wavelength range reflected by the designed multiple layered stacks is equivalent to $10^{15} \mathrm{~Hz} \sim 6 \times 10^{13} \mathrm{~Hz}$. As described in section 7.2 , to achieve high reflectance the absorption and scattering within the multiple layered coatings are required to be as low as possible. Therefore, the absorption and scattering of radiation within the wavelength range of $0.3 \sim 5 \mu \mathrm{m}\left(10^{15} \mathrm{~Hz}\right.$ $\sim 6 \times 10^{13} \mathrm{~Hz}$ ) through the multiple layered stacks are not considered in this study. Under this assumption, if the reflectance of the multiple layered coating is $\rho_{M v}\left(\theta_{0}\right)$, where $\theta_{0}$ is 
the incident angle of radiation onto the surface of multiple layered stacks and $v$ is the radiation frequency, the transmitted radiation intensity through the multiple layers $i_{v T}\left(\theta_{0}\right)$ is:

$i_{v T}\left(\theta_{0}\right)=i_{v i} \cdot\left[1-\rho_{M v}\left(\theta_{0}\right)\right]$

(Eq. 106)

where $i_{v i}$ is the intensity of radiation before entering the multiple layers, and $\theta_{0}$ is the incident angle of radiation onto the surface. Similarly, the reflected intensity from the multiple layers $i_{v R}\left(\theta_{0}\right)$ is given by:

$i_{v R}\left(\theta_{0}\right)=i_{v i} \cdot \rho_{M v}\left(\theta_{0}\right)$

(Eq. 107)

Thus, the spectral radiation flux $q_{r v}$ through the multiple layers is:

$q_{r v T} d v=2 \pi d v\left(\frac{n_{0}}{n_{m}}\right)^{2} \int_{b_{0}=0}^{\pi / 2} i_{v T}\left(\theta_{0}\right) \cos \theta_{0} \sin \theta_{0} d \theta_{0}=2 \pi d v\left(\frac{n_{0}}{n_{m}}\right)^{2} \int_{\mu_{0}=0}^{d} i_{v i}\left[1-\rho_{v}\left(\mu_{0}\right)\right] \mu_{0} d \mu_{0}$

(Eq. 108)

where $\mu_{0}=\cos \theta_{0}$. 


\section{Governing Equations of the Designed TBC Structures}

To analyze the effect of the designed highly reflective multiple layered coating structures on heat transfer to a metal substrate, energy equations of the entire TBCs system should be established and evaluated.
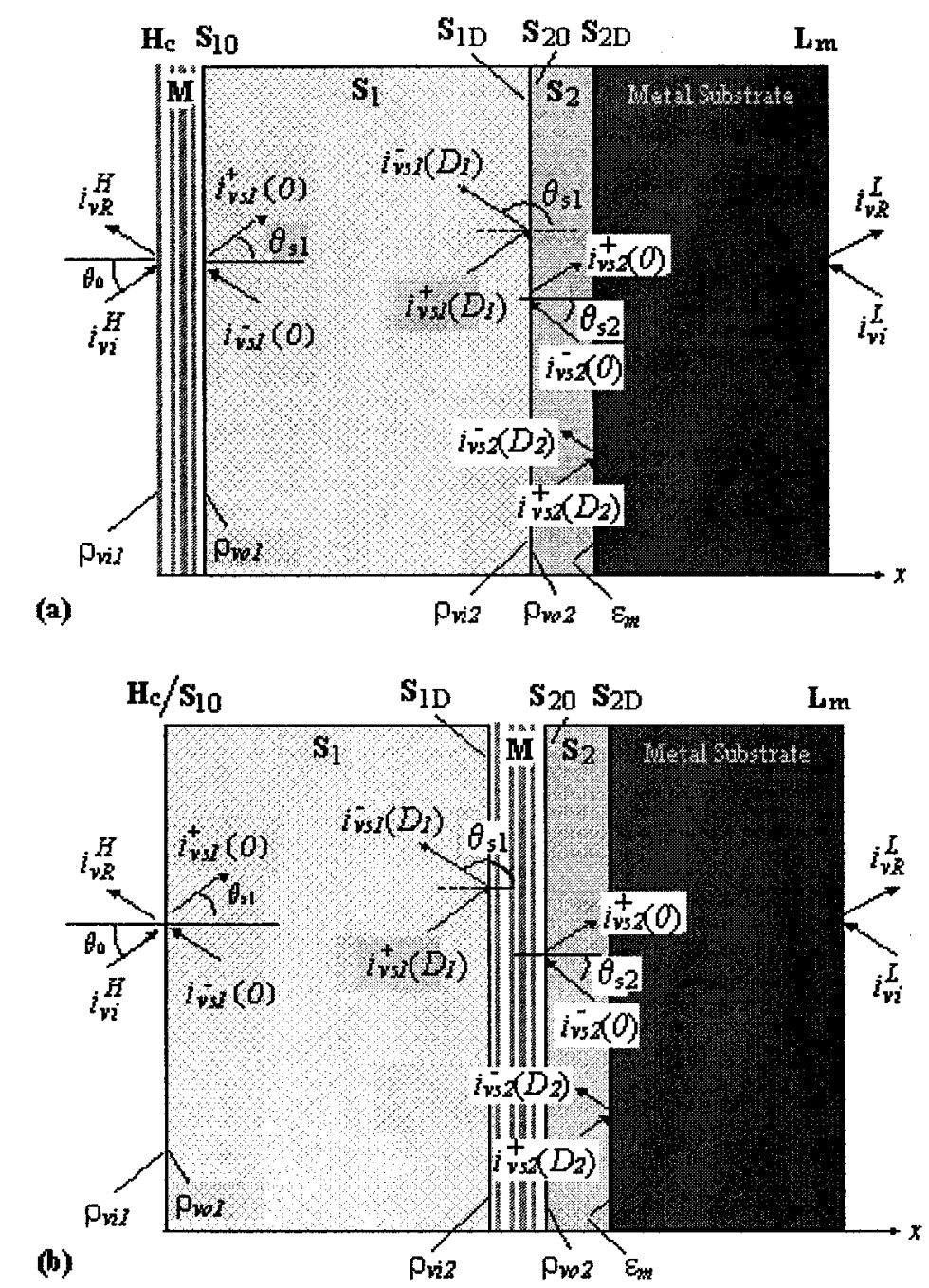

Figure 91 Schematic diagrams of radiation through the high reflectance multiple layered structures: (a) structure B; (b) structure C. 
Figure 91 shows schematics of structures $\mathrm{B}$ and $\mathrm{C}$ to illustrate various boundary conditions for solving both energy equations and related radiation flux equations. As seen, the coating surface Hc is exposed to, and heated by, the hot gases. On the metal surface $\mathbf{L m}$, the heat was taken away by convection and radiation from the hot surface to the cool environment. There is no other heat source inside the system. Assuming the heat transfer has reached a steady state, the total heat flux $Q_{\text {tot }}$ becomes a constant.

\subsection{Energy Equations}

Within coatings, the heat is transported by thermal conduction and thermal radiation. For a one dimensional heat transfer condition, the energy equation can be written as:

$$
k_{l} \frac{d^{2} T_{l}\left(x_{l}\right)}{d x_{l}^{2}}-\frac{d q_{r l}\left(x_{l}\right)}{d x_{l}}=0 \quad l=\left\{\begin{array}{c}
S_{1}, S_{2} \\
1,2, \ldots p
\end{array}\right.
$$

where $S_{I}$ and $S_{2}$ are the subscript indices for single ceramic layer $\left(\mathbf{S}_{1}\right)$ and $7 \mathrm{YSZ}$ thin layer $\left(\mathbf{S}_{2}\right)$, respectively; $p$ is the number of multiple layers; $q_{r l}\left(x_{l}\right)$ is the radiation flux of the $l$-th layer of the coatings in the $x$ direction; $k_{l}$ is thermal conductivity of the $l$-th layer; and $T_{l}\left(x_{l}\right)$ is the temperature at $x$ position of the $l$-th layer of the coating.

Within the metal substrate, the radiation is absorbed and converted into heat; and consequently there is only thermal conduction as a mode of heat transfer. Thus we have:

$$
k_{m} \frac{d^{2} T_{m}\left(x_{m}\right)}{d x_{m}^{2}}=0
$$

where $k_{m}$ is the thermal conductivity of metal substrate and $T_{m}\left(x_{m}\right)$ is the temperature at 216 
position $x_{m}$ within the metal substrate. The metallic bond coat is included as part of the metal substrate in this model.

The integration of the energy equations above gives a constant heat flux $Q_{t o t}$ within the coatings:

$Q_{t o t}=-k_{l} \frac{d T_{l}\left(x_{l}\right)}{d x_{l}}+q_{r l}\left(x_{l}\right)=-k_{m} \frac{d T_{m}\left(x_{m}\right)}{d x_{m}}$

(Eq. 111)

The boundary conditions used to solve energy equations (Eq. 109) and (Eq. 110) are specified as follows. Since the coating surface is heated by convection and thermal radiation emitted by hot gases, the total heat flux entering into the coating should be the sum of the convection and radiation flux transmitted into the coating surface. The boundary condition at the hot surface He can be written as:

$Q_{t o t}=h_{H}\left[T_{H}-T_{1}(0)\right]+q_{r 1}(0)+q_{r 2}(0)$

(Eq. 112)

where $h_{H}$ is the convection coefficient of hot gases; $T_{H}$ and $T_{l}(0)$ are the temperatures of the hot gases and at the coating surface, respectively; $q_{r I}(0)$ is the radiation flux at the coating surface due to the transparency of the coatings; and $q_{r 2}(0)$ is the radiation exchange between the hot gas and the coating at the coating surface due to the absorption and re-emission in the opaque wavelength range. Under the assumption that the coating surface does not influence other surfaces separated by hot gas, $q_{r 2}(0)$ assumes the form:

$q_{r 2}(0)=\int_{0}^{c} \varepsilon_{c v}\left[\frac{2 \pi n_{0}^{2} h v^{3}}{c_{0}^{2}\left(e^{h v / k_{B} T_{t r}}-1\right)}-\frac{2 \pi n_{0}^{2} h v^{3}}{c_{0}^{2}\left(e^{h v / k_{B} T_{i}}-1\right)}\right] d v$

(Eq. 113) 
where $\varepsilon_{c v}$ is the emissivity of the coating material; $v_{c}$ is the cut-off wavelength beyond which the coating is opaque; $n_{0}$ is the refractive index of the hot gases; $T_{i}$ is assumed to be the black body temperature at the hot gas temperature $T_{H}$. And

$h=6.6261 \times 10^{-34} \mathrm{~J} \cdot \mathrm{S}$

$c_{0}=2.99792458 \times 10^{8} \mathrm{~m} / \mathrm{s}$

$k_{B}=1.381 \times 10^{-23} \mathrm{~J} / \mathrm{K}$

Assuming the thickness of the $l$-th coating layer is $\mathrm{D}_{l}$, at the interfaces within coating layers:

$T_{l}\left(D_{l}\right)=T_{l+1}(0)$

where, $l=S_{1}, S_{2}, 1,2, \ldots, p$.

At the cool side of the metal substrate $\mathbf{L m}$, heat is taken away from hot metal due to convection cooling as well as radiation emitted from the hot metal to the cool environment. Thus,

$$
Q_{t o t}=h_{L}\left[T_{m}\left(D_{m}\right)-T_{L}\right]+\varepsilon_{m} \sigma\left[T_{m}^{4}\left(D_{m}\right)-T_{L}^{4}\right]
$$

where $h_{L}$ is the convection coefficient of the cooling fluid; $D_{m}$ is the thickness of the metal substrate, $T_{L}$ and $T_{m}\left(D_{m}\right)$ are the temperatures of the cool environment and metal surface, respectively; $\varepsilon_{m}$ is the emissivity of the metal substrate; and $\sigma$ is the Stefan Boltzmann constant.

The evaluation of boundary conditions for structures $A$ and $D$ is similar to that for structures B and C. 


\subsection{Evaluation of Radiation Flux for Structures A, B, C, and D}

To solve the above energy equations, the evaluation of radiation flux distributions within the ceramic coatings is also required. The radiation flux within both single layers $\mathbf{S}_{1}$ and $S_{2}$ can be obtained by solving the equations of heat transfer from (Eq. 49), (Eq. 50) and (Eq. 51). And the radiation flux through the multiple layered coatings can be obtained from (Eq. 51) and (Eq. 106).

In the computation, the optical properties of both single layers $\left(\mathbf{S}_{\mathbf{1}}\right.$ and $\left.\mathbf{S}_{\mathbf{2}}\right)$ are referred to that of 7YSZ single crystal (Figure 29), and the optical properties of multiple layered stacks are referred to those of both $7 \mathrm{YSZ}$ and $\mathrm{Al}_{2} \mathrm{O}_{3}$ single crystals. Thus, there is no absorption when radiation falls within the wavelength range of $0.3 \sim 5 \mu \mathrm{m}\left(10^{15} \mathrm{~Hz} \sim 6 \mathrm{x}\right.$ $\left.10^{13} \mathrm{~Hz}\right)$; on the other hand, the radiation within the wavelength range of $5 \sim 10 \mu \mathrm{m}(6 \mathrm{x}$ $10^{13} \mathrm{~Hz} \sim 2.31 \times 10^{13} \mathrm{~Hz}$ ) experiences partial absorption and re-emission when transmitting through the YSZ single layers, and total absorption when transmitting through the multiple layered coatings. Beyond $10 \mu \mathrm{m}\left(2.31 \times 10^{13} \mathrm{~Hz} \sim 0\right)$, both single layered coatings and multiple layered coating are opaque and the radiation is totally absorbed.

The evaluation of radiation flux is divided into two parts: radiation flux through the coatings without absorption within the wavelength range of $0.3 \sim 5 \mu \mathrm{m}\left(10^{15} \mathrm{~Hz} \sim 6 \times 10^{13}\right.$ $\mathrm{Hz}$ ) and radiation flux through the coatings with absorption within the wavelength range of $5 \sim 10 \mu \mathrm{m}\left(\sim 6 \times 10^{13} \mathrm{~Hz} \sim 2.31 \times 10^{13} \mathrm{~Hz}\right)$. 


\subsubsection{Radiation Flux within the Wavelength Range of $0.3 \sim 5 \mu \mathrm{m}$}

Due to the transparent nature of ceramics at the wavelength range of $0.3 \sim 5 \mu \mathrm{m}\left(10^{15} \mathrm{~Hz}\right.$ $\sim 6 \times 10^{13} \mathrm{~Hz}$ ), the absorption for both single and multi-layers is considered to be insignificant. For simplicity, the scattering coefficient within multiple layered stacks is assumed to be zero. Thus, the absorption $\alpha_{v}$, albedo $\Omega_{v}$ and optical depth $\kappa_{v}$ within the single layered and multiple layered coatings can be written as:

Within single layers $\mathbf{S}_{\mathbf{1}}$ and $\mathbf{S}_{\mathbf{2}}$ :

$$
\begin{aligned}
& \alpha_{v s}=0 \\
& \Omega_{v s}=1 \\
& \kappa_{v s}(x)=\int_{0}^{x}\left[\alpha_{v s}\left(x^{*}\right)+\sigma_{v s}\left(x^{*}\right)\right] d x^{*}=\int_{0}^{x} \sigma_{v s}\left(x^{*}\right) d x^{*}
\end{aligned}
$$

Within the multiple layered coatings:

$$
\begin{aligned}
& \alpha_{v M}=0 ; \\
& \sigma_{v M}=0 ; \\
& \kappa_{v M}(x)=0 .
\end{aligned}
$$

(Eq. 117)

Under the same conditions, the source function $I_{\mathrm{vs}}\left(\kappa_{v s}, \omega\right)$ in (Eq. 43) can be reduced to:

$$
\begin{aligned}
& I_{v s}\left(\kappa_{v s}, \omega\right)=\frac{1}{4 \pi} \int_{v_{i}=0}^{4 \pi} i_{v s}\left(\kappa_{v s}, \omega_{i}\right) d \omega_{i} \\
& =\frac{1}{2}\left[\int_{\mu_{s}=0}^{4} i_{v s}^{+}\left(0, \mu_{s}\right) e^{-\kappa_{v s}} \mu_{s} d \mu_{s}+\int_{\mu_{s}=0}^{i} i_{v s}^{-}\left(\kappa_{v D s},-\mu_{s}\right) e^{-\left(\kappa_{v D s}-\kappa_{v s}\right)} \mu_{s} d \mu_{s}\right. \\
& \left.+\int_{\kappa_{v s}^{*}=0}^{\kappa_{v D s}} I_{v s}\left(\kappa_{v s}^{*}\right) E_{1}\left(\kappa_{v s}^{*}-\kappa_{v s} \mid\right) d \kappa_{v s}^{*}\right]
\end{aligned}
$$


where:

$$
E_{1}\left(\left|\kappa_{v s}^{*}-\kappa_{v s}\right|\right)=\int_{\mu_{s}=0} \frac{1}{\mu_{s}} e^{-\left(\kappa_{v s}^{*}-\kappa_{v s}\right)} \mu_{s} d \mu_{s}
$$

The boundary conditions for solving equations (Eq. 49) (Eq. 51) and (Eq. 106) for structures $\mathrm{B}$ and $\mathrm{C}$ are given as follows.

For structure B where the high reflectance multiple layered stacks are located on the surface, radiation intensity at the interface $\left(\mathbf{S}_{\mathbf{1 0}}\right)$ between multiple layers $(\mathbf{M})$ and single layer $\left(\mathbf{S}_{1}\right)$ along the positive $x$ direction are the sum of transmitted incident radiation through multiple layers into the single layer $\left(\mathbf{S}_{1}\right)$ and the reflected radiation along the negative direction by the multiple layered stacks (M), as shown in Figure 91 (a). In contrast, for structure $\mathrm{C}$, the single ceramic layer $\left(\mathbf{S}_{1}\right)$ is in direct contact with the hot gases, radiation intensity at the coating surfaces $\left(\mathbf{S}_{\mathbf{1 0}}\right)$ along the $x$ direction is the sum of transmitted incident radiation into the single layer and the reflected radiation by the internal surface of single layer $\left(\mathbf{S}_{1}\right)$, as shown in Figure 91 (b). The radiation intensity $i_{v s 1}^{+}\left(0, \mu_{s 1}\right)$ within the single layer $S_{1}$, leaving the interface $S_{10}$, in the positive $\mathrm{x}$ direction, can therefore be expressed as:

$$
i_{v s 1}^{+}\left(0, \mu_{s 1}\right)=\left(\frac{n_{s 1}}{n_{0}}\right)^{2}\left(1-\rho_{v i 1}\left(\mu_{0}, \mu_{s 1}\right)\right) i_{v i}^{H}+\rho_{v o 1}\left(\mu_{0}, \mu_{s 1}\right) \cdot i_{v s 1}^{-}\left(0,-\mu_{s 1}\right)
$$

where $\mu_{0}=\cos \theta_{0}$, and $\theta_{0}$ is the incident angle onto the coating surface; $\mu_{s 1}=\cos \theta_{s 1}$, 
and $\theta_{s 1}$ is the radiation direction angle within the single layer $S_{1}$ which can be determined using the Snell's law: $n_{0} \sin \theta_{0}=n_{s 1} \sin \theta_{s 1}$, where $n_{s 1}$ and $n_{0}$ are the refractive indices of the single layer $\mathbf{S}_{1}$ and the hot gases, respectively. In (Eq. 120) $i_{v i}^{H}$ is the incident radiation intensity from hot gases at $\mathrm{Hc}$ and can be expressed as: $i_{v i}^{H}=\frac{2 n_{0}^{2} h v^{3}}{c_{0}^{2}\left(e^{h v / k_{B} T_{H}}-1\right)}$

(Eq. 121) $\rho_{v i 1}\left(\mu_{0}, \mu_{s 1}\right)$ and $\rho_{v o 1}\left(\mu_{0}, \mu_{s 1}\right)$ are structure and interface dependent. For structure B, $\rho_{v i 1}\left(\mu_{0}, \mu_{s 1}\right)$ and $\rho_{v o 1}\left(\mu_{0}, \mu_{s 1}\right)$ are the reflectance of multiple layered stacks at the hot coating surface $\mathbf{H c}$ and the coating interface $\mathbf{S}_{\mathbf{1 0}}$, and can be obtained from (Eq. 94), respectively. For structure $\mathrm{C}, \rho_{v i 1}\left(\mu_{0}, \mu_{s 1}\right)$ and $\rho_{v o 1}\left(\mu_{0}, \mu_{s 1}\right)$ can be obtained by:

$\rho_{v i 1}\left(\mu_{0}, \mu_{s 1}\right)=\rho_{v o 1}\left(\mu_{0}, \mu_{s 1}\right)=\frac{1}{2}\left[\frac{\mu_{0} / \mu_{s 1}-n_{0} / n_{s 1}}{\mu_{0} / \mu_{s 1}+n_{0} / n_{s 1}}\right]^{2}+\frac{1}{2}\left[\frac{\mu_{s 1} / \mu_{0}-n_{0} / n_{s 1}}{\mu_{s 1} / \mu_{0}+n_{0} / n_{s 1}}\right]^{2}$

(Eq. 122)

Radiation intensities at the interfaces $\mathbf{S}_{1 \mathrm{D}}$ and $\mathbf{S}_{20}$ for structures $\mathrm{B}$ and $\mathrm{C}$ are given by the following relationships:

$i_{v s 1}^{-}\left(D_{s 1},-\mu_{s 1}\right)=\left(\frac{n_{s 1}}{n_{s 2}}\right)^{2} \cdot\left[1-\rho_{v o 2}\left(\mu_{s 1}, \mu_{s 2}\right)\right] \cdot i_{v s 2}^{-}\left(0,-\mu_{s 2}\right)+\rho_{v i 2}\left(\mu_{s 1}, \mu_{s 2}\right) \cdot i_{v s 1}^{+}\left(D_{s 1}, \mu_{s 1}\right)$

$i_{v s 2}^{+}\left(0, \mu_{s 2}\right)=\left(\frac{n_{s 2}}{n_{s 1}}\right)^{2} \cdot\left[1-\rho_{v i 2}\left(\mu_{s 1}, \mu_{s 2}\right)\right] \cdot i_{v s 1}^{+}\left(D_{s 1}, \mu_{s 1}\right)+\rho_{v o 2}\left(\mu_{s 1}, \mu_{s 2}\right) \cdot i_{v s 2}^{-}\left(0,-\mu_{s 2}\right)$

(Eq. 124) 
where $\mu_{s 2}=\cos \theta_{s 2}$, and $\theta_{s 2}$ is the radiation direction angle within the single layer $\mathbf{S}_{2}$ and can also be determined using the Snell's law: $n_{s 2} \sin \theta_{s 2}=n_{s 1} \sin \theta_{s 1}$. Where $n_{s 2}$ is the refractive index of the single layer $\mathbf{S}_{2}$. For structure $\mathrm{B}, \rho_{v i 2}\left(\mu_{s 1}, \mu_{s 2}\right)$ and $\rho_{v o 2}\left(\mu_{s 1}, \mu_{s 2}\right)$ are the reflectivity at the interface $\mathbf{S}_{1 \mathbf{D}} / \mathbf{S}_{\mathbf{2 0}}$ and can be evaluated using the same method as that of $\rho_{v i 1}\left(\mu_{0}, \mu_{s 1}\right)$ and $\rho_{v o 1}\left(\mu_{0}, \mu_{s 1}\right)$ as in (Eq. 122). For structure C, $\rho_{v i 2}\left(\mu_{s 1}, \mu_{s 2}\right)$ and $\rho_{v o 2}\left(\mu_{s 1}, \mu_{s 2}\right)$ represent the reflectance of multiple layered stacks at interfaces $S_{1 D}$ and $S_{20}$ and can be evaluated using (Eq. 94).

At the interface between the ceramic layer $\mathbf{S}_{\mathbf{2}}$ and the metal substrate, the radiation intensity reflected by the metal substrate with temperature $T_{m}(0)$, for both structures, is expressed as:

$i_{v s 2}^{-}\left(D_{s 2},-\mu_{s 2}\right)=\left(1-\varepsilon_{m}\right) \cdot i_{v s 2}^{+}\left(D_{s 2}, \mu_{s 2}\right)+\varepsilon_{m} \cdot i_{v b}\left[T_{m}(0)\right]$

(Eq. 125)

where $\varepsilon_{m}$ is the diffused emissivity of the metal substrate; and $i_{v b}\left[T_{m}(0)\right]$ is given by: $i_{v b}\left[T_{m}(0)\right]=\frac{2 n_{s 2}^{2} h v^{3}}{c_{0}^{2}\left(e^{h v / k_{B} T_{m}(0)}-1\right)}$

(Eq. 126)

Using a similar method, the boundary conditions for structures A and D can be obtained.

\subsubsection{Radiation Flux within the Wavelength Range of $5 \sim 10 \mu \mathrm{m}$}

Within the wavelength range of $5 \sim 10 \mu \mathrm{m}\left(6 \times 10^{13} \mathrm{~Hz} \sim 2.31 \times 10^{13} \mathrm{~Hz}\right)$, the radiation transmitting through the single layered coatings $\mathbf{S}_{\mathbf{1}}$ and $\mathbf{S}_{\mathbf{2}}$ will experience scattering, 
absorption, and emission. The absorption coefficient for the single layered coating material YSZ is given by the spectrum shown in Figure 29, and can be expressed as:

$$
\alpha_{v}=\left\{\begin{array}{cc}
1 & v \leq 2.31 \times 10^{13} \mathrm{~Hz} \\
1.16-6.94 \times 10^{-15} v & 2.31 \times 10^{13} \mathrm{~Hz}<v \leq 3.75 \times 10^{13} \mathrm{~Hz} \\
2.4-4 \times 10^{-14} v & 3.75 \times 10^{13} \mathrm{~Hz}<v \leq 6 \times 10^{13} \mathrm{~Hz}
\end{array}\right.
$$

(Eq. 127)

When radiation is transmitted into the multiple layered stacks, the radiation within this wavelength range will be totally absorbed since the material $\mathrm{Al}_{2} \mathrm{O}_{3}$ used in the multiple layered coatings is opaque to this wavelength range. Thus, for structures B and D with multiple layered stacks on top, the cut-off frequency $v_{c}$ in (Eq. 113 ) is $6 \times 10^{13} \mathrm{~Hz}$, and the total radiation flux for these two structures is only considered for the frequency range of $10^{15} \mathrm{~Hz} \sim 6 \times 10^{13} \mathrm{~Hz}$. Whereas for structure $\mathrm{C}$, the cut-off frequency $v_{c}$ is $2.31 \times 10^{13}$ $\mathrm{Hz}$ since the single layer $\mathbf{S}_{1}$ is semi-transparent to radiation within the range of $6 \times 10^{13}$ $\mathrm{Hz} \sim 2.31 \times 10^{13} \mathrm{~Hz}$. Within the first single ceramic layer $S_{1}$, the total radiation flux should include two parts: the radiation within the transparent region and radiation within the translucent region. To obtain the radiation flux within the translucent region, the transfer equations for radiation flux and boundary conditions for the transfer equations within the frequency range $10^{15} \mathrm{~Hz} \sim 6 \times 10^{13} \mathrm{~Hz}$ for structure $\mathrm{C}$ should be adjusted as described below.

For structure $\mathrm{C}$, since the absorption coefficient within the single layer is not negligible within the frequency range $10^{15} \mathrm{~Hz} \sim 6 \times 10^{13} \mathrm{~Hz}$, the source function $I_{\mathrm{vs}}\left(\kappa_{v s}, \omega\right)$ within the translucent region in single layer $\mathbf{S}_{\mathbf{1}}$ should be evaluated using (Eq. 43). At the 
coating surface $\mathbf{H c}\left(\mathbf{S}_{10}\right)$ the boundary condition has the same form as (Eq. 120); whereas at the interface $\mathbf{S}_{1 \mathrm{D}}$, the boundary condition is given by:

$i_{v s 1}^{-}\left(D_{s 1},-\mu_{s 1}\right)=\left(1-\varepsilon_{c v}\right) \cdot i_{v s 1}^{+}\left(D_{s}, \mu_{s 1}\right)+\varepsilon_{c v} \cdot i_{v b}\left(T_{1 D}\right)$

(Eq. 128)

where

$i_{v b}\left(T_{1 D}\right)=\frac{2 n_{s 1}^{2} h v^{3}}{c_{0}^{2}\left(e^{h v / k_{B} T_{1 D}}-1\right)}$

(Eq. 129)

Finally, the total radiation flux at each layer can be obtained by integrating the spectral radiation flux over wavelengths from 0.3 to $10 \mu \mathrm{m}\left(2.31 \times 10^{13} \mathrm{~Hz} \sim 10^{15} \mathrm{~Hz}\right)$ :

$$
q_{r l}(x)=\int_{2.31 \times 10^{13}}^{6 \times 10^{13}} q_{r v}(x) d v+\int_{6 \times 10^{13}}^{10^{15}} q_{r v}(x) d v
$$

(Eq. 130)

To compute the temperature distributions for the four structures, various materials properties are required. More specifically, the refractive index for $\mathrm{Al}_{2} \mathrm{O}_{3}$ can be calculated from (Eq. 84) and thermal conductivity is assumed to be $k_{L}=2.4 \mathrm{Wm}^{-1} \mathrm{~K}^{-1}$. The refractive index of 7YSZ can be calculated from (Eq. 83) and thermal conductivity is assumed to be $k_{H}=0.8 \mathrm{Wm}^{-1} \mathrm{~K}^{-1}$. The scattering coefficient for the single layers $\mathbf{S}_{\mathbf{1}}$ and $\mathbf{S}_{\mathbf{2}}$ was varied by assigning numerical values of $1000 \mathrm{~m}^{-1}$ and $10000 \mathrm{~m}^{-1}$ to represent two different scenarios of low and high scattering conditions for 7YSZ. The refractive indices and the spectrum properties are based on values given in the publications [78, $138,139,140,141]$. The numerical values of various properties used in the computation are summarized in Table 24. 
Table 24 Numerical Values and References

\begin{tabular}{|c|c|c|c|}
\hline Symbols & Description & Values & Ref. \\
\hline$T_{H}(\mathrm{~K})$ & Hot gas temperature & 2000 & [141] \\
\hline$T_{L}(\mathrm{~K})$ & Cool side temperature & 800 & [141] \\
\hline$h_{H}\left(\mathrm{~W} \mathrm{~m}^{-2} \mathrm{~K}^{-1}\right)$ & Convection coefficient & 3000 & [141] \\
\hline$h_{L}\left(\mathrm{~W} \mathrm{~m}^{-2} \mathrm{~K}^{-1}\right)$ & Convection coefficient & 3000 & [141] \\
\hline$k_{m}\left(\mathrm{~W} \mathrm{~m}^{-1} \mathrm{~K}^{-1}\right)$ & Thermal conductivity of metal & 33 & [141] \\
\hline$k_{l}\left(\mathrm{~W} \mathrm{~m}^{-1} \mathrm{~K}^{-1}\right)$ & Thermal conductivities of single layer $S_{1}$ and $S_{2}$ & 0.8 & $7 \mathrm{YSZ}$ \\
\hline$k_{H}\left(\mathrm{~W} \mathrm{~m}^{-1} \mathrm{~K}^{-1}\right)$ & $\begin{array}{l}\text { Thermal conductivity of the layer with high } \\
\text { refractive index (7YSZ is used) }\end{array}$ & 0.8 & By design \\
\hline$k_{L}\left(\mathrm{~W} \mathrm{~m}^{-1} \mathrm{~K}^{-1}\right)$ & $\begin{array}{l}\text { Thermal conductivity of the layer with low } \\
\text { refractive index }\left(\mathrm{Al}_{2} \mathrm{O}_{3} \text { is used) }\right.\end{array}$ & 2.4 & By design \\
\hline$D_{\text {total }}(\mathrm{m})$ & Total thickness of ceramics & 0.000250 & By design \\
\hline$D(\mathrm{~m})$ & $\begin{array}{c}\text { Total thickness of high-reflectance multiple } \\
\text { layered stacks } M\end{array}$ & 0.000045 & By design \\
\hline$D_{m}(\mathrm{~m})$ & Thickness of metal substrate & 0.001 & By design \\
\hline$D_{s 2}(\mathrm{~m})$ & Thickness of $7 \mathrm{YSZ}$ thin layer $\mathrm{S}_{2}$ & 0.000005 & By design \\
\hline$n_{0}$ & Refractive index of gas & 1.0 & [141] \\
\hline$n_{H}$ & $\begin{array}{l}\text { High Refractive index of high-reflectance } \\
\text { multiple layered stacks } M\end{array}$ & 2.1 & $7 \mathrm{YSZ}$ \\
\hline$n_{L}$ & $\begin{array}{l}\text { Low refractive index of high-reflectance } \\
\text { multiple layered stacks } M \text { and the top layer } S\end{array}$ & 1.5 & $\mathrm{Al}_{2} \mathrm{O}_{3}$ \\
\hline$n_{S}$ & Refractive index of single layer & 1.5 & By design \\
\hline$\overline{\sigma_{s v}}\left(\mathrm{~m}^{-1}\right)$ & Scattering coefficient of single layer ceramics & $\begin{array}{c}1000 \text { or } \\
10000\end{array}$ & [141] \\
\hline$\alpha_{s v}\left(\mathrm{~m}^{-1}\right)$ & $\begin{array}{l}\text { Absorption coefficient of single layer ceramic } \\
\text { material }\end{array}$ & 30 & [141] \\
\hline$\varepsilon_{b c}$ & Emissivity of bond coat. & 0.5 & [141] \\
\hline$\varepsilon_{3}$ & Emissivity of substrate & 0.7 & [141] \\
\hline$\varepsilon_{c v}$ & Emissivity of coatings & 0.35 & [141] \\
\hline
\end{tabular}


Based on the above mathematical models, boundary conditions and material properties, the resulting temperature distributions for structures A, B, C and D were computed using the program algorithm detailed in Appendix I.

\subsection{Temperature Distributions through the Multi- Layered Coating Systems}

The results obtained from Appendix I for temperatures at both coating and metal surfaces as well as the total heat and radiation fluxes for structures $\mathrm{A}, \mathrm{B}, \mathrm{C}$ and $\mathrm{D}$ are summarized in Table 25. And the temperature distributions are shown in Figure 92 (a) and (b), illustrating two different scattering coefficients within single layer $\mathbf{S}_{1}$ and $\mathbf{S}_{2}$, respectively.

Table 25 Temperatures on coating and metal surfaces, total heat and radiation fluxes through structures A, B, C and D

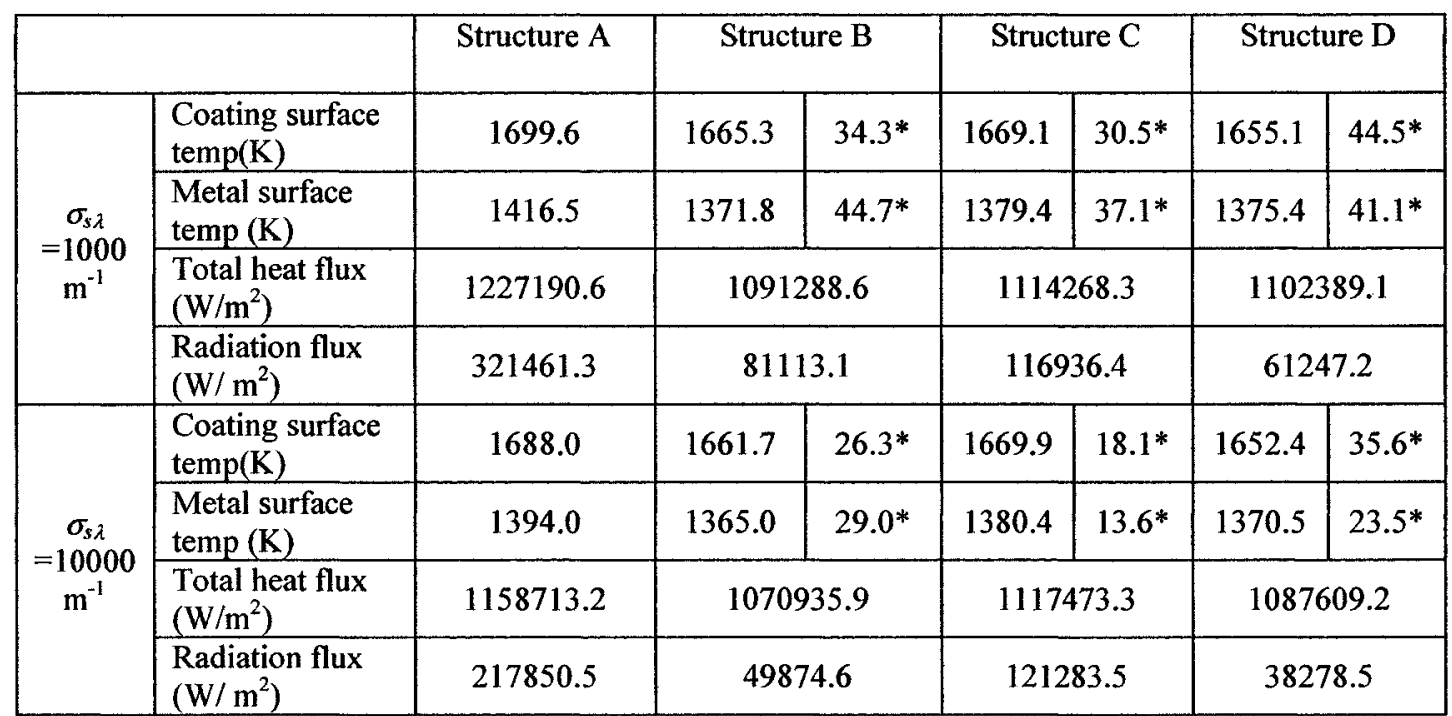

- Temperature differences between structures B, C, D and structure A. 

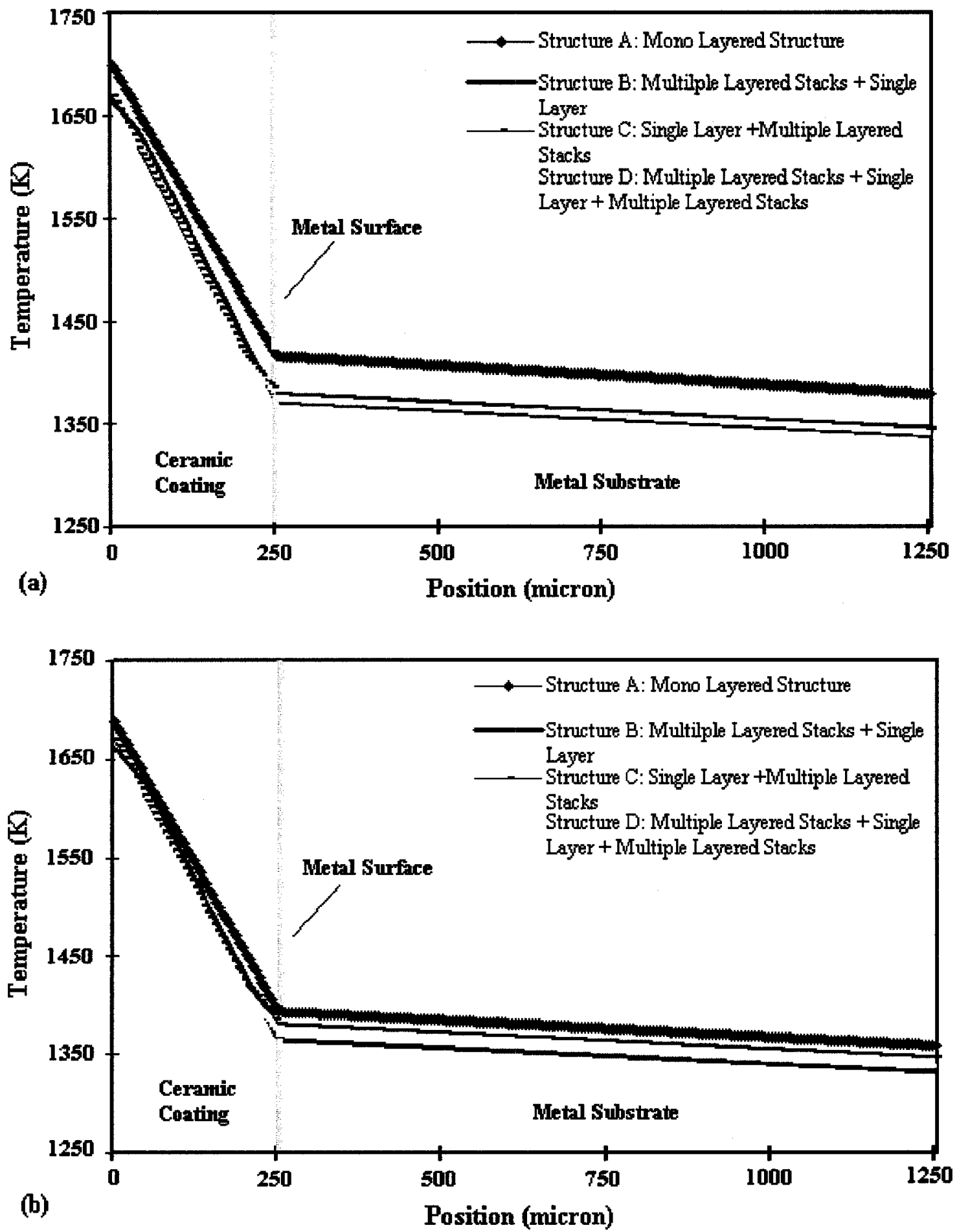

Figure 92 Computed temperature distributions for $250 \mu \mathrm{m}$ thick structures with (a) lower scattering coefficient $\sigma_{\mathrm{s} \lambda}=1000 / \mathrm{m}$; (b) higher scattering coefficient $\sigma_{\mathrm{s} \lambda}=10000 / \mathrm{m}$. 


\subsubsection{Metal Surface Temperatures}

The structures $\mathrm{B}, \mathrm{C}$ and $\mathrm{D}$ exhibit considerable temperature reductions on the metal surface when compared to that of the monolayer coating structure $A$ of the same thickness. Comparing the temperature profiles in Figure 92, it is found that structure B, with multiple layered stacks placed on the top surface, can realize the most significant temperature reduction $\left(44.7^{\circ} \mathrm{C}\right)$ on the metal substrate under a low scattering condition. Surprisingly, structure D with additional multiple stacks adjacent to the metal substrate shows only a $41.1^{\circ} \mathrm{C}$ reduction in temperature on the metal surface. The radiation flux entering structure D is, however, lower than that for structure B. The reason for this anomaly between structures D and B is due to the use of two multiple layered stacks in structure D. This increases the overall thermal conductivity of the structure due to the existence of $\mathrm{Al}_{2} \mathrm{O}_{3}$ in the multiple layered stacks and results in more thermal conduction transport in structure D. If a material with low refractive index and low thermal conductivity could be found to replace $\mathrm{Al}_{2} \mathrm{O}_{3}$, structure $\mathrm{D}$ will provide the most beneficial effect on temperature reduction.

Structure $C$ with multiple layered stacks placed between the single ceramic layer and the metal substrate has a lesser effect on the metal surface temperature reduction when compared with structures B and D. This is due to the use of 7YSZ single layer as the incident medium with a higher refractive index to the multiple layered stacks, which results in a lower hemispherical reflectance when compared to structure $\mathrm{B}$ where the incident medium to the multiple layered stacks is air, with $n_{0}=1.0$. Nevertheless, a $37.1^{\circ} \mathrm{C}$ reduction in temperature on the metal substrate may be achieved. Increasing the 
number of layers in each stack or selecting materials with larger difference in high and low refractive indices could also improve the hemispherical reflectance for structure $\mathrm{C}$ and hence reduce temperature on the metal substrate further.

Thermal barrier coatings deposited by either plasma spray or EB-PVD process could exhibit strong scattering to photons due to certain defects and interfaces in the coating microstructure. Scattering of photons within the coatings redirects radiation transport and can reduce the overall transmitted radiation to the metal substrate. The effect of scattering on heat transport for the four coating structures was investigated by considering low and high scattering coefficients in the computational analysis. Figure 92 (a) and (b) illustrated the calculated temperatures for structures A, B, C, and D with the scattering coefficients assumed to be $1000 \mathrm{~m}^{-1}$ and $10000 \mathrm{~m}^{-1}$, respectively. The metal surface temperatures under various conditions were extracted from Figure 92 (a) and (b) and are plotted in a bar chart as shown in Figure 93. When structure $\mathrm{A}$ is used, the temperature difference on the metal substrate is about $23^{\circ} \mathrm{C}$ when the scattering coefficient is increased from $1000 \mathrm{~m}^{-1}$ to $10000 \mathrm{~m}^{-1}$. For structures $\mathrm{B}$ and $\mathrm{D}$, where the coating surfaces are made of multiple layered stacks, the higher scattering coefficient results in only slight reduction in temperature, approximately $5 \sim 7^{\circ} \mathrm{C}$, on the metal substrate. However, for structure $\mathrm{C}$, with the coating top surface being a single layer $\left(\mathbf{S}_{1}\right)$, an increase in metal surface temperature was found instead when the scattering coefficient is increased from $1000 \mathrm{~m}^{-1}$ to $10000 \mathrm{~m}^{-1}$, as shown in Figure 93. In this structure, since the reflected radiation by the multiple layered stacks is scattered within the top single layer $\left(\mathbf{S}_{1}\right)$, some of the radiation is redirected back to the multiple layered 
stacks and the metal substrate when the scattering coefficient is increased. Consequently, the back reflected radiation is increased accordingly thereby contributing to the increase in the metal surface temperature. Therefore, if structure $\mathrm{C}$ were to be used, the scattering coefficient of the single layer coating should be kept as low as possible.

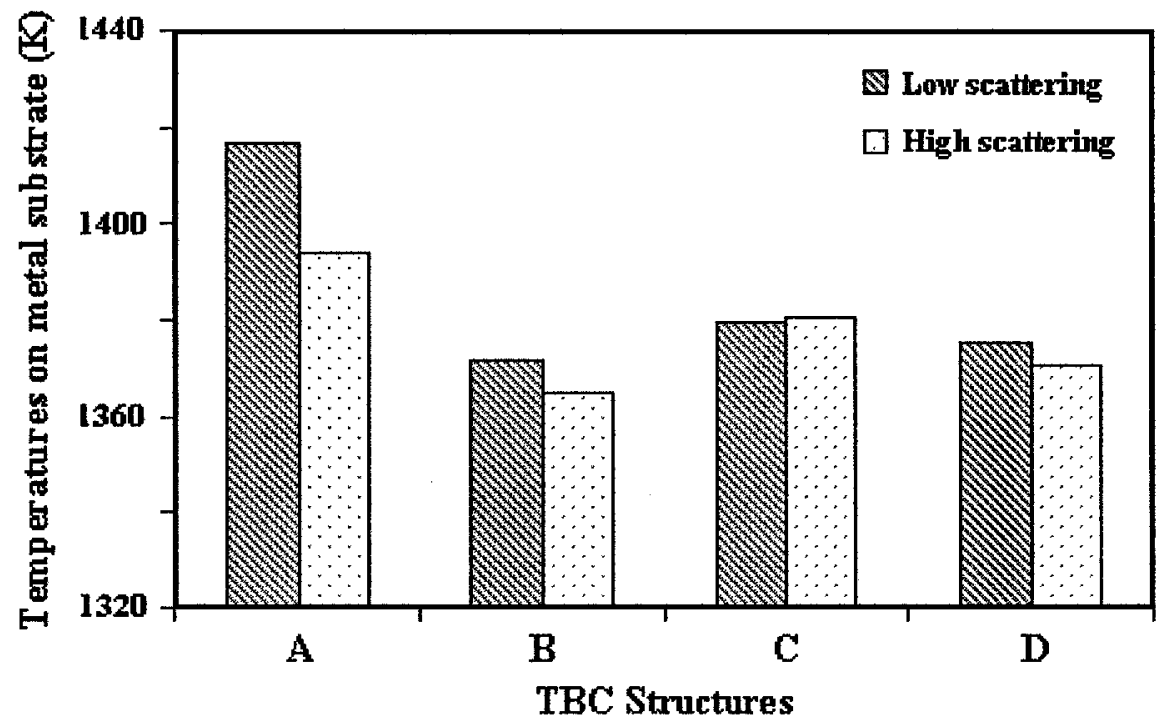

Figure 93 Temperatures on the metal surface for structures A, B, C, and D under two scattering conditions.

\subsubsection{Coating Surface Temperatures}

Comparing the temperature reduction values summarized in Table 25 and Figure 94, it can be seen that in addition to the temperature decrease on the metal surface, the temperatures on the coating surface were significantly reduced as well with the use of structures B, C and D. When multiple layered stacks are present, the radiation flux through the coatings is effectively lowered. Accordingly, the coating surface temperature 
is reduced. In comparison to the coating surface temperature with mono layered coating structure $\mathrm{A}$, structures $\mathrm{B}, \mathrm{C}$ and $\mathrm{D}$ achieved coating surface temperature reductions of $34^{\circ} \mathrm{C}, 31^{\circ} \mathrm{C}$, and $45^{\circ} \mathrm{C}$ respectively with a low scattering coefficient, and $26^{\circ} \mathrm{C}, 18^{\circ} \mathrm{C}$ and $36^{\circ} \mathrm{C}$ respectively with a high scattering coefficient. When the scattering coefficient was increased from $1000 \mathrm{~m}^{-1}$ to $10000 \mathrm{~m}^{-1}$, the absolute temperatures on all coating surfaces, with the exception of structure C, were reduced. Even with the use of mono layered coating structure $\mathrm{A}, \mathrm{a} 12^{\circ} \mathrm{C}$ reduction in temperature was realized when the scattering coefficient was changed from $1000 \mathrm{~m}^{-1}$ to $10000 \mathrm{~m}^{-1}$.

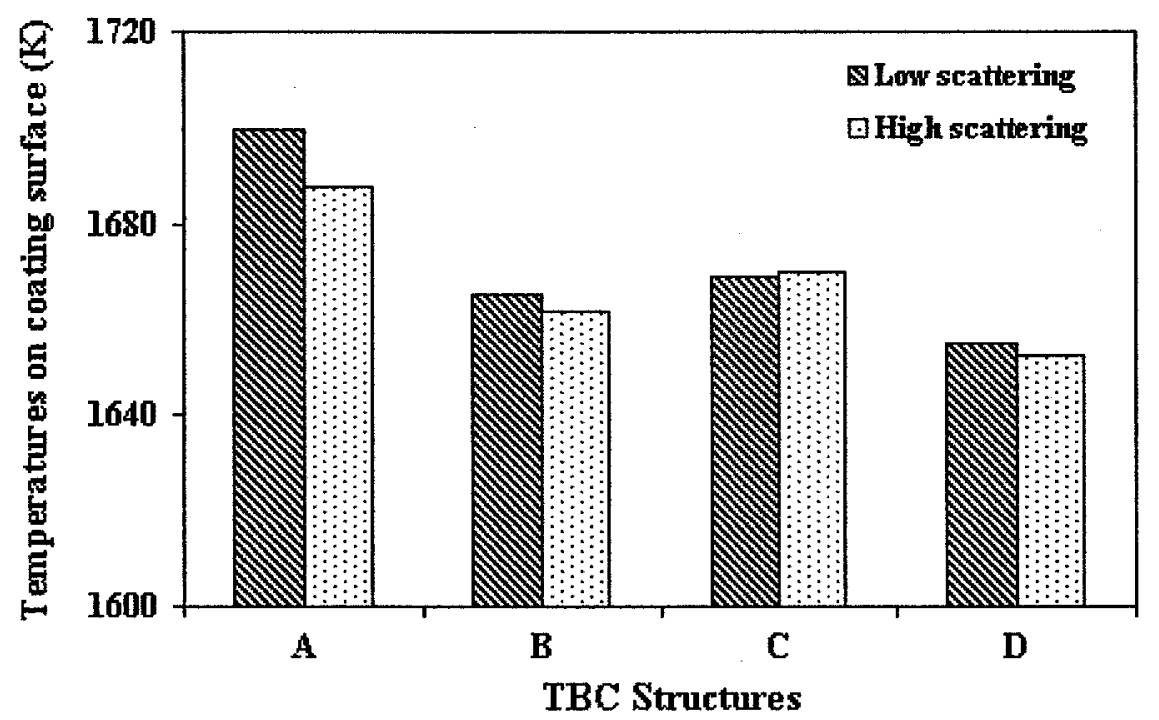

Figure 94 Temperatures on the coating surface for structures A, B, C, and D under two scattering conditions.

\subsubsection{Optimum Multiple Layered Structures}

Under the environmental conditions and material properties assumed in this study, when the multiple layered stacks are located on the coating surface, only the external radiation 
can be effectively reflected and part of the internally emitted radiation will enter the underlying single layered ceramic coating and subsequently into the metal substrate. To effectively reflect both internal and external radiation back into the hot gas stream, it is reasonable to assume that placing the high reflectance multiple layered stacks immediately adjacent to the metal surface (structure C) would produce the best result. However, the results obtained in this study did not agree with this hypothesis. The scattering effect in the single layer was likely the reason behind this. From a structural integrity point of view, it is more desirable to have structure $\mathrm{C}$ since the multiple layered stacks can be well protected by the single top layer so that sintering and interdiffusion can be minimized.

In structure D, where the multiple layered stacks are placed at the coating surface and adjacent to the bond coat, both external and internal radiation can be reflected and therefore structure D has the potential to achieve optimized insulation properties. This, however, could only be realized if a material with low thermal conductivity and low refractive index can be found to replace $\mathrm{Al}_{2} \mathrm{O}_{3}$ in the current design. One may believe that a coating structure containing multiple layered stacks throughout the thickness may be most effective. Preliminary results suggest that a multiple layered coating with repeated spacing (fixed or varied) can effectively achieve increased reflectivity to radiation of one wavelength range, but however, show reduced broad band reflectivity when compared to that of structures $\mathrm{B}, \mathrm{C}$ and $\mathrm{D}$. The physical thicknesses of the high reflectance multiple layered structures in the present design were tailored for different wavelength ranges to reflect broadband thermal radiation, thereby achieving more 
effective thermal radiation reduction. Since the multiple layered structure requires that a minimum of two different materials (with different compositions or varied amounts of defects of the same composition) be used, the thermal conductivity of the coating system comprised of only multiple layers may be compromised as the requirements for maximum difference in refractive indices of the two materials and lowest thermal conductivity for both materials can not always be met. For example, alumina has low refractive index which makes it a good choice to be combined with zirconia to form the high reflectance multiple layered coatings. However, alumina has a higher thermal conductivity and the use of alumina throughout the multiple layered coating will result in increased overall thermal conduction, as demonstrated in comparing structures B and D. The inclusion of a single layer, as shown in structures B, C and D, with low thermal conductivity can compensate for the disadvantage of using alumina and reduce the overall thermal conduction transport since more than $80 \%$ of the coating thickness is comprised of material with low thermal conductivity.

\subsubsection{Novelty of the Current Coating Structures}

Multiple layered coating structures (periodically repeated structure throughout the coatings) have been examined by several researchers [5, 45]. Reduced thermal conductivity as well as high reflectance to thermal radiation at a wavelength of $1.89 \mu \mathrm{m}$ has been reported, as shown in Figure 46. While the metal substrate can be protected when these coatings are applied, the high temperature stability of these multiple layered coatings presents challenges and limitations to their practical use in gas turbine engines. If multiple layers are present on the surface of the coating structure, they will inevitably 
experience the highest temperature of the environment and will be subject to coating failure such as spalling, interdiffusion and other phase transformation and sintering related damages. It is therefore crucial to ensure that the multiple layers used to reflect radiation are shielded from the most severe environment. It is for the first time that the structures B, C, and D combining multiple layered stacks and the single layered coating have been proposed. These designs not only provide high reflectance to the thermal radiation of a broadband wavelength range, but, with careful selection of the coating materials, they also minimize overall thermal conductivity. Additionally, by placing the multiple layered stacks well below the outer surface layer, as in structure $C$, the multiple layered stacks can be better protected. However, this shielding/top layer should be processed to ensure a low scattering nature, otherwise, as shown in this study, the multiple layered structures will not function in an optimum manner.

\subsubsection{Feasibility of the Deposition of the Multiple Layered Coatings}

The designed multiple layered coating structure can be deposited using the EB-PVD process. The multiple layered coatings consist of 12 stacks with each stack having 12 layers. Since the 12 layers within each stack are made of alternating materials and the same material has the same thickness, the coating thickness of each layer can be achieved by controlling the rotational speed $\bar{v}$ of the substrate and by adjusting the shielding angles $\theta_{1}$ and $\theta_{2}$ based on the rates of evaporation of the $\mathrm{Al}_{2} \mathrm{O}_{3}$ and $7 \mathrm{YSZ}$ ingots, as shown in Figure 95 (a). The shield angles $\left(\theta_{1}\right.$ and $\left.\theta_{2}\right)$ determine the thickness ratio between $7 \mathrm{YSZ}$ and $\mathrm{Al}_{2} \mathrm{O}_{3}$; and the rotational speed $\bar{v}$ determines the absolute coating thickness. A thicker coating can be obtained by slower speed, and vice versa. For 
example, the first stack covering the wavelength range of $0.5 \mu \mathrm{m}$ is composed of alternating layers each with thickness of $59.9 \mathrm{~nm}$ for 7YSZ material and $83.3 \mathrm{~nm}$ for $\mathrm{Al}_{2} \mathrm{O}_{3}$ material. If the rates of evaporation of $\mathrm{Al}_{2} \mathrm{O}_{3}$ and $7 \mathrm{YSZ}$ are the same, the ratio of the shielding angles $\theta_{1}$ and $\theta_{2}$ can then be adjusted based on the ratio: $\theta_{1} / \theta_{2}=59.5 / 83.8$. Figure 95 (b) shows the arrangement of a vapor shield for the deposition of the $\mathrm{Al}_{2} \mathrm{O}_{3} / 7 \mathrm{YSZ}$ nanolayer coatings [37].

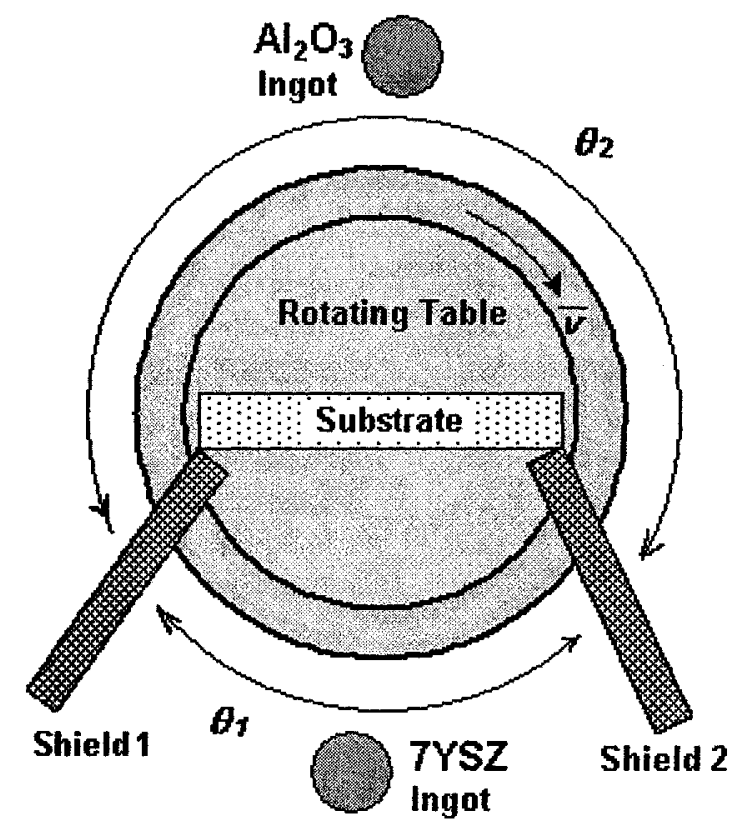

(a)

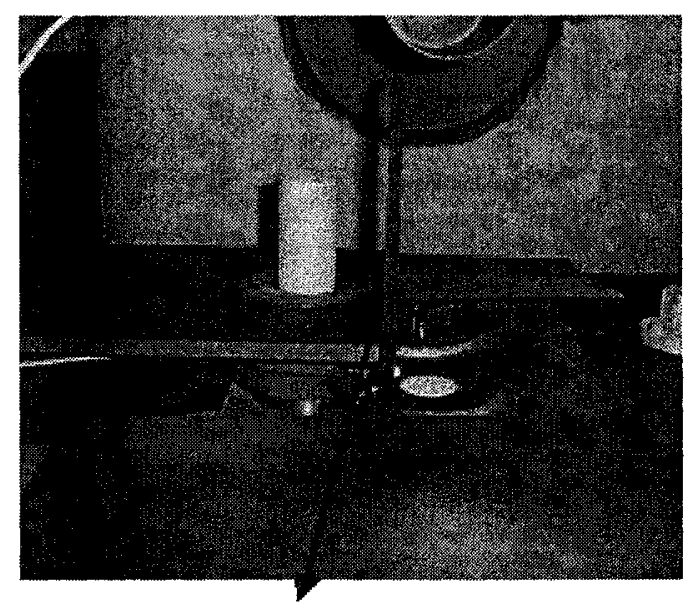

Vapor Shield

(b)

Figure 95 Schematic diagrams of (a) deposition arrangement for obtaining the selected coating thickness of each layer and (b) vapor shield setup for the deposition of the $\mathrm{Al}_{2} \mathrm{O}_{3}$ / 7YSZ nanolayer coatings [37].

After completing each stack, the angle ratio and the rotating speed must be adjusted for the layer thickness of the next stack. However, adjusting these shielding angles during the course of a coating run would not be very practical unless a robot were used. 


\section{Conclusions and Suggested Future Work}

In this study, two approaches were taken to mitigate the heat transfer into TBC systems. First, the doping method was used to study the properties of yttria stabilized zirconia bulk materials doped with various metal oxides such that materials with optimized physical, mechanical and thermal properties could be selected. These were then considered as the target materials for the subsequent multiple layered coatings. Secondly, a multiple layered coating structure was designed to increase the reflectivity of the coating such that the thermal radiation transport through the coating structure could be reduced. Based on the results of this research, the following conclusions can be drawn:

1. Experiments to fabricate bulk zirconia based ceramics by sintering with dopants of pentavalent metal oxides $\left(\mathrm{Ta}_{2} \mathrm{O}_{5}\right.$ and $\left.\mathrm{Nb}_{2} \mathrm{O}_{5}\right)$, trivalent metal oxides $\left(\mathrm{Cr}_{2} \mathrm{O}_{3}, \mathrm{Sc}_{2} \mathrm{O}_{3}\right.$ and $\mathrm{Yb}_{2} \mathrm{O}_{3}$ ), and $\mathrm{CeO}_{2}$ into yttria-stabilized zirconia were conducted. The sintering behaviors of these materials were studied using density, chemical homogeity, and microstructural examinations.

2. The phase compositions of each doped yttria stabilized zirconia material were identified. It was found that the phase stability was determined by the combined effect of both oxygen vacancies and ionic radius of the dopant cations. A dopant cation with the larger ionic radius is the more effective stabilizer.

3. The elastic modulus of each doped yttria stabilized zirconia material was measured. The thermal conductivity of each material was calculated using the results of measured density, specific heat and thermal diffusivity. It was also found that 
5YbYSZ exhibited the lowest elastic modulus and thermal conductivity.

4. A semi-empirical model of thermal conductivities of the doped zirconia based ceramics was established; where it was proposed for the first time that a defect cluster, consisting of substitutional atoms and oxygen vacancies, as one integrated scattering center was responsible for thermal scattering. Conventionally, thermal conductivity models have been based on the atomic defect scattering. The thermal conductivity of each doped material calculated using this model was found to be fully consistent with the experimental results.

5. A multiple layered ceramic coating structure with high reflectance to thermal radiation within the wavelength range of $0.45 \sim 5 \mu \mathrm{m}$ was designed so that the temperature increases caused by thermal radiation on both metal substrate and coating surface are minimized. The hemispherical reflectance of the multiple layered coatings was calculated.

6. Computational analyses to determine temperature profiles for various coating design structures, the influence of scattering, and coating thickness were conducted. From the simulation results, an optimized multiple layered coating structure as well as the key parameters which affect the performance of the structures were discussed.

In light of the results, the following future work is suggested:

1. To optimize the TBC material of each layer to achieve the lowest thermal conductivity and appropriate optical parameters, measurements of refractive index, scattering coefficients and the spectrum of the metal oxide doped yttria stabilized zirconia are required. 
2. Considering the durability of thermal barrier coatings, the thermal expansion coefficient of each doped material is also required.

3. The new multiple layered coating structures should be deposited using the EB-PVD process and the mathematical model validated with the experimental data.

4. The durability characteristics of these new multi-layered TBC systems should be determined under real or simulated gas turbine engine operating conditions.

5. To determined the optical properties of the co-doped materials examined in this study and incorporate the properties values into the multiple layered structures. 


\section{Appendix I Program Algorithm}

- Assume values for $T_{s 1}(0)$ and $T_{s 2}\left(D_{s 2}\right)$.

- Let $\Delta D_{s 1}=\frac{D_{s 1}}{20}$, then for $i$ from 2 to 20 , we have

$$
\begin{aligned}
& x_{i}=(i-1) \cdot \Delta D_{s 1} \\
& T_{s 1}(i)=T_{s 1}(0)+\frac{T_{s 2}\left(D_{s 2}\right)-T_{s 1}(0)}{D_{s 1}+D_{s 2}+D_{M}} \cdot(i-1) \cdot \Delta D_{s 1} \\
& i_{v b 1}\left(x_{i}\right)=\frac{2 n_{s 1}^{2} h v^{3}}{c_{0}^{2}\left(e^{h y / k_{B} T_{s 1}(i)}-1\right)}
\end{aligned}
$$

And

$$
T_{s 2}(0)=T_{s 1}(0)+\frac{T_{s 2}\left(D_{s 2}\right)-T_{s 1}(0)}{D_{s 1}+D_{s 2}+D_{M}} \cdot\left(D_{s 1}+D_{M}\right)
$$

- First, assume that

$$
\begin{aligned}
& I_{v s 1}\left(x_{i}\right)=\left(1-\Omega_{v}\right) \cdot i_{v b 1}\left(x_{i}\right) \\
& \text { For } m=1,2, \cdots 10, \Delta \mu_{s m}=0.02, \text { and } \mu_{s m}=0.8+0.02 \cdot m \text {. Then } \\
& i_{v s 1}^{+}\left(0, \mu_{s m}\right)=\left(\frac{n_{s 1}}{n_{0}}\right)^{2} \cdot\left(1-\rho_{v i 0}\right) \cdot i_{v i}^{H}
\end{aligned}
$$




$$
\begin{aligned}
i_{v s 1}^{+}\left(D_{s 1}, \mu_{s m}\right)= & i_{v s 1}^{+}\left(0, \mu_{s m}\right) \cdot e^{-\mathrm{K}_{v} D_{s 1} \mu_{s m}} \\
& +\frac{\mathrm{K}_{v}}{\mu_{s m}} \cdot \sum_{i=1}^{20}\left[I_{v s 1}\left(x_{i}\right) e^{-\mathrm{K}_{v}\left(D_{s 1}-x_{i}\right)} \mu_{s m}+I_{v s 1}\left(x_{i+1}\right) e^{-\mathrm{K}_{v}\left(D_{s 1}-x_{i+1}\right)} \mu_{s m}\right] \cdot \frac{\Delta D_{s 1}}{2} \\
i_{v s 2}^{+}\left(0, \mu_{s m}\right)= & \left(\frac{n_{s 2}}{n_{s 1}}\right)^{2} \cdot\left[1-\rho_{v M}\left(\mu_{s m}\right)\right] \cdot i_{v s 1}^{+}\left(D_{s 1}, \mu_{s m}\right) \\
i_{v s 2}^{+}\left(D_{s 2}, \mu_{s m}\right)= & i_{v s 2}^{+}\left(0, \mu_{s m}\right) \cdot e^{-\mathrm{K}_{v} D_{s 2}} \mu_{s m}+\frac{\mathrm{K}_{v}}{\mu_{s m}} \cdot\left[I_{v s 2}(0) e^{-\mathrm{K}_{v} D_{s 2} \mu_{s m}}+I_{v s 2}\left(D_{s 2}\right)\right] \cdot \frac{\Delta D_{s 2}}{2}
\end{aligned}
$$

And

$$
\begin{aligned}
& I_{v s 2}(0)=\left(1-\Omega_{v}\right) \cdot i_{v b}\left[T_{s 2}(0)\right] \\
& I_{v s 2}\left(D_{s 2}\right)=\left(1-\Omega_{v}\right) \cdot i_{v b}\left[T_{s 2}\left(D_{s 2}\right)\right]
\end{aligned}
$$

- Evaluate the negative intensities:

$$
\begin{aligned}
& i_{v s 2}^{-}\left(D_{s 2},-\mu_{s m}\right)=\left(1-\varepsilon_{b c}\right) \cdot i_{v s 2}^{+}\left(D_{s 2}, \mu_{s m}\right)+\varepsilon_{b c} \cdot i_{v b}\left[T_{s 2}\left(D_{s 2}\right)\right] \\
& i_{v s 2}^{-}\left(0,-\mu_{s m}\right)=i_{v s 2}^{-}\left(D_{s 2},-\mu_{s m}\right) \cdot e^{-\mathrm{K}_{v} D_{s 2} \mu_{s m}} \\
& +\frac{\mathbf{K}_{v}}{\mu_{s m}} \cdot\left[I_{v s 2}(0)+I_{v s 2}\left(D_{s 2}\right) \cdot e^{-\mathrm{K}_{v} D_{s 2} \mu_{s m}}\right] \cdot \frac{\Delta D_{s 2}}{2} \\
& i_{v s 2}^{-}\left(D_{s 1},-\mu_{s m}\right)=\left(1-\rho_{v M}\left(\mu_{s m}\right) \cdot i_{v s 2}^{-}\left(0,-\mu_{s m}\right)+\rho_{v M}\left(\mu_{s m}\right) \cdot i_{v s 1}^{+}\left(D_{s 1}, \mu_{m}\right)\right.
\end{aligned}
$$




$$
\begin{aligned}
i_{v s 1}^{-}\left(0,-\mu_{s m}\right)= & i_{v s 1}^{-}\left(D_{s 1},-\mu_{s m}\right) \cdot e^{-\mathrm{K}_{v} D_{s 1} / \mu_{s m}} \\
& +\frac{\mathrm{K}_{v}}{\mu_{s m}} \cdot \sum_{i=1}^{20}\left[I_{v s 1}\left(x_{i}\right) e^{-\mathrm{K}_{v} x_{i} / \mu_{s m}}+I_{v s 1}\left(x_{i+1}\right) e^{-\mathrm{K}_{v} x_{i+1} / \mu_{s m}}\right] \cdot \frac{\Delta D_{s 1}}{2}
\end{aligned}
$$

- Re-evaluate valuate source function and radiation intensities.

For $j=2, \cdots n$,

$$
\begin{aligned}
& I_{v s 1}\left(x_{i}\right)=\left(1-\Omega_{v}\right) \cdot i_{v b 1}\left(x_{i}\right) \\
& +\frac{\Omega_{v}}{2}\left\{\sum_{m=1}^{9}\left[i_{v s 1}^{+}\left(0, \mu_{s m}\right) \cdot e^{-\mathrm{K}_{v} x_{i} / \mu_{s m}}+i_{v s 1}^{+}\left(0, \mu_{s m+1}\right) \cdot e^{-\mathrm{K}_{v} x_{i} / \mu_{s m+1}}\right] \cdot \frac{\Delta \mu_{s m}}{2}\right. \\
& +\sum_{m=1}^{9}\left[i_{v s 1}^{-}\left(D_{s 1},-\mu_{s m}\right) \cdot e^{-\mathrm{K}_{v}\left(D_{s 1}-x_{i}\right) / \mu_{s m}}+i_{v s 1}^{-}\left(D_{s 1},-\mu_{s m+1}\right) \cdot e^{-\mathrm{K}_{v}\left(D_{s 1}-x_{i}\right) / \mu_{s m+1}}\right] \cdot \frac{\Delta \mu_{s m}}{2} \\
& \left.+\mathrm{K}_{v} \frac{\Delta D_{s 1}}{2} \sum_{k=1}^{20}\left[I_{v s 1}\left(x_{k}\right) E_{1}\left(\mathrm{~K}_{v}\left|x_{k}-x_{i}\right|\right)+I_{v s 1}\left(x_{k+1}\right) E_{1}\left(\mathrm{~K}_{v}\left|x_{k+1}-x_{i}\right|\right)\right]\right\} \\
& I_{v s 2}(0)=\left(1-\Omega_{v}\right) \cdot i_{v b 2}\left[\left(T_{s 2}(0)\right]\right. \\
& +\frac{\Omega_{v}}{2}\left\{\sum_{m=1}^{9}\left[i_{v s 2}^{+}\left(0, \mu_{s m}\right)+i_{v s 2}^{+}\left(0, \mu_{s m+1}\right)\right] \cdot \frac{\Delta \mu_{s m}}{2}\right. \\
& +\sum_{m=1}^{9}\left[i_{v s 2}^{-}\left(D_{s 2},-\mu_{s m}\right) \cdot e^{-\mathrm{K}_{v} D_{s 2} / \mu_{s m}}+i_{v s 2}^{-}\left(D_{s 2},-\mu_{s m+1}\right) \cdot e^{-\mathrm{K}_{v} D_{s 2} / \mu_{s m+1}}\right] \cdot \frac{\Delta \mu_{s m}}{2} \\
& \left.+\mathrm{K}_{v} \frac{\Delta D_{s 2}}{2}\left[I_{v s 2}(0)+I_{v s 2}\left(D_{s 2}\right) E_{1}\left(\mathrm{~K}_{v} D_{s 2}\right)\right]\right\}
\end{aligned}
$$




$$
\begin{aligned}
I_{v s 2}\left(D_{s 2}\right)= & \left(1-\Omega_{v}\right) \cdot i_{v b 2}\left[T_{s 2}\left(D_{s 2}\right)\right] \\
& +\frac{\Omega_{v}}{2}\left\{\sum _ { m = 1 } ^ { 9 } \left[i_{v s 2}^{+}\left(0, \mu_{s m}\right) \cdot e^{-\mathrm{K}_{v} D_{s 2} / \mu_{s m}}+i_{v s 2}^{+}\left(0, \mu_{s m+1}\right) \cdot e^{\left.-\mathrm{K}_{v} D_{s 2} / \mu_{s m+1}\right] \cdot \frac{\Delta \mu_{s m}}{2}}\right.\right. \\
& +\sum_{m=1}^{9}\left[i_{v s 2}^{-}\left(D_{s 2},-\mu_{s m}\right)+i_{v s 2}^{-}\left(D_{s 2},-\mu_{s m+1}\right)\right] \cdot \frac{\Delta \mu_{s m}}{2} \\
& +\mathrm{K}_{v} \frac{\Delta D_{s 2}}{2}\left[I_{v s 2}(0) E_{1}\left(\mathrm{~K}_{v} D_{s 2}\right)+I_{v s 2}\left(D_{s 2}\right)\right]
\end{aligned}
$$

And

$$
\begin{aligned}
i_{v s 1}^{+}\left(0, \mu_{s m}\right)= & \left(\frac{n_{s 1}}{n_{0}}\right)^{2} \cdot\left(1-\rho_{v i 0}\right) \cdot i_{v i}^{H}+\rho_{v s 0} \cdot i_{v s 1}^{-}\left(0,-\mu_{s m}\right) \\
i_{v s 1}^{+}\left(D_{s 1}, \mu_{s m}\right)= & i_{v s 1}^{+}\left(0, \mu_{s m}\right) \cdot e^{-\mathrm{K}_{v} D_{s 1} / \mu_{s m}} \\
& +\frac{\mathrm{K}_{v}}{\mu_{s m}} \cdot \sum_{i=1}^{20}\left[I_{v s 1}\left(x_{i}\right) e^{-\mathrm{K}_{v}\left(D_{s 1} x_{i}\right) / \mu_{s m}}+I_{v s 1}\left(x_{i+1}\right) e^{-\mathrm{K}_{v}\left(D_{s 1}-x_{i+1}\right) / \mu_{s m}}\right] \cdot \frac{\Delta D_{s 1}}{2} \\
i_{v s 2}^{+}\left(0, \mu_{s m}\right)= & \left(\frac{n_{s 2}}{n_{s 1}}\right)^{2} \cdot\left[1-\rho_{v M}\left(\mu_{s m}\right)\right] \cdot i_{v s 1}^{+}\left(D_{s 1}, \mu_{s m}\right)+\rho_{v M}\left(\mu_{s m}\right) \cdot i_{v s 2}^{-}\left(0,-\mu_{s m}\right) \\
i_{v s 2}^{+}\left(D_{s 2}, \mu_{s m}\right)= & i_{v s 2}^{+}\left(0, \mu_{s m}\right) \cdot e^{-\mathrm{K}_{v} D_{s 2} / \mu_{s m}} \\
& +\frac{\mathrm{K}_{v}}{\mu_{s m}} \cdot\left[I_{v s 2}(0) e^{\left.-\mathrm{K}_{v} D_{s 2} / \mu_{s m}+I_{v s 2}\left(D_{s 2}\right)\right] \cdot \frac{\Delta D_{s 2}}{2}}\right.
\end{aligned}
$$

Then 


$$
\begin{aligned}
i_{v s 2}^{-}\left(D_{s 2},-\mu_{s m}\right) & =\left(1-\varepsilon_{b c}\right) \cdot i_{v s 2}^{+}\left(D_{s 2}, \mu_{s m}\right)+\varepsilon_{b c} \cdot i_{v b}\left[T_{s 2}\left(D_{s 2}\right)\right] \\
i_{v s 2}^{-}\left(0,-\mu_{s m}\right)= & i_{v s 2}^{-}\left(D_{s 2},-\mu_{s m}\right) \cdot e^{-\mathrm{K}_{v} D_{s 2} / \mu_{s m}} \\
& +\frac{\mathrm{K}_{v}}{\mu_{s m}} \cdot\left[I_{v s 2}(0)+I_{v s 2}\left(D_{s 2}\right) \cdot e^{-\mathrm{K}_{v} D_{s 2} / \mu_{s m}}\right] \cdot \frac{\Delta D_{s 2}}{2} \\
i_{v s 2}^{-}\left(D_{s 1},-\mu_{s m}\right) & =\left(1-\rho_{v M}\left(\mu_{s m}\right) \cdot i_{v s 2}^{-}\left(0,-\mu_{s m}\right)+\rho_{v M}\left(\mu_{s m}\right) \cdot i_{v s 1}^{+}\left(D_{s 1}, \mu_{m}\right)\right. \\
i_{v s 1}^{-}\left(0,-\mu_{s m}\right)= & i_{v s 1}^{-}\left(D_{s 1},-\mu_{s m}\right) \cdot e^{-\mathrm{K}_{v} D_{s 1} / \mu_{s m}} \\
& +\frac{\mathrm{K}_{v}}{\mu_{s m}} \cdot \sum_{i=1}^{20}\left[I_{v s 1}\left(x_{i}\right) e^{-\mathrm{K}_{v} x_{i} / \mu_{s m}}+I_{v s 1}\left(x_{i+1}\right) e^{-\mathrm{K}_{v} x_{i+1} / \mu_{s m}}\right] \cdot \frac{\Delta D_{s 1}}{2}
\end{aligned}
$$

- Evaluate spectral radiation flux within single layer $\mathrm{S}_{1}$ :

For $i=1$,

$$
\begin{aligned}
q_{v s 1}\left(x_{1}\right)= & 2 \pi \cdot \sum_{m=1}^{9}\left[i_{v s 1}^{+}\left(0, \mu_{s m}\right) \cdot \mu_{s m}+i_{v s 1}^{+}\left(0, \mu_{s m+1}\right) \cdot \mu_{s m+1}\right] \cdot \frac{\Delta \mu_{s m}}{2} \\
& -\pi \cdot \Delta \mu_{s m} \cdot \\
& \sum_{m=1}^{9}\left[i_{v s 1}^{-}\left(D_{s 1},-\mu_{s m}\right) \cdot e^{-\mathrm{K}_{v} D_{s 1}} \mu_{s m} \cdot \mu_{s m}+i_{v s 1}^{-}\left(D_{s 1},-\mu_{s m+1}\right) \cdot e^{-\mathrm{K}_{v} D_{s 1} \mu_{s m+1}} \cdot \mu_{s m+1}\right] \\
& -2 \pi \mathrm{K}_{v} \sum_{k=1}^{20}\left[I_{v s 1}\left(x_{k}\right) E_{2}\left(\mathrm{~K}_{v} x_{k}\right)+I_{v s 1}\left(x_{k+1}\right) E_{2}\left(\mathrm{~K}_{v} x_{k+1}\right)\right] \cdot \frac{\Delta D_{s 1}}{2}
\end{aligned}
$$

For $i=2, \cdots, 20$, 


$$
\begin{aligned}
q_{v s 1}\left(x_{i}\right)= & 2 \pi \cdot \frac{\Delta \mu_{s m}}{2} \cdot \sum_{m=1}^{g}\left[i_{v s 1}^{+}\left(0, \mu_{s m}\right) \cdot \mu_{s m} \cdot e^{-\mathrm{K}_{v} x_{i} / \mu_{s m}}+i_{v s 1}^{+}\left(0, \mu_{s m+1}\right) \cdot \mu_{s m+1} \cdot e^{-\mathrm{K}_{v} x_{i} / \mu_{s m+1}}\right] \\
& -2 \pi \cdot \frac{\Delta \mu_{s m}}{2} \cdot \\
& \sum_{m=1}^{9}\left[i_{v s 1}^{-}\left(D_{s 1},-\mu_{s m}\right) \cdot e^{-\mathrm{K}_{v}\left(D_{s 1}-x_{i}\right) / \mu_{s m}} \cdot \mu_{s m}+i_{v s 1}^{-}\left(D_{s 1},-\mu_{s m+1}\right) \cdot e^{-\mathrm{K}_{v}\left(D_{s 1}-x_{i}\right) / \mu_{s m+1}} \cdot \mu_{s m+1}\right] \\
& -2 \pi \mathrm{K}_{v} \cdot \frac{\Delta D_{s 1}}{2} \sum_{k=i}^{20}\left[I_{v s 1}\left(x_{k}\right) E_{2}\left[\mathrm{~K}_{v}\left(x_{k}-x_{i}\right)\right]+I_{v s 1}\left(x_{k+1}\right) E_{2}\left[\mathrm{~K}_{v}\left(x_{k+1}-x_{i}\right)\right]\right] \\
& +2 \pi \mathrm{K}_{v} \cdot \frac{\Delta D_{s 1}}{2} \sum_{k=1}^{i-1}\left[I_{v s 1}\left(x_{k}\right) E_{2}\left[\mathrm{~K}_{v}\left(x_{i}-x_{k}\right)\right]+I_{v s 1}\left(x_{k+1}\right) E_{2}\left[\mathrm{~K}_{v}\left(x_{i}-x_{k+1}\right)\right]\right]
\end{aligned}
$$

For $i=21$,

$$
\begin{aligned}
q_{v s 1}\left(x_{21}\right)= & 2 \pi \cdot \frac{\Delta \mu_{s m}}{2} \cdot \\
& \sum_{m=1}^{9}\left[i_{v s 1}^{+}\left(0, \mu_{s m}\right) \cdot \mu_{s m} \cdot e^{-\mathrm{K}_{v} D_{s 1} / \mu_{s m}}+i_{v s 1}^{+}\left(0, \mu_{s m+1}\right) \cdot \mu_{s m+1} \cdot e^{\left.-\mathrm{K}_{v} D_{s 1} / \mu_{s m+1}\right]}\right. \\
& -2 \pi \cdot \sum_{m=1}^{9}\left[i_{v s 1}^{-}\left(D_{s 1},-\mu_{s m}\right) \cdot \mu_{s m}+i_{v s 1}^{-}\left(D_{s 1},-\mu_{s m+1}\right) \cdot \mu_{s m+1}\right] \cdot \frac{\Delta \mu_{s m}}{2} \\
& +2 \pi \mathrm{K}_{v} \cdot \frac{\Delta D_{s 1}}{2} \cdot \\
& \sum_{k=1}^{20}\left[I_{v s 1}\left(x_{k}\right) E_{2}\left[\mathrm{~K}_{v}\left(D_{s 1}-x_{k}\right)\right]+I_{v s 1}\left(x_{k+1}\right) E_{2}\left[\mathrm{~K}_{v}\left(D_{s 1}-x_{k+1}\right)\right]\right]
\end{aligned}
$$

Similarly, within the single layer $S_{2}$ : 


$$
\begin{aligned}
q_{v s 2}(0)= & 2 \pi \cdot \sum_{m=1}^{9}\left[i_{v s 2}^{+}\left(0, \mu_{s m}\right) \cdot \mu_{s m}+i_{v s 2}^{+}\left(0, \mu_{s m+1}\right) \cdot \mu_{s m+1}\right] \cdot \frac{\Delta \mu_{s m}}{2} \\
& -2 \pi \cdot \frac{\Delta \mu_{s m}}{2} . \\
& \sum_{m=1}^{9}\left[i_{v s 2}^{-}\left(D_{s 2},-\mu_{s m}\right) \cdot e^{-\mathrm{K}_{v} D_{s 2} / \mu_{s m}} \cdot \mu_{s m}+i_{v s 2}^{-}\left(D_{s 2},-\mu_{s m+1}\right) \cdot e^{-\mathrm{K}_{v} D_{s 2} / \mu_{s m+1}} \cdot \mu_{s m+1}\right] \\
& -2 \pi \mathrm{K}_{v}\left[I_{v s 2}(0)+I_{v s 1}\left(D_{s 2}\right) E_{2}\left(\mathrm{~K}_{v} D_{s 2}\right)\right] \cdot \frac{\Delta D_{s 2}}{2}
\end{aligned}
$$

And

$$
\begin{aligned}
q_{v s 2}\left(D_{s 2}\right)= & 2 \pi \cdot \frac{\Delta \mu_{s m}}{2} \cdot \\
& \sum_{m=1}^{9}\left[i_{v s 2}^{+}\left(0, \mu_{s m}\right) \cdot e^{-\mathrm{K}_{v} D_{s 2} / \mu_{s m}} \cdot \mu_{s m}+i_{v s 2}^{+}\left(0, \mu_{s m+1}\right) \cdot e^{-\mathrm{K}_{v} D_{s 2} / \mu_{s m+1}} \cdot \mu_{s m+1}\right] \\
& -2 \pi \cdot \sum_{m=1}^{9}\left[i_{v s 2}^{-}\left(D_{s 2},-\mu_{s m}\right) \cdot \mu_{s m}+i_{v s 2}^{-}\left(D_{s 2},-\mu_{s m+1}\right) \cdot \mu_{s m+1}\right] \cdot \frac{\Delta \mu_{s m}}{2} \\
& -2 \pi \mathrm{K}_{v}\left[I_{v s 2}\left(D_{s 2}\right)+I_{v s 2}(0) E_{2}\left(\mathrm{~K}_{v} D_{s 2}\right)\right] \cdot \frac{\Delta D_{s 2}}{2}
\end{aligned}
$$

- Evaluate the total radiation flux by integrating the spectral flux over frequency within single layer $S_{1}$ and $S_{2}$, respectively:

$$
\begin{aligned}
& q_{r 1}\left(x_{i}\right)=\int_{2.31 \times 10^{13}}^{10^{15}} q_{r v 1}\left(x_{i}, v\right) d v \\
& q_{r 2}(x)=\int_{b \times 10^{13}}^{10^{15}} q_{r v 2}(x, v) d v
\end{aligned}
$$


- Solve governing equations:

$Q_{t o t}=h_{H}\left[T_{H}-T_{s 1}(0)\right]+q_{r 1}\left(x_{1}\right)+\pi \cdot \int_{l=0}^{2.31 \times 10^{13}} \varepsilon_{c v}\left[i_{v i}-i_{v b}\left(T_{s 1}(0)\right)\right] d v$

From

$Q_{t o t}=h_{L}\left[T_{m}\left(D_{m}\right)-T_{L}\right]+\varepsilon_{m} \sigma\left[T_{m}^{4}\left(D_{m}\right)-T_{L}^{4}\right]$

$T_{m}\left(D_{m}\right)$ can be obtained. And

$T_{m}(0)=T_{s 2}\left(D_{s 2}\right)=T_{m}\left(D_{m}\right)+\frac{Q_{t o t} D_{m}}{k_{m}}$

$T_{s 2}(0)=T_{s 2}\left(D_{s 2}\right)+\frac{Q_{t o t} D_{s 2}}{k_{s 2}}-\frac{1}{k_{s 2}} \frac{D_{s 2}}{2}\left[q_{r 2}(0)+q_{r 2}\left(D_{s 2}\right)\right]$

Assume radiation flux with the multiple layered stacks is

$\bar{q}_{r M} \approx \frac{1}{2}\left[q_{r 1}\left(D_{s 1}\right)+q_{r 2}(0)\right]$

Then

$$
\begin{aligned}
& T_{s 1}\left(D_{s 1}\right)=T_{s 2}(0)+\left(\frac{\sum_{n=1}^{72} D_{2 n}}{k_{L}}+\frac{\sum_{n=1}^{72} D_{2 n-1}}{k_{H}}\right)\left(Q_{t o t}-\bar{q}_{r M}\right) \\
& T_{s 1}(0)=T_{s 1}\left(D_{s 1}\right)+\frac{Q_{t o t} D_{s 1}}{k_{s 1}}-\frac{1}{k_{s 1}} \frac{\Delta D_{s 1}}{2}\left[q_{r 1}\left(x_{i}\right)+q_{r 1}\left(x_{i+1}\right)\right]
\end{aligned}
$$


Evaluate temperature distribution at each layer within the multiple stacks:

$$
T_{l}\left(x_{l}\right)=T_{l}(0)-\frac{Q_{t o t}}{k_{l}} x_{l}+\frac{x_{l}}{2 k_{l}}\left[q_{r l}(0)+q_{r l}\left(x_{l}\right)\right] \quad l=1,2, \cdots, 144
$$

where the variation of $q_{r l}\left(x_{l}\right)$ within one thin layer is very small, thus

$$
\int_{b}^{x_{l}} q_{r l}\left(x_{l}^{*}\right) d x_{l}^{*} \approx \frac{x_{l}}{2}\left[q_{r l}(0)+q_{r l}\left(x_{l}\right)\right]
$$

Evaluate temperature distribution within the single layer $S_{1}$ :

$$
T_{s}\left(x_{s}\right)=T_{s}(0)-\frac{Q_{t o t}}{k_{s}} x_{s}+\frac{1}{k_{s}} \int^{x_{s}} q_{r s}\left(x_{s}^{*}\right) d x_{s}^{*}
$$

where

$$
\int^{x_{j}} q_{r j}\left(x_{j}^{*}\right) d x_{j}^{*} \approx \sum_{p=1}^{N} \frac{\Delta x_{s}}{2}\left[q_{r s}\left(x_{s p}\right)+q_{r s}\left(x_{s(p+1)}\right)\right]
$$

And $\Delta x_{s}=D_{s} / N, \mathrm{~N}$ is integer.

The Maple software and numerical methods such as iteration were used in calculating the solutions. Software codes were written specifically for the calculation of temperature distribution and heat flux. 


\section{Reference}

1. Schulz, U., Leyens, C., Fritscher, K., Peters, M., Saruhan-Brings, B., Lavigne, O., Dorvaux, J-M., Poulain, M., Mevrel, R. and Caliez, M. "Some recent trends in research and technology of advanced thermal barrier coatings", Aerospace Science and Technology, 7 (2003) p.73-80.

2. Jones, R. L., "Thermal barrier coatings", Metallurgical and Ceramic Protective Coatings, Edited by Kurt H. Stern, Published in 1996 by Chapman \& Hall, London. ISBN 0412544407.

3. Morrell, P. and Rickerby, D. S., “Advantages/disadvantages of various TBC systems as perceived by the engine manufacturer", AGARD-R0823, (1998) paper 20.

4. Driver, D., Hall, D. W. and Meetham, G. W., in "The development of the gas turbine engine", Applied Science Publishers (London), 1981.

5. Nicholls, J. R., Lawson, K. J., "Methods to reduce the thermal conductivity of EBPVD TBCs", Surface and Coating Technology, 151-152 (2002), p.383-391.

6. Siegel, R. and Spuckler, C. M., "Analysis of thermal radiation effects on temperatures in turbine engine thermal barrier coatings", Materials Science and Engineering, A245 (1998), p.150-159.

7. Clarke, D. R. and Levi, C. G., "Materials design for the next generation thermal barrier coatings", Annual Review of Materials Research, 33 (2003), p.383-417.

8. Nicoll, A.R. and Wahl, G., "The effect of alloying additions on M-Cr-Al-Y systems An experimental study", Thin Solid Films, 95(1992), p.21-34.

9. Tawancy, H. M., Abbas, N. M. and Bennett, A., "Role of Y during high temperature 249 
oxidation of an M-Cr-Al-Y coating on an Ni-base superalloy", Surface and Coatings Technology, 68/69 (1994) p.10-16.

10. Stott, F. H. and Wood, G. C., "Growth and adhesion of oxide scales on $\mathrm{Al}_{2} \mathrm{O}_{3}-$ forming alloys and coatings", Materials Science and Engineering, 87 (1987), p.267274.

11. Stecura, S. "Effects of yttrium, aluminum and chromium concentrations in bond coatings on the performance of zirconia-yttria thermal barriers", Thin Solid Films, $73(1980)$, p.481-489.

12. Stecura, S. "Two-layer thermal barrier coatings 1: Effects of composition and temperature on oxidation behavior and failure", Thin Solid Films, 182(1989), p.121139.

13. Meier, S.M. and Gupta, D. K., "The evolution of thermal barrier coatings in gas turbine applications", Transactions of the ASME, Vol. 116(1994), p.250-257.

14. Bose, S., DeMasi-Marcin, J., "Thermal barrier coating experience in the gas turbine engine at Pratt \& Whitney", in NASA CP3312 (1995), p.63-78.

15. Schulz, U., Fritscher, K., Leyens, C., Peters, M. and Kaysser, W. A., "The thermocyclic behavior of differently stabilized and structured EB-PVD TBCs", The Journal of the Minerals, Metals \& Materials, Vol. 49. No. 10(1997), p.1-10.

16. Cawley, J. D., "Overview of zirconia with respect to gas turbine applications", NASA Technical Paper, 2286 (1984).

17. Sakuma, T., "Microstructural aspects on the cubic-tetragonal transformation in zirconia", Key Engineering Materials Vol. 153-154 (1998) p.75-96.

18. Howard, C. J., Hill, R. J. and Reichert, B. E., "Structure of the $\mathrm{ZrO}_{2}$ polymorphs at 250 
room temperature by high-resolution neutron powder diffraction", Acta Crystallographica B44 (1988), p.116-120.

19. Bocanegra-Bernal, M. H., "Phase transitions in zirconium dioxide and related materials for high performance engineering ceramics". Journal of Materials Science 37 (2002) p.4947-4971.

20. Kisi, E. H. and Howard, C. J. "Crystal structures of zirconia phases and their interrelation", Key Engineering Materials, Vols. 153-154 (1998), p.1-36.

21. Kuwabara, A., Katamura, J., Ikuhara, Y., and Sakuma, T., "Influence of interaction between neighboring oxygen ions on phase stability in cubic zirconia", Journal of the American Ceramic Society, 85(2002), p.2557-2561.

22. Kountouros, P. and Petzow, G., "Defect chemistry, phase stability and properties of zirconia polycrystals", in Science and Technology of. Zirconia V (Eds: SPS Badwal, MJ Bannister, RHJ Hannink), Technomic, Lancaster, Basel, (1993), p.30-48.

23. Aleksandrov, V. I., Osiko, V. V., Prokhorov, A. M. and Tatarintsev, V. M., "Synthesis and crystal growth of refractory materials by RF melting in a cold container", Current Topics in Materials Science, North-Holland Pub. Co.; Elsevier North-Holland, 1(1978), p.421-480.

24. Li, P., Chen, I. W., and Penner-Hahn, J. E., "X-ray-absorption studies of zirconia polymorphs. I. Characteristic local structures", Physical Review B, 48(1993-II), p.10063-10073.

25. Scott, H. G., "Phase relationships in the zirconia - yttria system", Journal of Materials Science, 10(1975), p.1527-1535.

26. Ingel, R. P. and Lewis III, D., "Lattice parameters and density for $\mathrm{Y}_{2} \mathrm{O}_{3}$-stabilized 251 
ZrO2", Journal of the American Ceramic Society, 69 [4] (1986), p.325-332.

27. Taraya, H., "Effect of $\mathrm{YO}_{1.5}$ dopant on unit-cell parameters of $\mathrm{ZrO}_{2}$ at low contents of $\mathrm{YO}_{1.5}$ ", Journal of the American Ceramic Society, 72 [4](1989), p.662-664.

28. Virkar, A. V., and Matsumoto, R. L. K., "Ferroelastic domain switching as a toughening mechanism in tetragonal zirconia", Journal of the American Ceramic Society, 69(1986), p.224-226.

29. Carlas G. Levi, "Emerging materials and processes for thermal barrier systems", Current Opinion in Solid State and Materials Science 8 (2004) p.77-91.

30. Leclercq, B. and Mevrel, R., "Thermal conductivity of zirconia-based ceramics for thermal barrier coatings", in Proc. CIMTEC (2002), Firenze, Italy.

31. S. Alperine, V. Arnault, O. Lavigne, R. Mevrel, "Heat barrier composition, a mechanical superalloy article provided with a ceramic coating having such a composition, and a method of making the ceramic coating", US Patent (2001), US $6,333,118 \mathrm{~B} 1$.

32. Stecura, S., "Effects of compositional changes on the performance of a thermal barrier coating system", NASA TM 78976(1979).

33. Stecura, S., "Optimization of the NiCrAl-Y/ZrO2-Y2O3 thermal barrier system", NASA Tech. Memo, 86905(1985).

34. Dwivedi, A. and Cormack, A. N., “A. Computer Simulation Study of the. Defect Structure of Calcia-Stabilised Zirconia", Philosophical Magazine A. 61(1990), p.122.

35. Cao, X.Q., Vassen, R., Stoever, D., "Ceramic materials for thermal barrier coatings", Journal of the European Ceramic Society 24(2004) p.1-10. 
36. Beele, W., Marijnissen, G. and Lieshout, A. V., "The evolution of thermal barrier coatings - status and upcoming solutions for today's key issues", Surface and Coatings Technology, 120-121(1999), p.61-67.

37. Singh, J. and Wolfe, D. E., "Review: Nano and macro-structured component fabrication by electron beam-physical vapor deposition (EB-PVD)", Journal of Materials Science, 40(2005), p.1-26.

38. Singh, J., and Wolfe, D. E., "Electron beam-physical vapor deposition technology: present and future applications", IMAST Quarterly, 3(2000), p.3-6.

39. Kaysser, W. A., Peters, M., Fritscher, K., and Schulz, U. (1998), "Processing, characterization and testing of EB-PVD thermal barrier coatings", in 85th Meeting of the AGARD Structures and Material Panel, Vol. AGARD report 823 at Aalborg, Denmark; AGARD, Neuilly Sur Seine, France, paper 9.

40. Gell, M., Xie, L., Jordan, E. H., and Padture, N. P., "Mechanisms of spallation of solution precusor plasma spray thermal barrier coatings", Surface \& Coating Technology, 188-189(2004), p.101-106.

41. Morrell, P. and Rickerby, D. S., “Advantages/disadvantages of various TBC systems as perceived by the engine manufacturer", in $85^{\text {th }}$ Meeting of AGARD Structures and Materials Panel, AGARD report 823 at Denmark; 1998.

42. Nicholls, J. R., Jaslier, Y., and Rickerby, D. S., "Erosion and foreign object damage of thermal barrier coatings", Material Science Forum, 251-254(1997), p.935-948.

43. Wellman, R.G., Deakin, M. J., Nicholls, J. R., “The effect of TBC morphology on the erosion rate of EB PVD TBCs", Wear, 258(2005), p.349-356.

44. Toriz, F. C., Thakker, A. B., Gupta, S. K., "Flight service evaluation of thermal 253 
barrier coatings by physical vapour deposition at $5200 \mathrm{H}^{\prime \prime}$, Surface and Coating Technology, 39/40(1989), p.161-172.

45. Wolfe, D. E., Singh, J., Miller, R. A., Eldridge, J. I., and Zhu, D., "Tailored microstructure of EB-PVD 8YSZ thermal barrier coatings with low thermal conductivity and high thermal reflectivity for turbine applications", Surface and Coatings Technology, 19(2005), p.132-149.

46. Wolfe, D. E., Singh, J., "Synthesis and characterization of TiBCN coatings deposited by ion beam assisted, co-evaporation electron beam-physical vapor deposition (EBPVD)", Journal of Materials Science, 37(2002), p.3777-3787.

47. Wright, P. K. and Evans, A. G., "Mechanisms governing the performance of thermal barrier coatings", Current Opinion in Solid State and Materials 4(1999), p.255-265.

48. Zhu, D., Choi, S. R., and Miller, R., "Development and thermal fatigue testing of ceramic thermal barrier coatings", Surface \& Coatings Technology, 188-189(2004), p.146-152.

49. McDonald, G. and Hendricks, R. C., "Effect of thermal cycling on ZrO2-Y2O3 thermal barrier coatings", Thin Solid Films, 73(1980), p.491-496.

50. Zhao, X., Wang, X., and Xiao, P., "Sintering and failure behavior of EB-PVD thermal barrier coating for isothermal treatment", Surface \& Coatings Technology, 200 (2006), p.5946-5955.

51. Padture, N. P., Gell, M., Jordan, E. H., "Thermal barrier coatings for gas-turbine engine applications", Science, 296(2002), p.280-284.

52. Tolpygo, V. K., Clarke, D. R., and Murphy, K. S., "Oxidation-induced failure of EBPVD thermal barrier coatings", Surface \& Coatings Technology, 146-147(2001), 
p.124-131.

53. Tolpygo, V. K. and Clarke, D. R., "Wrinkling of $\alpha$-Alumina films grown by oxidation - II. Oxide separation and failure", Acta Materialia, 46(1998), p.5167-5174.

54. Alperine, S., Derrien, M., "Thermal barrier coatings: the thermal conductivity challenge", NATO Workshop on Thermal Barrier Coatings, Aalborg, Denmark, AGARD-R-823 (1998), p.1.

55. Goedien, J. G., Sabol, S., and Dighe, S., "Westinghouse thermal barrier coatings development", NETL Conference Proc. (1997), ATS5-10.

56. Rabiei and A. G. Evans, "Failure mechanisms associated with the thermally grown oxide in plasma sprayed thermal barrier coatings", Acta Materialia, 48(2000), p.39633976.

57. Mifune, N., Harada, Y., Doi, T., and Yamasaki, R., "Hot-corrosion behavior of graded thermal barrier coatings formed by plasma-spraying process", Journal of Thermal Spray Technology, 13(2004), p. 561-569.

58. Jones, R. L., Reidy, R. F., and Mess, D., "Scandia, yttria-stabilized zirconia for thermal barrier coatings", Surface \& Coatings Technology, 82(1996), p.70-76.

59. Thompson, J.A., Ji, W., and Clyne, T. W., "The effect of a high thermal gradient on sintering and stiffening in the top coat of a thermal barrier coating (TBC) system", in Thermal Spray 2003: Advancing the Science \& Applying the Technology (ed. C.Moreau, B.Marple), Proceedings of the 2003 International Thermal Spray Conference, 5-8 May 2003, Orlando, Florida, USA, Tsipas, S.A., Golosnoy I.O., and Clyne, T.W., ASM International (2003), p.1547-1552.

60. Fritscher, K., Szucs, F., Schulz, U., Saruhan, B., Peters, M., and Kaysser, W. A., 255 
"Impact of thermal exposure of EB-PVD TBCs on young's modulus and sintering", Ceramic Engineering and Science Proceedings, 23(2002), p.341-352.

61. Tsipas, S.A., Golosnoy, I.O., Damani, R., and Clyne, T.W., "The effect of a high thermal gradient on sintering and stiffening in the top coat of a thermal barrier coating system", Journal of Thermal Spray Technology, 13(2004), p.370-376.

62. Zhao, X., Wang, X., and Xiao, P., "Sintering and failure behavior of EB-PVD thermal barrier coating after isothermal treatment”, Surface \& Coatings Technology, vol. 200, issue 20-21(2006), p. 5946-5955.

63. Lughi, V. and Clarke, D. R., "High temperature aging of YSZ coatings and subsequent transformation at low temperature", Surface \& Coatings Technology, 200(2005), p. 1287-1291.

64. Schulz, U., "Phase transformation in EB-PVD yttria partially stabilized zirconia thermal barrier coatings during annealing", Journal of the American Ceramic Society, 83(2000), p.904-910.

65. Li, M. H., Sun, X. F., Gong, S. K., Zhang, Z. Y., Guan, H. R. and Hu, Z. Q., "Phase transformation and bond coat oxidation behavior of EB-PVD thermal barrier coating”, Surface \& Coating Technology, 176(2004), p. 209-214.

66. He, M. Y., Mumm, D. R. and Evans, A.G., "Criteria for the delamination of thermal barrier coatings: with application to thermal gradients", Surface \& Coatings Technology, 185(2004), p.184-193.

67. Noma, T., Yoshimura, M., Somiya, S., "Stability of diffusionlessly transformed tetragonal phases in rapidly quenched $\mathrm{ZrO}_{2}-\mathrm{Y}_{2} \mathrm{O}_{3}$ ", in Advances in Ceramics, Vol. 24(1988): Science and Technology of Zirconia III, eds., S. Somiya, N.Yamamoto and 256 
H. Yanagida, The American Ceramic Society, Westerville OH., p.377-384.

68. Kittel. C., Introduction to solid state physics. $5^{\text {th }}$ Ed., John Wiley \& Sons, Inc. 1976.

69. Berman, R., Thermal conduction in solids, Clarendon Press, Oxford, UK, 1976.

70. Srivastava, G. P., The physics of phonons, Adam Hilger, Bristol, 1990.

71. Klemens, P. G., "Thermal conductivity and lattice vibrational modes", Solid State Physics, Eds. F. Seitz and D. Turnbull. Academic Press, New York, 7(1958), p.1-98.

72. Klemens, P.G., "Theory of the Thermal Conductivity of Solids", Thermal conductivity, edited by R. P. Tyne (Academic Press, London, UK, 1(1969), p.1-68.

73. Roufosse, M. and Klemens, P. G., "Thermal conductivity of complex dielectric crystal", Physical Review B, 7(1973), p.5379-5386.

74. Klemens, P. G., "Thermal conductivity of zirconia", Thermal Conductivity, edited by K.E.Wilkes et al., Technomics, Lancaster, PA, 23 (1996), p.209-220.

75. Klemens, P. G., "Phonon scattering by oxygen vacancies in ceramics", Physica B, 263-264 (1999), p.102- 104.

76. Callaway, J., "Model for lattice thermal conductivity at low temperatures", Physical Review, 113(1959), p.1046-1051.

77. Callaway, J. and Baeyer, H. C., "Effects of point imperfections on lattice thermal conductivity", Physical Review, 120(1960), p.1149-1154.

78. Eldridge, J. I., Spuckler, C. M., and Street, K.W., "Infrared radiative properties of yttria-stabilized zirconia thermal barrier coatings", $26^{\text {th }}$ Annual Conference of Composites, Advanced Ceramics, Materials and Structures: B, Cocoa Beach, FL, Westerville, $\mathrm{OH}$, American Ceramic Society, 13-18(2002), p.417-430.

79. Wahiduzzaman, S. and Morel, T., "Effect of translucence of engineering ceramics on 257 
heat transfer in diesel engines", NASA report, ORNL/Sub/86-22042/1.(1987).

80. Zhu, D., Bansal, N. P., Miller, R. A., "Thermal conductivity and stability of $\mathrm{HfO}_{2}$ $\mathrm{Y}_{2} \mathrm{O}_{3}$ and $\mathrm{La}_{2} \mathrm{Zr}_{2} \mathrm{O}_{7}$ evaluated for $1650^{\circ} \mathrm{C}$ thermal/environmental barrier coating applications", Ceramic Transactions, 153(2004), p.331-343.

81. Siegel, R., Spuckler, C. M., "Analysis of thermal radiation effects on temperatures in turbine engine thermal barrier coatings", Materials Science and Engineering, A245 (1998), p.150-159.

82. Siegel, R. and Howell, J. R., Thermal radiation heat transfer, Taylor \& Francis; 4th edition, 2001.

83. Sodeoka, S., Suzuki, M, Inoue, T. and Ueno, K, “Thermal and mechanical properties of plasma sprayed coatings", in Thermal Spray: Practical Solutions for Engineering Problems. Ed. C. C. Berndt, ASM, Metals Park, OH, (1996), p.295-301.

84. Rickerby, D. S., "A metallic article having a thermal barrier coating and a method of application thereof', European Patent EP 0825271 A1, (1998).

85. Tamarin, Y. A., Kachanov, E. B., Zherzdev, S.V., "Thermophysical properties of ceramic layers in EB-TBC", $4^{\text {th }}$ Inst. Symposium, High Temperature Corrosion (Les Embiez, France, May 1996), Material Science, Forum 251-254 (1997), p.949-956.

86. Raghavan, S., Wang, H., Porter, W. D., Dinwiddie, R. B., and Mayo, M. J., "Thermal properties of zirconia co-doped with trivalent and pentavalent oxides", Acta Materialia, 49 (2000), p.169-179.

87. Zhu, D., Chen, Y. L., Miller, R. A., "Defect clustering and nano-phase structure characterization of multi-component rare earth oxide doped zirconia -yttria thermal barrier coatings", American Ceramic Society, 23(2002), p.457-468. 
88. Zhu D. and Miller R. A., "Thermal conductivity and sintering behaviour of advanced thermal barrier coatings", Ceramic Engineering and Science Proceedings, 23(2002), p.457-468.

89. Zhu D., Nesbitt J. A., et al., "Furnace cyclic behaviour of plasma sprayed zirconia yttria and multi-component rare earth oxide doped thermal barrier coatings", Ceramic Engineering and Science Proceedings, 23(2002), p.533-545.

90. Hass, D. D., Slifka A. J. and Wadley, H. N. G., "Low thermal conductivity vapor deposited zirconia microstructures", Acta Materialia, 49 (2001), p.973-983.

91. Gu, S., Lu, T. J., Hass, D. D. and Wadley, H. N. G., "Thermal conductivity of zirconia coatings with zig-zag pore microstructures", Acta Materialia, 49 (2001), p.2539-2547.

92. Brandon, J. R. and Taylor, R., "Phase stability of zironia-based thermal barrier coatings, Part I. Zirconia-yttria alloys", Surface \& Coatings Technology, 46 (1991), p.75-90.

93. Stanek, C. R., Minervini, L. and Grimes, R. W., "Nonstoichiometry in $\mathrm{A}_{2} \mathrm{~B}_{2} \mathrm{O}_{7}$ pyrochlores", Journal of the American Ceramic Society, 85(2002), p.2792-2798.

94. Minervini, L., Grimes, R. W., "Disorder in pyrochlore oxides", Journal of the American Ceramic Society, 83(2000), p.1873-1878.

95. Lehmann, H., Pitzer, D., Pracht, G., Vassen, R. and Stőver, D., "Thermal conductivity and thermal expansion coefficients of the lanthanum rare-earth-element zirconate system", Journal of the American Ceramic Society, 86(2003), p.1338-1344.

96. Maloney, M. J., "Thermal barrier coating systems and materials", US Patent $6,231,991 \mathrm{~B} 1,(2001)$. 
97. Stőver, D., Pracht, G., Lehmann, H., Dietrich, M., Dőring, J-E. and Vaßen, R., "New material concepts for the next generation of plasma-sprayed thermal barrier coatings", Journal of Thermal Spray Technology, 13 (2004), p.76-83.

98. Catcher G. L. and Rearick T. M., "O-anion transport measured in several $\mathrm{R}_{2} \mathrm{M}_{2} \mathrm{O}_{7}$ pyrochlores using perturbed - angular correction spectroscopy", Physical Review B, B52 (1995), p.9890-9899.

99. Van Dijk M. P., Vries K. J. D. and Burggroaf A. J., "Oxygen ion and mixed conductivity in compounds with the fluorite and pyrochlore structure", Solid State Ionics, 9-10(1983), p.913-20.

100. Mori, M., Abe, T., Itoh, H., Yamamoto, O., Shen, G. Q., Takeda, Y. and Imanishi, N., "Reaction mechanism between lanthanum manganite and yttria doped cubic zirconia", Solid State Ionics, 123(1999), p.113-119.

101. Vassen R., Cao X., Tieta F., Basu D., and Stőver D., "Zirconates as new materials for thermal barrier coatings", Journal of the American Ceramic Society, 83(2000), p.2023-2028.

102. Zhu, D., Bansal, N. P., and Miller, R. A., "Thermal conductivity and stability of $\mathrm{HfO} 2-\mathrm{Y} 2 \mathrm{O} 3$ and $\mathrm{La} 2 \mathrm{Zr} 2 \mathrm{O} 7$ evaluated for $1650 \mathrm{C}$ thermal/environmental barrier coating application", Ceramic Transactions, 153(2004), p.331-343.

103. Cao XQ, Vassen R., Stőver D., "Ceramic materials for thermal barrier coatings", Journal of the European Ceramic Society, 24(2004), p.1-10.

104. Nicholls, J. R., Lawson, K. J., "Low thermal conductivity EB-PVD thermal barrier coatings", Material Science Forum, 369 - 372(2001), p. 595-606.

105. Allen, W. P., "Reflective coatings to reduce radiation heat transfer", US patent 260 
US $0008170 \mathrm{~A} 1,(2003)$.

106. Wang, D., Huang, X., Patnaik, P.C., "Design of a multilayered thermal barrier coating structure for optimized thermal properties", 43 Annual Conference of Metallurgists of CIM, Hamilton, Ontario/Canada, (2004), p.93-107.

107. Wang, D., Huang, X., Patnaik, P.C., "Design and modeling of multiple layered TBC system with high reflectance", Journal of Material Science, Accepted for publication, (2006), in press.

108. Reed, J. S., "Principles of ceramic processing", $2^{\text {nd }}$ Ed., Wiley-Interscience, New York, 1995.

109. Barsoum, M., "Foundamentals of ceramics", McGraw-Hill, New York, 1997, Chapt. 10.

110. Gibson, I. R., "Sinterability of commercial $8 \mathrm{~mol} \%$ yttria-stabilized zirconia powders and the effect of sintered density on the ionic conductivity", Journal of Materials Science, 33(1998), p. 4297-4305.

111. Callister, W. D., "Materials science and engineering: An introduction", $6^{\text {th }}$ edition, John Wiley \& Sons, Inc., 2003, p.91-106.

112. Chiang, Y. M., Birnie, D., and Kingery, W. D., "Physical ceramics: principles for ceramic science and engineering", John Wiley \& Sons, Inc., 1997, Fig. 3-1.

113. Rahaman, M. N., "Ceramic processing and sintering", 2nd ed., Revised and Extended, Marcel Dekker, New York, 2003, p.73.

114. "Standard test methods for apparent porosity, water absorption, apparent specific gravity, and bulk density of burned refractory brick and shapes by boiling water", C20-00 (2001), Annual Book of ASTM Standards, 15.01. American Society for $261^{\circ}$ 
Testing and Materials, West Conshohocken, PA. p. 6-8.

115. Miller, R. A., Smialek, J. L. and Garlick, R. G., "Phase stability in plasmasprayed, partially stabilized zirconia-yttria", in Advances in Ceramics, Science and Technology of Zirconia I, Edited by A. H. Heuer and L. W. Hobbs. American Ceramic Society, Columbus, $\mathrm{OH}, 3(1981)$, p.241-253.

116. JEOL LTD, "Invitation to the SEM World", SEM Document from JEOL.

117. Ladd, M. F. C. and Palmer, R. A., "Structure determination by x-ray crystallography", Plenum Press, New York and London, 1994.

118. Brandon, J. R., and Taylor, R., "Phase stability of zirconia-based thermal barrier coatings, Part I. Zirconia-yttria alloys", Surface \& Coatings Technology, 46(1991), p.75-90.

119. James Day, M. A. Sci., Thesis, Carleton University, 2004.

120. Kogut, L. and Komvopoulos, K., "Determination of real material properties from nanoindentation experiments", Proceedings of 2003 STLE/ASME Joint International Tribology Conference Ponte Vedra Beach, Florida USA, October 26-29(2003), p.1-3.

121. Mackenzie, J. K., "The elastic constants of a solid containing spherical holes", Proceedings of the Physical Society, London, 63B(1950), p.2-11.

122. Houlbert, A. S., Cielo, P., Moreau, C., and Lamontagne, M., "Measurement of thermal diffusivity and anisotropy of plasma-sprayed coatings", International Journal of Thermophysics, 15(1994), p.525-546.

123. Howard, C. J., "Structures of the $\mathrm{ZrO} 2$ polymorphs at room temperature by highresolution neutron powder diffraction", Acta Crystallographica, B44 (1988), p.116120. 
124. Theunissen, G. S. A. M., Winnubst, A. J. A., and Burggraaf, A. J. "Effect of dopants on the sintering behavior and stability of tetragonal zirconia ceramics", Journal of the European Ceramic Society, 9(1992), p.251-263.

125. Leclercq, B. and Mevrel, R., "Thermal conductivity of zirconia-based ceramics for thermal barrier coatings", in Proceedings, CIMTEC 2002, Firenze, Italy, (2002), p. $365-372$.

126. Tojo, T. and Atake, T., "Heat capacity of yttria- and scandia-doped zirconia", Solid State Ionics: New Developments, Asian Conference on Solid State Ionics, edited by B. V. R. Chowdari et al., Kandy, Sri Lanka, (1996), p.529-533

127. Klemens, P. G., "Thermal conductivity of inhomogeneous media", HighTemperature, High-Pressure, 23 (1991), p.241-248.

128. Leclercq, B., Mevrel, R., Liedtke, V. and Hohenauer, W., "Thermal conductivity of zirconia-based ceramics for thermal barrier coating", Mat-wiss. U. Werkstofftech, 34(2003), p.406-409.

129. Mevrel, R., Laizet, J-C., Azzopardi, A., Leclercq, B., Poulain, M., Lavigne, O. and Demange, D., "Thermal diffusivity and conductivity of $\mathrm{Zr}_{1-\mathrm{x}} \mathrm{Y}_{\mathrm{x}} \mathrm{O}_{2-\mathrm{x} / 2}(\mathrm{x}=0,0.084$ and 0.179) single crystal", Journal of European Ceramic Society, 24(2004), p.30813089.

130. Fukushima, S., Ohmichi, T. and Handa, M., "The effect of rare earths on thermal conductivity of uranium, plutonium and their mixed oxide fuels", Journal of the Less Common Metals, 121(1986), p.631-636.

131. Zacate, M. O., Minervini, L., Bradfield, D. J., Grimes, R. W. and Sickafus, K. E., "Defect cluster formation in $\mathrm{M}_{2} \mathrm{O}_{3}$-doped cubic $\mathrm{ZrO}_{2}$ ", Solid State Ionics, 263 
$128(2000)$, p.243-254.

132. Clarke, D. R., "Material selection guidelines for low thermal conductivity thermal barrier coatings", Surface and Coatings Technology, 163-164(2003), p.67-74.

133. Fröger, F. A., "The chemistry of imperfect crystals", North Holland Publishing Co., Amsterdam, 1964.

134. Macleod, H. A., "Thin-film optical filters", $2^{\text {nd }}$ ed., Published by Adam Hiller Ltd. (1986), p.158-186.

135. Ditchburn, R.W. "Light”, 2nd ed., London: Blackie \& Sons, (1963), p.582-585.

136. Handbook of Optical Constants of Solids, ED Palik ed., Academic Press, New York (1985).

137. Wood D. L. and Nassau K., "Refractive index of cubic zirconia stabilized with yttria”, Applied Optics, 21(1982), p.2978-2981.

138. Wood D.L., Nassau K., and Kometani T.Y., "Refractive index of $\mathrm{Y}_{2} \mathrm{O}_{3}$ stabilized cubic zirconia: variation with composition and wavelength", Applied Optics, 29(1990), p.2485-2488.

139. Eriksson T.S., Hjortsberg A., Niklasson G.A., and Granquist C. G., "Infrared optical properties of evaporated aluminia films", Applied Optics, 20(1981), p.27422746.

140. Jerman M., Qiao Z., and Merger D., "Refractive index of thin films of SiO2, $\mathrm{ZrO} 2$, and $\mathrm{HfO} 2$ as a function of the films' mass density", Applied Optics, 44(2005), p.3006-3012.

141. Manara, J., Brandt, R, Kuhn, J., and Fricke, J., "Emittance of Y2O3 stabilized $\mathrm{ZrO} 2$ thermal barrier coatings prepared by electron-beam physical-vapor deposition", 
High Temperature - High Pressure, Vol. 32, 2000, p.361-368.

142. Connolly, J. R., "Introduction to x-ray powder diffraction", for EPS400-002, Spring 2005.

143. Yashima, M., Sasaki, S., Kakihana, M., Yamaguchi, Y., Arashi, H., Yoshimura, M., "Oxygen-induced structural change of the tetragonal phase around the tetragonalcubic phase boundary in $\mathrm{ZrO}_{2}-\mathrm{YO}_{1-5}$ solid solutions". Acta Crystallographica, B50, 663 (1994).

144. Negita, K. and Takao, H., "Condensations of phonons at the tetragonal to monoclinic phase transition in ZrO2", Journal of Physics and Chemistry of Solids 50 (3) (1989), p.325-331.

145. Youngblood, G. E., Rice, R. W., Ingel, R. P., "Thermal diffusivity of partially and fully stabilized (yttria) zirconia single crystals", Journal of the American Ceramic Society, 71(4)(1988), p.255-260.

146. Slack, G. A., "The thermal conductivity of non-metallic crystals", Solid State Physics, 34(1979), p.1-71.

147. Wang, D., Huang, X., Patnaik, P.C., "Latest advancements in thermal barrier coatings", Canadian Aeronautics and Space Journal, 50(2004) p.107-114.

148. Rebollo N. R., Fabrichanayo O., Levi C. G., "Phase stability of Y+Gd co-doped zirconia", Zeit Metal, 94(2003), p.164-70.

149. Rebollo N. R., Grandhi A. S., Levi C. G., "Phase stability issues in emerging TBC systems", In: Opila E. J., Hou P., et al., editors. High Temperature Corrosion and Materials Chemistry IV, Electro-chemical Society Proceedings, PV-2003-16 (2003), p.431-42. 
150. Wu J., Wen X., Padture N. P., Klemens P. G., Gell M., Garcia E., Miranzo P., Osendi M. I., "Low thermal conductivity rare earth zirconates for potential thermal barrier coating applications", Journal of the American Ceramic Society, 85(2002), p.3031-3035.

151. Ambegaokar, V., "Thermal resistance due to isotopes at high temperatures", Physical Review, 114(1959), p.488 - 489.

152. Abeles, B., "Lattice thermal conductivity of disordered semiconductor alloys at high temperatures", Physical Review, 131(1963), p.1906-1911.

153. Fukushima, S., Ohmichi, T., and Handa, M., "The effect of rare earths on thermal conductivity of uranium, plutonium and their mixed oxide fuels", Journal of LessCommon Metals, 121(1986), p.631 - 636.

154. Clarke, D. R., "Materials selection guidelines for low thermal conductivity thermal barrier coatings", Surface \& Coatings Technology, $163-164(2003)$, p.67-74.

155. Raghaven, S., Wang, H., Dinwiddie, R. B., Porter, W. D., and Mayo, M. J., "The effect of grain size, porosity and yttria content on the thermal conductivity of nanocrystalline zirconia", Scripta Materialia, 39(8)(1998), p.1119-1125. 